\title{
RERTR-12 Insertion 2 Irradiation Summary Report
}

D. M. Perez

G. S. Chang

D. M. Wachs

G. A. Roth

N. E. Woolstenhulme

September 2012

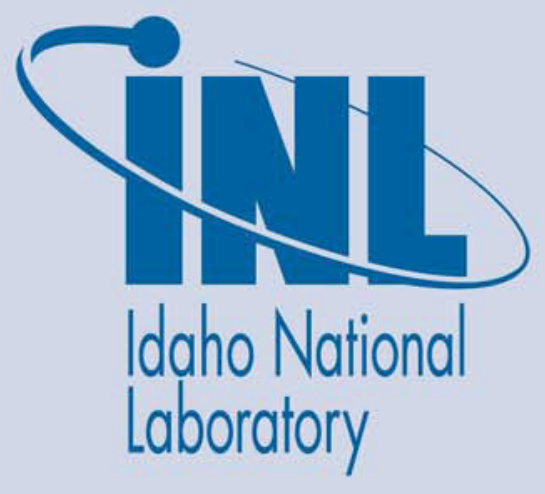

The INL is a U.S. Department of Energy National Laboratory operated by Battelle Energy Alliance 


\section{DISCLAIMER}

This information was prepared as an account of work sponsored by an agency of the U.S. Government. Neither the U.S. Government nor any agency thereof, nor any of their employees, makes any warranty, expressed or implied, or assumes any legal liability or responsibility for the accuracy, completeness, or usefulness, of any information, apparatus, product, or process disclosed, or represents that its use would not infringe privately owned rights. References herein to any specific commercial product, process, or service by trade name, trade mark, manufacturer, or otherwise, does not necessarily constitute or imply its endorsement, recommendation, or favoring by the U.S. Government or any agency thereof. The views and opinions of authors expressed herein do not necessarily state or reflect those of the U.S. Government or any agency thereof. 


\title{
RERTR-12 Insertion 2 Irradiation Summary Report
}

D. M. Perez, G. S. Chang, D. M. Wachs,

\author{
G. A. Roth, and N. E. Woolstenhulme
}

September 2012

Idaho National Laboratory Idaho Falls, Idaho 83415

http://www.inl.gov

Prepared for the

U.S. Department of Energy

Office of National Nuclear Security

Administration

Under DOE Idaho Operations Office

Contract DE-AC07-05ID14517 


\title{
RERTR-12 Insertion 2 Irradiation Summary Report
}

\author{
INL/EXT-12-27085
}

Revision 0

September 2012

Approved by:

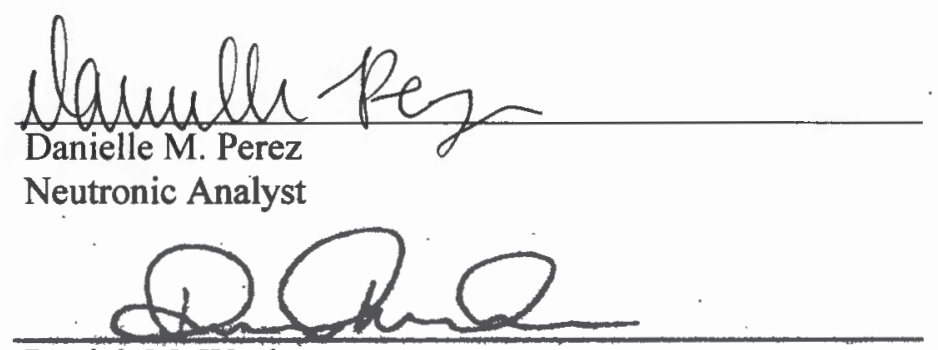

Daniel. M. Wachs

Principal Investigator

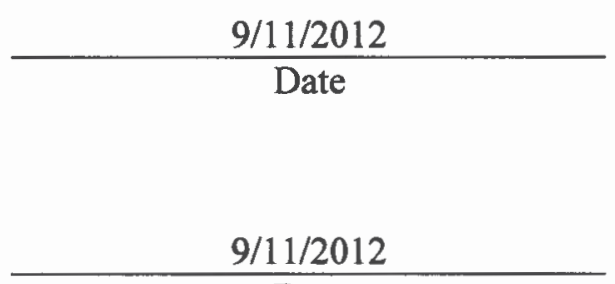

Date

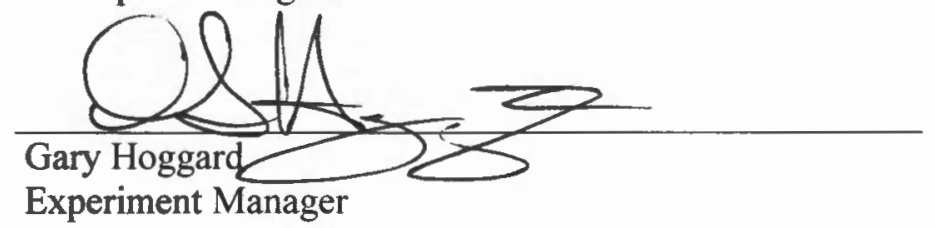

$9 / 11 / 2012$

Date 


\section{SUMMARY}

The Reduced Enrichment for Research and Test Reactor (RERTR) experiment RERTR-12 was designed to provide comprehensive information on the performance of uranium-molybdenum (U-Mo) based monolithic fuels for research reactor applications. ${ }^{1}$ RERTR-12 insertion 2 includes the capsules irradiated during the last three irradiation cycles. These capsules include Z, Y1, Y2 and Y3 type capsules.

The following report summarizes the life of the RERTR-12 insertion 2 experiment through end of irradiation, including as-run neutronic analysis results, thermal analysis results and hydraulic testing results. 


\section{CONTENTS}

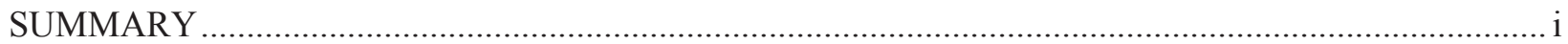

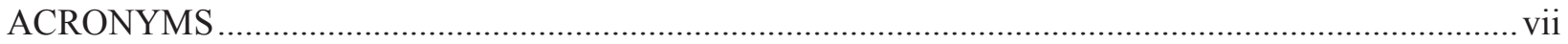

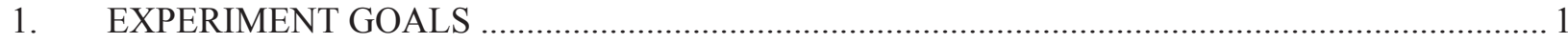

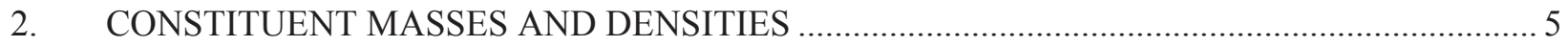

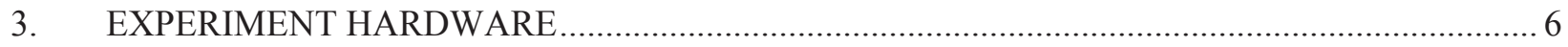

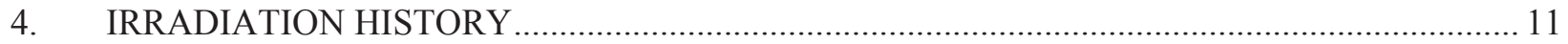

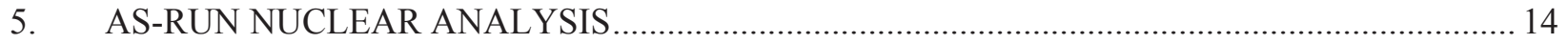

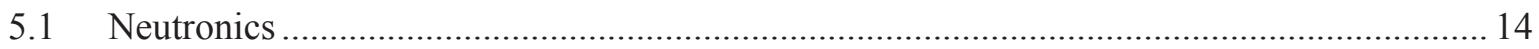

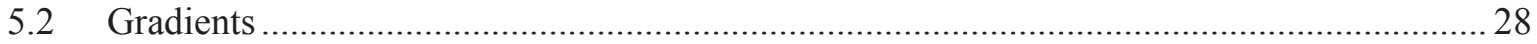

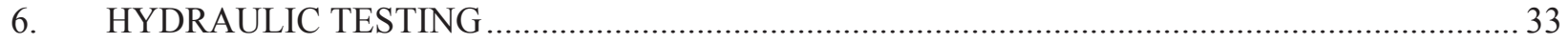

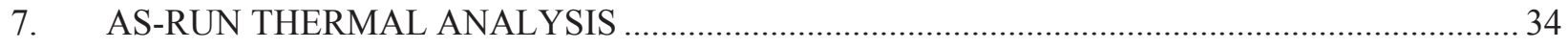

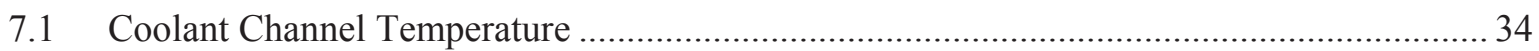

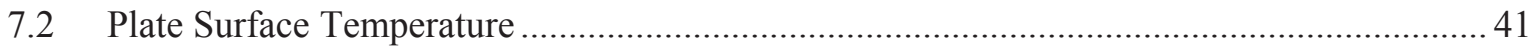

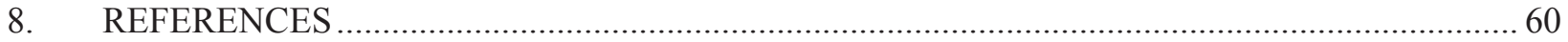

Appendix A Individual Plate Power and Fission Density Plots ........................................................... 61

Appendix B Fission Rate Local to Average Ratio 2D Gradient Maps .................................................. 79 


\section{FIGURES}

Figure 1: MCNP-Generated radial cross-section view of RERTR-12 test assembly (mini-plates C1 through $\mathrm{C} 4)$. 1

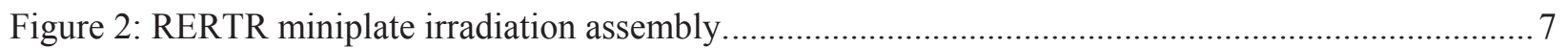

Figure 3: DWG-630244: RERTR monolithic fuel miniplate................................................................. 8

Figure 4: DWG-630245: RERTR thick (0.020 in) monolithic fuel miniplate.......................................... 9

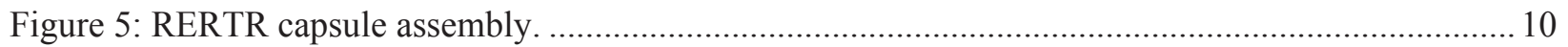

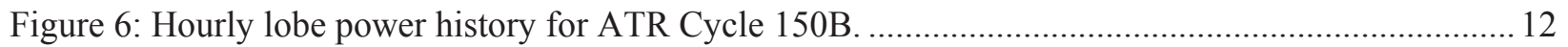

Figure 7: Hourly lobe power history for ATR Cycle 151A.................................................................. 12

Figure 8. Hourly lobe power history for ATR Cycle 151B................................................................. 13

Figure 9: BOL fission rate local to average ratios in the transverse direction for a type $\mathrm{Z}$ capsule

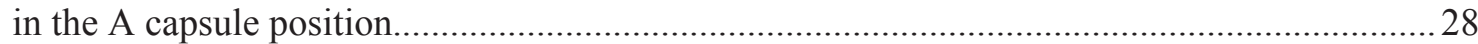

Figure 10: BOL fission rate local to average ratios in the transverse direction for a type $\mathrm{Y}$ capsule

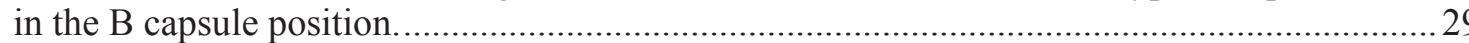

Figure 11: BOL fission rate local to average ratios in the transverse direction for a type $\mathrm{Y}$ capsule

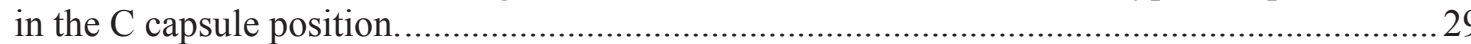

Figure 12: BOL fission rate local to average ratios in the transverse direction for a type $\mathrm{Y}$ capsule

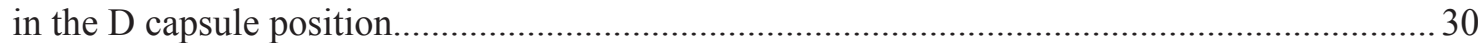

Figure 13: BOL fission rate local to average ratios in the axial direction for a type $\mathrm{Z}$ capsule in the A capsule position.

Figure 14: BOL fission rate local to average ratios in the axial direction for a type $\mathrm{Y}$ capsule in the $\mathrm{B}$ capsule position.

Figure 15: BOL fission rate local to average ratios in the axial direction for a type $\mathrm{Y}$ capsule in the $\mathrm{C}$ capsule position.

Figure 16: BOL fission rate local to average ratios in the axial direction for a type $\mathrm{Y}$ capsule in the $\mathrm{D}$ capsule position

Figure 17: RERTR-12 capsule cross section with the front (side with plate ID) of plate 1 facing the core.

Figure 18: Coolant channel temperatures as a function of location along the RERTR-12 test assembly at BOC $150 \mathrm{~B}(0.0 \mathrm{EFPD})$.

Figure 19: Coolant channel temperature as a function of location along the RERTR-12 test assembly at MOC1 150B (18.0 EFPD).

Figure 20: Coolant channel temperature as a function of location along the RERTR-12 test assembly at MOC2 150B (31.0 EFPD). 36

Figure 21: Coolant channel temperature as a function of location along the RERTR-12 test assembly at EOC 150B (41.9 EFPD). 36

Figure 22: Coolant channel temperatures as a function of location along the RERTR-12 test assembly at BOC 151A (0.0 EFPD).

Figure 23: Coolant channel temperature as a function of location along the RERTR-12 test assembly at MOC1 151A (15.0 EFPD). 
Figure 24: Coolant channel temperature as a function of location along the RERTR-12 test assembly at MOC2 151A (34.0 EFPD).

Figure 25: Coolant channel temperature as a function of location along the RERTR-12 test assembly at EOC 151A (56.1 EFPD) .38

Figure 26: Coolant channel temperature as a function of location along the RERTR-12 test assembly at BOC 151B (0.0 EFPD).

Figure 27: Coolant channel temperature as a function of location along the RERTR-12 test assembly at MOC1 151B (23.0 EFPD). 39

Figure 28: Coolant channel temperature as a function of location along the RERTR-12 test assembly at MOC2 151B (39.0 EFPD).

Figure 29: Coolant channel temperature as a function of location along the RERTR-12 test assembly at EOC 151B (51.3 EFPD). 40

\section{TABLES}

Table 1: RERTR-12 Insertion 2 Experiment Capsule Configuration 2

Table 2: RERTR-12-3 Experiment Matrix 2

Table 3: RERTR-12-4 Experiment Matrix …....................................................................................... 3

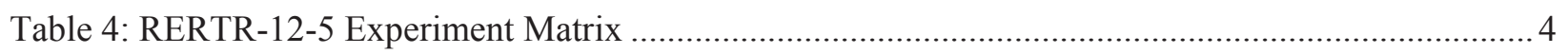

Table 5: RERTR-12 Constituent masses and densities for plates irradiated in the last three cycles ............ 5

Table 6: RERTR Irradiation Hardware Drawing List................................................................................ 6

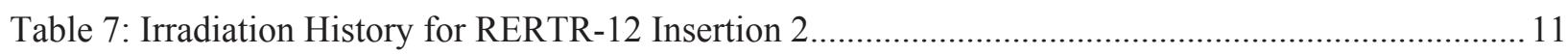

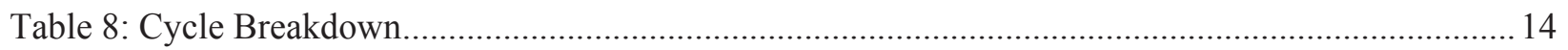

Table 9: RERTR-12 Calculated Nominal Initial Constituent Masses and Densities for Capsule Type Z Based off a Nominal Fuel Alloy Density of $17.2 \mathrm{~g} / \mathrm{cc}$ .14

Table 10: RERTR-12 Calculated Nominal Initial Constituent Masses and Densities for Capsule Type Y Based off a Nominal Fuel Alloy Density of $17.2 \mathrm{~g} / \mathrm{cc}$. 15

Table 11: MCNP-Calculated As-run Results for RERTR-12-3 Irradiated in ATR Position B-9 During Cycle 150B, BOC, Averaged North Lobe Power of $20.7 \mathrm{MW}^{4}$... 16

Table 12: MCNP-Calculated As-run Results for RERTR-12-3 Irradiated in ATR Position B-9 During Cycle 150B, MOC1 (18 EFPD), Averaged North Lobe Power of $20.7 \mathrm{MW}^{4}$ 17

Table 13: MCNP-Calculated As-run Results for RERTR-12-3 Irradiated in ATR Position B-9 During Cycle 150B, MOC2 (31 EFPD), Averaged North Lobe Power of $20.7 \mathrm{MW}^{4}$ 18

Table 14: MCNP-Calculated As-run Results for RERTR-12-3 Irradiated in ATR Position B-9 During Cycle 150B, EOC (41.9 EFPD), Averaged North Lobe Power of $20.7 \mathrm{MW}^{4}$..... 19

Table 15: MCNP-Calculated As-run Results for RERTR-12-4 Irradiated in ATR Position B-9 During Cycle 151A, BOC, Averaged North Lobe Power of $18.4 \mathrm{MW}^{5}$.... 20

Table 16: MCNP-Calculated As-run Results for RERTR-12-4 Irradiated in ATR Position B-9 During Cycle 151A, MOC1 (15 EFPD), Averaged North Lobe Power of $18.4 \mathrm{MW}^{5}$

Table 17: MCNP-Calculated As-run Results for RERTR-12-4 Irradiated in ATR Position B-9 During Cycle 151A, MOC2 (34 EFPD), Averaged North Lobe Power of $18.4 \mathrm{MW}^{5}$ 22 
Table 18: MCNP-Calculated As-run Results for RERTR-12-4 Irradiated in ATR Position B-9 During Cycle 151A, EOC (56.1 EFPD), Averaged North Lobe Power of $18.4 \mathrm{MW}^{5}$

Table 19: MCNP-Calculated As-run Results for RERTR-12-5 Irradiated in ATR Position B-11 During Cycle 151B, BOC, Averaged South Lobe Power of $22.7 \mathrm{MW}^{6}$

Table 20: MCNP-Calculated As-run Results for RERTR-12-5 Irradiated in ATR Position B-11 During Cycle 151B, MOC1 (18.0 EFPD), Averaged South Lobe Power of $22.7 \mathrm{MW}^{6}$ .25

Table 21: MCNP-Calculated As-run Results for RERTR-12-5 Irradiated in ATR Position B-11 During Cycle 151B, MOC2 (39.0 EFPD), Averaged South Lobe Power of $18.4 \mathrm{MW}^{6}$........... 26

Table 22: MCNP-Calculated As-run Results for RERTR-12-5 Irradiated in ATR Position B-11 During Cycle 151B, EOC (51.3 EFPD), Averaged South Lobe Power of $18.4 \mathrm{MW}^{6}$..... 27

Table 23: Loss Coefficients for the RERTR Irradiation Test Vehicle Components ${ }^{7}$ .33

Table 24: As-run minimum, maximum and average plate surface temperatures over fuel zone on the south side of the plate for plates irradiated in Cycle 150B, BOC (0 EFPD)

Table 25: As-run minimum, maximum and average plate surface temperatures over fuel zone on the north side of the plate for plates irradiated in Cycle 150B, BOC (0 EFPD).

Table 26: As-run minimum, maximum and average plate surface temperatures over fuel zone on the south side of the plate for plates irradiated in Cycle 150B, MOC1 (18.0 EFPD).

Table 27: As-run minimum, maximum and average plate surface temperatures over fuel zone on the north side of the plate for plates irradiated in Cycle 150B, MOC1 (18.0 EFPD)

Table 28: As-run minimum, maximum and average plate surface temperatures over fuel zone on the south side of the plate for plates irradiated in Cycle 150B, MOC2 (31.0 EFPD).

Table 29: As-run minimum, maximum and average plate surface temperatures over fuel zone on the north side of the plate for plates irradiated in Cycle 150B, MOC2 (31.0 EFPD).....

Table 30: As-run minimum, maximum and average plate surface temperatures over fuel zone on the south side of the plate for plates irradiated in Cycle 150B, EOC (41.9 EFPD)

Table 31: As-run minimum, maximum and average plate surface temperatures over fuel zone on the north side of the plate for plates irradiated in Cycle 150B, EOC (41.9 EFPD)

Table 32: As-run minimum, maximum and average plate surface temperatures over fuel zone on the south side of the plate for plates irradiated in Cycle 151A, BOC (0.0 EFPD)

Table 33: As-run minimum, maximum and average plate surface temperatures over fuel zone on the north side of the plate for plates irradiated in Cycle 151A, BOC (0.0 EFPD) .50

Table 34: As-run minimum, maximum and average plate surface temperatures over fuel zone on the south side of the plate for plates irradiated in Cycle 151A, MOC1 (15.0 EFPD)

Table 35: As-run minimum, maximum and average plate surface temperatures over fuel zone on the north side of the plate for plates irradiated in Cycle 151A, MOC1 (15.0 EFPD). 52

Table 36: As-run minimum, maximum and average plate surface temperatures over fuel zone on the south side of the plate for plates irradiated in Cycle 151A, MOC2 (34.0 EFPD) 53

Table 37: As-run minimum, maximum and average plate surface temperatures over fuel zone on the north side of the plate for plates irradiated in Cycle 151A, MOC2 (34.0 EFPD). 54

Table 38: As-run minimum, maximum and average plate surface temperatures over fuel zone on the south side of the plate for plates irradiated in Cycle 151A, EOC (56.1 EFPD) .55 
Table 39: As-run minimum, maximum and average plate surface temperatures over fuel zone on the north side of the plate for plates irradiated in Cycle 151A, EOC (56.1 EFPD).

Table 40: As-run minimum, maximum and average plate surface temperatures over fuel zone on the north side of the plate for plates irradiated in Cycle 151B, BOC (0.0 EFPD)

Table 41: As-run minimum, maximum and average plate surface temperatures over fuel zone on the south side of the plate for plates irradiated in Cycle 151B, BOC (0.0 EFPD)...

Table 42: As-run minimum, maximum and average plate surface temperatures over fuel zone on the north side of the plate for plates irradiated in Cycle 151B, MOC1 (23.0 EFPD)

Table 43: As-run minimum, maximum and average plate surface temperatures over fuel zone on the south side of the plate for plates irradiated in Cycle 151B, MOC1 (23.0 EFPD)....

Table 44: As-run minimum, maximum and average plate surface temperatures over fuel zone on the north side of the plate for plates irradiated in Cycle 151B, MOC2 (39.0 EFPD).

Table 45: As-run minimum, maximum and average plate surface temperatures over fuel zone on the south side of the plate for plates irradiated in Cycle 151B, MOC2 (39.0 EFPD).

Table 46: As-run minimum, maximum and average plate surface temperatures over fuel zone on the north side of the plate for plates irradiated in Cycle 151B, EOC (51.3 EFPD)

Table 47: As-run minimum, maximum and average plate surface temperatures over fuel zone on the south side of the plate for plates irradiated in Cycle 151B, EOC (51.3 EFPD) .59 


\section{ACRONYMS}

$\begin{array}{ll}\text { Al } & \text { Aluminum } \\ \text { ATR } & \text { Advanced Test Reactor } \\ \text { BOC } & \text { Beginning of Cycle } \\ \text { DAS } & \text { Data Acquisition System } \\ \text { EFPD } & \text { Effective Full Power Days } \\ \text { EOC } & \text { End of Cycle } \\ \text { FD } & \text { Fuel Development } \\ \text { GTRI } & \text { Global Threat Reduction Initiative } \\ \text { HIP } & \text { Hot Isostatic Pressing } \\ \text { L2AR } & \text { Local-to-Average Ratio } \\ \text { LEU } & \text { Low Enriched Uranium } \\ \text { MCNP } & \text { Monte Carlo N-Particle } \\ \text { MOC } & \text { Middle of Cycle } \\ \text { Mo } & \text { Molybdenum } \\ \text { RERTR } & \text { Reduced Enrichment Research and Test Reactor } \\ \text { U } & \text { Uranium } \\ \text { U-Mo } & \text { Uranium-Molybdenum Alloy } \\ \text { Zr } & \text { Zirconium }\end{array}$




\section{RERTR-12 Insertion 2 Irradiation Summary Report \\ 1. EXPERIMENT GOALS}

In support of the Global Threat Reduction Initiative (GTRI) Fuel Development (FD) program (historically known as Reduced Enrichment Research and Test Reactor (RERTR)), the RERTR-12 experiment was designed to provide comprehensive information on the performance of uranium-molybdenum (U-Mo) based monolithic fuels for research reactor applications. ${ }^{1}$

The RERTR-12 test assembly holds 4 capsules, designated as A, B, C and D, with A at the top of the assembly and D at the bottom. Each capsule has 2 levels, with 4 plate positions per level, for a total of 8 plate positions per capsule and 32 plate positions per assembly. Within each capsule the 8 plate positions are azimuthally designated as 1 through 4 in the upper level and 5 through 8 in the lower level. There were three different capsule configurations associated with the RERTR-12 experiment, the loading diagram for the RERTR-12 insertion 2 Experiment Capsule Configuration is shown in Table 1. The experiment matrix for RERTR-12-3 (RERTR-12 third irradiation cycle) is shown in Table 2, the experiment matrix for RERTR-12-4 (RERTR-12 fourth irradiation cycle) is shown in Table 3, and the experiment matrix for RERTR-12-5 (RERTR-12 fifth irradiation cycle) is shown in Table 4. The RERTR-12 mini-plates were oriented with the plate identification number face on to the core (see Figure $1)$.

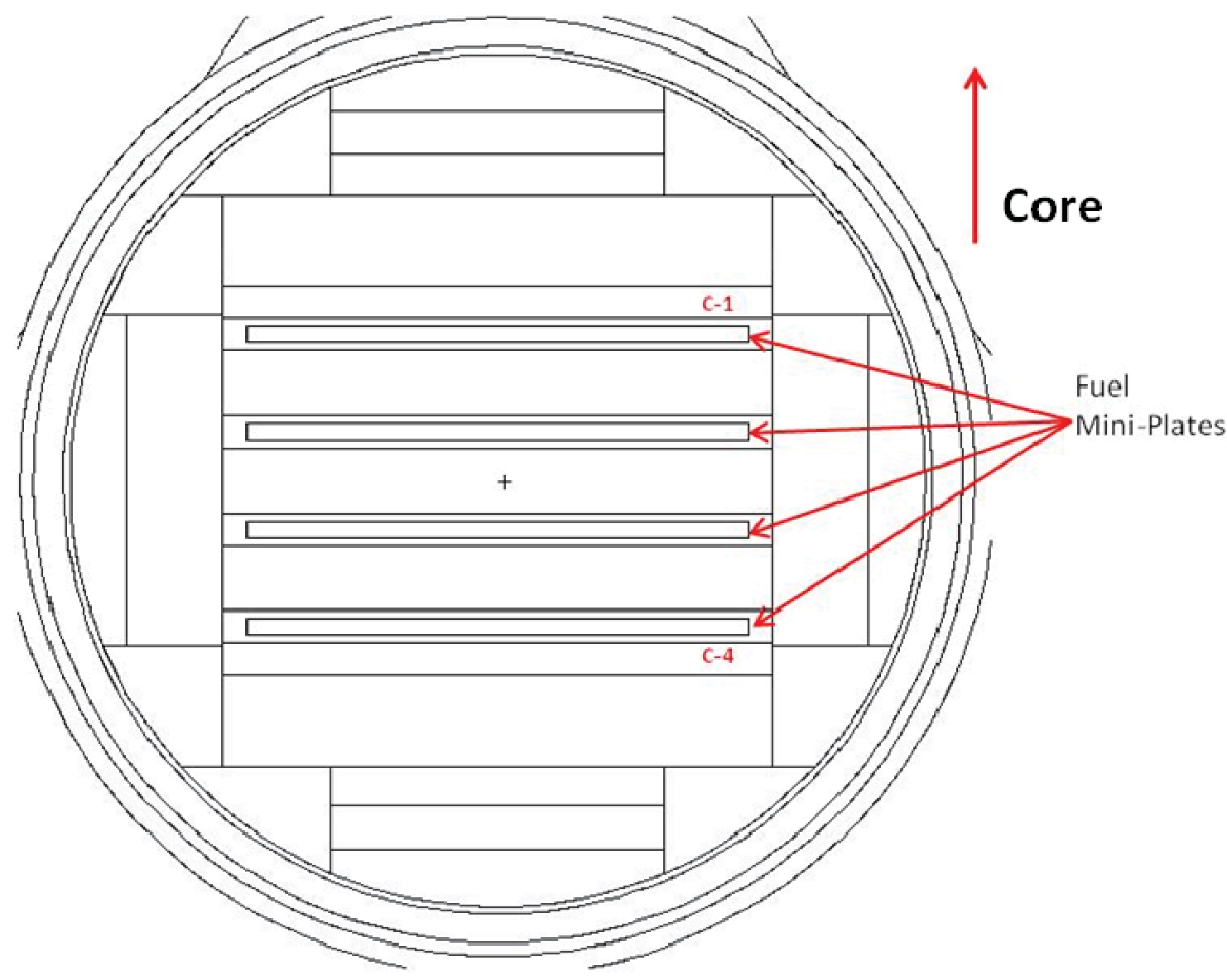

Figure 1: MCNP-Generated radial cross-section view of RERTR-12 test assembly (mini-plates C1 through C4). 
Table 1: RERTR-12 Insertion 2 Experiment Capsule Configuration

\begin{tabular}{|c|c|c|c|}
\hline $\begin{array}{c}\text { RERTR Test } \\
\text { Train } \\
\text { Position } \\
\end{array}$ & $\begin{array}{l}\text { RERTR-12-3 } \\
\text { Capsule }\end{array}$ & $\begin{array}{c}\text { RERTR-12-4 } \\
\text { Capsule }\end{array}$ & $\begin{array}{c}\text { RERTR-12-5 } \\
\text { Capsule }\end{array}$ \\
\hline $\mathrm{A}$ & $\mathrm{Z}\left(2^{\text {nd }}\right.$ Cycle $)$ & $\mathrm{Z}\left(3^{\text {rd }}\right.$ Cycle $)$ & DUM \\
\hline $\mathrm{B}$ & DUM & $\mathrm{Y} 1\left(1^{\text {st }}\right.$ Cycle $)$ & DUM \\
\hline $\mathrm{C}$ & Y3 $\left(1^{\text {st }}\right.$ Cycle $)$ & Y3 $\left(2^{\text {nd }}\right.$ Cycle $)$ & DUM \\
\hline $\mathrm{D}$ & Y2 $\left(1^{\text {st }}\right.$ Cycle $)$ & Y2 $\left(2^{\text {nd }}\right.$ Cycle $)$ & Y2 ( $3^{\text {rd }}$ Cycle $)$ \\
\hline
\end{tabular}

Table 2: RERTR-12-3 Experiment Matrix

\begin{tabular}{|c|c|c|c|c|}
\hline Capsule & Column 1 & Column 2 & Column 3 & Column 4 \\
\hline \multirow[b]{2}{*}{ A-Top } & A1 & A2 & A3 & A4 \\
\hline & $\begin{array}{c}\text { U-10Mo } \\
\text { 70\% Enriched } \\
\text { HIP } \\
\text { L1P787 }\end{array}$ & $\begin{array}{c}\text { U-10Mo } \\
40 \% \text { Enriched } \\
\text { HIP } \\
\text { L2P481 }\end{array}$ & $\begin{array}{c}\text { U-10Mo } \\
40 \% \text { Enriched } \\
\text { HIP } \\
\text { L2P498 }\end{array}$ & $\begin{array}{c}\text { U-10Mo } \\
47 \% \text { Enriched } \\
\text { HIP } \\
\text { L1P789 }\end{array}$ \\
\hline \multirow[b]{2}{*}{ A-Bottom } & A5 & A6 & A7 & A8 \\
\hline & $\begin{array}{c}\text { U-10Mo } \\
\text { 70\% Enriched } \\
\text { HIP } \\
\text { L1P7A0 }\end{array}$ & $\begin{array}{c}\text { U-10Mo } \\
40 \% \text { Enriched } \\
\text { HIP } \\
\text { L2P482 }\end{array}$ & $\begin{array}{c}\text { U-10Mo } \\
40 \% \text { Enriched } \\
\text { HIP } \\
\text { L2P499 }\end{array}$ & $\begin{array}{c}\text { U-10Mo } \\
\text { 70\% Enriched } \\
\text { HIP } \\
\text { L1P7A1 }\end{array}$ \\
\hline \multirow{2}{*}{ B-Тop } & B1 & B2 & B3 & B4 \\
\hline & BLANK & BLANK & BLANK & BLANK \\
\hline \multirow{2}{*}{ B-Bottom } & B5 & B6 & B7 & B8 \\
\hline & BLANK & BLANK & BLANK & BLANK \\
\hline \multirow[b]{2}{*}{ C-Top } & C1 & $\mathrm{C} 2$ & C3 & $\mathrm{C} 4$ \\
\hline & $\begin{array}{c}\text { U-10Mo } \\
\text { 30\% Enriched } \\
\text { HIP } \\
\text { L5P3B1 }\end{array}$ & $\begin{array}{c}\text { U-10Mo } \\
30 \% \text { Enriched } \\
\text { HIP } \\
\text { L5P3B3 }\end{array}$ & $\begin{array}{c}\text { U-10Mo } \\
\text { 20\% Enriched } \\
\text { HIP } \\
\text { L5P2C9 }\end{array}$ & $\begin{array}{c}\text { U-10Mo } \\
\text { 10\% Enriched } \\
\text { HIP } \\
\text { L5P1A5 }\end{array}$ \\
\hline \multirow[b]{2}{*}{ C-Bottom } & $\mathrm{C5}$ & C6 & C7 & C8 \\
\hline & $\begin{array}{c}\text { U-10Mo } \\
\text { 30\% Enriched } \\
\text { HIP } \\
\text { L5P3B2 }\end{array}$ & $\begin{array}{c}\text { U-10Mo } \\
30 \% \text { Enriched } \\
\text { HIP } \\
\text { L5P3C1 }\end{array}$ & $\begin{array}{c}\text { U-10Mo } \\
20 \% \text { Enriched } \\
\text { HIP } \\
\text { L5P2C8 }\end{array}$ & $\begin{array}{c}\text { U-10Mo } \\
10 \% \text { Enriched } \\
\text { HIP } \\
\text { L5P1B8 }\end{array}$ \\
\hline \multirow[b]{2}{*}{ D-Top } & D1 & D2 & D3 & D4 \\
\hline & $\begin{array}{c}\text { U-10Mo } \\
\text { 30\% Enriched } \\
\text { HIP } \\
\text { L5P3C2 }\end{array}$ & $\begin{array}{c}\text { U-10Mo } \\
30 \% \text { Enriched } \\
\text { HIP } \\
\text { L5P3B4 }\end{array}$ & $\begin{array}{c}\text { U-10Mo } \\
20 \% \text { Enriched } \\
\text { HIP } \\
\text { L5P2A3 }\end{array}$ & $\begin{array}{c}\text { U-10Mo } \\
10 \% \text { Enriched } \\
\text { HIP } \\
\text { L5P1B7 }\end{array}$ \\
\hline \multirow[b]{2}{*}{ D-Bottom } & D5 & D6 & D7 & D8 \\
\hline & $\begin{array}{c}\text { U-10Mo } \\
30 \% \text { Enriched } \\
\text { HIP } \\
\text { L5P3C3 }\end{array}$ & $\begin{array}{c}\text { U-10Mo } \\
30 \% \text { Enriched } \\
\text { HIP } \\
\text { L5P3C6 }\end{array}$ & $\begin{array}{c}\text { U-10Mo } \\
20 \% \text { Enriched } \\
\text { HIP } \\
\text { L5P2C0 }\end{array}$ & $\begin{array}{c}\text { U-10Mo } \\
10 \% \text { Enriched } \\
\text { HIP } \\
\text { L5P1B9 }\end{array}$ \\
\hline
\end{tabular}


Table 3: RERTR-12-4 Experiment Matrix

\begin{tabular}{|c|c|c|c|c|}
\hline Capsule & Column 1 & Column 2 & Column 3 & Column 4 \\
\hline \multirow[b]{2}{*}{ A-Тop } & A1 & $\mathbf{A} 2$ & A3 & A4 \\
\hline & $\begin{array}{c}\text { U-10Mo } \\
\text { 70\% Enriched } \\
\text { HIP } \\
\text { L1P787 } \\
\end{array}$ & $\begin{array}{c}\text { U-10Mo } \\
40 \% \text { Enriched } \\
\text { HIP } \\
\text { L2P481 }\end{array}$ & $\begin{array}{c}\text { U-10Mo } \\
40 \% \text { Enriched } \\
\text { HIP } \\
\text { L2P498 }\end{array}$ & $\begin{array}{c}\text { U-10Mo } \\
47 \% \text { Enriched } \\
\text { HIP } \\
\text { L1P789 } \\
\end{array}$ \\
\hline \multirow[b]{2}{*}{ A-Bottom } & A5 & A6 & A7 & A8 \\
\hline & $\begin{array}{c}\text { U-10Mo } \\
\text { 70\% Enriched } \\
\text { HIP } \\
\text { L1P7A0 }\end{array}$ & $\begin{array}{c}\text { U-10Mo } \\
40 \% \text { Enriched } \\
\text { HIP } \\
\text { L2P482 }\end{array}$ & $\begin{array}{c}\text { U-10Mo } \\
40 \% \text { Enriched } \\
\text { HIP } \\
\text { L2P499 }\end{array}$ & $\begin{array}{c}\text { U-10Mo } \\
\text { 70\% Enriched } \\
\text { HIP } \\
\text { L1P7A1 }\end{array}$ \\
\hline \multirow[b]{2}{*}{ B-Top } & B1 & B2 & B3 & B4 \\
\hline & $\begin{array}{c}\text { U-10Mo } \\
30 \% \text { Enriched } \\
\text { HIP } \\
\text { L5P3F8 }\end{array}$ & $\begin{array}{c}\text { U-10Mo } \\
30 \% \text { Enriched } \\
\text { HIP } \\
\text { L5P3G2 }\end{array}$ & $\begin{array}{c}\text { U-10Mo } \\
20 \% \text { Enriched } \\
\text { HIP } \\
\text { L5P2A4 }\end{array}$ & $\begin{array}{c}\text { U-10Mo } \\
10 \% \text { Enriched } \\
\text { HIP } \\
\text { L5P1B5 }\end{array}$ \\
\hline \multirow[b]{2}{*}{ B-Bottom } & B5 & B6 & B7 & B8 \\
\hline & $\begin{array}{c}\text { U-10Mo } \\
30 \% \text { Enriched } \\
\text { HIP } \\
\text { L5P3F0 }\end{array}$ & $\begin{array}{c}\text { U-10Mo } \\
30 \% \text { Enriched } \\
\text { HIP } \\
\text { L5P3G3 }\end{array}$ & $\begin{array}{c}\text { U-10Mo } \\
20 \% \text { Enriched } \\
\text { HIP } \\
\text { L5P2C7 }\end{array}$ & $\begin{array}{c}\text { U-10Mo } \\
10 \% \text { Enriched } \\
\text { HIP } \\
\text { L5P1B0 }\end{array}$ \\
\hline \multirow[b]{2}{*}{ C-Top } & C1 & $\mathrm{C} 2$ & $\mathrm{C} 3$ & $\mathrm{C} 4$ \\
\hline & $\begin{array}{c}\text { U-10Mo } \\
\text { 30\% Enriched } \\
\text { HIP } \\
\text { L5P3B1 }\end{array}$ & $\begin{array}{c}\text { U-10Mo } \\
30 \% \text { Enriched } \\
\text { HIP } \\
\text { L5P3B3 }\end{array}$ & $\begin{array}{c}\text { U-10Mo } \\
20 \% \text { Enriched } \\
\text { HIP } \\
\text { L5P2C9 }\end{array}$ & $\begin{array}{c}\text { U-10Mo } \\
10 \% \text { Enriched } \\
\text { HIP } \\
\text { L5P1A5 }\end{array}$ \\
\hline \multirow[b]{2}{*}{ C-Bottom } & C5 & C6 & $\mathrm{C} 7$ & $\mathrm{C} 8$ \\
\hline & $\begin{array}{c}\text { U-10Mo } \\
\text { 30\% Enriched } \\
\text { HIP } \\
\text { L5P3B2 }\end{array}$ & $\begin{array}{c}\text { U-10Mo } \\
30 \% \text { Enriched } \\
\text { HIP } \\
\text { L5P3C1 }\end{array}$ & $\begin{array}{c}\text { U-10Mo } \\
20 \% \text { Enriched } \\
\text { HIP } \\
\text { L5P2C8 }\end{array}$ & $\begin{array}{c}\text { U-10Mo } \\
10 \% \text { Enriched } \\
\text { HIP } \\
\text { L5P1B8 }\end{array}$ \\
\hline \multirow[b]{2}{*}{ D-Top } & D1 & D2 & D3 & D4 \\
\hline & $\begin{array}{c}\text { U-10Mo } \\
30 \% \text { Enriched } \\
\text { HIP } \\
\text { L5P3C2 }\end{array}$ & $\begin{array}{c}\text { U-10Mo } \\
30 \% \text { Enriched } \\
\text { HIP } \\
\text { L5P3B4 }\end{array}$ & $\begin{array}{c}\text { U-10Mo } \\
20 \% \text { Enriched } \\
\text { HIP } \\
\text { L5P2A3 }\end{array}$ & $\begin{array}{c}\text { U-10Mo } \\
10 \% \text { Enriched } \\
\text { HIP } \\
\text { L5P1B7 }\end{array}$ \\
\hline \multirow[b]{2}{*}{ D-Bottom } & D5 & D6 & D7 & D8 \\
\hline & $\begin{array}{c}\text { U-10Mo } \\
30 \% \text { Enriched } \\
\text { HIP } \\
\text { L5P3C3 }\end{array}$ & $\begin{array}{c}\text { U-10Mo } \\
30 \% \text { Enriched } \\
\text { HIP } \\
\text { L5P3C6 }\end{array}$ & $\begin{array}{c}\text { U-10Mo } \\
20 \% \text { Enriched } \\
\text { HIP } \\
\text { L5P2C0 }\end{array}$ & $\begin{array}{c}\text { U-10Mo } \\
10 \% \text { Enriched } \\
\text { HIP } \\
\text { L5P1B9 }\end{array}$ \\
\hline
\end{tabular}


Table 4: RERTR-12-5 Experiment Matrix

\begin{tabular}{|c|c|c|c|c|}
\hline Capsule & Column 1 & Column 2 & Column 3 & Column 4 \\
\hline \multirow{2}{*}{ A-Top } & A1 & A2 & A3 & A4 \\
\hline & BLANK & BLANK & BLANK & BLANK \\
\hline \multirow{2}{*}{ A-Bottom } & A5 & A6 & A7 & A8 \\
\hline & BLANK & BLANK & BLANK & BLANK \\
\hline \multirow{2}{*}{ B-Top } & B1 & B2 & B3 & B4 \\
\hline & BLANK & BLANK & BLANK & BLANK \\
\hline \multirow{2}{*}{ B-Bottom } & B5 & B6 & B7 & B8 \\
\hline & BLANK & BLANK & BLANK & BLANK \\
\hline \multirow{2}{*}{ C-Top } & C1 & $\mathrm{C} 2$ & $\mathrm{C} 3$ & $\mathrm{C} 4$ \\
\hline & BLANK & BLANK & BLANK & BLANK \\
\hline \multirow{2}{*}{ C-Bottom } & C5 & C6 & C7 & C8 \\
\hline & BLANK & BLANK & BLANK & BLANK \\
\hline \multirow[b]{2}{*}{ D-Top } & D1 & D2 & D3 & D4 \\
\hline & $\begin{array}{c}\text { U-10Mo } \\
\text { 30\% Enriched } \\
\text { HIP } \\
\text { L5P3C2 }\end{array}$ & $\begin{array}{c}\text { U-10Mo } \\
30 \% \text { Enriched } \\
\text { HIP } \\
\text { L5P3B4 }\end{array}$ & $\begin{array}{c}\text { U-10Mo } \\
20 \% \text { Enriched } \\
\text { HIP } \\
\text { L5P2A3 }\end{array}$ & $\begin{array}{c}\text { U-10Mo } \\
\text { 10\% Enriched } \\
\text { HIP } \\
\text { L5P1B7 }\end{array}$ \\
\hline \multirow[b]{2}{*}{ D-Bottom } & D5 & D6 & D7 & D8 \\
\hline & $\begin{array}{c}\text { U-10Mo } \\
\text { 30\% Enriched } \\
\text { HIP } \\
\text { L5P3C3 }\end{array}$ & $\begin{array}{c}\text { U-10Mo } \\
\text { 30\% Enriched } \\
\text { HIP } \\
\text { L5P3C6 }\end{array}$ & $\begin{array}{c}\text { U-10Mo } \\
20 \% \text { Enriched } \\
\text { HIP } \\
\text { L5P2C0 }\end{array}$ & $\begin{array}{c}\text { U-10Mo } \\
10 \% \text { Enriched } \\
\text { HIP } \\
\text { L5P1B9 }\end{array}$ \\
\hline
\end{tabular}




\section{CONSTITUENT MASSES AND DENSITIES}

The constituent masses and densities for plates in the Z, Y1, Y2, and Y3 capsules were obtained from the as-built package plate summary sheets ${ }^{2,3}$. Table 5 summarizes the constituent mass and density for all plates irradiated in RERTR-12-3, RERTR-12-4, and RERTR-12-5.

Table 5: RERTR-12 Constituent masses and densities for plates irradiated in the last three cycles

\begin{tabular}{|c|c|c|c|c|c|c|c|c|}
\hline \multirow{2}{*}{$\begin{array}{c}\text { Fuel } \\
\text { Plate } \\
\text { ID }\end{array}$} & \multirow[b]{2}{*}{$\begin{array}{c}\text { Fuel Plate } \\
\text { Number }\end{array}$} & \multirow[b]{2}{*}{$\begin{array}{c}\text { Volume } \\
\text { (cc) }\end{array}$} & \multicolumn{3}{|c|}{ Fuel Constituent Masses } & \multicolumn{3}{|c|}{ Constituent Densities } \\
\hline & & & $\begin{array}{c}\text { Total-U } \\
(\mathrm{g})\end{array}$ & $\begin{array}{c}\text { U-235 } \\
(\mathrm{g})\end{array}$ & $\begin{array}{l}\text { Mo } \\
(\mathrm{g})\end{array}$ & $\begin{array}{l}\text { Total U } \\
\text { (g/cc) }\end{array}$ & $\begin{array}{l}\text { U-235 } \\
(\mathrm{g} / \mathrm{cc})\end{array}$ & $\begin{array}{c}\text { Mo } \\
(\mathrm{g} / \mathrm{cc})\end{array}$ \\
\hline Z-1 & L1P787 & 0.3626 & 6.055 & 4.201 & 0.694 & 16.701 & 11.587 & 1.914 \\
\hline $\mathrm{Z}-2$ & L2P481 & 0.8202 & 12.196 & 4.788 & 1.421 & 14.870 & 5.838 & 1.733 \\
\hline Z-3 & L2P498 & 0.7941 & 11.395 & 4.559 & 1.244 & 14.349 & 5.741 & 1.567 \\
\hline Z-4 & L1P789 & 0.4040 & 6.064 & 4.207 & 0.695 & 15.009 & 10.412 & 1.720 \\
\hline Z-5 & L1P7A0 & 0.4013 & 6.162 & 4.291 & 0.692 & 15.356 & 10.693 & 1.724 \\
\hline Z-6 & L2P482 & 0.7996 & 12.193 & 4.820 & 1.379 & 15.250 & 6.028 & 1.725 \\
\hline $\mathrm{Z}-7$ & L2P499 & 0.7937 & 11.031 & 4.413 & 1.205 & 13.898 & 5.560 & 1.518 \\
\hline Z-8 & L1P7A1 & 0.4013 & 6.167 & 4.294 & 0.693 & 15.368 & 10.701 & 1.727 \\
\hline Y1-1 & L5P3F8 & 1.0107 & 15.543 & 4.674 & 10.869 & 15.378 & 4.625 & 1.709 \\
\hline Y1-2 & L5P3G2 & 1.0517 & 15.668 & 4.652 & 11.016 & 14.898 & 4.423 & 1.660 \\
\hline Y1-3 & L5P2A4 & 1.0100 & 15.645 & 3.272 & 12.373 & 15.490 & 3.240 & 1.727 \\
\hline Y1-4 & L5P1B5 & 1.0494 & 15.693 & 1.607 & 14.086 & 14.954 & 1.531 & 1.664 \\
\hline Y1-5 & & 1.0071 & 15.638 & & 11.084 & 15.528 & 4.522 & 1.721 \\
\hline Y1-6 & L5P3G3 & 1.0086 & 15.642 & 4.644 & 10.998 & 15.509 & 4.604 & 1.728 \\
\hline Y1-7 & L5P2C7 & 1.0561 & 15.767 & 3.047 & 12.720 & 14.929 & 2.885 & 1.659 \\
\hline Y1-8 & L5P1B0 & 0.8870 & 13.754 & 1.437 & 12.317 & 15.505 & 1.620 & 1.724 \\
\hline Y2-1 & L5P3C2 & 1.0540 & 15.731 & 4.680 & 11.051 & 14.925 & 4.440 & 1.664 \\
\hline Y2-2 & L5P3B4 & 1.0603 & 15.535 & 4.713 & 10.822 & 14.652 & 4.445 & 1.629 \\
\hline Y2-3 & L5P2A3 & 1.0100 & 15.816 & 3.308 & 12.508 & 15.660 & 3.275 & 1.746 \\
\hline $\mathrm{Y} 2-4$ & L5P1B7 & 1.0087 & 15.670 & 1.637 & 14.033 & 15.534 & 1.623 & 1.727 \\
\hline Y2-5 & L5P3C3 & 1.0076 & 15.508 & 4.554 & 10.954 & 15.391 & 4.520 & 1.708 \\
\hline Y2-6 & L5P3C6 & 0.9714 & 15.774 & 4.620 & 11.154 & 16.238 & 4.756 & 1.788 \\
\hline Y2-7 & $\mathrm{L} 5 \mathrm{P} 2 \mathrm{C} 0$ & 1.0962 & 15.944 & 3.136 & 12.808 & 14.545 & 2.861 & 1.623 \\
\hline Y2-8 & L5P1B9 & 0.9986 & 15.767 & 1.647 & 14.120 & 15.789 & 1.649 & 1.755 \\
\hline Y3-1 & L5P3B1 & 1.0561 & 15.487 & 4.665 & 10.822 & 14.664 & 4.417 & 1.623 \\
\hline Y3-2 & L5P3B3 & 1.0563 & 15.635 & 4.614 & 11.021 & 14.802 & 4.368 & 1.645 \\
\hline Y3-3 & L5P2C9 & 1.0556 & 15.794 & 3.107 & 12.687 & 14.962 & 2.943 & 1.669 \\
\hline Y3-4 & L5P1A5 & 1.0063 & 15.767 & 1.647 & 14.120 & 15.668 & 1.637 & 1.742 \\
\hline Y3-5 & L5P3B2 & 1.0551 & 15.535 & 4.585 & 10.950 & 14.723 & 4.345 & 1.637 \\
\hline Y3-6 & L5P3C1 & 1.0541 & 15.690 & 4.668 & 11.022 & 14.885 & 4.429 & 1.660 \\
\hline Y3-7 & L5P2C8 & 1.0556 & 15.782 & 3.050 & 12.732 & 14.951 & 2.889 & 1.661 \\
\hline Y3-8 & L5P1B8 & 1.0097 & 15.752 & 1.646 & 14.106 & 15.601 & 1.630 & 1.734 \\
\hline
\end{tabular}




\section{EXPERIMENT HARDWARE}

The experiment hardware configuration is identical to that used in the RERTR-7A, $-7 \mathrm{~B},-8,-9 \mathrm{~A},-9 \mathrm{~B}$, $10 \mathrm{~A}$ and $-10 \mathrm{~B}$ experiments. A list of irradiation hardware drawings used for analysis is given in Table 6 .

Table 6: RERTR Irradiation Hardware Drawing List.

\begin{tabular}{cl}
\hline $\begin{array}{c}\text { Drawing } \\
\text { Number }\end{array}$ & \multicolumn{1}{c}{ Drawing Title } \\
\hline DWG-630223 & RERTR ATR Large B-Position Irradiation Experiment Assembly \\
DWG-630233 & ATR Large B-Position Basket \\
DWG-630231 & ATR Top Spacer Assembly \\
DWG-630225 & ATR Upper Spacer Assembly \\
DWG-630229 & ATR Bottom Spacer Assembly \\
DWG-630227 & ATR Large B-Position Fuel Capsule Assembly \\
DWG-630237 & Fuel Capsule \\
DWG-630239 & Capsule Cap \\
DWG-630244 & RERTR Mini-Plate \\
DWG-630245 & Fuel Plate, 0.020 Monolithic \\
\hline
\end{tabular}

The RERTR miniplate irradiation assembly, (see Figure 2) shows the main components of the test assembly, which include the bottom spacer, upper and top spacers, experiment capsules and basket. The bottom spacer elevates the experiment capsules to the correct location in the core. The upper and top spacers allow the operators to assure that the experiment is seated fully into the basket. All spacers are similar to the capsule design except the spacers do not have the grooves for the plates. The capsules hold the fuel plates; a capsule cap is welded onto the top of the capsule to keep the plates from sliding out during handling and irradiation. The fuel plate drawings for monolithic and thick monolithic plates (DWG-630244 and DWG-630245, respectively) and RERTR miniplate capsule assembly are shown in Figure 3, Figure 4 and Figure 5, respectively. Each capsule has a notch at the top and a groove at the bottom which allow the capsules to stack and align properly into the core. The basket holds the test assembly in the reactor during irradiation, the notches on the outer wall allow for bypass coolant flow to cool the outer wall. The basket has two guide bars on the inside wall to guide the assembly into the baskets. 


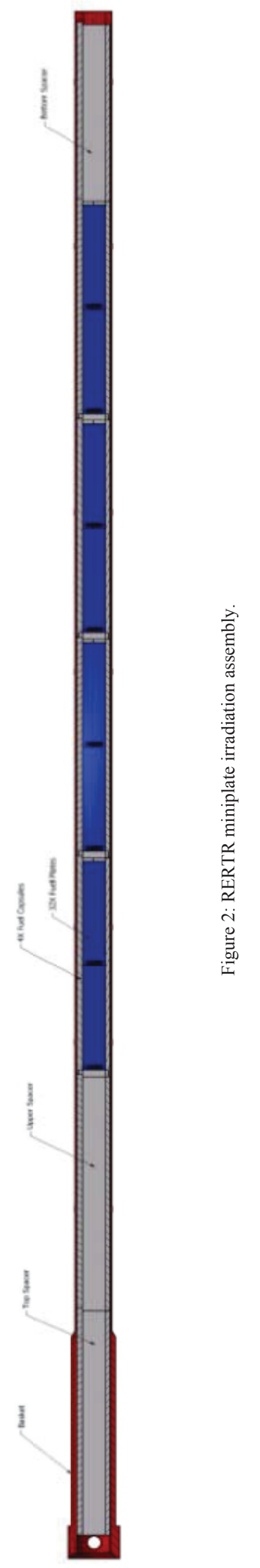




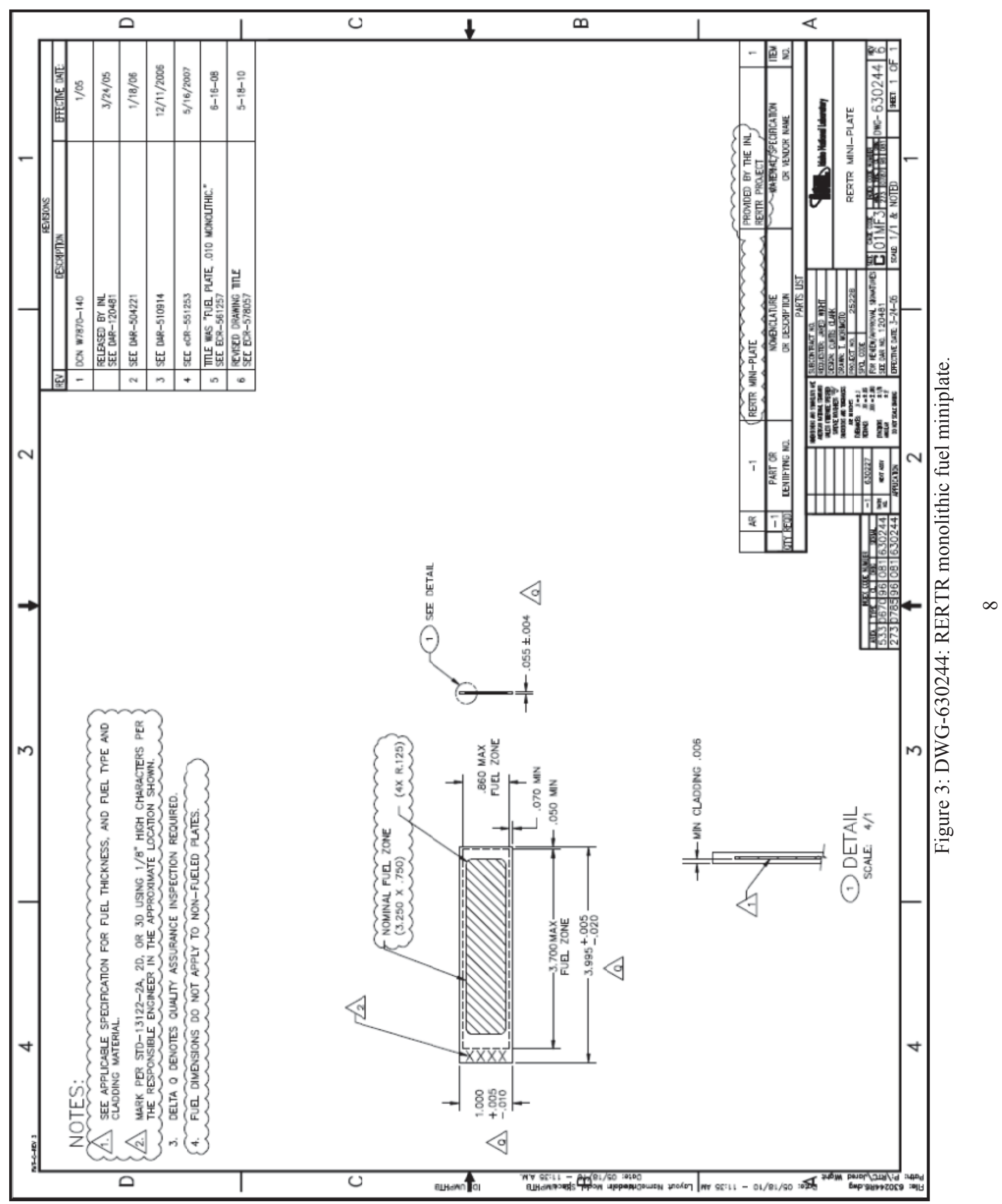




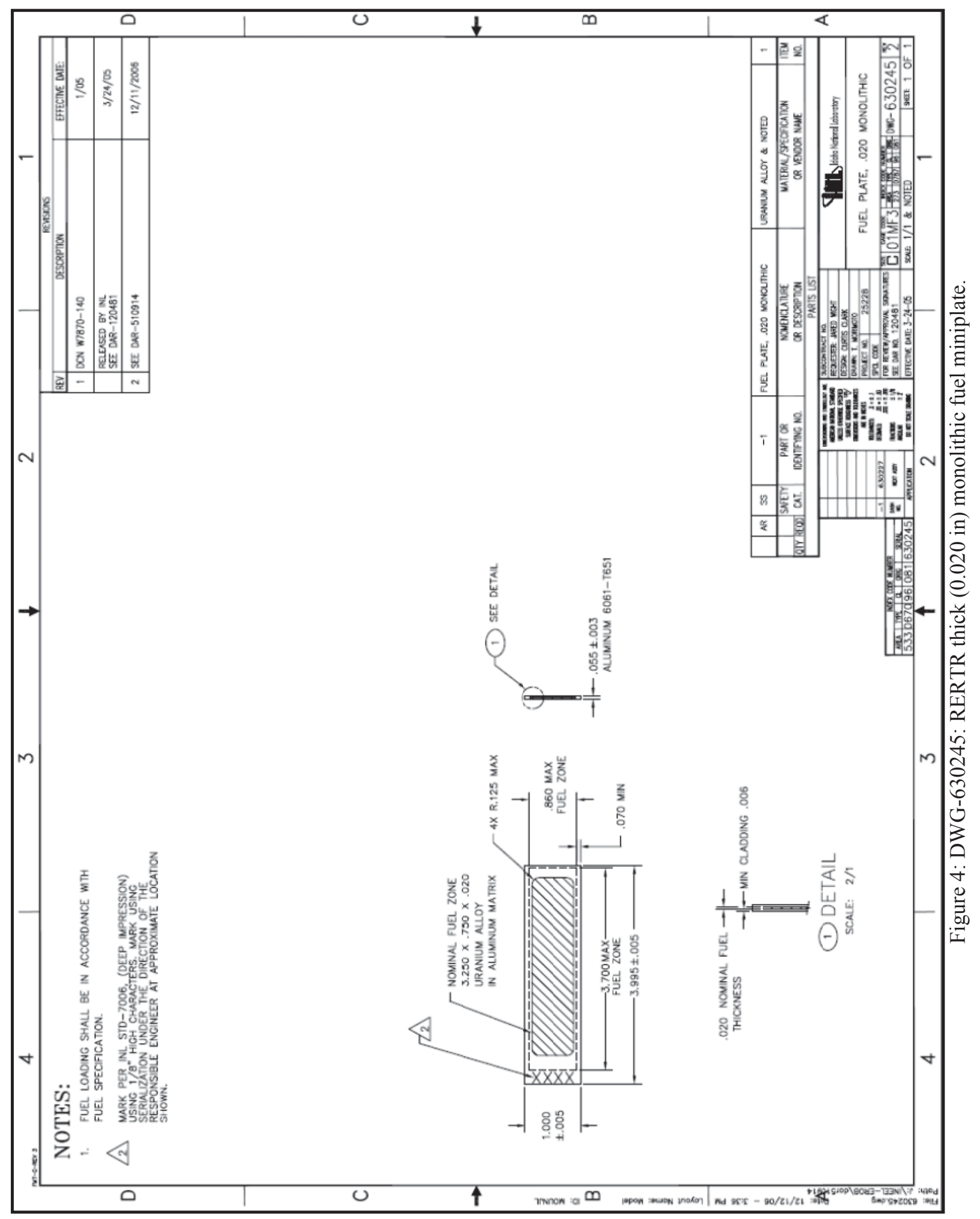




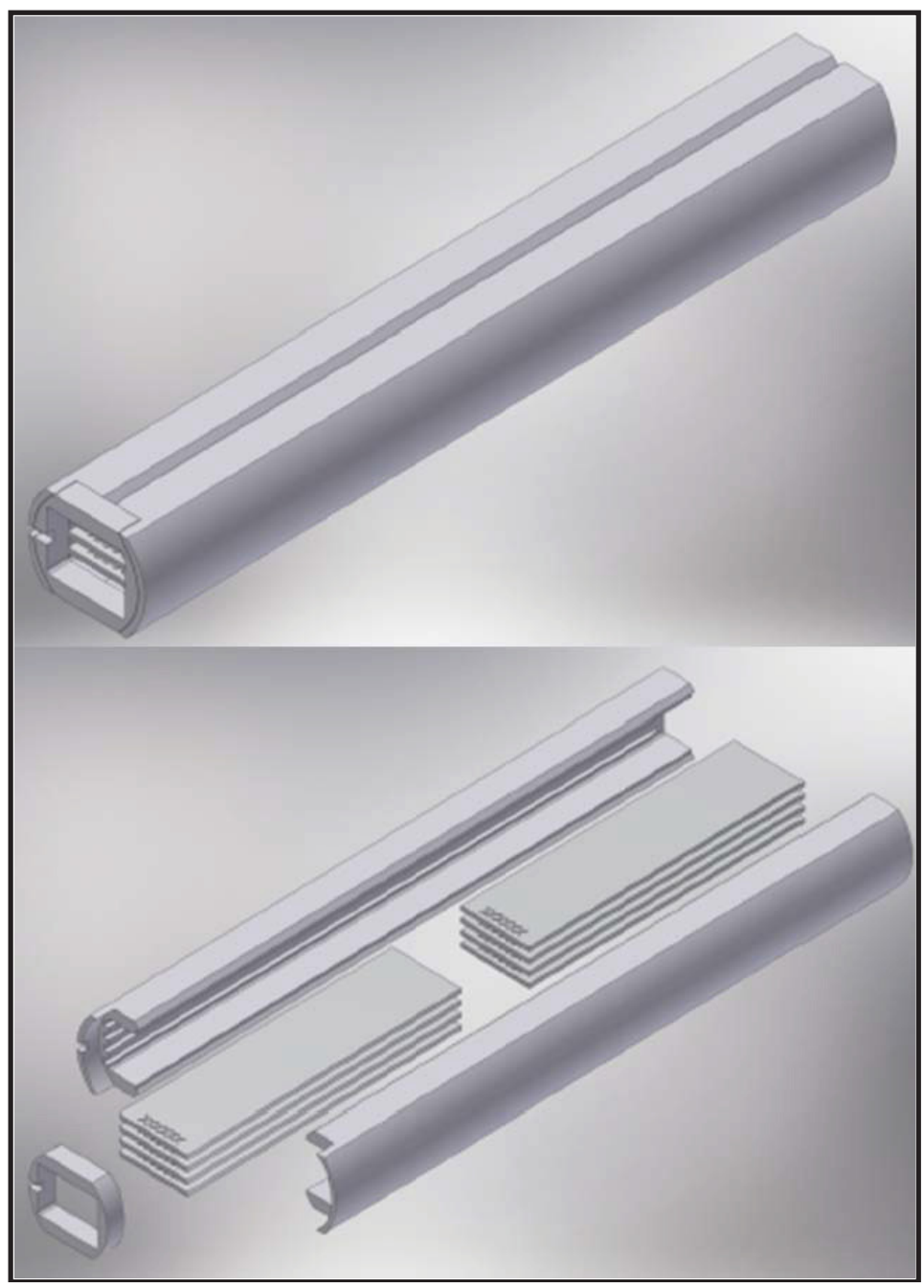

Figure 5: RERTR capsule assembly. 


\section{IRRADIATION HISTORY}

The RERTR-12 insertion 2 test assembly was irradiated in cycle 150B, cycle 151A and cycle 151B. RERTR-12-3 and RERTR-12-4 were irradiated in the large-B position B-9 and RERTR-12-5 was irradiated in the large-B position B-11. The power of position B-9 is represented by the north lobe power which is the average of the NW, $\mathrm{C}$ and NE lobe powers, $\mathrm{N}=(\mathrm{NW}+\mathrm{C}+\mathrm{NE}) / 3$. The power of position B-11 is represented by the south lobe power which is the average of the $\mathrm{SW}, \mathrm{C}$ and $\mathrm{SE}, \mathrm{S}=(\mathrm{SW}+\mathrm{C}+$ $\mathrm{SE}) / 3$. Cycle 150B ran for 41.9 EFPDs at average power of 108.2 MW (north lobe power of 20.7 MW), cycle 151A ran for a total of 56.1 EFPDs at average power of 101.7 MW (north lobe power of 18.4 MW) and cycle 151B ran for a total of 51.3 EFPDs at an average power of 101.5 MW (south lobe power of 22.7 MW).

There were no mid-cycle SCRAMs during Cycle 150B. There was one mid-cycle SCRAM during cycle 151 A with a duration of 3 days from 12/25/2011 - 12/28/2011. There were two mid-cycle SRAMs during cycle $151 \mathrm{~B}$ from $3 / 22 / 2012-3 / 25 / 2012$ and $3 / 27 / 2012$ - 4/7/2012, total duration of 14 days. This information is tabulated in Table 7.

Table 7: Irradiation History for RERTR-12 Insertion 2

\begin{tabular}{|c|c|c|c|c|c|c|c|}
\hline $\begin{array}{c}\text { ATR } \\
\text { CYCLE }\end{array}$ & $\begin{array}{l}\text { RERTR-12 } \\
\text { Capsules } \\
\text { Irradiated* }\end{array}$ & Dates Irradiated & $\begin{array}{l}\text { Cycle } \\
\text { EFPDs }\end{array}$ & $\begin{array}{c}\text { Mid- } \\
\text { Cycle } \\
\text { Scram } \\
\text { Decay } \\
\text { Days }\end{array}$ & $\begin{array}{l}\text { North } \\
\text { Lobe } \\
\text { Source } \\
\text { Power } \\
\text { (MW) }\end{array}$ & $\begin{array}{l}\text { South } \\
\text { Lobe } \\
\text { Source } \\
\text { Power } \\
\text { (MW) }\end{array}$ & $\begin{array}{l}\text { Total } \\
\text { Core } \\
\text { Power } \\
(\mathrm{MW})\end{array}$ \\
\hline $150 \mathrm{~B}$ & A,C,D & $\begin{array}{c}10 / 15 / 2011- \\
11 / 26 / 2011 \\
\end{array}$ & 41.9 & 0 & 20.7 & & 108.2 \\
\hline $151 \mathrm{~A}$ & A,B,C,D & $\begin{array}{c}12 / 14 / 2011- \\
02 / 11 / 2012 \\
\end{array}$ & 56.1 & 3 & 18.4 & & 101.7 \\
\hline $151 \mathrm{~B}$ & D & $\begin{array}{c}03 / 01 / 2012- \\
05 / 05 / 2012\end{array}$ & 51.3 & 14 & & 22.7 & 101.5 \\
\hline
\end{tabular}

*See Table 1 for capsule configurations

The power history for each cycle is obtained as in ATR Surveillance Report from the ATR Data Acquisition System (DAS). The plots of each lobe power on an hourly basis are shown in Figure 6, Figure 7 and Figure 8 for cycle 150B, 151A and 151B, respectively. 


\section{Cycle 150B Hourly Constrained Lobe Powers}

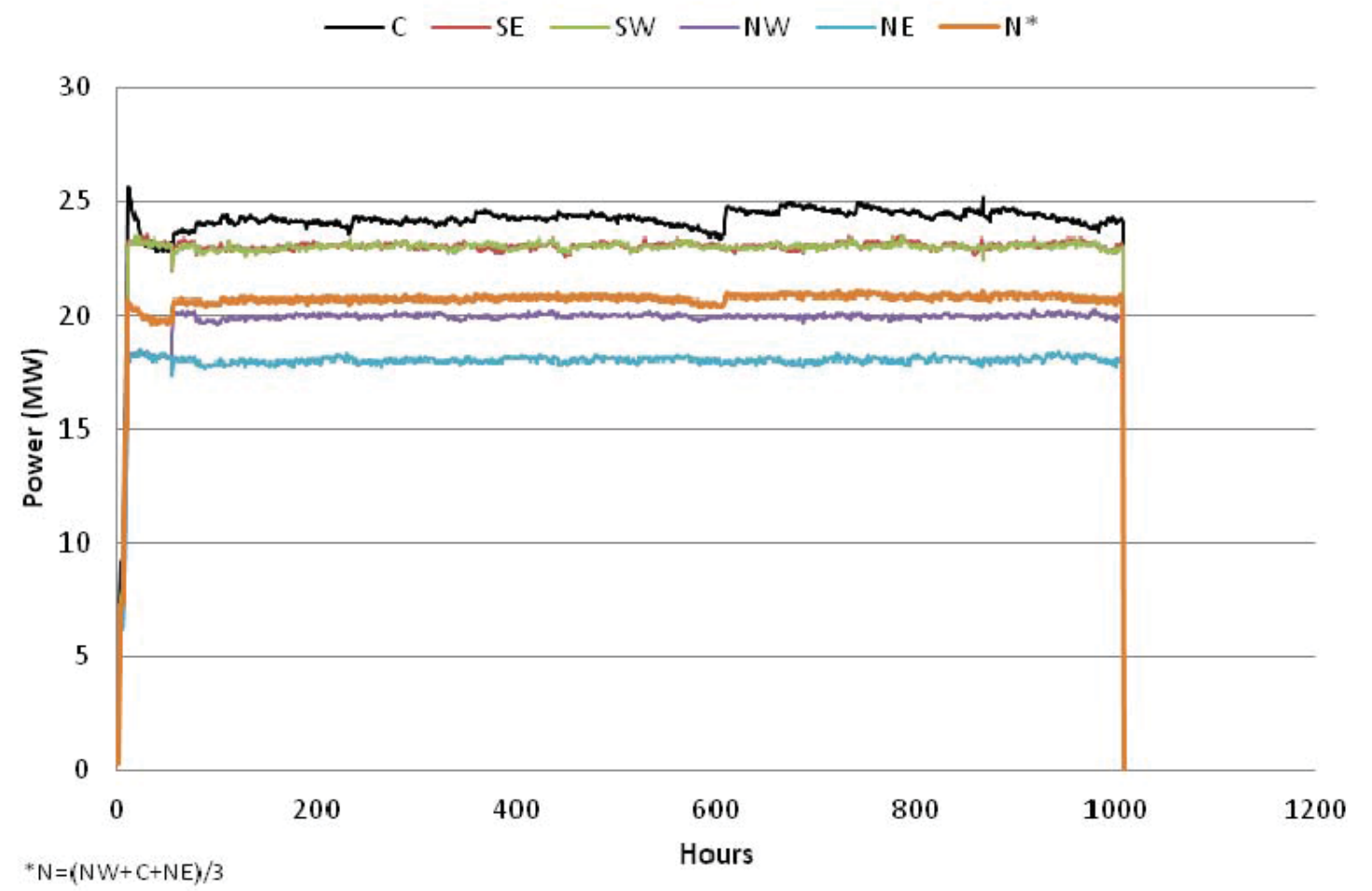

Figure 6: Hourly lobe power history for ATR Cycle 150B.

\section{Cycle 151A Hourly Constrained Lobe Powers}

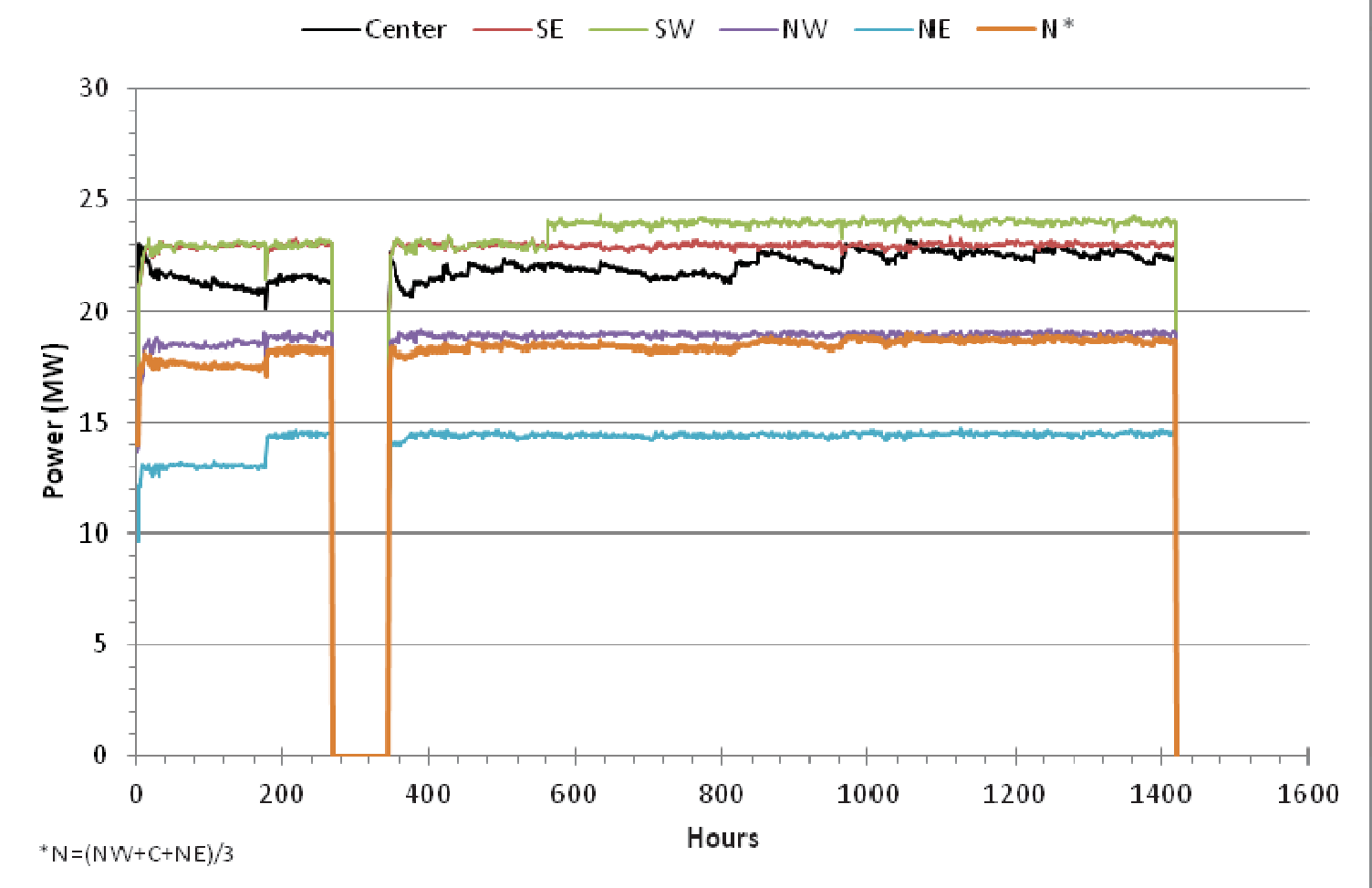

Figure 7: Hourly lobe power history for ATR Cycle 151A. 


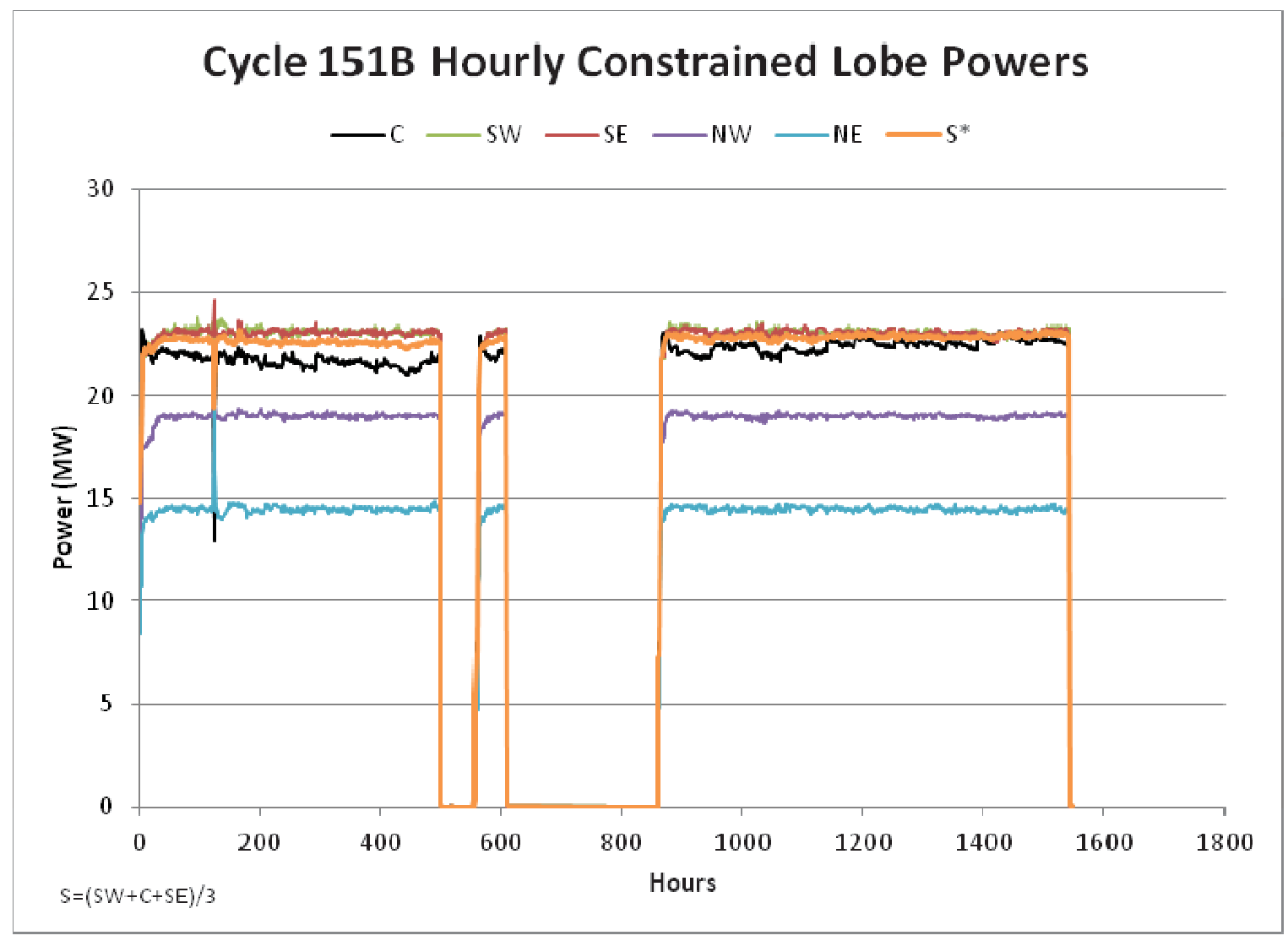

Figure 8. Hourly lobe power history for ATR Cycle 151B. 


\section{AS-RUN NUCLEAR ANALYSIS}

\subsection{Neutronics}

The as-run calculations were performed using the irradiation history in Table 7 and the Monte Carlo N-Particle (MCNP) code. The calculated as-run fission heat rates, fission densities, and asrun U-235 burnup results for the fueled miniplates reported have an uncertainty band $(1 \sigma)$ of $2.5 \%{ }^{4,5,6}$ The time intervals used to calculate the average plate power and burnup is shown in Table 8 . The average plate power and burnup for the time intervals for cycle 150B are shown in Table 11 through Table 14. The average plate power and burnup for the time intervals for cycle 151A are shown in Table 15 through Table 18. The average plate power and burnup for the time intervals for cycle 151B are shown in Table 19 through Table 22. The plots of the power and fission density as a function of the ATR Cycle time interval are in Appendix A.

Table 8: Cycle Breakdown

\begin{tabular}{cccc}
\hline Time Interval & $\begin{array}{c}150 \mathrm{~B} \\
\text { (days) }\end{array}$ & $\begin{array}{c}151 \mathrm{~A} \\
\text { (days) }\end{array}$ & $\begin{array}{c}151 \mathrm{~B} \\
\text { (days) }\end{array}$ \\
\hline BOC & $1.00 \mathrm{E}-04$ & $1.00 \mathrm{E}-04$ & $1.00 \mathrm{e}-4$ \\
\hline MOC 1 & 18.0 & 15.0 & 23.0 \\
\hline MOC 2 & 13.0 & 19.0 & 16.0 \\
\hline EOC & 10.9 & 22.1 & 12.3 \\
\hline Total EFPDs & 41.9 & 56.1 & 51.3 \\
\hline \hline Cumulative & 41.9 & 98.0 & 149.3 \\
\hline
\end{tabular}

The MCNP-calculated neutronic results reported were calculated using the nominal fuel foil mass and thickness shown in Table 9 for plates in type $\mathrm{Z}$ capsule and Table 10 for plates in type $\mathrm{Y}$ capsule.

Table 9: RERTR-12 Calculated Nominal Initial Constituent Masses and Densities for Capsule

Type Z Based off a Nominal Fuel Alloy Density of $17.2 \mathrm{~g} / \mathrm{cc}$

\begin{tabular}{|c|c|c|c|c|c|c|c|c|c|c|c|}
\hline \multirow{2}{*}{$\begin{array}{c}\text { Plate } \\
\text { Position }\end{array}$} & \multirow[b]{2}{*}{ Enrich. } & \multirow{2}{*}{$\begin{array}{c}\text { Fuel Alloy } \\
\begin{array}{c}\text { Thick. } \\
(\mathrm{mm})\end{array}\end{array}$} & \multirow{2}{*}{$\begin{array}{l}\text { Fuel } \\
\text { Alloy } \\
\text { Volume } \\
\text { (cc) }\end{array}$} & \multirow{2}{*}{$\begin{array}{l}\text { Fuel } \\
\text { Alloy } \\
\text { Mass } \\
(\mathrm{g})\end{array}$} & \multicolumn{4}{|c|}{$\begin{array}{l}\text { Fuel Phase Constituent Masses } \\
\text { (g) }\end{array}$} & \multicolumn{3}{|c|}{$\begin{array}{c}\text { Fuel Phase Constituent } \\
\text { Densities } \\
(\mathrm{g} / \mathrm{cc})\end{array}$} \\
\hline & & & & & Total U & U-238 & U-235 & Mo & U-238 & U-235 & Mo \\
\hline 1 & $70 \%$ & 0.254 & 0.399 & 6.870 & 6.183 & 1.855 & 4.328 & 0.687 & 4.644 & 10.836 & 1.720 \\
\hline 2 & $40 \%$ & 0.508 & 0.799 & 13.741 & 12.366 & 7.420 & 4.947 & 1.374 & 9.288 & 6.192 & 1.720 \\
\hline 3 & $40 \%$ & 0.508 & 0.799 & 13.741 & 12.366 & 7.420 & 4.947 & 1.374 & 9.288 & 6.192 & 1.720 \\
\hline 4 & $70 \%$ & 0.254 & 0.399 & 6.870 & 6.183 & 1.855 & 4.328 & 0.687 & 4.644 & 10.836 & 1.720 \\
\hline 5 & $70 \%$ & 0.254 & 0.399 & 6.870 & 6.183 & 1.855 & 4.328 & 0.687 & 4.644 & 10.836 & 1.720 \\
\hline 6 & $40 \%$ & 0.508 & 0.799 & 13.741 & 12.366 & 7.420 & 4.947 & 1.374 & 9.288 & 6.192 & 1.720 \\
\hline 7 & $40 \%$ & 0.508 & 0.799 & 13.741 & 12.366 & 7.420 & 4.947 & 1.374 & 9.288 & 6.192 & 1.720 \\
\hline 8 & $70 \%$ & 0.254 & 0.399 & 6.870 & 6.183 & 1.855 & 4.328 & 0.687 & 4.644 & 10.836 & 1.720 \\
\hline & & & Totals & 82.444 & 74.196 & 37.100 & 37.100 & 8.244 & & & \\
\hline
\end{tabular}


Table 10: RERTR-12 Calculated Nominal Initial Constituent Masses and Densities for Capsule Type Y Based off a Nominal Fuel Alloy Density of $17.2 \mathrm{~g} / \mathrm{cc}$

\begin{tabular}{|c|c|c|c|c|c|c|c|c|c|c|c|}
\hline \multirow{2}{*}{$\begin{array}{c}\text { Plate } \\
\text { Position } \\
\end{array}$} & \multirow[b]{2}{*}{ Enrich. } & \multirow{2}{*}{$\begin{array}{l}\text { Fuel } \\
\text { Alloy } \\
\text { Thick. } \\
(\mathrm{mm}) \\
\end{array}$} & \multirow{2}{*}{$\begin{array}{l}\text { Fuel } \\
\text { Alloy } \\
\text { Volume } \\
\text { (cc) } \\
\end{array}$} & \multirow{2}{*}{$\begin{array}{c}\text { Fuel Alloy } \\
\text { Mass } \\
(\mathrm{g}) \\
\end{array}$} & \multicolumn{4}{|c|}{$\begin{array}{l}\text { Fuel Phase Constituent Masses } \\
\qquad(\mathrm{g})\end{array}$} & \multicolumn{3}{|c|}{$\begin{array}{c}\text { Fuel Phase Constituent } \\
\text { Densities } \\
(\mathrm{g} / \mathrm{cc})\end{array}$} \\
\hline & & & & & Total U & U-238 & U-235 & Mo & U-238 & U-235 & Mo \\
\hline 1 & $30 \%$ & 0.635 & 0.999 & 17.176 & 15.458 & 10.821 & 4.637 & 1.718 & 10.836 & 4.644 & 1.720 \\
\hline 2 & $30 \%$ & 0.635 & 0.999 & 17.176 & 15.458 & 10.821 & 4.637 & 1.718 & 10.836 & 4.644 & 1.720 \\
\hline 3 & $20 \%$ & 0.635 & 0.999 & 17.176 & 15.458 & 12.366 & 3.092 & 1.718 & 12.384 & 3.096 & 1.720 \\
\hline 4 & $10 \%$ & 0.635 & 0.999 & 17.176 & 15.458 & 13.912 & 1.546 & 1.718 & 13.932 & 1.548 & 1.720 \\
\hline 5 & $30 \%$ & 0.635 & 0.999 & 17.176 & 15.458 & 10.821 & 4.637 & 1.718 & 10.836 & 4.644 & 1.720 \\
\hline 6 & $30 \%$ & 0.635 & 0.999 & 17.176 & 15.458 & 10.821 & 4.637 & 1.718 & 10.836 & 4.644 & 1.720 \\
\hline 7 & $20 \%$ & 0.635 & 0.999 & 17.176 & 15.458 & 12.366 & 3.092 & 1.718 & 12.384 & 3.096 & 1.720 \\
\hline 8 & $10 \%$ & 0.635 & 0.999 & 17.176 & 15.458 & 13.912 & 1.546 & 1.718 & 13.932 & 1.548 & 1.720 \\
\hline & & & Totals & 137.408 & 123.664 & 95.840 & 27.824 & 13.744 & & & \\
\hline
\end{tabular}




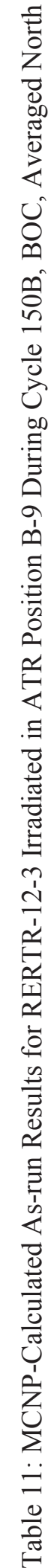

\begin{tabular}{|l|l|l|}
\hline 0 & 0 & \\
0 & 0 & \\
\hline
\end{tabular}

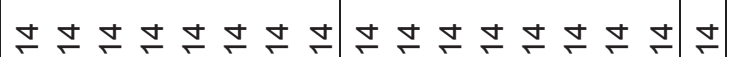

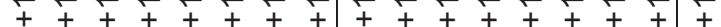

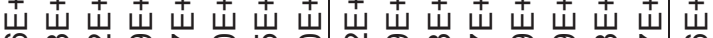
吕 大 $\forall m$ m

mํำ 岕岕占

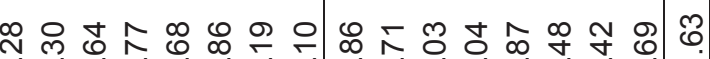

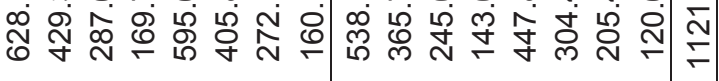

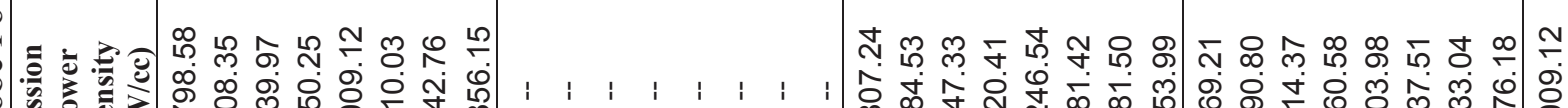

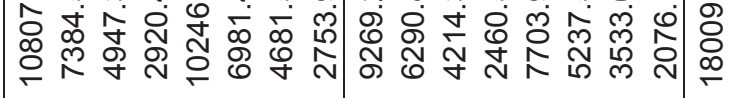

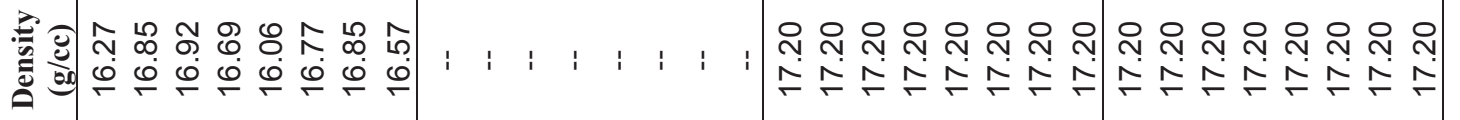
ThF 
告

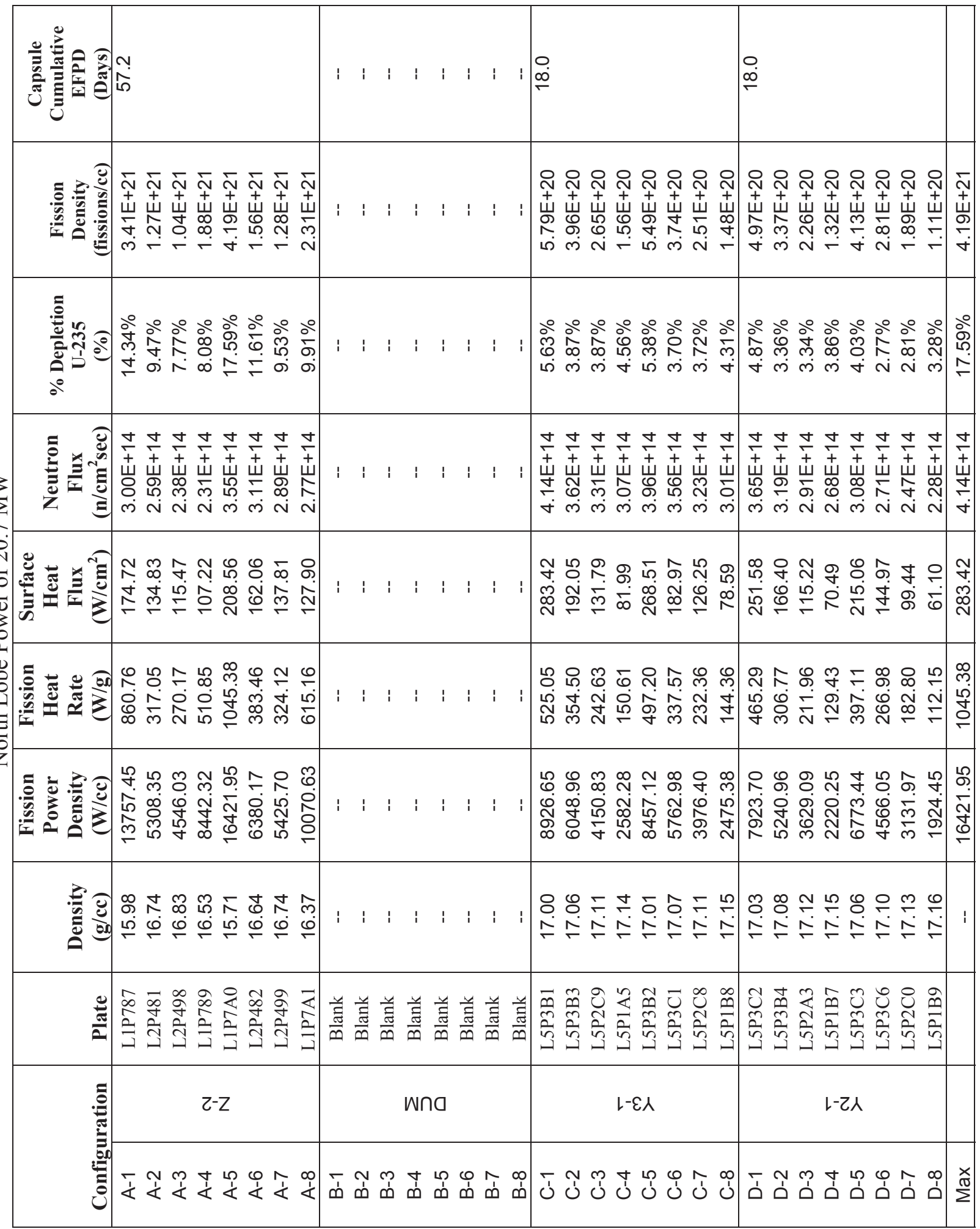




\begin{tabular}{|c|c|c|c|c|}
\hline 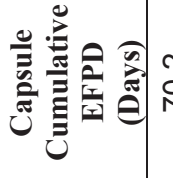 & & $\begin{array}{llllllllllllllll}1 & 1 & 1 & 1 & 1 & 1 & 1 & 1\end{array}$ & 品 & 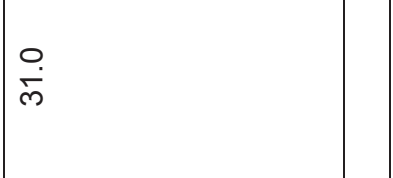 \\
\hline 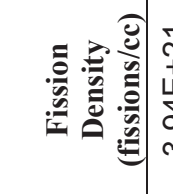 & 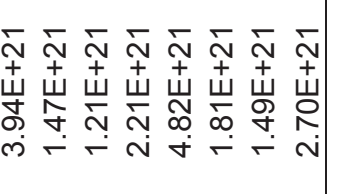 & : : : & 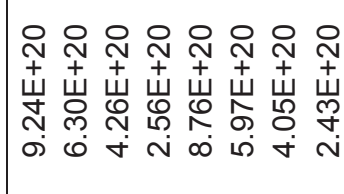 & 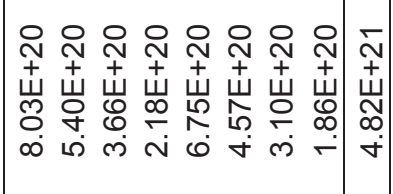 \\
\hline 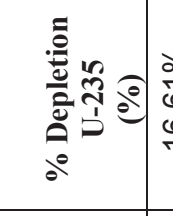 & 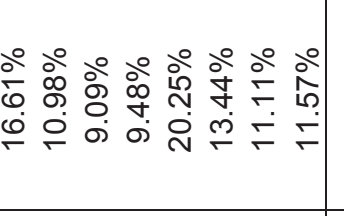 & $1: 1$ & 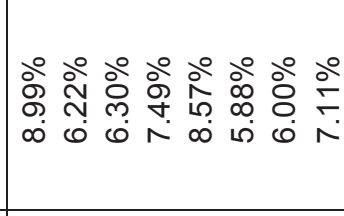 & 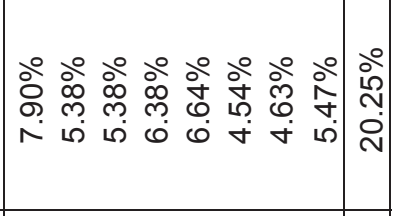 \\
\hline & 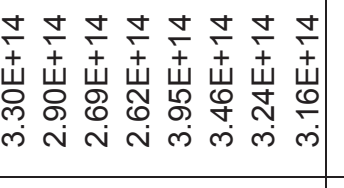 & : : : & 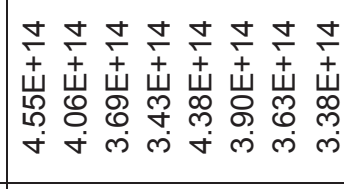 & 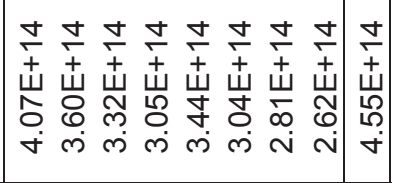 \\
\hline & 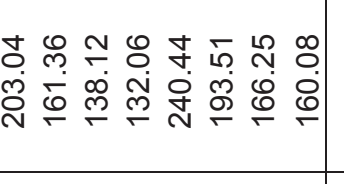 & $1: 1: 1: 1$ & 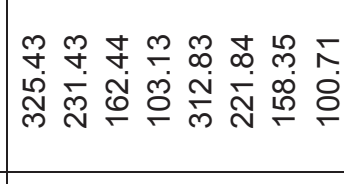 & 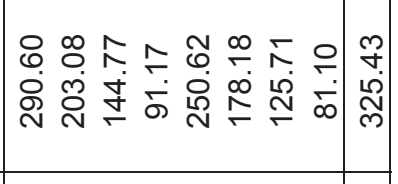 \\
\hline & 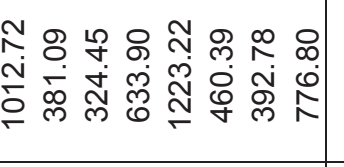 & $1: 1: 1 \quad 1 \quad 1 \quad 11$ & 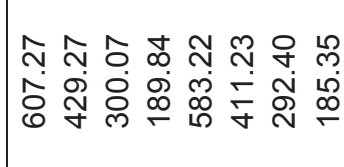 & 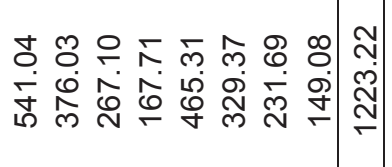 \\
\hline 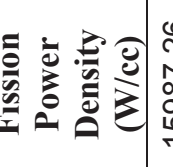 & 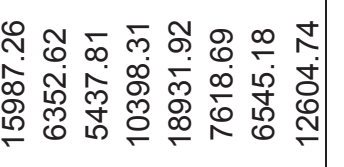 & $1: 1: 1: 1 \quad 1 \quad 1: 1$ & 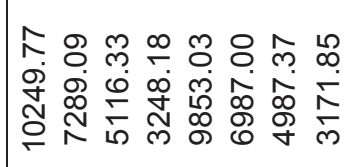 & 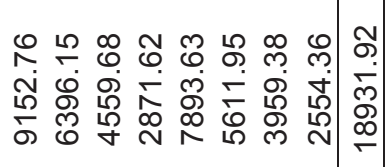 \\
\hline 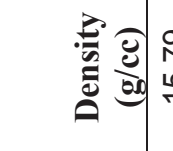 & 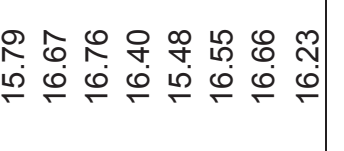 & $1: 1: 1: 1:$ & 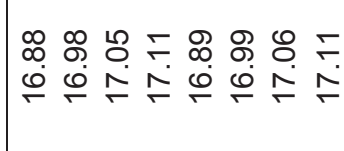 & 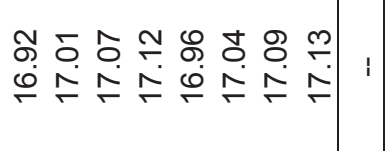 \\
\hline & 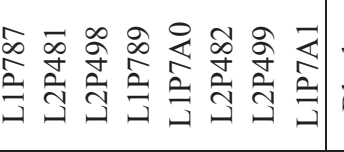 & 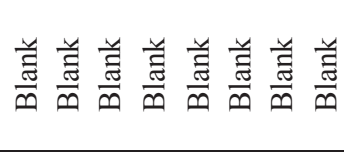 & 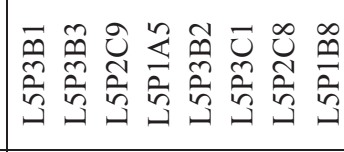 & 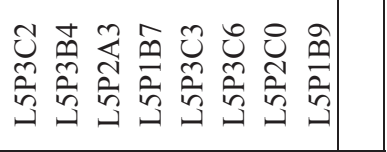 \\
\hline 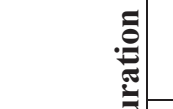 & $z-z$ & wna & $1-\varepsilon \curlywedge$ & $1-2 \lambda$ \\
\hline 韵 & 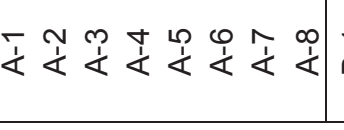 & 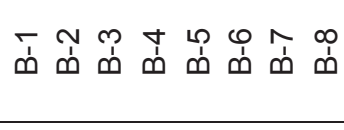 & 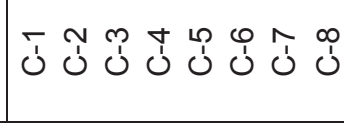 & 돔좀옴봄웅임 \\
\hline
\end{tabular}




\begin{tabular}{|c|c|c|c|c|}
\hline 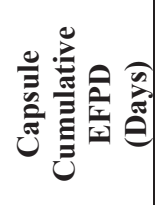 & & $1: 1: 1: 1 \quad 1 \quad 11$ & ใ & ร \\
\hline & 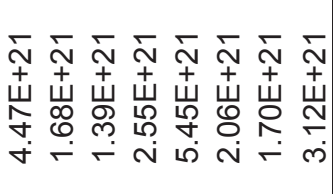 & $: \quad: \quad: \quad:$ & 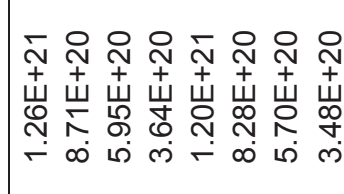 & 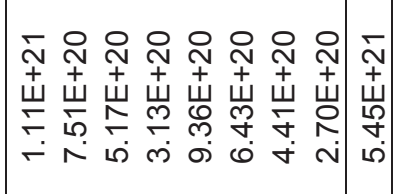 \\
\hline & 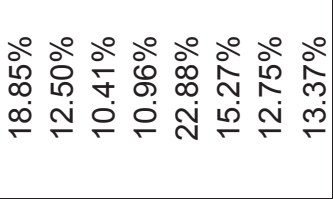 & $1: 1111$ & 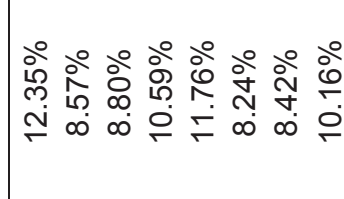 & 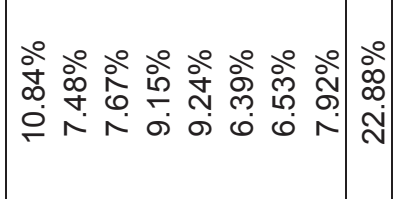 \\
\hline 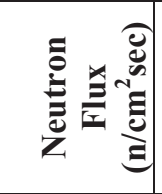 & 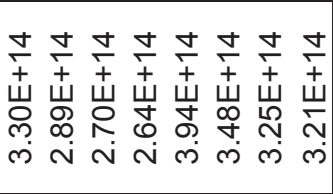 & 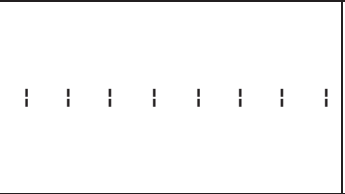 & 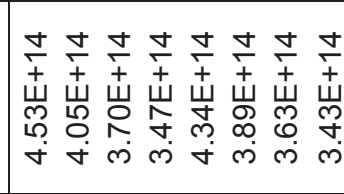 & 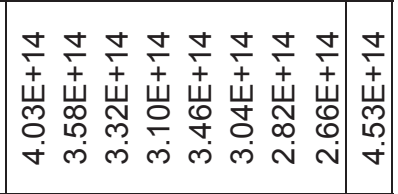 \\
\hline & 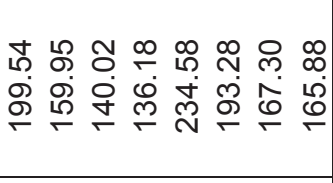 & $:$ & 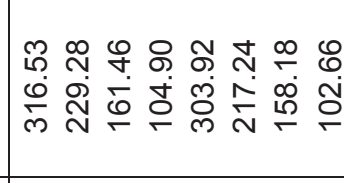 & 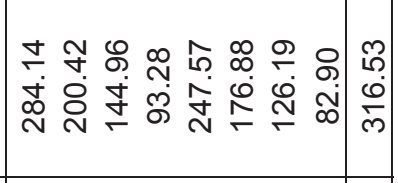 \\
\hline & 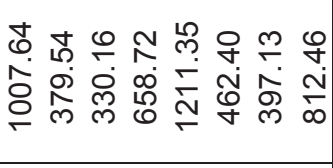 & $1: 1: 1$ & 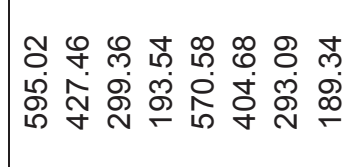 & 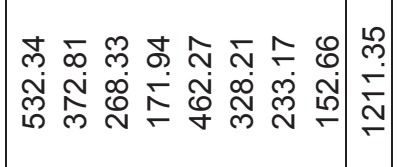 \\
\hline 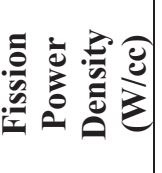 & 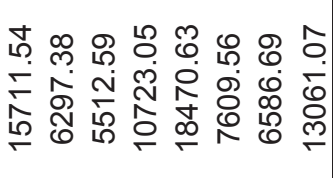 & $1: 1: 1: 1:$ & 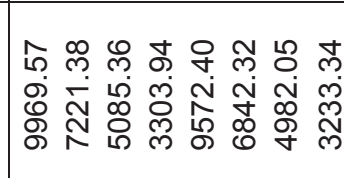 & 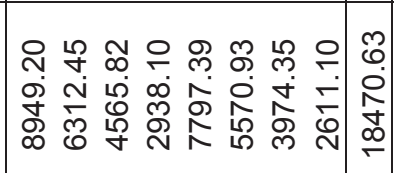 \\
\hline 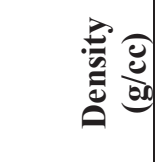 & 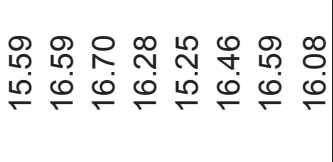 & $1: 1: 1: 1: 1$ & 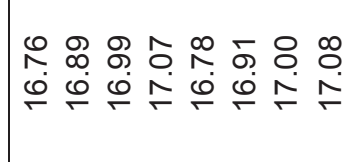 & 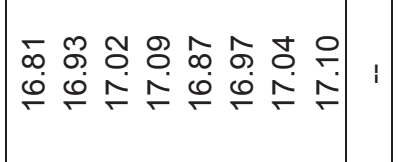 \\
\hline & 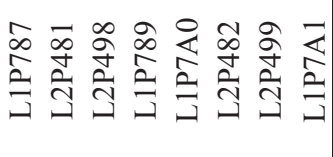 & 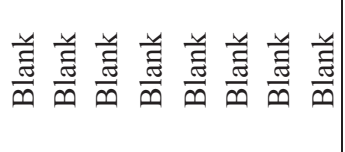 & 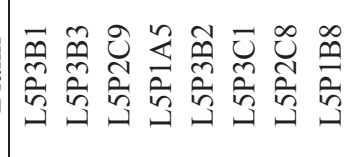 & 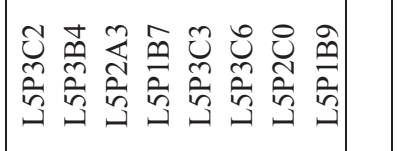 \\
\hline 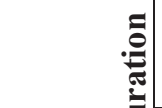 & $z-z$ & wna & $t-\varepsilon \lambda$ & $1-2 \lambda$ \\
\hline & 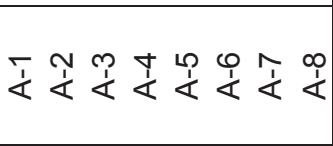 & ஸ் & テ்َ & 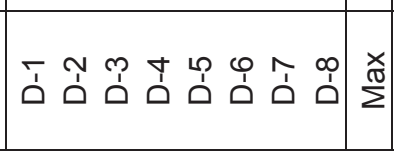 \\
\hline
\end{tabular}


吾 


\begin{tabular}{|c|c|c|c|c|}
\hline 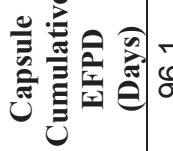 & & $\stackrel{2}{2}$ & $\mid \begin{array}{l}0 \\
\dot{b} \\
i\end{array}$ & $\begin{array}{l}\text { s. } \\
\text { in } \\
\text { in }\end{array}$ \\
\hline & 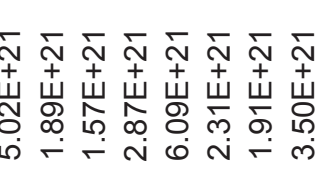 & 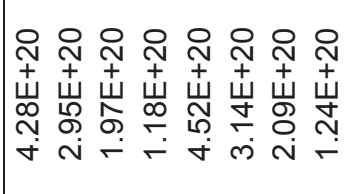 & 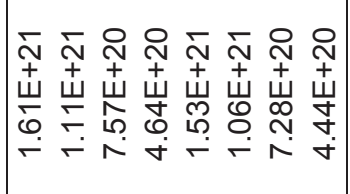 & 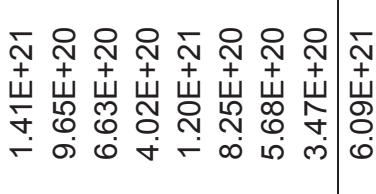 \\
\hline 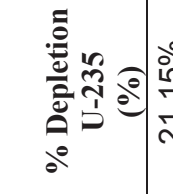 & 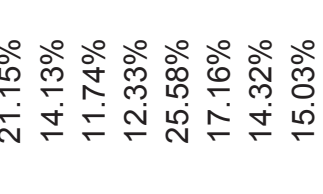 & $m$ & 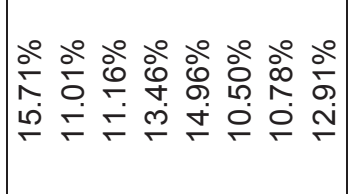 & 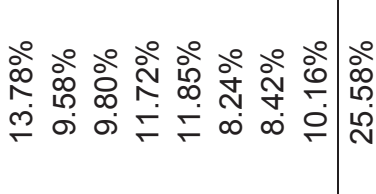 \\
\hline & & & 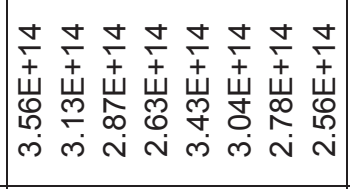 & 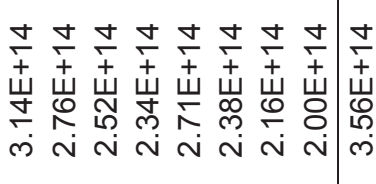 \\
\hline & & 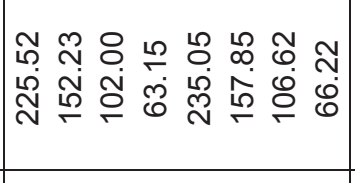 & 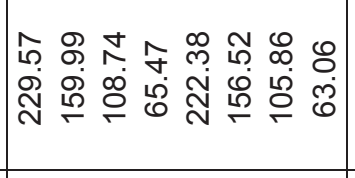 & 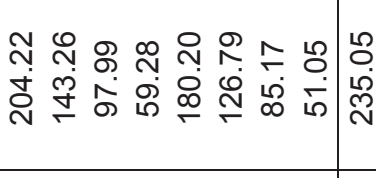 \\
\hline & 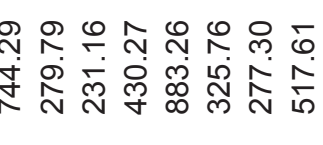 & 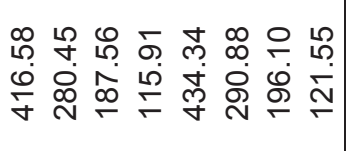 & 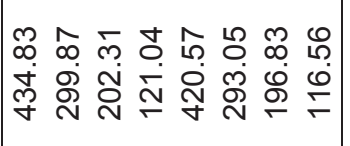 & 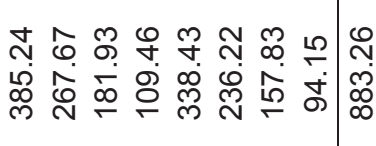 \\
\hline 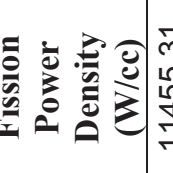 & 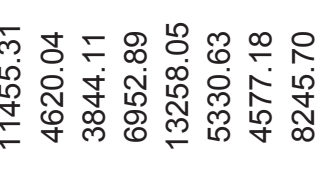 & 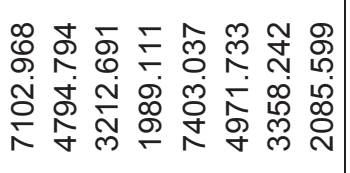 & 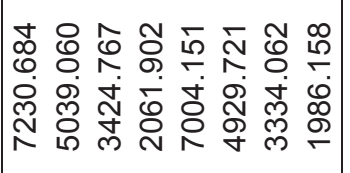 & 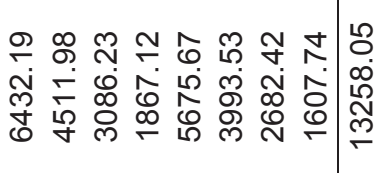 \\
\hline 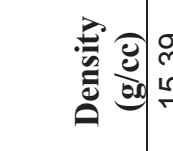 & 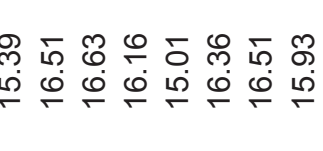 & 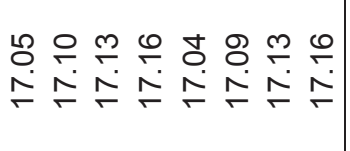 & 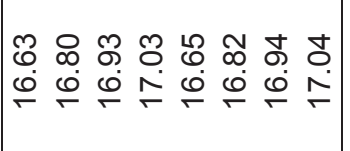 & 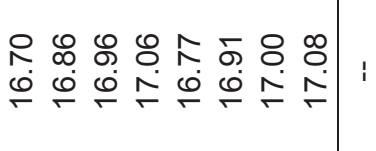 \\
\hline & 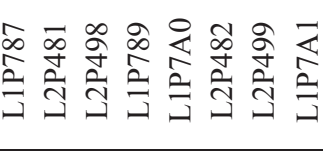 & กิ & 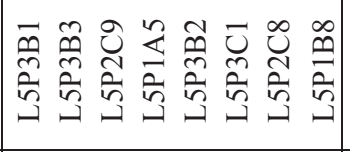 & 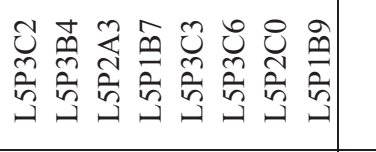 \\
\hline |⿹ & $\varepsilon-z$ & $1-\omega \lambda$ & $\tau-\varepsilon \wedge$ & $\tau-z \lambda$ \\
\hline 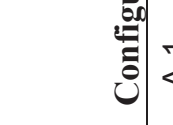 & 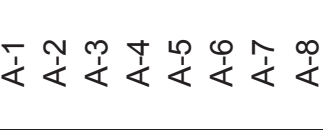 & 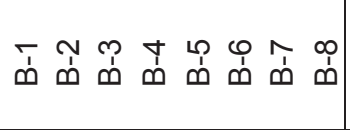 & 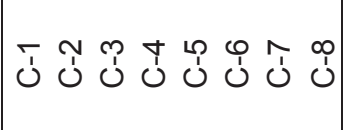 & 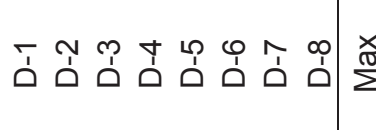 \\
\hline
\end{tabular}




\begin{tabular}{|c|c|c|c|c|}
\hline 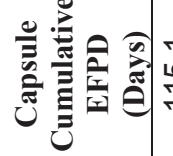 & & 出 & 官 & 象 \\
\hline & 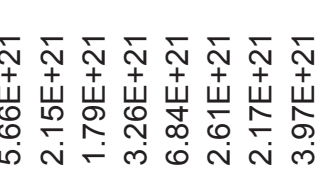 & 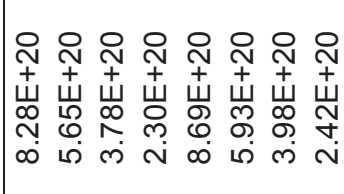 & 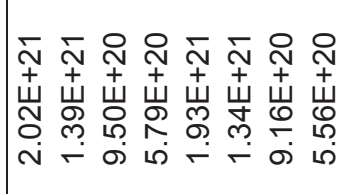 & 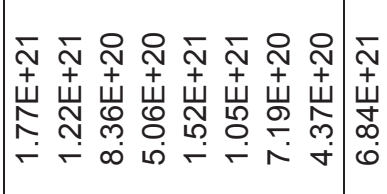 \\
\hline 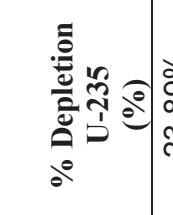 & 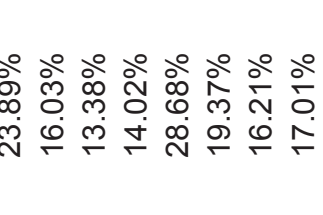 & 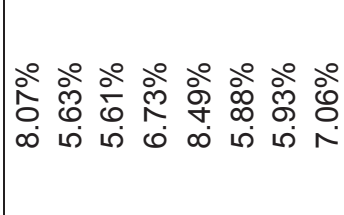 & 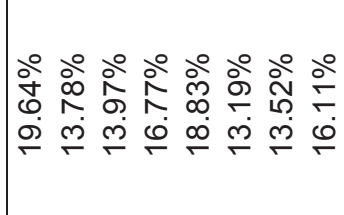 & 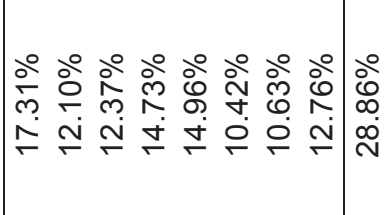 \\
\hline & & & 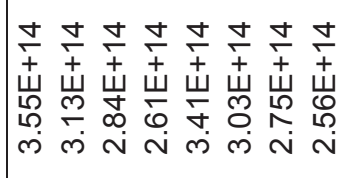 & 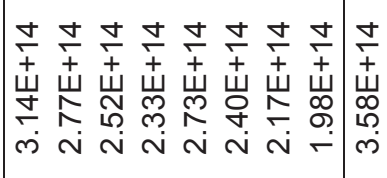 \\
\hline & 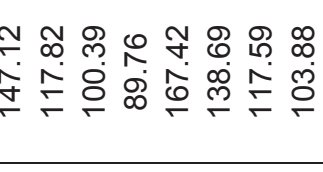 & 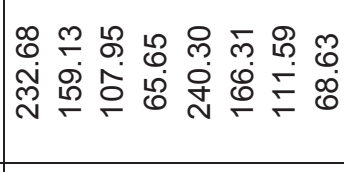 & 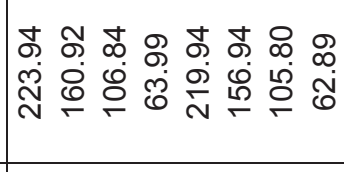 & 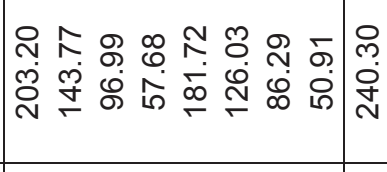 \\
\hline & 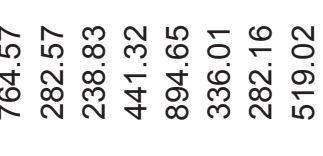 & 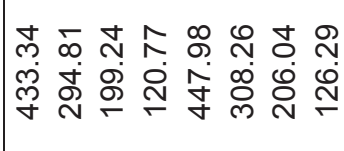 & 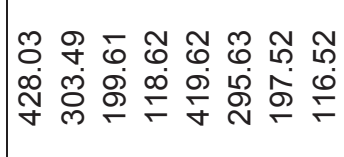 & 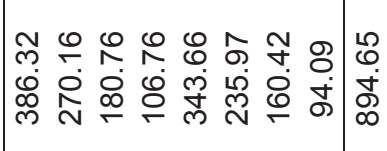 \\
\hline 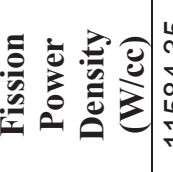 & 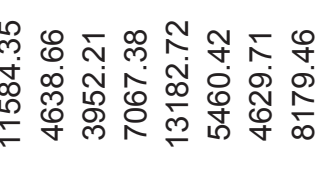 & 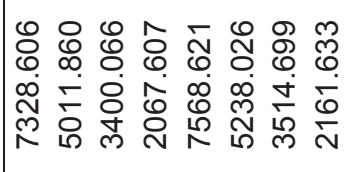 & 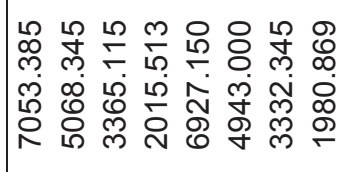 & 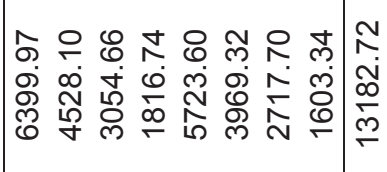 \\
\hline 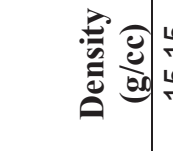 & 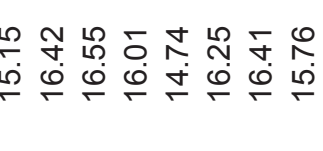 & 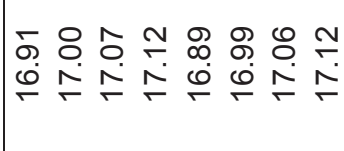 & 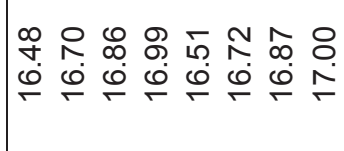 & 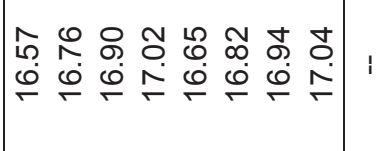 \\
\hline & 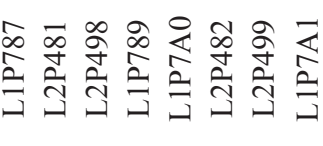 & 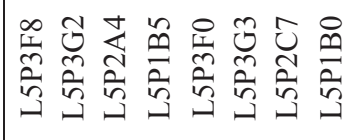 & 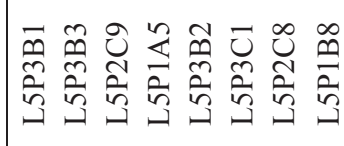 & 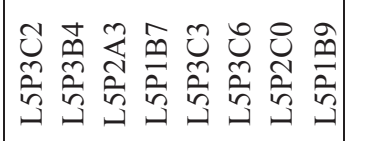 \\
\hline |⿹ & $\varepsilon-z$ & $1-\mu$ & $\tau-\varepsilon \wedge$ & $\tau-\tau \lambda$ \\
\hline : & 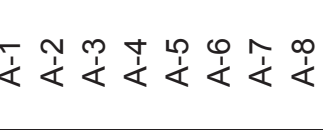 & 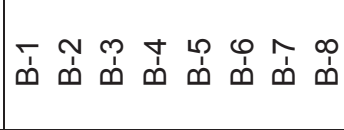 & 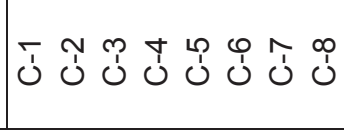 & 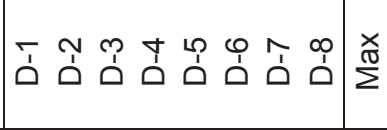 \\
\hline
\end{tabular}




\begin{tabular}{|c|c|c|c|c|}
\hline 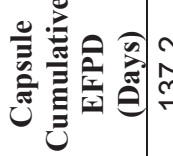 & & i & $\infty$ & $\infty$ \\
\hline & 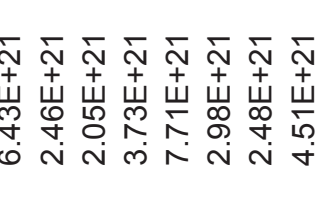 & 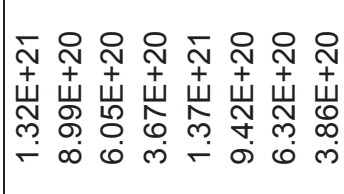 & 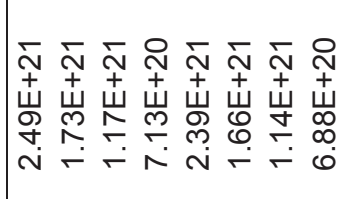 & 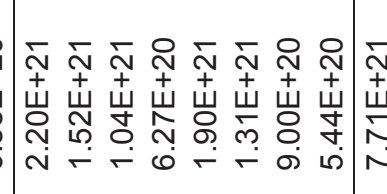 \\
\hline 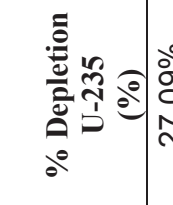 & 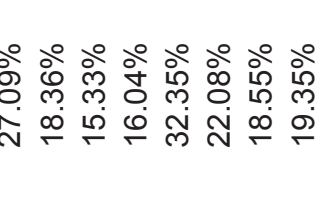 & 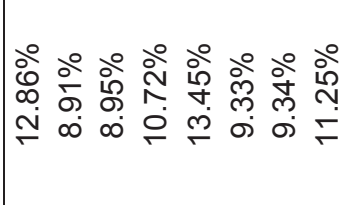 & 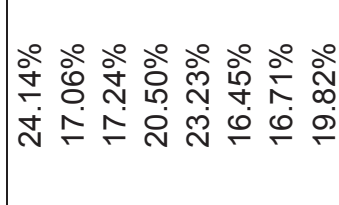 & 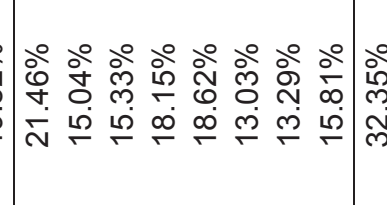 \\
\hline & & & 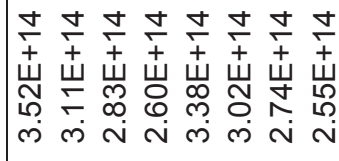 & 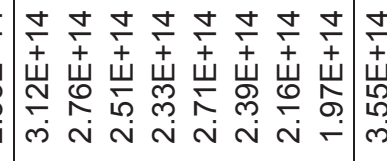 \\
\hline & $\infty_{\infty}^{\infty}$ & 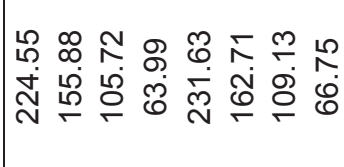 & 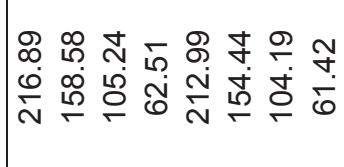 & 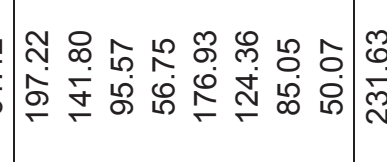 \\
\hline & 象弯 & 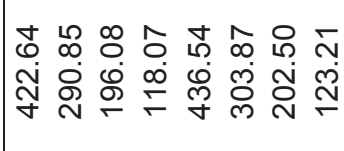 & 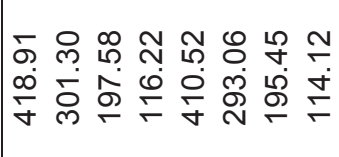 & 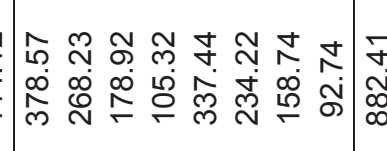 \\
\hline 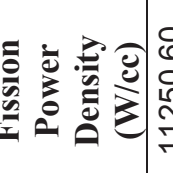 & 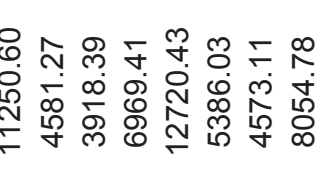 & 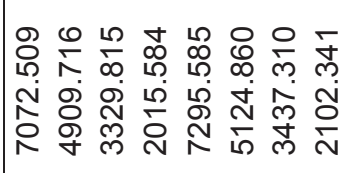 & 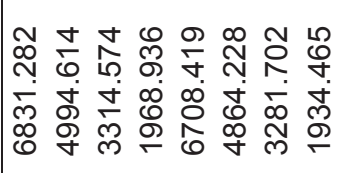 & 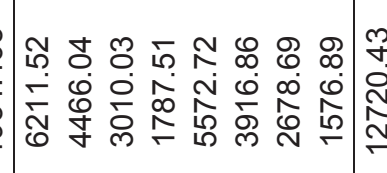 \\
\hline 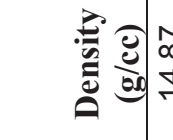 & 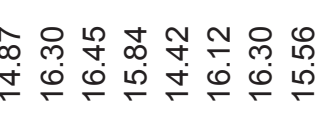 & 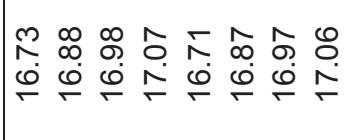 & 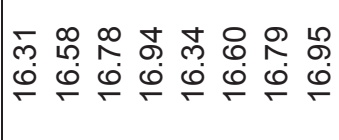 & 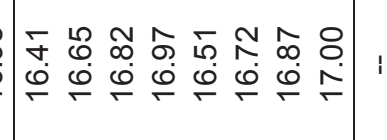 \\
\hline & 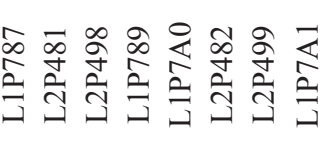 & 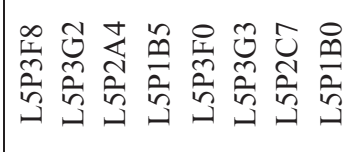 & 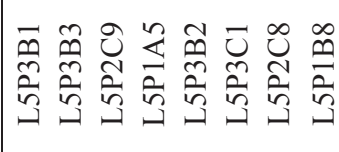 & 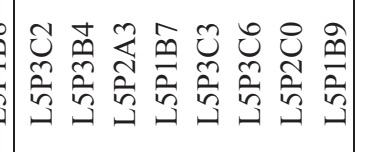 \\
\hline . & $\varepsilon-z$ & $1-\omega$ & $\tau-\varepsilon \curlywedge$ & $\tau-2 \lambda$ \\
\hline 彭。 & 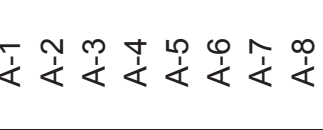 & 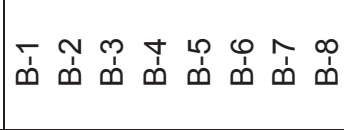 & 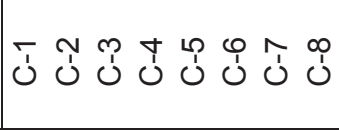 & 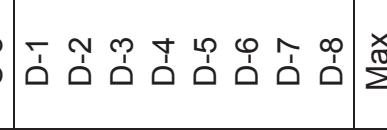 \\
\hline
\end{tabular}




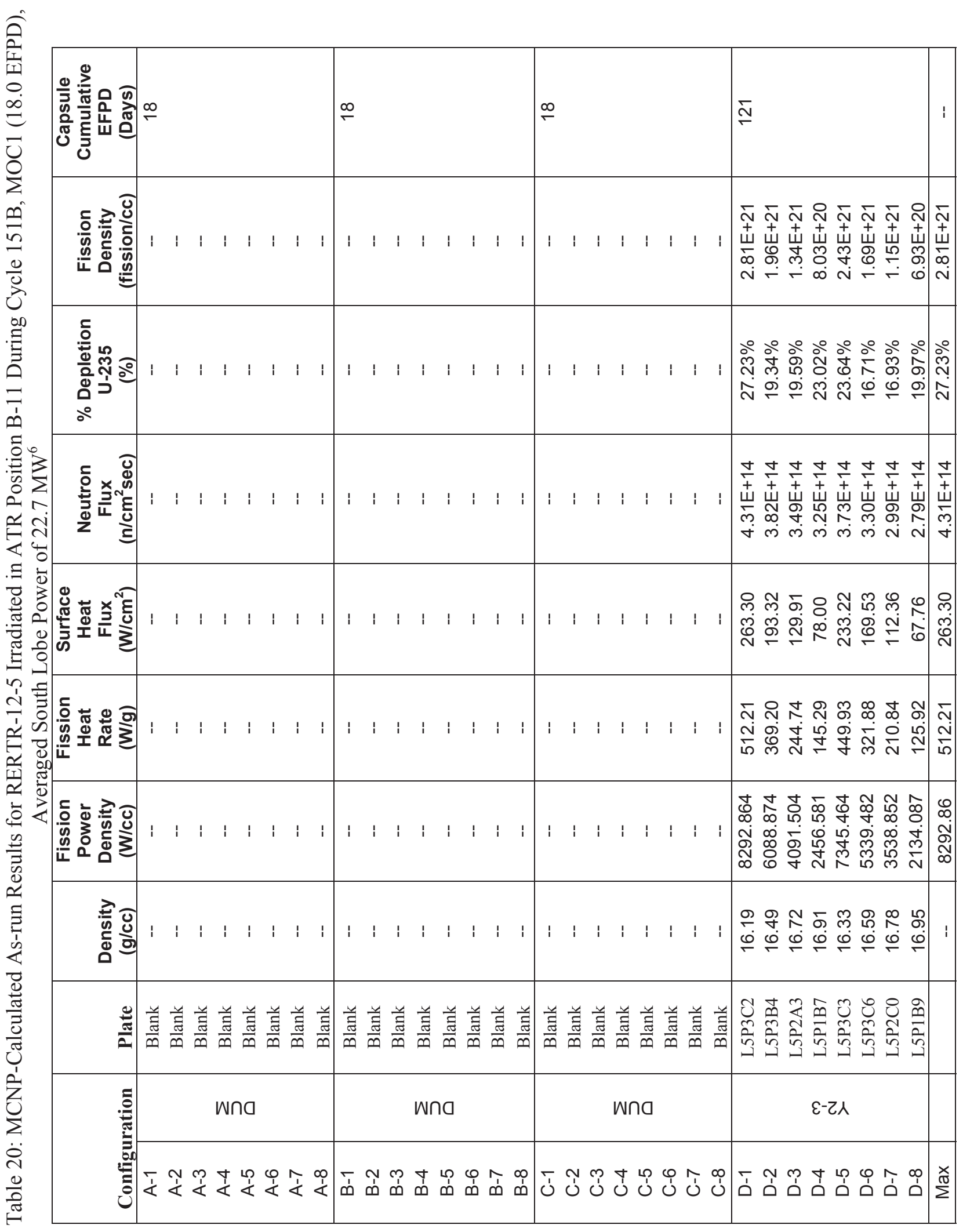




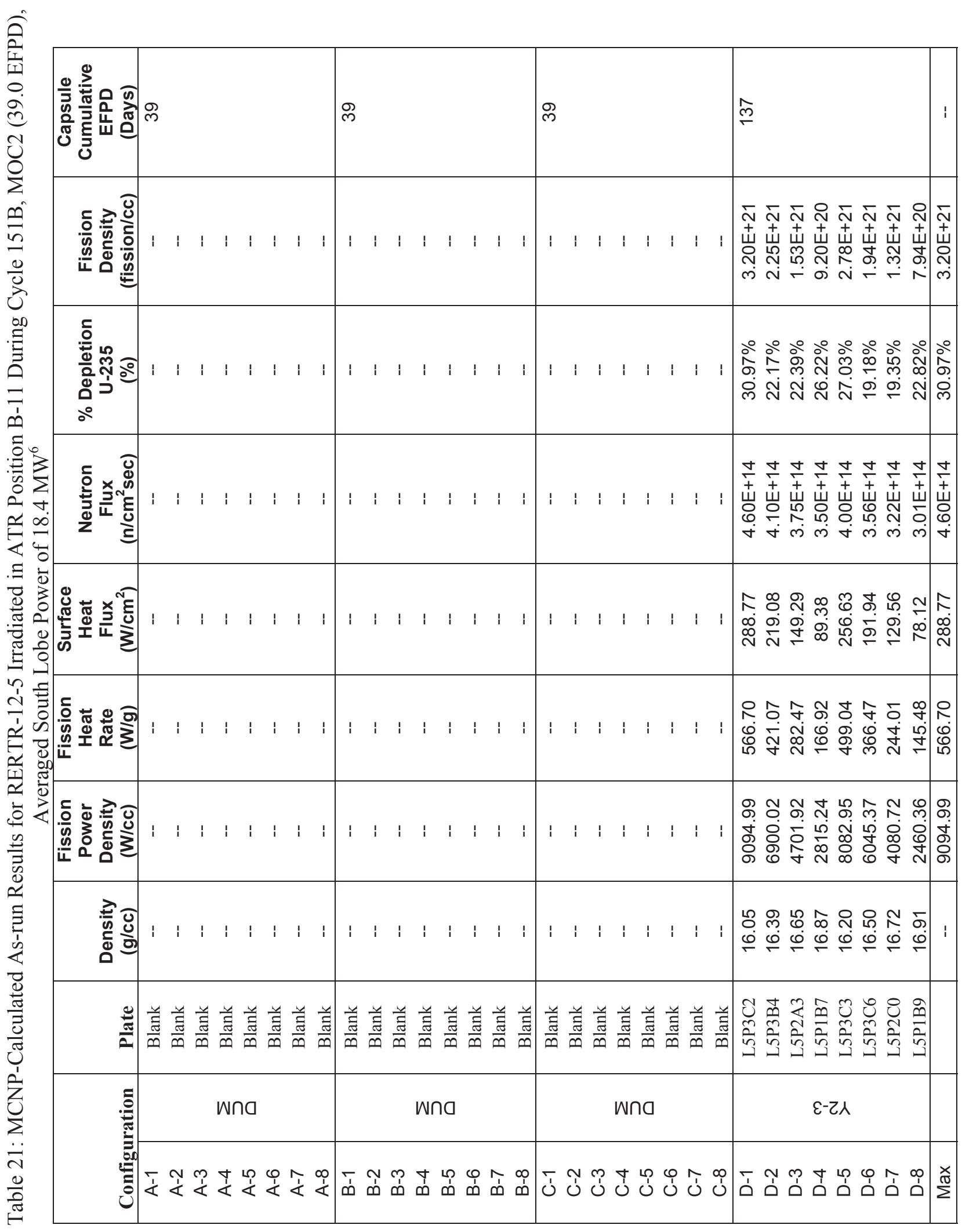




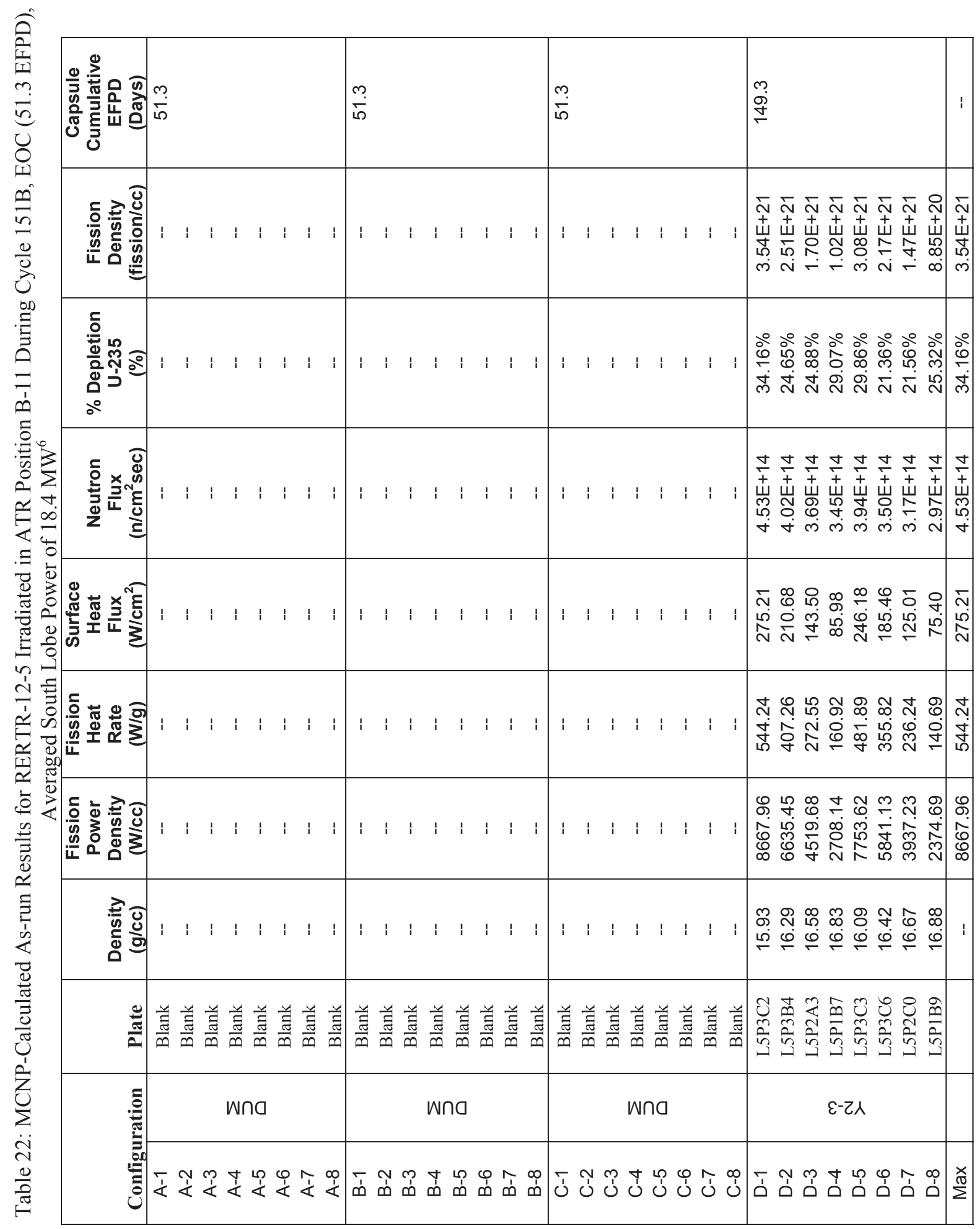




\subsection{Gradients}

The MCNP-calculated power gradients in the transverse and axial directions are represented by the fission rate local-to-average ratios (L2ARs) as a function of position along the fuel foil calculated at the beginning of life. Figure 9 through Figure 12 depict the power gradient in the transverse direction and Figure 13 through Figure 16 depict the power gradient in the axial direction. The 2D gradient map for each plate can be found in Appendix B.

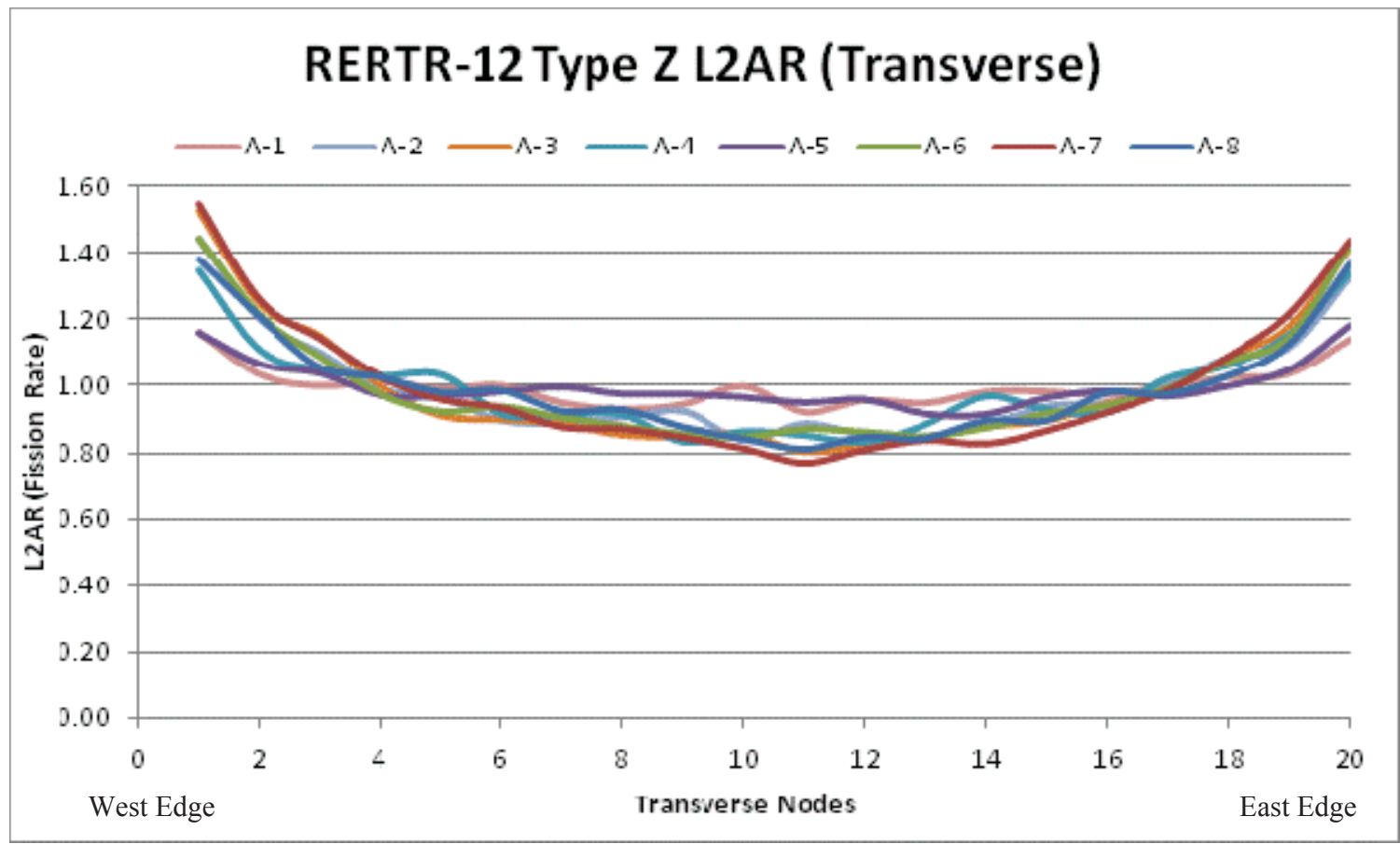

Figure 9: BOL fission rate local to average ratios in the transverse direction for a type $\mathrm{Z}$ capsule in the A capsule position. 


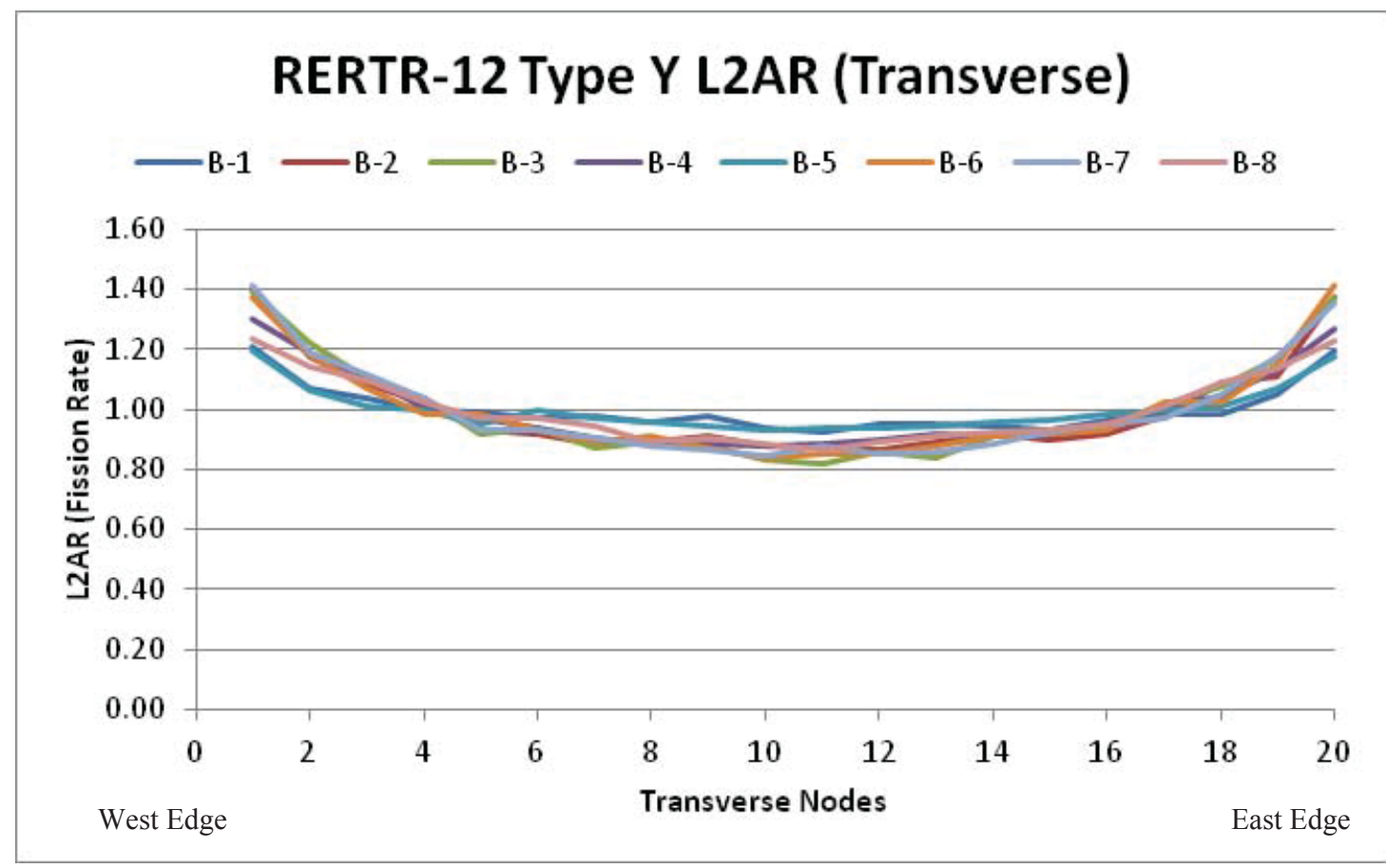

Figure 10: BOL fission rate local to average ratios in the transverse direction for a type $\mathrm{Y}$ capsule in the B capsule position.

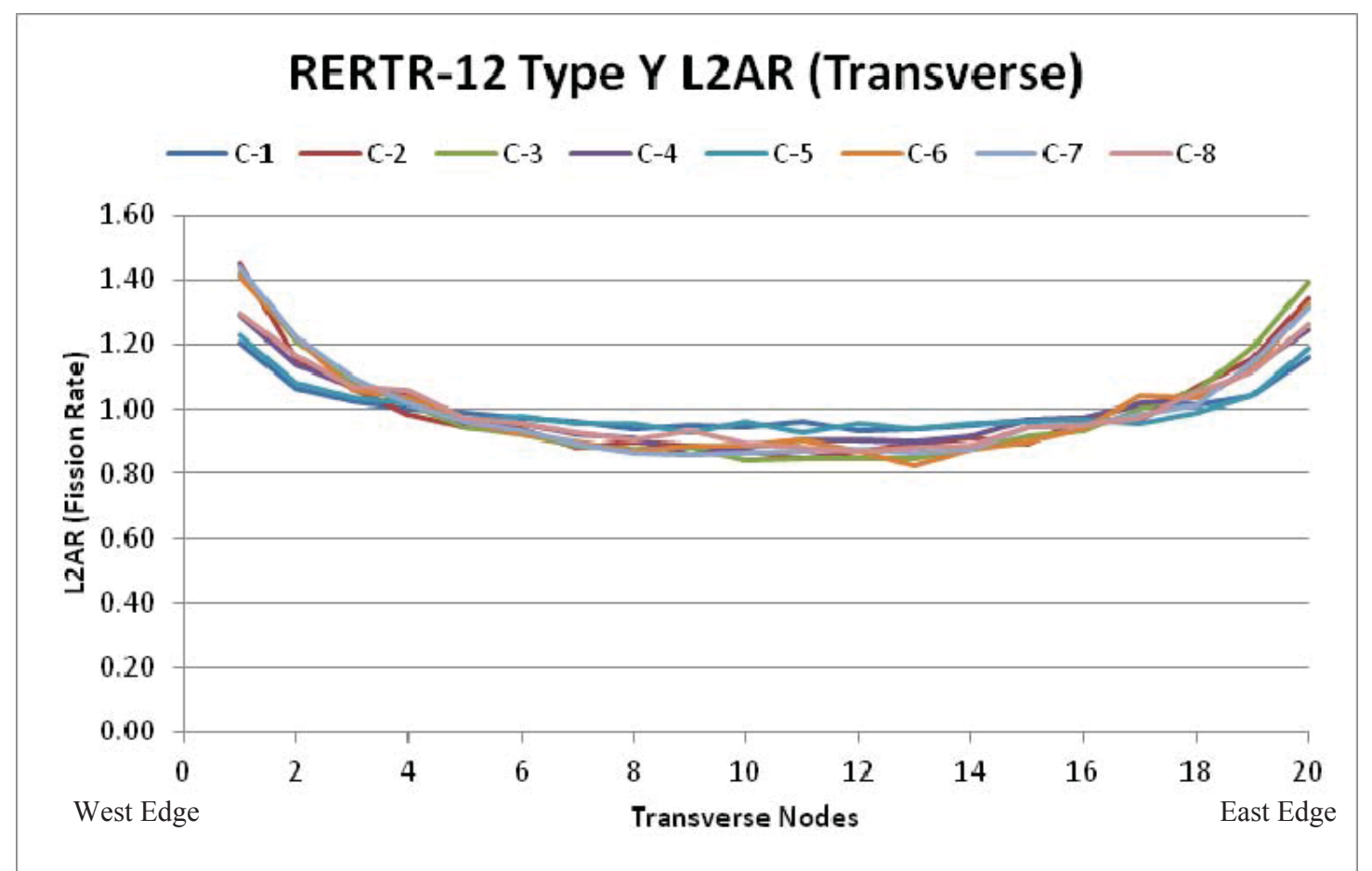

Figure 11: BOL fission rate local to average ratios in the transverse direction for a type $\mathrm{Y}$ capsule in the $\mathrm{C}$ capsule position. 


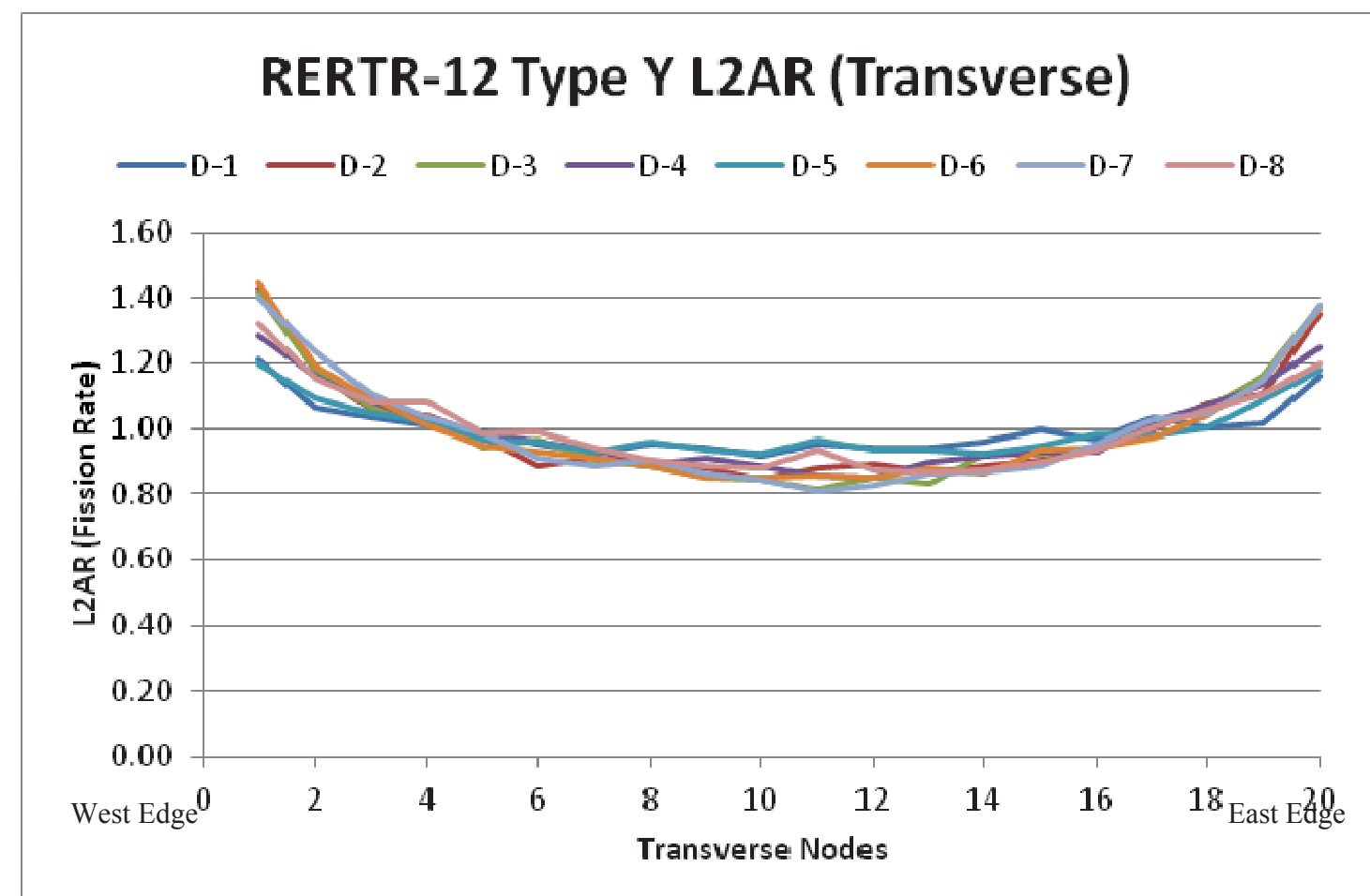

Figure 12: BOL fission rate local to average ratios in the transverse direction for a type Y capsule in the $\mathrm{D}$ capsule position.

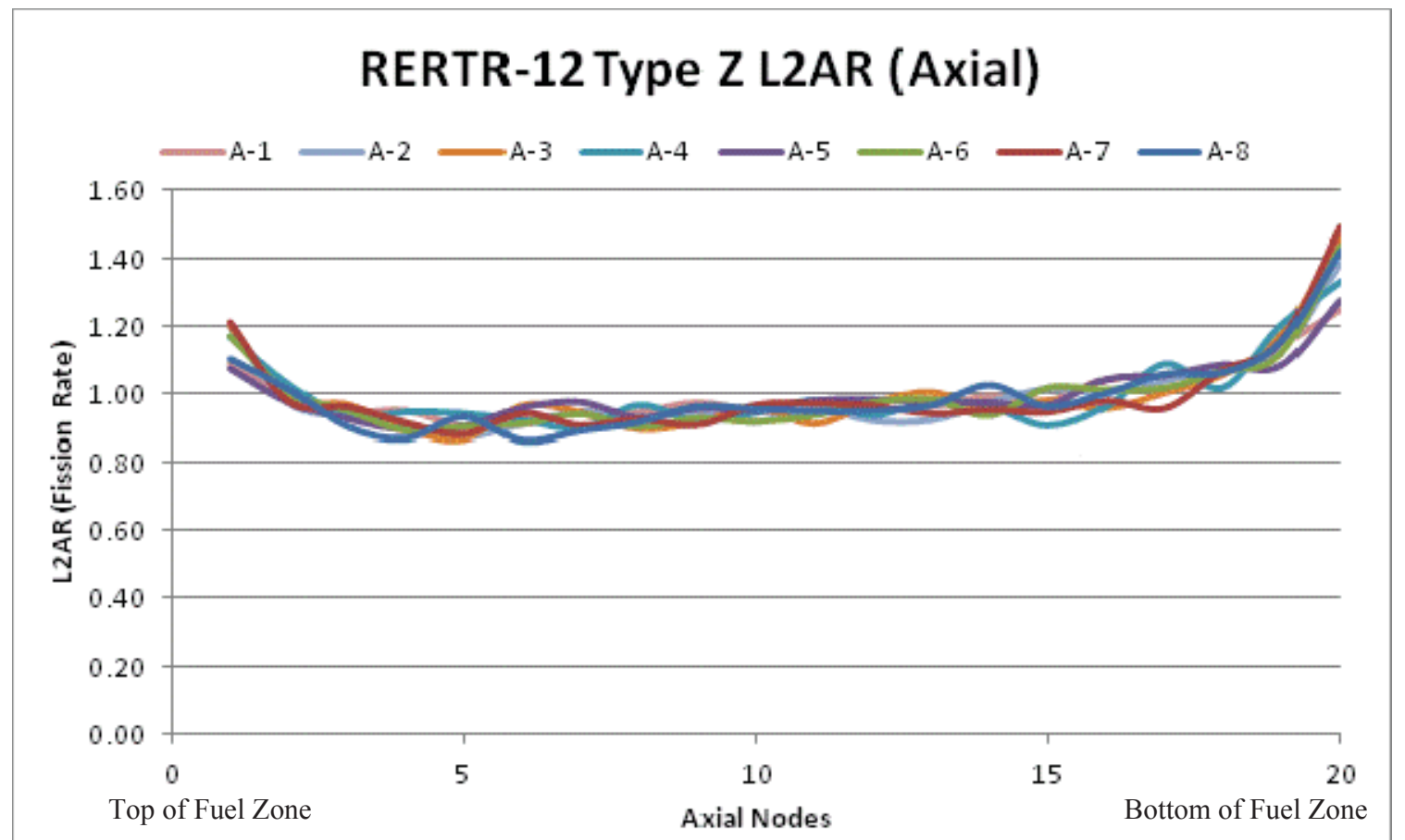

Figure 13: BOL fission rate local to average ratios in the axial direction for a type $\mathrm{Z}$ capsule in the A capsule position. 


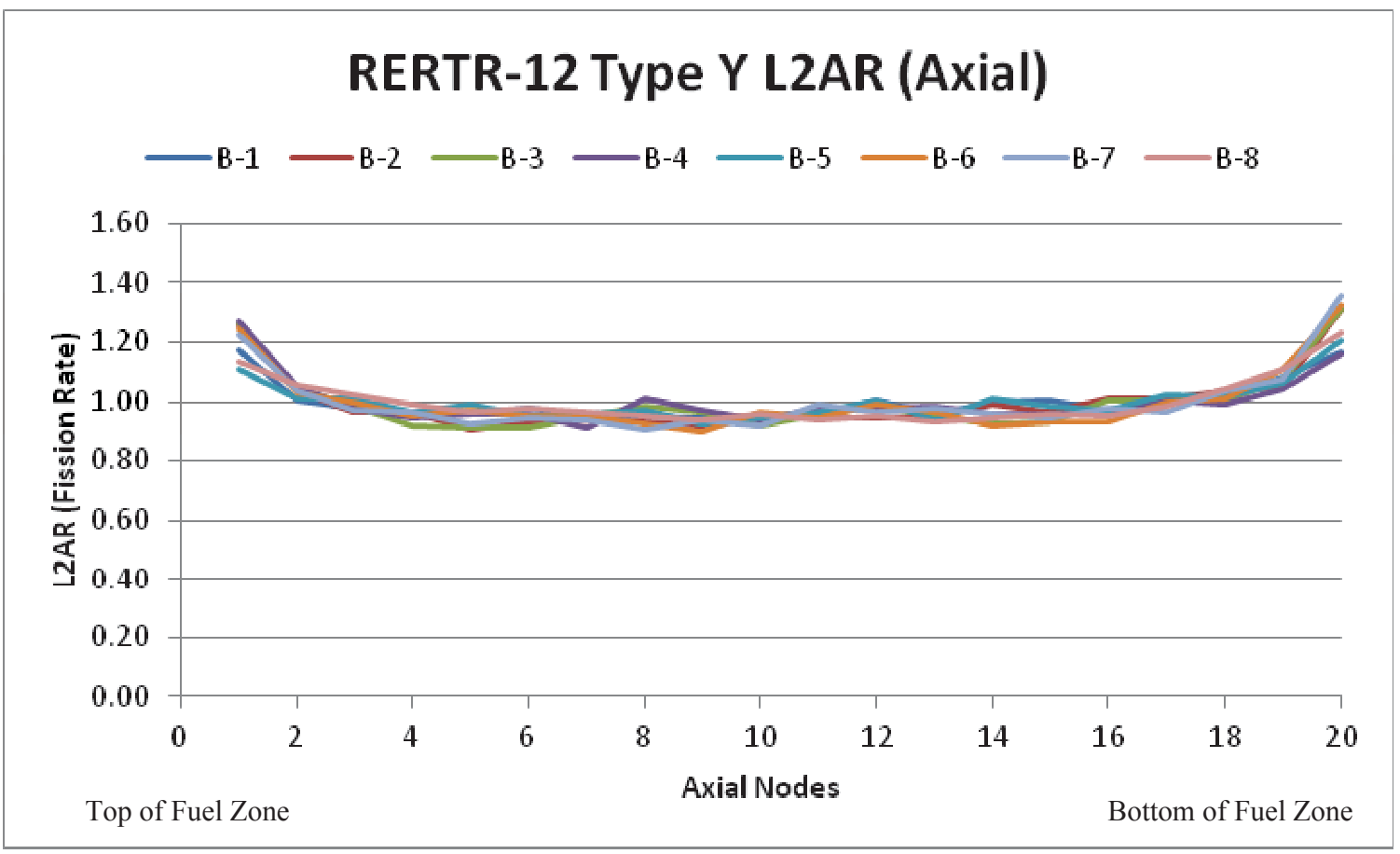

Figure 14: BOL fission rate local to average ratios in the axial direction for a type $\mathrm{Y}$ capsule in the B capsule position.

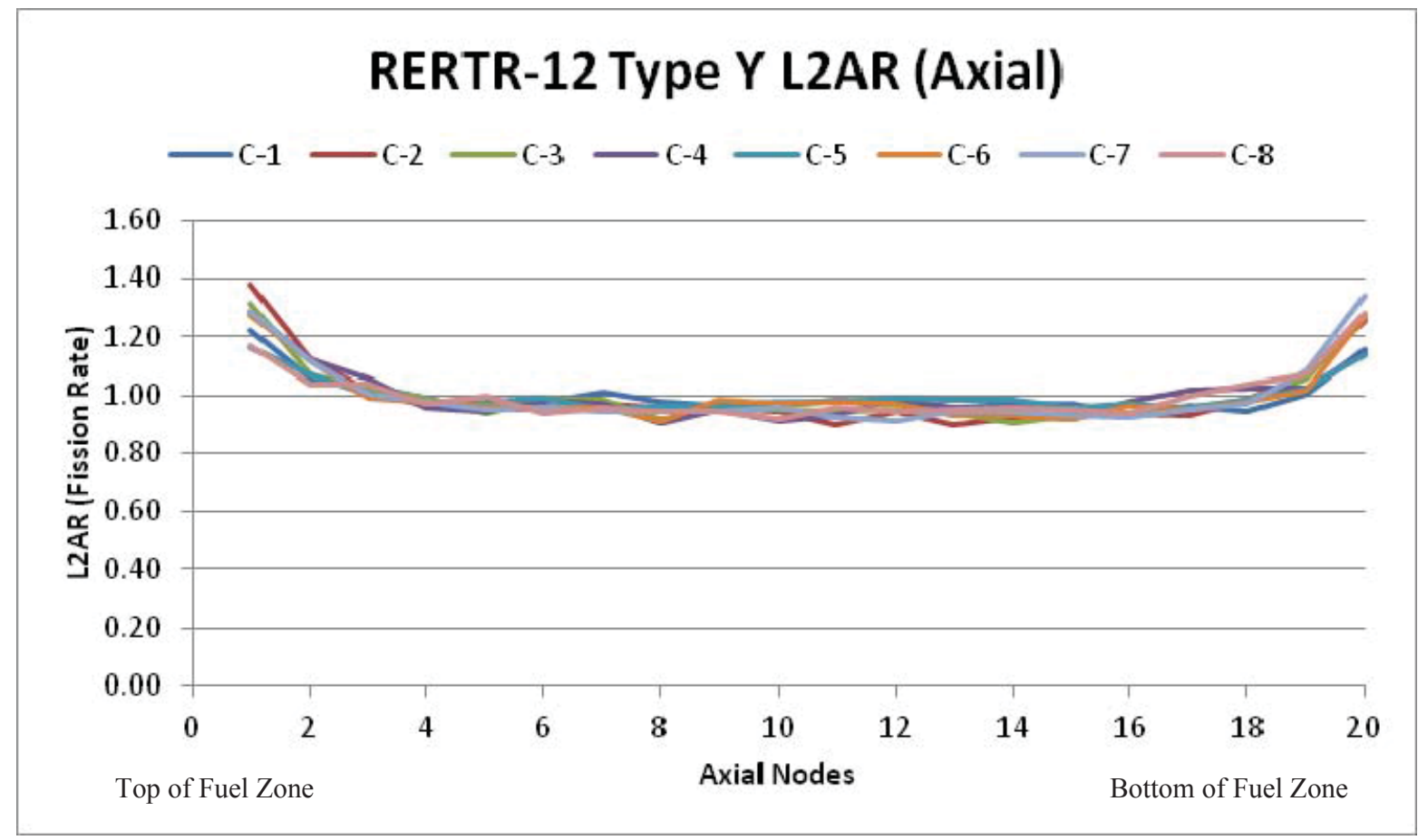

Figure 15: BOL fission rate local to average ratios in the axial direction for a type $\mathrm{Y}$ capsule in the $\mathrm{C}$ capsule position. 


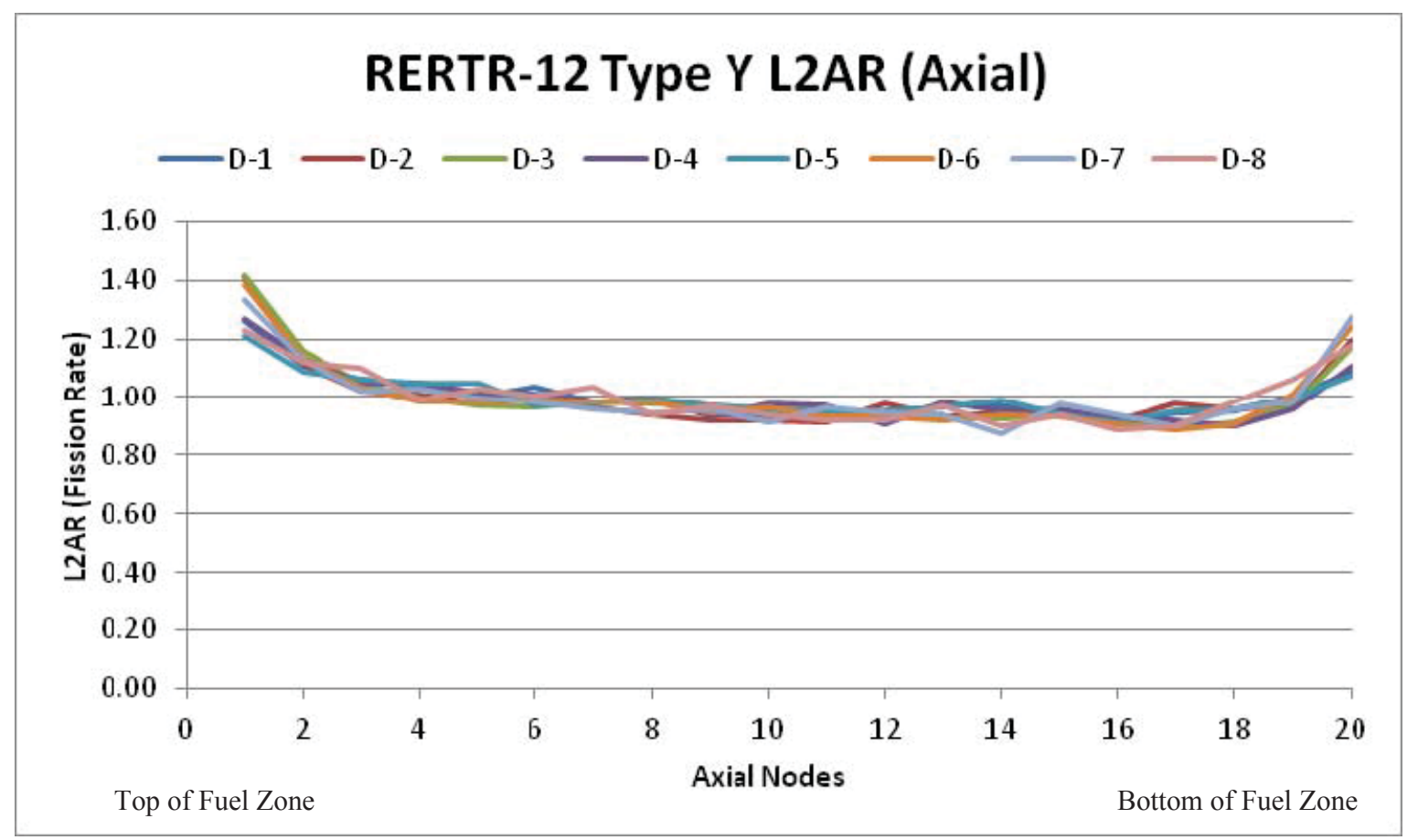

Figure 16: BOL fission rate local to average ratios in the axial direction for a type $\mathrm{Y}$ capsule in the $\mathrm{D}$ capsule position. 


\section{HYDRAULIC TESTING}

A fully assembled irradiation test vehicle (with simulated fuel plates) was used for testing. The test vehicle was fabricated such that the orifice plates could be easily changed. The hydraulic resistance of the RERTR Large B-Position irradiation test vehicle with various orifice plate sizes were calculated, the results are shown in Table 23.

Table 23: Loss Coefficients for the RERTR Irradiation Test Vehicle Components ${ }^{7}$

\begin{tabular}{ccc}
\hline $\begin{array}{c}\text { Orifice Dia. } \\
(\mathrm{mm})\end{array}$ & $\begin{array}{c}\mathrm{K} / \mathrm{A}^{2} \\
\left(1 / \mathrm{m}^{4}\right)\end{array}$ & $\begin{array}{c}\text { ATR Coolant } \\
\text { Flow Rate } \\
\left(\mathrm{cm}^{3} / \mathrm{sec}\right)\end{array}$ \\
\hline 10 & $5.3041 \times 10^{8}$ & 1252 \\
\hline 9 & $8.2181 \times 10^{8}$ & 1046 \\
\hline 8 & $1.6961 \times 10^{9}$ & 757 \\
\hline 7.32 & $2.9022 \times 10^{9}$ & 588 \\
\hline 7 & $3.0058 \times 10^{9}$ & 579 \\
\hline 6 & $4.0784 \times 10^{9}$ & 500 \\
\hline 5 & $101743 \times 10^{10}$ & 298 \\
\hline Bypass & $2.7958 \times 10^{8}$ & -- \\
Vehicle & $1.4161 \times 10^{8}$ & 2727 \\
\hline
\end{tabular}

Based on the results from the hydraulic testing, the orifice was removed leaving the capsule in the "Vehicle" configuration to provide an ATR coolant flow rate through the capsules of $2727 \mathrm{~cm}^{3} / \mathrm{sec}^{8}$ 


\section{AS-RUN THERMAL ANALYSIS}

The thermal as-run analysis was performed using the as-built geometry, MCNP-calculated surface heat flux $\left(\mathrm{W} / \mathrm{cm}^{2}\right)$ and nominal coolant channel flow rate. ABAQUS ${ }^{9}$ was used to calculate the coolant channel temperatures and plate surface temperatures.

The heat transfer correlation used to calculate these temperatures was calculated from the Colburn equation (equation 5-50c from Reference 10):

$$
N u=\frac{h D}{k}=0.023 R e^{0.8} \operatorname{Pr}^{0.3}
$$

Where $\mathrm{Nu}$ is the Nusselt number, $\mathrm{h}$ is the heat transfer coefficient, $\mathrm{D}$ is the hydraulic diameter, $\mathrm{k}$ is the thermal conductivity, Re is the Reynolds number and Pr is the Prandlt number.

The thermal analysis was performed using the beginning of life L2ARs shown in Section 5.2.

\subsection{Coolant Channel Temperature}

The coolant temperature was analyzed at the five flow channels in the capsule (see Figure 17). For each interval, the coolant temperature was plotted as a function of location along the test assembly with 0.0 in. being at the top of the assembly. These plots are shown in Figure 18 through Figure 29.

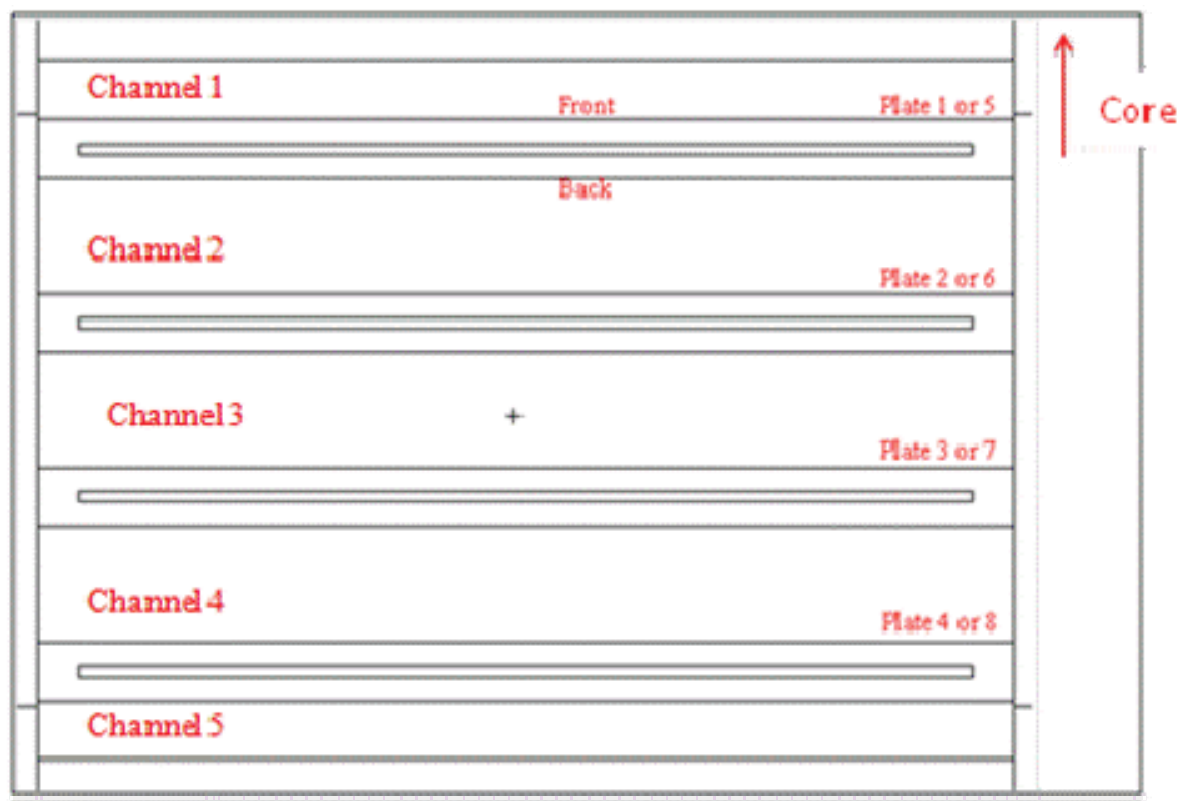

Figure 17: RERTR-12 capsule cross section with the front (side with plate ID) of plate 1 facing the core. 


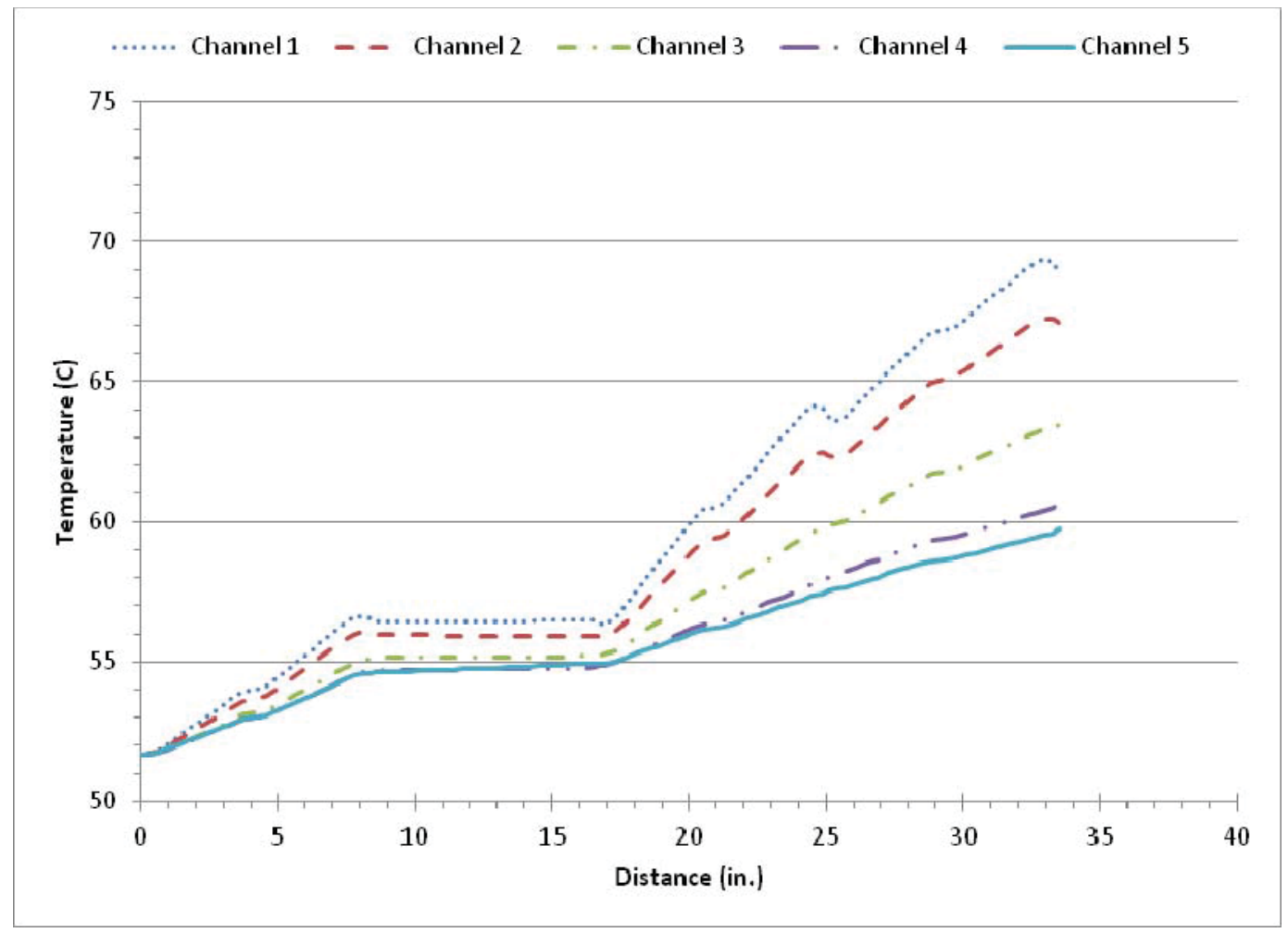

Figure 18: Coolant channel temperatures as a function of location along the RERTR-12 test assembly at BOC 150B (0.0 EFPD).

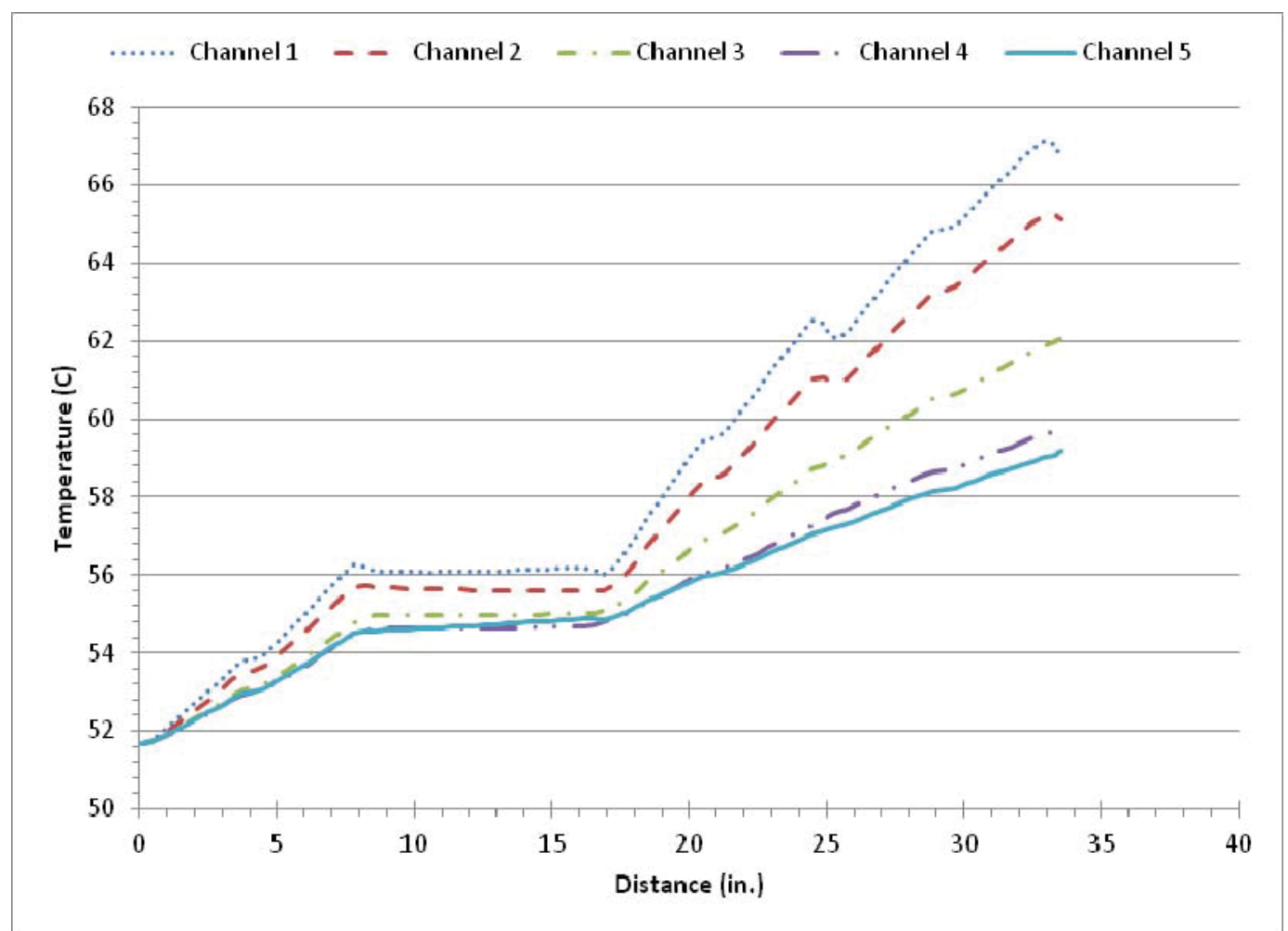

Figure 19: Coolant channel temperature as a function of location along the RERTR-12 test assembly at MOC1 150B (18.0 EFPD). 


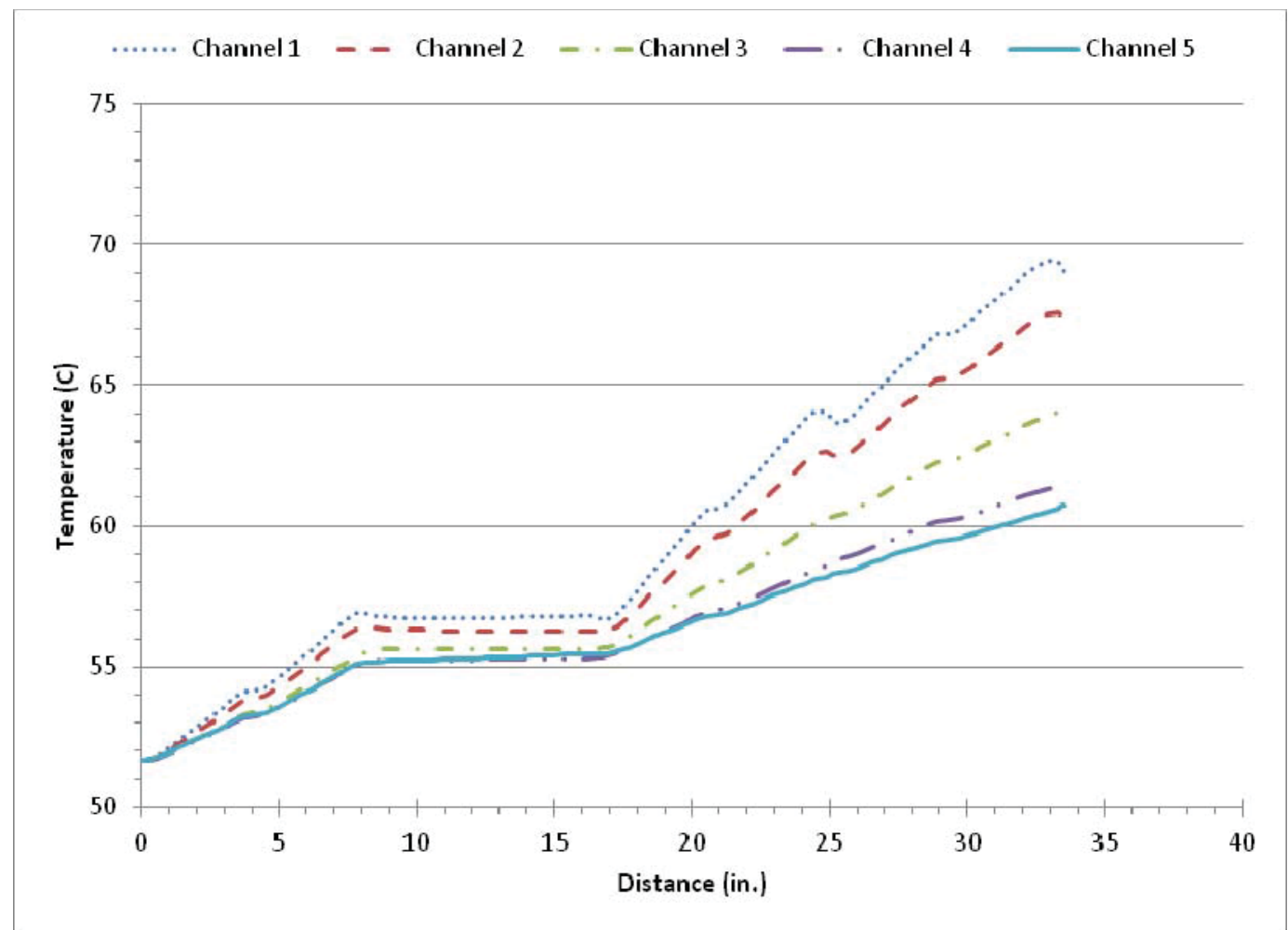

Figure 20: Coolant channel temperature as a function of location along the RERTR-12 test assembly at MOC2 150B (31.0 EFPD).

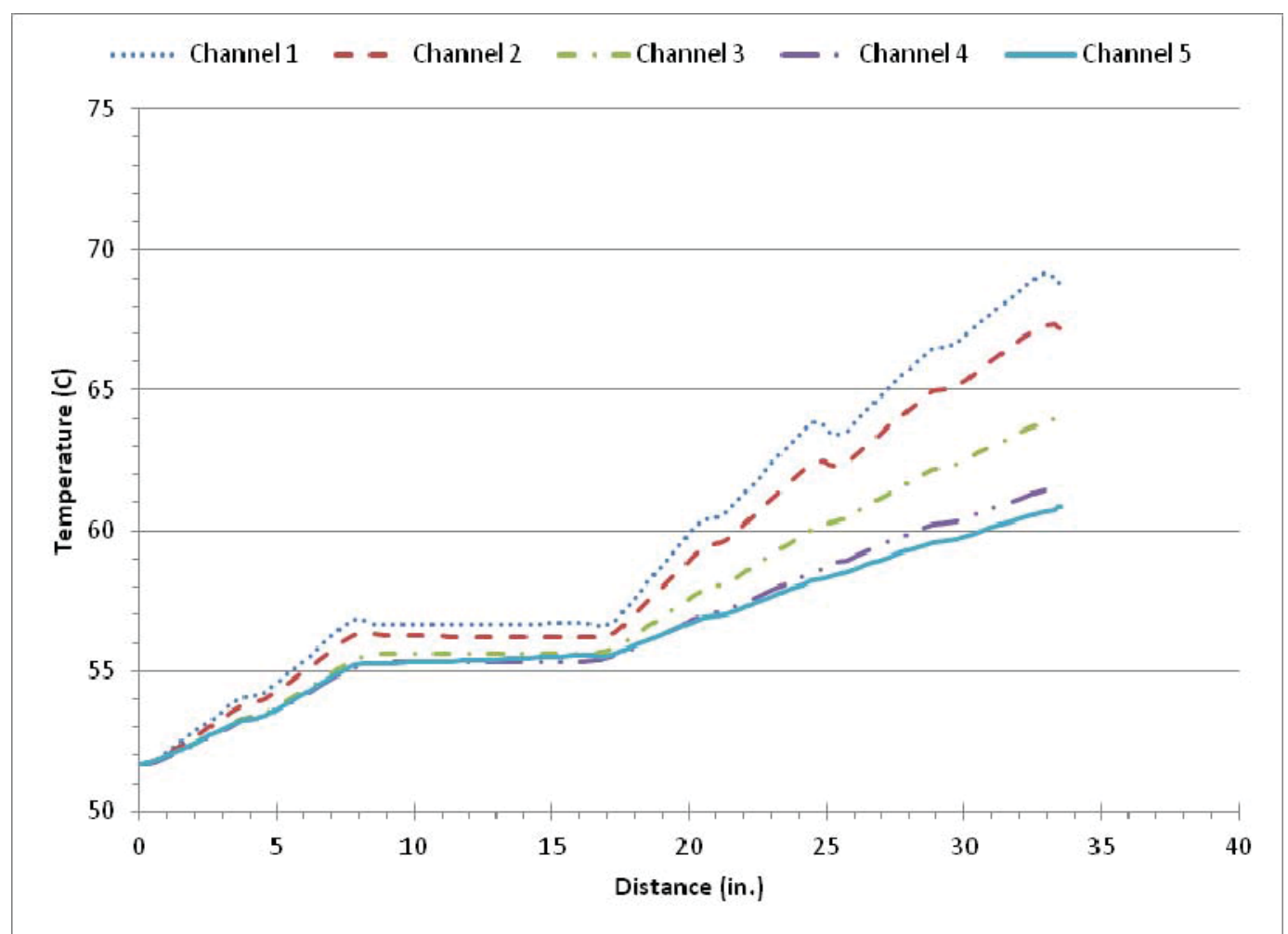

Figure 21: Coolant channel temperature as a function of location along the RERTR-12 test assembly at EOC 150B (41.9 EFPD). 


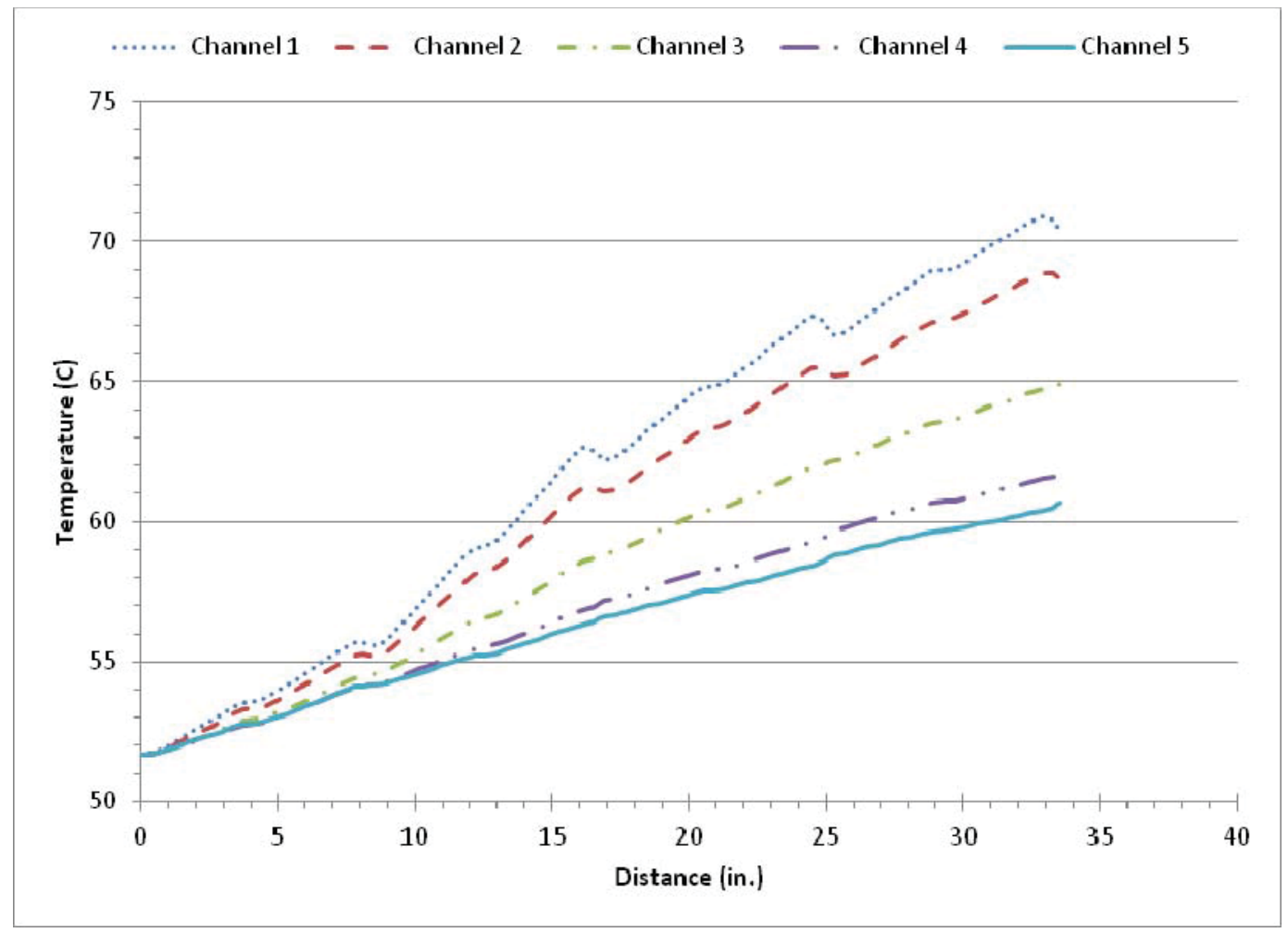

Figure 22: Coolant channel temperatures as a function of location along the RERTR-12 test assembly at BOC 151A (0.0 EFPD).

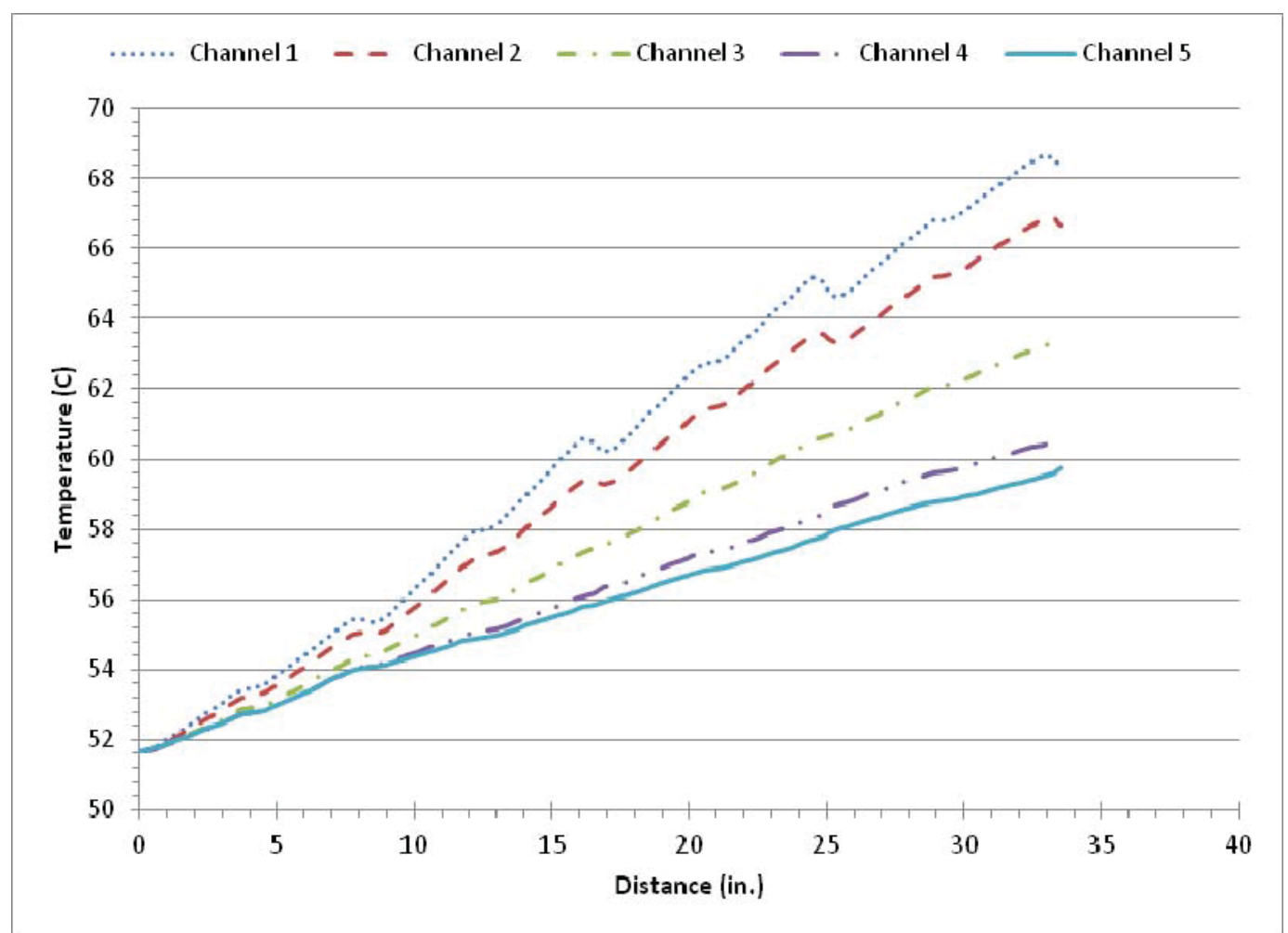

Figure 23: Coolant channel temperature as a function of location along the RERTR-12 test assembly at MOC1 151A (15.0 EFPD). 


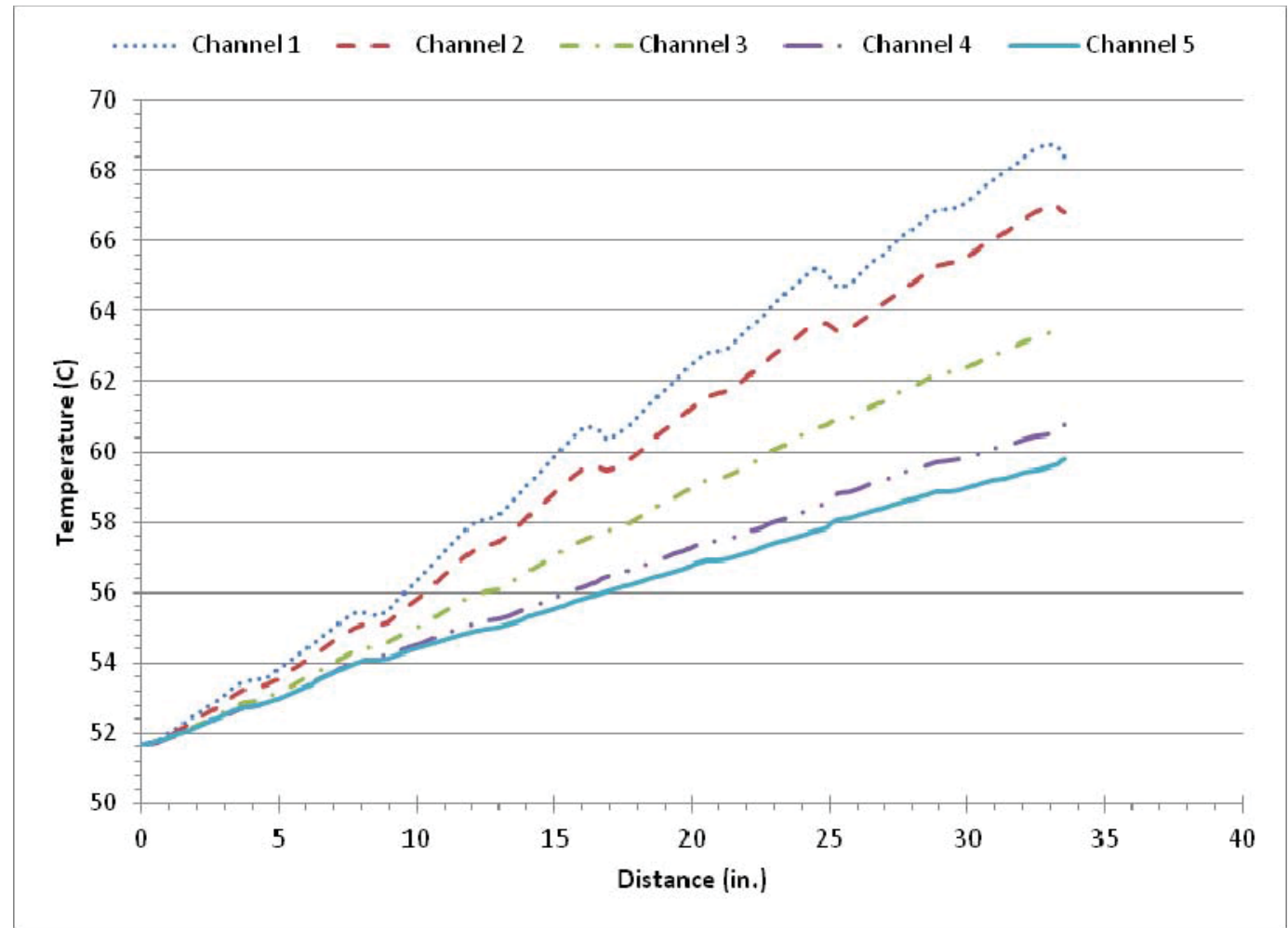

Figure 24: Coolant channel temperature as a function of location along the RERTR-12 test assembly at MOC2 151A (34.0 EFPD).

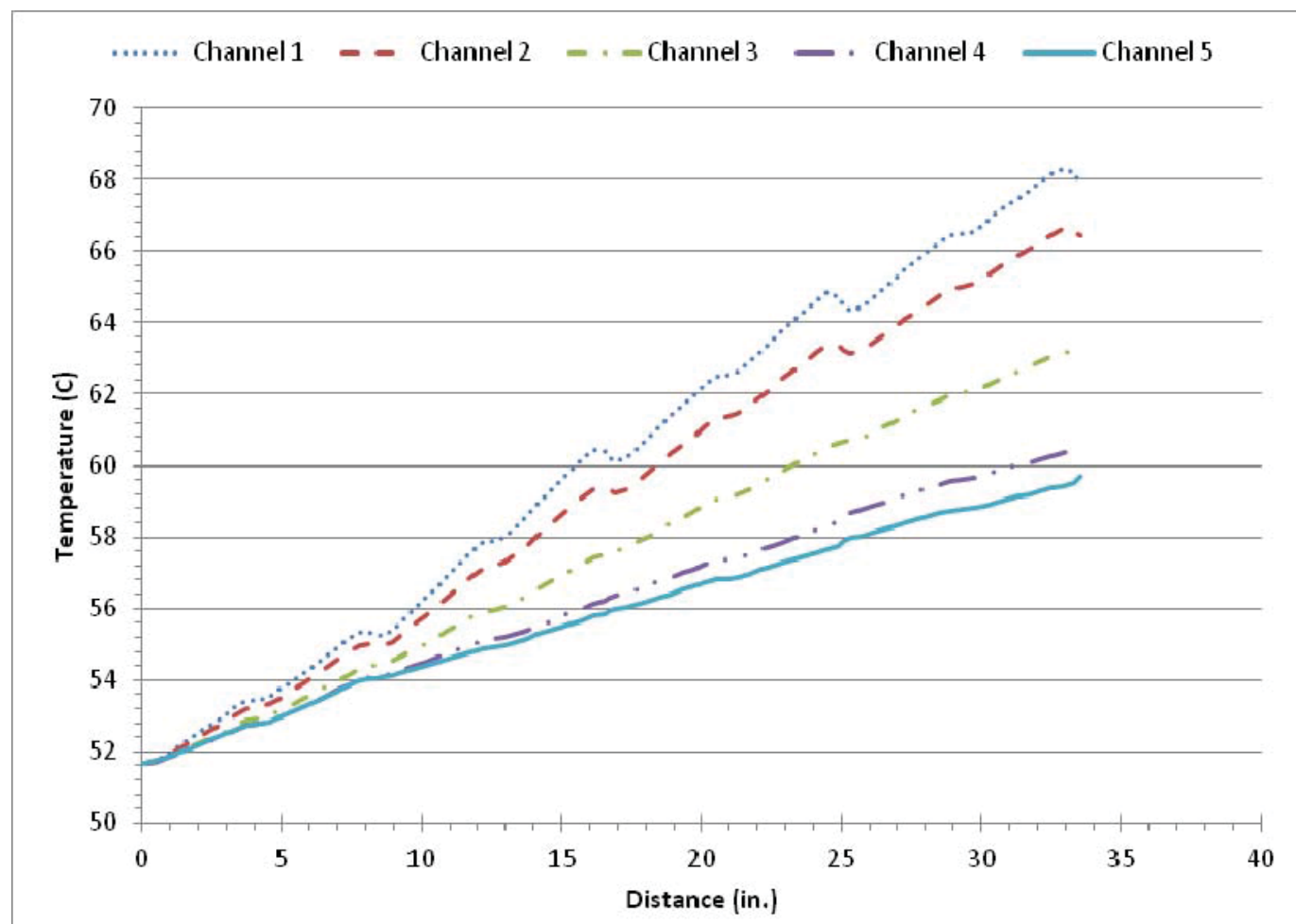

Figure 25: Coolant channel temperature as a function of location along the RERTR-12 test assembly at EOC 151A (56.1 EFPD). 


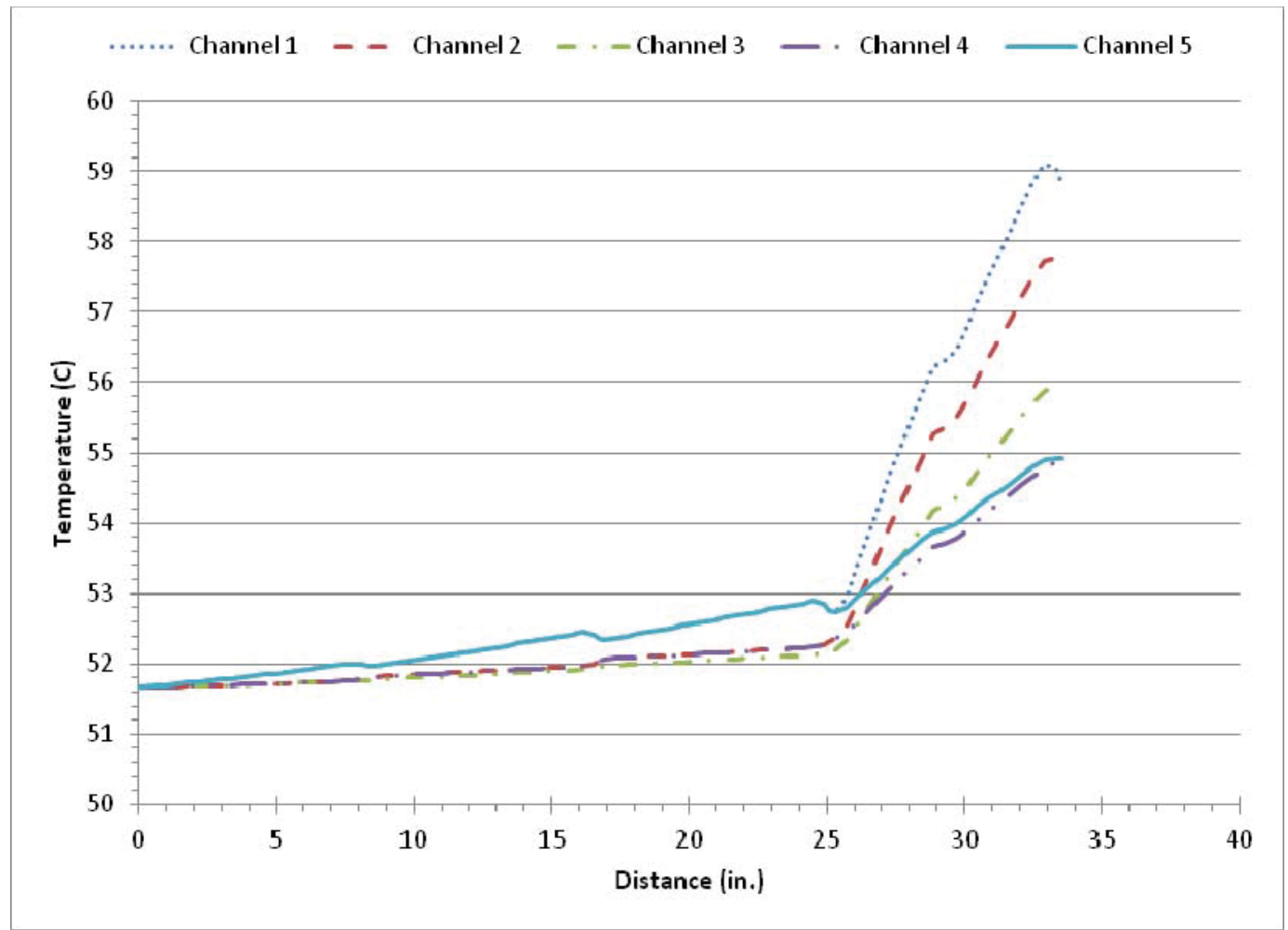

Figure 26: Coolant channel temperature as a function of location along the RERTR-12 test assembly at BOC 151B (0.0 EFPD).

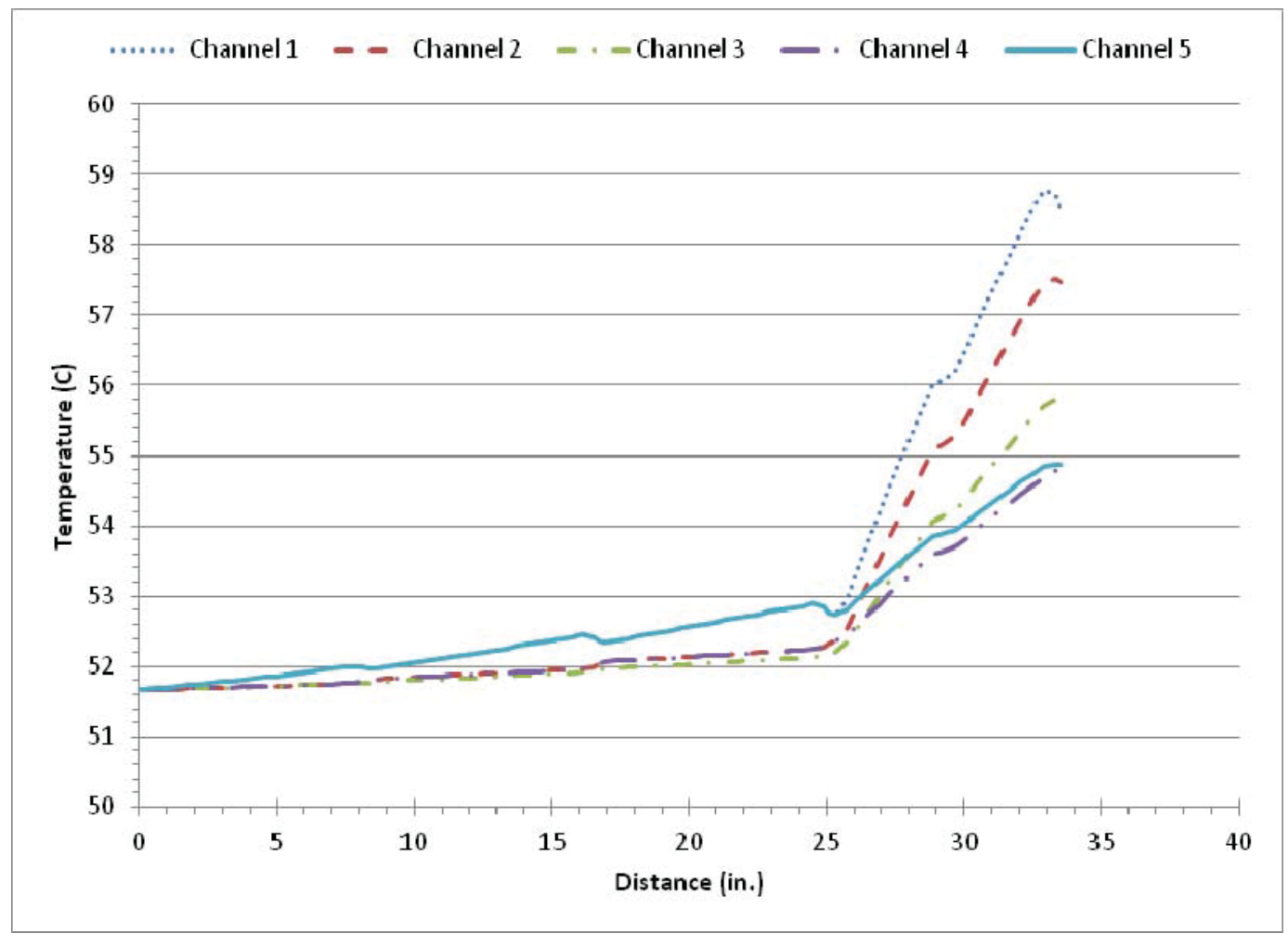

Figure 27: Coolant channel temperature as a function of location along the RERTR-12 test assembly at MOC1 151B (23.0 EFPD). 


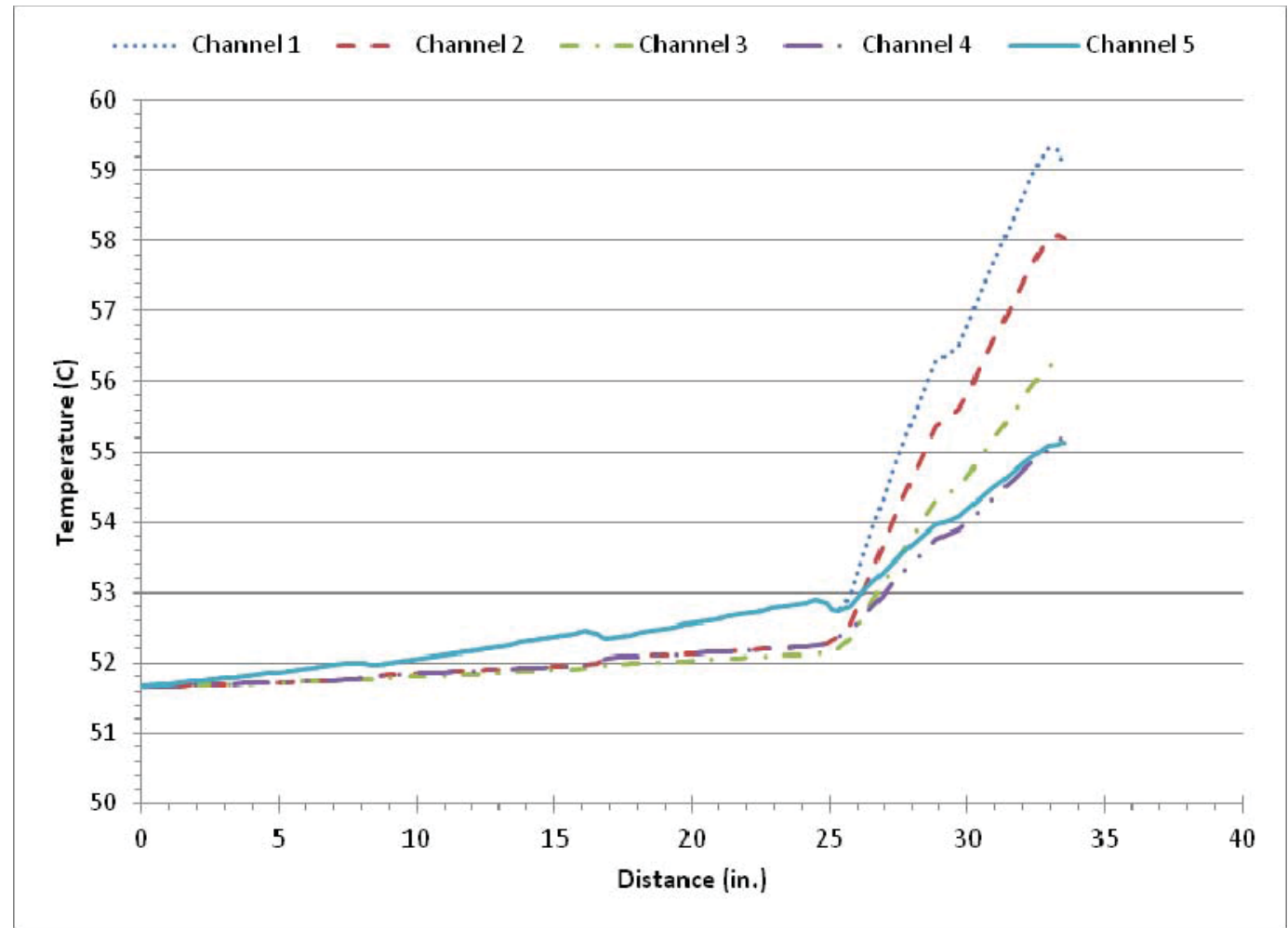

Figure 28: Coolant channel temperature as a function of location along the RERTR-12 test assembly at MOC2 151B (39.0 EFPD).

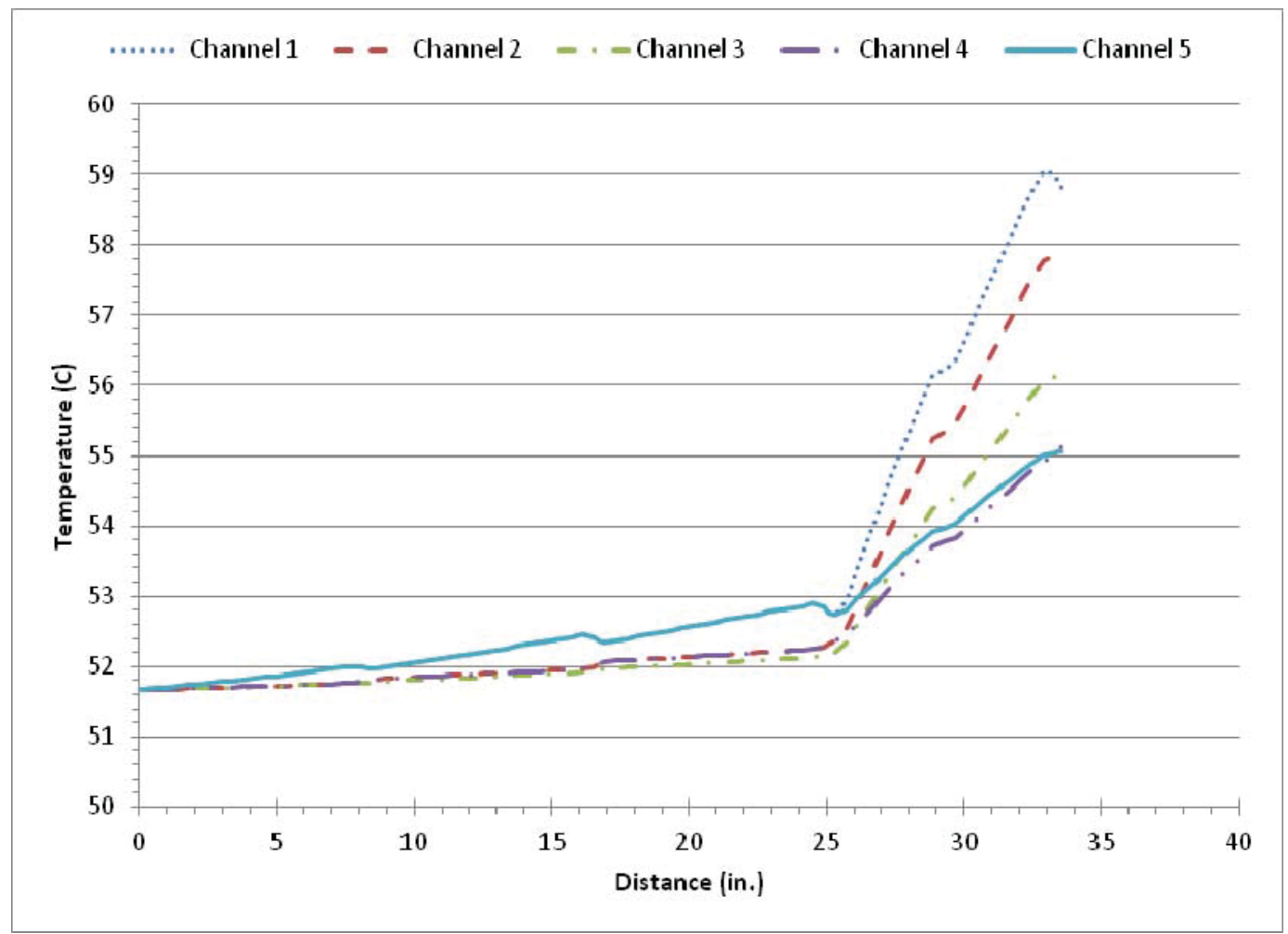

Figure 29: Coolant channel temperature as a function of location along the RERTR-12 test assembly at EOC 151B (51.3 EFPD). 


\subsection{Plate Surface Temperature}

The minimum, maximum and average plate surface temperatures over the fuel zone on each side of the plate are shown in Table 24 through Table 47, where the front of the plate (plate ID side) is facing the core $^{11}$, for cycle $150 \mathrm{~B}$ and $151 \mathrm{~A}$ the South side of the plate is facing core, for cycle 151B, the North side of the plate is facing core (see Figure 17).

Table 24: As-run minimum, maximum and average plate surface temperatures over fuel zone on the south side of the plate for plates irradiated in Cycle 150B, BOC (0 EFPD)

\begin{tabular}{|c|c|c|c|c|}
\hline $\begin{array}{c}\text { Plate } \\
\text { Location }\end{array}$ & Plate ID & $\begin{array}{c}\text { Minimum } \\
\text { Temperature }\end{array}$ & $\begin{array}{c}\text { Maximum } \\
\text { Temperature } \\
(\mathrm{C})\end{array}$ & $\begin{array}{c}\text { Average } \\
\text { Temperature } \\
(\mathrm{C})\end{array}$ \\
\hline A-1 & L1P787 & 62.86 & 91.31 & 82.21 \\
\hline A-2 & L2P481 & 61.05 & 85.74 & 74.38 \\
\hline A-3 & L2P498 & 59.92 & 82.74 & 70.75 \\
\hline A-4 & L1P789 & 59.34 & 76.57 & 69.43 \\
\hline A-5 & L1P7A0 & 66.37 & 99.95 & 89.40 \\
\hline A-6 & L2P482 & 64.69 & 95.86 & 80.78 \\
\hline A-7 & L2P499 & 63.13 & 91.40 & 75.99 \\
\hline A-8 & L1P7A1 & 61.61 & 84.44 & 74.33 \\
\hline \hline B-1 & L5P3B1 & 75.18 & 119.45 & 106.56 \\
\hline B-2 & L5P3B3 & 71.63 & 105.83 & 89.96 \\
\hline B-3 & L5P2C9 & 66.11 & 90.03 & 79.37 \\
\hline B-4 & L5P1A5 & 61.39 & 75.38 & 70.33 \\
\hline B-5 & L5P3B2 & 76.40 & 119.22 & 107.10 \\
\hline B-6 & L5P3C1 & 71.82 & 106.16 & 90.73 \\
\hline B-7 & L5P2C8 & 66.53 & 92.48 & 79.92 \\
\hline B-8 & L5P1B8 & 61.77 & 77.13 & 70.75 \\
\hline \hline D-1 & L5P3C2 & 78.40 & 118.14 & 105.41 \\
\hline D-2 & L5P3B4 & 74.19 & 104.84 & 90.04 \\
\hline D-3 & L5P2A3 & 68.42 & 89.98 & 79.61 \\
\hline D-4 & L5P1B7 & 63.04 & 74.95 & 70.75 \\
\hline D-5 & L5P3C3 & 78.22 & 110.80 & 101.45 \\
\hline D-6 & L5P3C6 & 74.10 & 100.04 & 87.85 \\
\hline D-7 & L5P2C0 & 68.10 & 86.69 & 78.11 \\
\hline D-8 & L5P1B9 & 63.13 & 73.92 & 69.96 \\
\hline & & & & \\
\hline
\end{tabular}


Table 25: As-run minimum, maximum and average plate surface temperatures over fuel zone on the north side of the plate for plates irradiated in Cycle 150B, BOC (0 EFPD)

\begin{tabular}{|c|c|c|c|c|}
\hline $\begin{array}{c}\text { Plate } \\
\text { Location }\end{array}$ & Plate ID & $\begin{array}{c}\text { Minimum } \\
\text { Temperature } \\
(\mathrm{C})\end{array}$ & $\begin{array}{c}\text { Maximum } \\
\text { Temperature } \\
(\mathrm{C})\end{array}$ & $\begin{array}{c}\text { Average } \\
\text { Temperature } \\
(\mathrm{C})\end{array}$ \\
\hline A-1 & L1P787 & 62.68 & 89.99 & 81.24 \\
\hline A-2 & L2P481 & 61.05 & 85.60 & 74.30 \\
\hline A-3 & L2P498 & 59.91 & 82.67 & 70.72 \\
\hline A-4 & L1P789 & 59.47 & 77.35 & 69.99 \\
\hline A-5 & L1P7A0 & 66.12 & 98.61 & 88.19 \\
\hline A-6 & L2P482 & 64.63 & 95.55 & 80.51 \\
\hline A-7 & L2P499 & 63.10 & 91.24 & 75.87 \\
\hline A-8 & L1P7A1 & 61.75 & 85.21 & 74.98 \\
\hline \hline B-1 & L5P3B1 & 74.75 & 116.56 & 104.08 \\
\hline B-2 & L5P3B3 & 71.52 & 105.53 & 89.47 \\
\hline B-3 & L5P2C9 & 66.04 & 89.53 & 79.04 \\
\hline B-4 & L5P1A5 & 61.51 & 76.28 & 70.97 \\
\hline B-5 & L5P3B2 & 75.90 & 116.48 & 104.50 \\
\hline B-6 & L5P3C1 & 71.53 & 104.97 & 89.77 \\
\hline B-7 & L5P2C8 & 66.32 & 91.65 & 79.24 \\
\hline B-8 & L5P1B8 & 61.85 & 77.64 & 71.24 \\
\hline D-1 & L5P3C2 & 77.88 & 115.19 & 102.90 \\
\hline D-2 & L5P3B4 & 73.72 & 103.82 & 88.80 \\
\hline D-3 & L5P2A3 & 67.98 & 89.21 & 78.67 \\
\hline D-4 & L5P1B7 & 63.06 & 75.44 & 71.02 \\
\hline D-5 & L5P3C3 & 77.65 & 108.01 & 99.04 \\
\hline D-6 & L5P3C6 & 73.51 & 98.47 & 86.19 \\
\hline D-7 & L5P2C0 & 67.65 & 85.29 & 76.86 \\
\hline D-8 & L5P1B9 & 63.08 & 73.98 & 70.05 \\
\hline & & & & \\
\hline
\end{tabular}


Table 26: As-run minimum, maximum and average plate surface temperatures over fuel zone on the south side of the plate for plates irradiated in Cycle 150B, MOC1 (18.0 EFPD)

\begin{tabular}{|c|c|c|c|c|}
\hline $\begin{array}{c}\text { Plate } \\
\text { Location }\end{array}$ & Plate ID & $\begin{array}{c}\text { Minimum } \\
\text { Temperature } \\
(\mathrm{C})\end{array}$ & $\begin{array}{c}\text { Maximum } \\
\text { Temperature } \\
(\mathrm{C})\end{array}$ & $\begin{array}{c}\text { Average } \\
\text { Temperature } \\
(\mathrm{C})\end{array}$ \\
\hline A-1 & L1P787 & 62.14 & 88.92 & 80.33 \\
\hline A-2 & L2P481 & 60.59 & 84.10 & 73.29 \\
\hline A-3 & L2P498 & 59.76 & 82.17 & 70.39 \\
\hline A-4 & L1P789 & 59.42 & 76.80 & 69.61 \\
\hline A-5 & L1P7A0 & 65.24 & 96.31 & 86.58 \\
\hline A-6 & L2P482 & 63.68 & 92.42 & 78.52 \\
\hline A-7 & L2P499 & 62.59 & 89.47 & 74.82 \\
\hline A-8 & L1P7A1 & 61.39 & 83.66 & 73.79 \\
\hline \hline B-1 & L5P3B1 & 71.96 & 109.44 & 98.60 \\
\hline B-2 & L5P3B3 & 68.80 & 97.53 & 84.31 \\
\hline B-3 & L5P2C9 & 64.35 & 84.80 & 75.70 \\
\hline B-4 & L5P1A5 & 60.63 & 73.10 & 68.59 \\
\hline B-5 & L5P3B2 & 72.99 & 109.26 & 99.04 \\
\hline B-6 & L5P3C1 & 69.05 & 97.97 & 85.14 \\
\hline B-7 & L5P2C8 & 64.80 & 87.25 & 76.39 \\
\hline B-8 & L5P1B8 & 61.03 & 74.92 & 69.15 \\
\hline \hline D-1 & L5P3C2 & 75.13 & 109.81 & 98.73 \\
\hline D-2 & L5P3B4 & 71.16 & 97.18 & 84.74 \\
\hline D-3 & L5P2A3 & 66.52 & 85.42 & 76.33 \\
\hline D-4 & L5P1B7 & 62.16 & 72.96 & 69.14 \\
\hline D-5 & L5P3C3 & 75.23 & 104.35 & 96.03 \\
\hline D-6 & L5P3C6 & 71.48 & 94.42 & 83.70 \\
\hline D-7 & L5P2C0 & 66.42 & 83.08 & 75.38 \\
\hline D-8 & L5P1B9 & 62.31 & 72.33 & 68.64 \\
\hline
\end{tabular}


Table 27: As-run minimum, maximum and average plate surface temperatures over fuel zone on the north side of the plate for plates irradiated in Cycle 150B, MOC1 (18.0 EFPD)

\begin{tabular}{|c|c|c|c|c|}
\hline $\begin{array}{c}\text { Plate } \\
\text { Location }\end{array}$ & Plate ID & $\begin{array}{c}\text { Minimum } \\
\text { Temperature } \\
(\mathrm{C})\end{array}$ & $\begin{array}{c}\text { Maximum } \\
\text { Temperature } \\
(\mathrm{C})\end{array}$ & $\begin{array}{c}\text { Average } \\
\text { Temperature } \\
(\mathrm{C})\end{array}$ \\
\hline A-1 & L1P787 & 61.98 & 87.68 & 79.43 \\
\hline A-2 & L2P481 & 60.59 & 83.99 & 73.22 \\
\hline A-3 & L2P498 & 59.76 & 82.11 & 70.37 \\
\hline A-4 & L1P789 & 59.55 & 77.59 & 70.17 \\
\hline A-5 & L1P7A0 & 65.01 & 95.10 & 85.47 \\
\hline A-6 & L2P482 & 63.63 & 92.15 & 78.29 \\
\hline A-7 & L2P499 & 62.57 & 89.35 & 74.73 \\
\hline A-8 & L1P7A1 & 61.53 & 84.41 & 74.44 \\
\hline \hline B-1 & L5P3B1 & 71.59 & 106.97 & 96.48 \\
\hline B-2 & L5P3B3 & 68.71 & 97.29 & 83.90 \\
\hline B-3 & L5P2C9 & 64.30 & 84.41 & 75.45 \\
\hline B-4 & L5P1A5 & 60.75 & 73.93 & 69.20 \\
\hline B-5 & L5P3B2 & 72.55 & 106.89 & 96.80 \\
\hline B-6 & L5P3C1 & 68.81 & 97.01 & 84.34 \\
\hline B-7 & L5P2C8 & 64.65 & 86.60 & 75.86 \\
\hline B-8 & L5P1B8 & 61.12 & 75.46 & 69.66 \\
\hline \hline D-1 & L5P3C2 & 74.68 & 107.24 & 96.52 \\
\hline D-2 & L5P3B4 & 70.78 & 96.36 & 83.73 \\
\hline D-3 & L5P2A3 & 66.18 & 84.81 & 75.59 \\
\hline D-4 & L5P1B7 & 62.20 & 73.50 & 69.46 \\
\hline D-5 & L5P3C3 & 74.72 & 101.86 & 93.86 \\
\hline D-6 & L5P3C6 & 71.01 & 93.15 & 82.33 \\
\hline D-7 & L5P2C0 & 66.07 & 81.97 & 74.39 \\
\hline D-8 & L5P1B9 & 62.30 & 72.50 & 68.83 \\
\hline & & & & \\
\hline
\end{tabular}


Table 28: As-run minimum, maximum and average plate surface temperatures over fuel zone on the south side of the plate for plates irradiated in Cycle 150B, MOC2 (31.0 EFPD)

\begin{tabular}{|c|c|c|c|c|}
\hline $\begin{array}{c}\text { Plate } \\
\text { Location }\end{array}$ & Plate ID & $\begin{array}{c}\text { Minimum } \\
\text { Temperature } \\
(\mathrm{C})\end{array}$ & $\begin{array}{c}\text { Maximum } \\
\text { Temperature } \\
(\mathrm{C})\end{array}$ & $\begin{array}{c}\text { Average } \\
\text { Temperature } \\
(\mathrm{C})\end{array}$ \\
\hline A-1 & L1P787 & 63.67 & 93.99 & 84.32 \\
\hline A-2 & L2P481 & 62.18 & 89.71 & 77.02 \\
\hline A-3 & L2P498 & 61.21 & 87.48 & 73.67 \\
\hline A-4 & L1P789 & 61.05 & 81.88 & 73.30 \\
\hline A-5 & L1P7A0 & 67.09 & 102.17 & 91.08 \\
\hline A-6 & L2P482 & 65.75 & 99.18 & 82.99 \\
\hline A-7 & L2P499 & 64.60 & 96.22 & 78.98 \\
\hline A-8 & L1P7A1 & 63.54 & 90.57 & 78.59 \\
\hline \hline B-1 & L5P3B1 & 74.57 & 116.82 & 104.54 \\
\hline B-2 & L5P3B3 & 71.76 & 105.52 & 89.83 \\
\hline B-3 & L5P2C9 & 66.84 & 91.39 & 80.43 \\
\hline B-4 & L5P1A5 & 62.50 & 77.89 & 72.30 \\
\hline B-5 & L5P3B2 & 75.97 & 117.33 & 105.62 \\
\hline B-6 & L5P3C1 & 72.10 & 106.38 & 90.95 \\
\hline B-7 & L5P2C8 & 67.53 & 94.83 & 81.59 \\
\hline B-8 & L5P1B8 & 63.07 & 80.43 & 73.23 \\
\hline \hline D-1 & L5P3C2 & 78.32 & 117.58 & 105.01 \\
\hline D-2 & L5P3B4 & 74.66 & 105.70 & 90.67 \\
\hline D-3 & L5P2A3 & 69.59 & 92.64 & 81.50 \\
\hline D-4 & L5P1B7 & 64.48 & 78.16 & 73.28 \\
\hline D-5 & L5P3C3 & 78.60 & 111.84 & 102.29 \\
\hline D-6 & L5P3C6 & 75.11 & 102.63 & 89.62 \\
\hline D-7 & L5P2C0 & 69.51 & 89.96 & 80.47 \\
\hline D-8 & L5P1B9 & 64.76 & 77.71 & 72.95 \\
\hline & & & & \\
\hline
\end{tabular}


Table 29: As-run minimum, maximum and average plate surface temperatures over fuel zone on the north side of the plate for plates irradiated in Cycle 150B, MOC2 (31.0 EFPD)

\begin{tabular}{|c|c|c|c|c|}
\hline $\begin{array}{c}\text { Plate } \\
\text { Location }\end{array}$ & Plate ID & $\begin{array}{c}\text { Minimum } \\
\text { Temperature } \\
(\mathrm{C})\end{array}$ & $\begin{array}{c}\text { Maximum } \\
\text { Temperature } \\
(\mathrm{C})\end{array}$ & $\begin{array}{c}\text { Average } \\
\text { Temperature } \\
(\mathrm{C})\end{array}$ \\
\hline A-1 & L1P787 & 63.48 & 92.60 & 83.29 \\
\hline A-2 & L2P481 & 62.18 & 89.58 & 76.95 \\
\hline A-3 & L2P498 & 61.21 & 87.42 & 73.65 \\
\hline A-4 & L1P789 & 61.20 & 82.84 & 73.97 \\
\hline A-5 & L1P7A0 & 66.83 & 100.78 & 89.84 \\
\hline A-6 & L2P482 & 65.69 & 98.91 & 82.74 \\
\hline A-7 & L2P499 & 64.58 & 96.11 & 78.89 \\
\hline A-8 & L1P7A1 & 63.71 & 91.50 & 79.37 \\
\hline \hline B-1 & L5P3B1 & 74.17 & 114.07 & 102.20 \\
\hline B-2 & L5P3B3 & 71.66 & 105.26 & 89.40 \\
\hline B-3 & L5P2C9 & 66.79 & 90.96 & 80.15 \\
\hline B-4 & L5P1A5 & 62.64 & 78.88 & 73.02 \\
\hline B-5 & L5P3B2 & 75.50 & 114.73 & 103.15 \\
\hline B-6 & L5P3C1 & 71.85 & 105.34 & 90.10 \\
\hline B-7 & L5P2C8 & 67.35 & 94.11 & 81.00 \\
\hline B-8 & L5P1B8 & 63.17 & 81.07 & 73.82 \\
\hline \hline D-1 & L5P3C2 & 77.84 & 114.73 & 102.59 \\
\hline D-2 & L5P3B4 & 74.25 & 104.80 & 89.56 \\
\hline D-3 & L5P2A3 & 69.20 & 91.96 & 80.67 \\
\hline D-4 & L5P1B7 & 64.52 & 78.76 & 73.67 \\
\hline D-5 & L5P3C3 & 78.06 & 109.11 & 99.94 \\
\hline D-6 & L5P3C6 & 74.59 & 101.24 & 88.11 \\
\hline D-7 & L5P2C0 & 69.11 & 88.70 & 79.35 \\
\hline D-8 & L5P1B9 & 64.74 & 77.91 & 73.17 \\
\hline & & & & \\
\hline
\end{tabular}


Table 30: As-run minimum, maximum and average plate surface temperatures over fuel zone on the south side of the plate for plates irradiated in Cycle 150B, EOC (41.9 EFPD)

\begin{tabular}{|c|c|c|c|c|}
\hline $\begin{array}{c}\text { Plate } \\
\text { Location }\end{array}$ & Plate ID & $\begin{array}{c}\text { Minimum } \\
\text { Temperature } \\
\text { (C) }\end{array}$ & $\begin{array}{c}\text { Maximum } \\
\text { Temperature } \\
\text { (C) }\end{array}$ & $\begin{array}{c}\text { Average } \\
\text { Temperature } \\
\text { (C) }\end{array}$ \\
\hline A-1 & L1P787 & 63.48 & 93.38 & 83.84 \\
\hline A-2 & L2P481 & 62.10 & 89.41 & 76.83 \\
\hline A-3 & L2P498 & 61.33 & 87.91 & 73.94 \\
\hline A-4 & L1P789 & 61.32 & 82.70 & 73.89 \\
\hline A-5 & L1P7A0 & 66.77 & 101.11 & 90.28 \\
\hline A-6 & L2P482 & 65.72 & 99.11 & 82.93 \\
\hline A-7 & L2P499 & 64.68 & 96.47 & 79.13 \\
\hline A-8 & L1P7A1 & 63.91 & 91.77 & 79.41 \\
\hline \hline B-1 & L5P3B1 & 74.09 & 115.33 & 103.35 \\
\hline B-2 & L5P3B3 & 71.61 & 105.08 & 89.52 \\
\hline B-3 & L5P2C9 & 66.80 & 91.21 & 80.31 \\
\hline B-4 & L5P1A5 & 62.67 & 78.29 & 72.61 \\
\hline B-5 & L5P3B2 & 75.44 & 115.78 & 104.37 \\
\hline B-6 & L5P3C1 & 71.79 & 105.42 & 90.30 \\
\hline B-7 & L5P2C8 & 67.54 & 94.79 & 81.56 \\
\hline B-8 & L5P1B8 & 63.25 & 80.89 & 73.56 \\
\hline D-1 & L5P3C2 & 77.84 & 116.34 & 104.02 \\
\hline D-2 & L5P3B4 & 74.40 & 105.07 & 90.20 \\
\hline D-3 & L5P2A3 & 69.59 & 92.66 & 81.51 \\
\hline D-4 & L5P1B7 & 64.67 & 78.63 & 73.65 \\
\hline D-5 & L5P3C3 & 78.24 & 111.12 & 101.68 \\
\hline D-6 & L5P3C6 & 74.90 & 102.22 & 89.29 \\
\hline D-7 & L5P2C0 & 69.53 & 90.02 & 80.51 \\
\hline D-8 & L5P1B9 & 64.94 & 78.13 & 73.28 \\
\hline & & & & \\
\hline
\end{tabular}


Table 31: As-run minimum, maximum and average plate surface temperatures over fuel zone on the north side of the plate for plates irradiated in Cycle 150B, EOC (41.9 EFPD)

\begin{tabular}{|c|c|c|c|c|}
\hline $\begin{array}{c}\text { Plate } \\
\text { Location }\end{array}$ & Plate ID & $\begin{array}{c}\text { Minimum } \\
\text { Temperature } \\
\text { (C) }\end{array}$ & $\begin{array}{c}\text { Maximum } \\
\text { Temperature } \\
(\mathrm{C})\end{array}$ & $\begin{array}{c}\text { Average } \\
\text { Temperature } \\
(\mathrm{C})\end{array}$ \\
\hline A-1 & L1P787 & 63.29 & 92.00 & 82.83 \\
\hline A-2 & L2P481 & 62.10 & 89.30 & 76.76 \\
\hline A-3 & L2P498 & 61.33 & 87.86 & 73.92 \\
\hline A-4 & L1P789 & 61.48 & 83.69 & 74.59 \\
\hline A-5 & L1P7A0 & 66.52 & 99.76 & 89.07 \\
\hline A-6 & L2P482 & 65.67 & 98.86 & 82.71 \\
\hline A-7 & L2P499 & 64.66 & 96.38 & 79.06 \\
\hline A-8 & L1P7A1 & 64.09 & 92.73 & 80.23 \\
\hline \hline B-1 & L5P3B1 & 73.69 & 112.64 & 101.07 \\
\hline B-2 & L5P3B3 & 71.52 & 104.84 & 89.12 \\
\hline B-3 & L5P2C9 & 66.76 & 90.81 & 80.06 \\
\hline B-4 & L5P1A5 & 62.81 & 79.31 & 73.36 \\
\hline B-5 & L5P3B2 & 74.98 & 113.26 & 101.98 \\
\hline B-6 & L5P3C1 & 71.55 & 104.44 & 89.50 \\
\hline B-7 & L5P2C8 & 67.37 & 94.11 & 81.01 \\
\hline B-8 & L5P1B8 & 63.35 & 81.56 & 74.19 \\
\hline \hline D-1 & L5P3C2 & 77.37 & 113.56 & 101.66 \\
\hline D-2 & L5P3B4 & 74.01 & 104.22 & 89.17 \\
\hline D-3 & L5P2A3 & 69.22 & 92.03 & 80.73 \\
\hline D-4 & L5P1B7 & 64.72 & 79.27 & 74.07 \\
\hline D-5 & L5P3C3 & 77.72 & 108.44 & 99.37 \\
\hline D-6 & L5P3C6 & 74.41 & 100.91 & 87.86 \\
\hline D-7 & L5P2C0 & 69.16 & 88.84 & 79.45 \\
\hline D-8 & L5P1B9 & 64.93 & 78.37 & 73.54 \\
\hline & & & & \\
\hline
\end{tabular}


Table 32: As-run minimum, maximum and average plate surface temperatures over fuel zone on the south side of the plate for plates irradiated in Cycle 151A, BOC (0.0 EFPD)

\begin{tabular}{|c|c|c|c|c|}
\hline $\begin{array}{c}\text { Plate } \\
\text { Location }\end{array}$ & Plate ID & $\begin{array}{c}\text { Minimum } \\
\text { Temperature }\end{array}$ & $\begin{array}{c}\text { Maximum } \\
\text { Temperature } \\
\text { (C) }\end{array}$ & $\begin{array}{c}\text { Average } \\
\text { Temperature } \\
(\mathrm{C})\end{array}$ \\
\hline A-1 & L1P787 & 61.07 & 85.35 & 77.54 \\
\hline A-2 & L2P481 & 59.67 & 80.87 & 71.13 \\
\hline A-3 & L2P498 & 58.73 & 78.42 & 68.08 \\
\hline A-4 & L1P789 & 58.33 & 73.40 & 67.15 \\
\hline A-5 & L1P7A0 & 63.68 & 91.40 & 82.74 \\
\hline A-6 & L2P482 & 62.30 & 87.89 & 75.54 \\
\hline A-7 & L2P499 & 61.28 & 85.13 & 72.14 \\
\hline A-8 & L1P7A1 & 60.12 & 79.64 & 71.00 \\
\hline B-1 & L5P3F8 & 71.87 & 112.38 & 100.78 \\
\hline B-2 & L5P3G2 & 68.07 & 100.28 & 86.12 \\
\hline B-3 & L5P2A4 & 63.77 & 86.96 & 76.37 \\
\hline B-4 & L5P1B5 & 59.93 & 72.99 & 68.23 \\
\hline B-5 & L5P3F0 & 74.30 & 119.67 & 105.48 \\
\hline B-6 & L5P3G3 & 71.05 & 106.29 & 89.91 \\
\hline B-7 & L5P2C7 & 65.40 & 91.85 & 79.07 \\
\hline B-8 & L5P1B0 & 60.67 & 75.70 & 70.05 \\
\hline \hline C-1 & L5P3B1 & 74.48 & 107.44 & 98.06 \\
\hline C-2 & L5P3B3 & 71.03 & 96.52 & 85.02 \\
\hline C-3 & L5P2C9 & 65.89 & 84.14 & 76.16 \\
\hline C-4 & L5P1A5 & 61.67 & 72.67 & 68.78 \\
\hline C-5 & L5P3B2 & 72.35 & 100.08 & 91.96 \\
\hline C-6 & L5P3C1 & 71.58 & 97.68 & 86.14 \\
\hline C-7 & L5P2C8 & 66.54 & 86.76 & 77.06 \\
\hline C-8 & L5P1B8 & 62.14 & 74.37 & 69.32 \\
\hline \hline D-1 & L5P3C2 & 77.19 & 107.18 & 97.64 \\
\hline D-2 & L5P3B4 & 73.61 & 97.47 & 86.07 \\
\hline D-3 & L5P2A3 & 68.29 & 85.34 & 77.21 \\
\hline D-4 & L5P1B7 & 63.45 & 73.21 & 69.81 \\
\hline D-5 & L5P3C3 & 77.61 & 103.50 & 96.12 \\
\hline D-6 & L5P3C6 & 73.77 & 94.44 & 84.81 \\
\hline D-7 & L5P2C0 & 68.22 & 83.40 & 76.45 \\
\hline D-8 & L5P1B9 & 63.61 & 72.62 & 69.32 \\
\hline
\end{tabular}


Table 33: As-run minimum, maximum and average plate surface temperatures over fuel zone on the north side of the plate for plates irradiated in Cycle 151A, BOC (0.0 EFPD)

\begin{tabular}{|c|c|c|c|c|}
\hline $\begin{array}{c}\text { Plate } \\
\text { Location }\end{array}$ & Plate ID & $\begin{array}{c}\text { Minimum } \\
\text { Temperature }\end{array}$ & $\begin{array}{c}\text { Maximum } \\
\text { Temperature } \\
\text { (C) }\end{array}$ & $\begin{array}{c}\text { Average } \\
\text { Temperature } \\
(\mathrm{C})\end{array}$ \\
\hline A-1 & L1P787 & 60.92 & 84.24 & 76.72 \\
\hline A-2 & L2P481 & 59.67 & 80.76 & 71.06 \\
\hline A-3 & L2P498 & 58.73 & 78.36 & 68.06 \\
\hline A-4 & L1P789 & 58.44 & 74.09 & 67.63 \\
\hline A-5 & L1P7A0 & 63.48 & 90.32 & 81.75 \\
\hline A-6 & L2P482 & 62.25 & 87.64 & 75.33 \\
\hline A-7 & L2P499 & 61.26 & 85.01 & 72.05 \\
\hline A-8 & L1P7A1 & 60.24 & 80.29 & 71.56 \\
\hline B-1 & L5P3F8 & 71.51 & 109.61 & 98.59 \\
\hline B-2 & L5P3G2 & 67.98 & 99.66 & 85.68 \\
\hline B-3 & L5P2A4 & 63.72 & 86.53 & 76.08 \\
\hline B-4 & L5P1B5 & 60.02 & 73.72 & 68.77 \\
\hline B-5 & L5P3F0 & 73.85 & 117.00 & 103.02 \\
\hline B-6 & L5P3G3 & 70.80 & 105.22 & 89.04 \\
\hline B-7 & L5P2C7 & 65.22 & 91.10 & 78.46 \\
\hline B-8 & L5P1B0 & 60.73 & 76.14 & 70.51 \\
\hline \hline C-1 & L5P3B1 & 74.05 & 104.98 & 95.90 \\
\hline C-2 & L5P3B3 & 70.69 & 95.62 & 83.93 \\
\hline C-3 & L5P2C9 & 65.62 & 83.12 & 75.29 \\
\hline C-4 & L5P1A5 & 61.67 & 73.08 & 69.01 \\
\hline C-5 & L5P3B2 & 69.61 & 94.37 & 83.46 \\
\hline C-6 & L5P3C1 & 71.07 & 96.03 & 84.69 \\
\hline C-7 & L5P2C8 & 66.14 & 85.51 & 75.94 \\
\hline C-8 & L5P1B8 & 62.09 & 74.50 & 69.45 \\
\hline \hline D-1 & L5P3C2 & 76.70 & 104.70 & 95.50 \\
\hline D-2 & L5P3B4 & 73.04 & 96.10 & 84.56 \\
\hline D-3 & L5P2A3 & 67.73 & 84.22 & 75.96 \\
\hline D-4 & L5P1B7 & 63.38 & 73.40 & 69.81 \\
\hline D-5 & L5P3C3 & 77.08 & 101.08 & 94.00 \\
\hline D-6 & L5P3C6 & 73.09 & 92.61 & 82.97 \\
\hline D-7 & L5P2C0 & 67.67 & 81.77 & 74.95 \\
\hline D-8 & L5P1B9 & 63.49 & 72.45 & 69.19 \\
\hline
\end{tabular}


Table 34: As-run minimum, maximum and average plate surface temperatures over fuel zone on the south side of the plate for plates irradiated in Cycle 151A, MOC1 (15.0 EFPD)

\begin{tabular}{|c|c|c|c|c|}
\hline $\begin{array}{c}\text { Plate } \\
\text { Location }\end{array}$ & Plate ID & $\begin{array}{c}\text { Minimum } \\
\text { Temperature } \\
(\mathrm{C})\end{array}$ & $\begin{array}{c}\text { Maximum } \\
\text { Temperature } \\
(\mathrm{C})\end{array}$ & $\begin{array}{c}\text { Average } \\
\text { Temperature } \\
(\mathrm{C})\end{array}$ \\
\hline A-1 & L1P787 & 60.51 & 83.46 & 76.06 \\
\hline A-2 & L2P481 & 59.50 & 80.25 & 70.72 \\
\hline A-3 & L2P498 & 58.58 & 77.85 & 67.73 \\
\hline A-4 & L1P789 & 58.13 & 72.76 & 66.69 \\
\hline A-5 & L1P7A0 & 62.89 & 88.82 & 80.72 \\
\hline A-6 & L2P482 & 61.87 & 86.41 & 74.57 \\
\hline A-7 & L2P499 & 61.00 & 84.14 & 71.54 \\
\hline A-8 & L1P7A1 & 59.76 & 78.43 & 70.16 \\
\hline \hline B-1 & L5P3F8 & 67.74 & 99.06 & 90.20 \\
\hline B-2 & L5P3G2 & 64.56 & 88.98 & 78.33 \\
\hline B-3 & L5P2A4 & 61.27 & 78.70 & 70.78 \\
\hline B-4 & L5P1B5 & 58.51 & 68.53 & 64.91 \\
\hline B-5 & L5P3F0 & 69.50 & 104.09 & 93.36 \\
\hline B-6 & L5P3G3 & 66.63 & 92.67 & 80.74 \\
\hline B-7 & L5P2C7 & 62.43 & 82.13 & 72.63 \\
\hline B-8 & L5P1B0 & 59.09 & 70.60 & 66.28 \\
\hline C-1 & L5P3B1 & 72.15 & 103.16 & 94.34 \\
\hline C-2 & L5P3B3 & 69.22 & 93.60 & 82.56 \\
\hline C-3 & L5P2C9 & 64.53 & 81.80 & 74.23 \\
\hline C-4 & L5P1A5 & 60.65 & 70.82 & 67.22 \\
\hline C-5 & L5P3B2 & 72.91 & 101.42 & 93.07 \\
\hline C-6 & L5P3C1 & 69.77 & 94.87 & 83.81 \\
\hline C-7 & L5P2C8 & 65.10 & 84.27 & 75.07 \\
\hline C-8 & L5P1B8 & 61.09 & 72.45 & 67.75 \\
\hline \hline D-1 & L5P3C2 & 74.87 & 103.50 & 94.41 \\
\hline D-2 & L5P3B4 & 71.59 & 94.27 & 83.47 \\
\hline D-3 & L5P2A3 & 66.76 & 83.04 & 75.27 \\
\hline D-4 & L5P1B7 & 62.34 & 71.53 & 68.32 \\
\hline D-5 & L5P3C3 & 75.25 & 99.95 & 92.92 \\
\hline D-6 & L5P3C6 & 71.99 & 92.26 & 82.81 \\
\hline D-7 & L5P2C0 & 66.71 & 81.21 & 74.57 \\
\hline D-8 & L5P1B9 & 62.48 & 70.98 & 67.87 \\
\hline
\end{tabular}


Table 35: As-run minimum, maximum and average plate surface temperatures over fuel zone on the north side of the plate for plates irradiated in Cycle 151A, MOC1 (15.0 EFPD)

\begin{tabular}{|c|c|c|c|c|}
\hline $\begin{array}{c}\text { Plate } \\
\text { Location }\end{array}$ & Plate ID & $\begin{array}{c}\text { Minimum } \\
\text { Temperature }\end{array}$ & $\begin{array}{c}\text { Maximum } \\
\text { Temperature } \\
(\mathrm{C})\end{array}$ & $\begin{array}{c}\text { Average } \\
\text { Temperature } \\
(\mathrm{C})\end{array}$ \\
\hline A-1 & L1P787 & 60.37 & 82.41 & 75.29 \\
\hline A-2 & L2P481 & 59.50 & 80.16 & 70.66 \\
\hline A-3 & L2P498 & 58.58 & 77.79 & 67.70 \\
\hline A-4 & L1P789 & 58.23 & 73.43 & 67.15 \\
\hline A-5 & L1P7A0 & 62.71 & 87.82 & 79.81 \\
\hline A-6 & L2P482 & 61.82 & 86.21 & 74.39 \\
\hline A-7 & L2P499 & 60.97 & 84.02 & 71.44 \\
\hline A-8 & L1P7A1 & 59.87 & 79.05 & 70.70 \\
\hline B-1 & L5P3F8 & 67.46 & 96.84 & 88.52 \\
\hline B-2 & L5P3G2 & 64.49 & 88.48 & 77.96 \\
\hline B-3 & L5P2A4 & 61.22 & 78.35 & 70.53 \\
\hline B-4 & L5P1B5 & 58.58 & 69.09 & 65.33 \\
\hline B-5 & L5P3F0 & 69.15 & 101.99 & 91.45 \\
\hline B-6 & L5P3G3 & 66.43 & 91.86 & 80.05 \\
\hline B-7 & L5P2C7 & 62.29 & 81.55 & 72.16 \\
\hline B-8 & L5P1B0 & 59.14 & 70.99 & 66.66 \\
\hline C-1 & L5P3B1 & 71.76 & 100.89 & 92.35 \\
\hline C-2 & L5P3B3 & 68.95 & 92.91 & 81.69 \\
\hline C-3 & L5P2C9 & 64.33 & 80.99 & 73.56 \\
\hline C-4 & L5P1A5 & 60.68 & 71.28 & 67.50 \\
\hline C-5 & L5P3B2 & 69.93 & 95.06 & 83.98 \\
\hline C-6 & L5P3C1 & 69.36 & 93.53 & 82.62 \\
\hline C-7 & L5P2C8 & 64.79 & 83.24 & 74.15 \\
\hline C-8 & L5P1B8 & 61.08 & 72.66 & 67.95 \\
\hline \hline D-1 & L5P3C2 & 74.43 & 101.19 & 92.41 \\
\hline D-2 & L5P3B4 & 71.09 & 93.15 & 82.20 \\
\hline D-3 & L5P2A3 & 66.29 & 82.10 & 74.22 \\
\hline D-4 & L5P1B7 & 62.30 & 71.81 & 68.40 \\
\hline D-5 & L5P3C3 & 74.77 & 97.69 & 90.95 \\
\hline D-6 & L5P3C6 & 71.41 & 90.74 & 81.24 \\
\hline D-7 & L5P2C0 & 66.24 & 79.80 & 73.28 \\
\hline D-8 & L5P1B9 & 62.40 & 70.90 & 67.82 \\
\hline
\end{tabular}


Table 36: As-run minimum, maximum and average plate surface temperatures over fuel zone on the south side of the plate for plates irradiated in Cycle 151A, MOC2 (34.0 EFPD)

\begin{tabular}{|c|c|c|c|c|}
\hline $\begin{array}{c}\text { Plate } \\
\text { Location }\end{array}$ & Plate ID & $\begin{array}{c}\text { Minimum } \\
\text { Temperature } \\
(\mathrm{C})\end{array}$ & $\begin{array}{c}\text { Maximum } \\
\text { Temperature } \\
(\mathrm{C})\end{array}$ & $\begin{array}{c}\text { Average } \\
\text { Temperature } \\
(\mathrm{C})\end{array}$ \\
\hline A-1 & L1P787 & 60.61 & 83.77 & 76.30 \\
\hline A-2 & L2P481 & 59.53 & 80.36 & 70.79 \\
\hline A-3 & L2P498 & 58.76 & 78.51 & 68.14 \\
\hline A-4 & L1P789 & 58.23 & 73.08 & 66.91 \\
\hline A-5 & L1P7A0 & 62.85 & 88.66 & 80.60 \\
\hline A-6 & L2P482 & 62.08 & 87.13 & 75.04 \\
\hline A-7 & L2P499 & 61.10 & 84.49 & 71.75 \\
\hline A-8 & L1P7A1 & 59.72 & 78.26 & 70.06 \\
\hline \hline B-1 & L5P3F8 & 68.12 & 100.30 & 91.16 \\
\hline B-2 & L5P3G2 & 65.00 & 90.37 & 79.30 \\
\hline B-3 & L5P2A4 & 61.68 & 80.02 & 71.68 \\
\hline B-4 & L5P1B5 & 58.69 & 69.09 & 65.33 \\
\hline B-5 & L5P3F0 & 69.82 & 105.10 & 94.14 \\
\hline B-6 & L5P3G3 & 67.22 & 94.43 & 81.95 \\
\hline B-7 & L5P2C7 & 62.81 & 83.33 & 73.44 \\
\hline B-8 & L5P1B0 & 59.29 & 71.19 & 66.72 \\
\hline C-1 & L5P3B1 & 72.00 & 102.31 & 93.70 \\
\hline C-2 & L5P3B3 & 69.42 & 93.91 & 82.81 \\
\hline C-3 & L5P2C9 & 64.53 & 81.55 & 74.10 \\
\hline C-4 & L5P1A5 & 60.65 & 70.61 & 67.09 \\
\hline C-5 & L5P3B2 & 71.58 & 97.68 & 86.14 \\
\hline C-6 & L5P3C1 & 69.93 & 95.06 & 83.98 \\
\hline C-7 & L5P2C8 & 65.22 & 84.38 & 75.19 \\
\hline C-8 & L5P1B8 & 61.16 & 72.50 & 67.81 \\
\hline \hline D-1 & L5P3C2 & 74.91 & 103.40 & 94.35 \\
\hline D-2 & L5P3B4 & 71.74 & 94.48 & 83.64 \\
\hline D-3 & L5P2A3 & 66.81 & 82.93 & 75.25 \\
\hline D-4 & L5P1B7 & 62.32 & 71.28 & 68.17 \\
\hline D-5 & L5P3C3 & 75.42 & 100.28 & 93.20 \\
\hline D-6 & L5P3C6 & 72.06 & 92.21 & 82.82 \\
\hline D-7 & L5P2C0 & 66.89 & 81.55 & 74.83 \\
\hline D-8 & L5P1B9 & 62.55 & 71.03 & 67.92 \\
\hline
\end{tabular}


Table 37: As-run minimum, maximum and average plate surface temperatures over fuel zone on the north side of the plate for plates irradiated in Cycle 151A, MOC2 (34.0 EFPD)

\begin{tabular}{|c|c|c|c|c|}
\hline $\begin{array}{c}\text { Plate } \\
\text { Location }\end{array}$ & Plate ID & $\begin{array}{c}\text { Minimum } \\
\text { Temperature } \\
(\mathrm{C})\end{array}$ & $\begin{array}{c}\text { Maximum } \\
\text { Temperature } \\
(\mathrm{C})\end{array}$ & $\begin{array}{c}\text { Average } \\
\text { Temperature } \\
\text { (C) }\end{array}$ \\
\hline A-1 & L1P787 & 60.46 & 82.71 & 75.52 \\
\hline A-2 & L2P481 & 59.53 & 80.27 & 70.74 \\
\hline A-3 & L2P498 & 58.76 & 78.46 & 68.11 \\
\hline A-4 & L1P789 & 58.33 & 73.75 & 67.39 \\
\hline A-5 & L1P7A0 & 62.67 & 87.67 & 79.69 \\
\hline A-6 & L2P482 & 62.04 & 86.93 & 74.86 \\
\hline A-7 & L2P499 & 61.08 & 84.36 & 71.65 \\
\hline A-8 & L1P7A1 & 59.84 & 78.88 & 70.58 \\
\hline \hline B-1 & L5P3F8 & 67.83 & 98.02 & 89.45 \\
\hline B-2 & L5P3G2 & 64.93 & 89.88 & 78.93 \\
\hline B-3 & L5P2A4 & 61.63 & 79.65 & 71.42 \\
\hline B-4 & L5P1B5 & 58.77 & 69.66 & 65.76 \\
\hline B-5 & L5P3F0 & 69.47 & 102.98 & 92.21 \\
\hline B-6 & L5P3G3 & 67.02 & 93.64 & 81.26 \\
\hline B-7 & L5P2C7 & 62.67 & 82.73 & 72.94 \\
\hline B-8 & L5P1B0 & 59.33 & 71.57 & 67.10 \\
\hline C-1 & L5P3B1 & 71.62 & 100.09 & 91.77 \\
\hline C-2 & L5P3B3 & 69.15 & 93.21 & 81.94 \\
\hline C-3 & L5P2C9 & 64.32 & 80.71 & 73.40 \\
\hline C-4 & L5P1A5 & 60.67 & 71.03 & 67.35 \\
\hline C-5 & L5P3B2 & 71.07 & 96.03 & 84.69 \\
\hline C-6 & L5P3C1 & 69.52 & 93.73 & 82.80 \\
\hline C-7 & L5P2C8 & 64.89 & 83.31 & 74.24 \\
\hline C-8 & L5P1B8 & 61.14 & 72.68 & 67.99 \\
\hline \hline D-1 & L5P3C2 & 74.48 & 101.11 & 92.37 \\
\hline D-2 & L5P3B4 & 71.24 & 93.36 & 82.37 \\
\hline D-3 & L5P2A3 & 66.34 & 81.98 & 74.17 \\
\hline D-4 & L5P1B7 & 62.28 & 71.54 & 68.22 \\
\hline D-5 & L5P3C3 & 74.94 & 98.04 & 91.24 \\
\hline D-6 & L5P3C6 & 71.48 & 90.69 & 81.25 \\
\hline D-7 & L5P2C0 & 66.41 & 80.12 & 73.53 \\
\hline D-8 & L5P1B9 & 62.46 & 70.93 & 67.86 \\
\hline
\end{tabular}


Table 38: As-run minimum, maximum and average plate surface temperatures over fuel zone on the south side of the plate for plates irradiated in Cycle 151A, EOC (56.1 EFPD)

\begin{tabular}{|c|c|c|c|c|}
\hline $\begin{array}{c}\text { Plate } \\
\text { Location }\end{array}$ & Plate ID & $\begin{array}{c}\text { Minimum } \\
\text { Temperature }\end{array}$ & $\begin{array}{c}\text { Maximum } \\
\text { Temperature } \\
(\mathrm{C})\end{array}$ & $\begin{array}{c}\text { Average } \\
\text { Temperature } \\
(\mathrm{C})\end{array}$ \\
\hline A-1 & L1P787 & 60.37 & 82.96 & 75.67 \\
\hline A-2 & L2P481 & 59.44 & 80.03 & 70.58 \\
\hline A-3 & L2P498 & 58.70 & 78.30 & 68.01 \\
\hline A-4 & L1P789 & 58.14 & 72.81 & 66.72 \\
\hline A-5 & L1P7A0 & 62.51 & 87.54 & 79.73 \\
\hline A-6 & L2P482 & 61.94 & 86.69 & 74.74 \\
\hline A-7 & L2P499 & 61.00 & 84.13 & 71.53 \\
\hline A-8 & L1P7A1 & 59.61 & 77.90 & 69.81 \\
\hline B-1 & L5P3F8 & 67.63 & 98.84 & 90.01 \\
\hline B-2 & L5P3G2 & 64.76 & 89.66 & 78.79 \\
\hline B-3 & L5P2A4 & 61.50 & 79.50 & 71.32 \\
\hline B-4 & L5P1B5 & 58.56 & 68.71 & 65.04 \\
\hline B-5 & L5P3F0 & 69.28 & 103.43 & 92.85 \\
\hline B-6 & L5P3G3 & 66.92 & 93.61 & 81.37 \\
\hline B-7 & L5P2C7 & 62.61 & 82.72 & 73.03 \\
\hline B-8 & L5P1B0 & 59.13 & 70.74 & 66.38 \\
\hline \hline C-1 & L5P3B1 & 71.45 & 100.91 & 92.56 \\
\hline C-2 & L5P3B3 & 69.13 & 93.31 & 82.35 \\
\hline C-3 & L5P2C9 & 64.34 & 81.12 & 73.77 \\
\hline C-4 & L5P1A5 & 60.48 & 70.23 & 66.79 \\
\hline C-5 & L5P3B2 & 72.92 & 101.71 & 93.28 \\
\hline C-6 & L5P3C1 & 69.61 & 94.37 & 83.46 \\
\hline C-7 & L5P2C8 & 65.02 & 83.92 & 74.84 \\
\hline C-8 & L5P1B8 & 60.99 & 72.08 & 67.49 \\
\hline \hline D-1 & L5P3C2 & 74.32 & 102.07 & 93.26 \\
\hline D-2 & L5P3B4 & 71.41 & 93.86 & 83.16 \\
\hline D-3 & L5P2A3 & 66.57 & 82.48 & 74.90 \\
\hline D-4 & L5P1B7 & 62.16 & 70.99 & 67.91 \\
\hline D-5 & L5P3C3 & 74.85 & 99.13 & 92.22 \\
\hline D-6 & L5P3C6 & 71.70 & 91.61 & 82.34 \\
\hline D-7 & L5P2C0 & 66.66 & 81.12 & 74.49 \\
\hline D-8 & L5P1B9 & 62.38 & 70.73 & 67.67 \\
\hline
\end{tabular}


Table 39: As-run minimum, maximum and average plate surface temperatures over fuel zone on the north side of the plate for plates irradiated in Cycle 151A, EOC (56.1 EFPD)

\begin{tabular}{|c|c|c|c|c|}
\hline $\begin{array}{c}\text { Plate } \\
\text { Location }\end{array}$ & Plate ID & $\begin{array}{c}\text { Minimum } \\
\text { Temperature }\end{array}$ & $\begin{array}{c}\text { Maximum } \\
\text { Temperature } \\
(\mathrm{C})\end{array}$ & $\begin{array}{c}\text { Average } \\
\text { Temperature } \\
(\mathrm{C})\end{array}$ \\
\hline A-1 & L1P787 & 60.23 & 81.93 & 74.92 \\
\hline A-2 & L2P481 & 59.44 & 79.95 & 70.52 \\
\hline A-3 & L2P498 & 58.70 & 78.25 & 67.98 \\
\hline A-4 & L1P789 & 58.25 & 73.48 & 67.19 \\
\hline A-5 & L1P7A0 & 62.33 & 86.59 & 78.85 \\
\hline A-6 & L2P482 & 61.91 & 86.50 & 74.58 \\
\hline A-7 & L2P499 & 60.98 & 84.00 & 71.44 \\
\hline A-8 & L1P7A1 & 59.73 & 78.51 & 70.32 \\
\hline B-1 & L5P3F8 & 67.36 & 96.65 & 88.36 \\
\hline B-2 & L5P3G2 & 64.69 & 89.20 & 78.45 \\
\hline B-3 & L5P2A4 & 61.45 & 79.14 & 71.07 \\
\hline B-4 & L5P1B5 & 58.63 & 69.27 & 65.46 \\
\hline B-5 & L5P3F0 & 68.95 & 101.40 & 91.00 \\
\hline B-6 & L5P3G3 & 66.73 & 92.85 & 80.71 \\
\hline B-7 & L5P2C7 & 62.47 & 82.13 & 72.54 \\
\hline B-8 & L5P1B0 & 59.18 & 71.11 & 66.75 \\
\hline \hline C-1 & L5P3B1 & 71.09 & 98.77 & 90.70 \\
\hline C-2 & L5P3B3 & 68.88 & 92.65 & 81.52 \\
\hline C-3 & L5P2C9 & 64.13 & 80.30 & 73.09 \\
\hline C-4 & L5P1A5 & 60.50 & 70.65 & 67.04 \\
\hline C-5 & L5P3B2 & 69.77 & 94.87 & 83.81 \\
\hline C-6 & L5P3C1 & 69.22 & 93.11 & 82.33 \\
\hline C-7 & L5P2C8 & 64.69 & 82.87 & 73.91 \\
\hline C-8 & L5P1B8 & 60.97 & 72.27 & 67.67 \\
\hline \hline D-1 & L5P3C2 & 73.91 & 99.86 & 91.35 \\
\hline D-2 & L5P3B4 & 70.91 & 92.80 & 81.94 \\
\hline D-3 & L5P2A3 & 66.11 & 81.56 & 73.85 \\
\hline D-4 & L5P1B7 & 62.11 & 71.24 & 67.97 \\
\hline D-5 & L5P3C3 & 74.39 & 96.96 & 90.33 \\
\hline D-6 & L5P3C6 & 71.16 & 90.17 & 80.83 \\
\hline D-7 & L5P2C0 & 66.19 & 79.73 & 73.22 \\
\hline D-8 & L5P1B9 & 62.29 & 70.64 & 67.61 \\
\hline
\end{tabular}


Table 40: As-run minimum, maximum and average plate surface temperatures over fuel zone on the north side of the plate for plates irradiated in Cycle 151B, BOC (0.0 EFPD)

\begin{tabular}{|c|c|c|c|c|}
\hline $\begin{array}{c}\text { Plate } \\
\text { Location }\end{array}$ & Plate ID & $\begin{array}{c}\text { Minimum } \\
\text { Temperature } \\
\text { (C) }\end{array}$ & $\begin{array}{c}\text { Maximum } \\
\text { Temperature } \\
(\mathrm{C})\end{array}$ & $\begin{array}{c}\text { Average } \\
\text { Temperature } \\
\text { (C) }\end{array}$ \\
\hline \hline D-1 & L5P3C2 & 69.70 & 108.74 & 96.18 \\
\hline D-2 & L5P3B4 & 67.10 & 99.27 & 83.73 \\
\hline D-3 & L5P2A3 & 62.64 & 85.30 & 74.31 \\
\hline D-4 & L5P1B7 & 58.54 & 71.09 & 66.56 \\
\hline D-5 & L5P3C3 & 69.68 & 102.78 & 93.30 \\
\hline D-6 & L5P3C6 & 67.06 & 94.57 & 81.58 \\
\hline D-7 & L5P2C0 & 62.34 & 81.76 & 72.74 \\
\hline D-8 & L5P1B9 & 58.49 & 69.89 & 65.69 \\
\hline
\end{tabular}

Table 41: As-run minimum, maximum and average plate surface temperatures over fuel zone on the south side of the plate for plates irradiated in Cycle 151B, BOC (0.0 EFPD)

\begin{tabular}{|c|c|c|c|c|}
\hline $\begin{array}{c}\text { Plate } \\
\text { Location }\end{array}$ & Plate ID & $\begin{array}{c}\text { Minimum } \\
\text { Temperature } \\
(\mathrm{C})\end{array}$ & $\begin{array}{c}\text { Maximum } \\
\text { Temperature } \\
(\mathrm{C})\end{array}$ & $\begin{array}{c}\text { Average } \\
\text { Temperature } \\
(\mathrm{C})\end{array}$ \\
\hline \hline D-1 & L5P3C2 & 69.32 & 106.20 & 94.12 \\
\hline D-2 & L5P3B4 & 66.95 & 99.18 & 83.49 \\
\hline D-3 & L5P2A3 & 62.56 & 85.34 & 74.23 \\
\hline D-4 & L5P1B7 & 58.71 & 72.12 & 67.32 \\
\hline D-5 & L5P3C3 & 69.26 & 100.38 & 91.28 \\
\hline D-6 & L5P3C6 & 66.90 & 94.14 & 80.97 \\
\hline D-7 & L5P2C0 & 62.25 & 81.27 & 72.37 \\
\hline D-8 & L5P1B9 & 58.63 & 70.52 & 66.30 \\
\hline
\end{tabular}

Table 42: As-run minimum, maximum and average plate surface temperatures over fuel zone on the north side of the plate for plates irradiated in Cycle 151B, MOC1 (23.0 EFPD)

\begin{tabular}{|c|c|c|c|c|}
\hline $\begin{array}{c}\text { Plate } \\
\text { Location }\end{array}$ & Plate ID & $\begin{array}{c}\text { Minimum } \\
\text { Temperature } \\
\text { (C) }\end{array}$ & $\begin{array}{c}\text { Maximum } \\
\text { Temperature } \\
\text { (C) }\end{array}$ & $\begin{array}{c}\text { Average } \\
\text { Temperature } \\
\text { (C) }\end{array}$ \\
\hline \hline D-1 & L5P3C2 & 68.64 & 105.27 & 93.59 \\
\hline D-2 & L5P3B4 & 66.30 & 96.79 & 82.08 \\
\hline D-3 & L5P2A3 & 62.12 & 83.66 & 73.22 \\
\hline D-4 & L5P1B7 & 58.29 & 70.28 & 65.95 \\
\hline D-5 & L5P3C3 & 68.98 & 100.83 & 91.76 \\
\hline D-6 & L5P3C6 & 66.63 & 93.48 & 80.79 \\
\hline D-7 & L5P2C0 & 62.01 & 80.85 & 72.10 \\
\hline D-8 & L5P1B9 & 58.34 & 69.48 & 65.37 \\
\hline
\end{tabular}


Table 43: As-run minimum, maximum and average plate surface temperatures over fuel zone on the south side of the plate for plates irradiated in Cycle 151B, MOC1 (23.0 EFPD)

\begin{tabular}{|c|c|c|c|c|}
\hline $\begin{array}{c}\text { Plate } \\
\text { Location }\end{array}$ & Plate ID & $\begin{array}{c}\text { Minimum } \\
\text { Temperature } \\
\text { (C) }\end{array}$ & $\begin{array}{c}\text { Maximum } \\
\text { Temperature } \\
\text { (C) }\end{array}$ & $\begin{array}{c}\text { Average } \\
\text { Temperature } \\
\text { (C) }\end{array}$ \\
\hline \hline D-1 & L5P3C2 & 68.29 & 102.91 & 91.65 \\
\hline D-2 & L5P3B4 & 66.16 & 96.71 & 81.85 \\
\hline D-3 & L5P2A3 & 62.05 & 83.71 & 73.15 \\
\hline D-4 & L5P1B7 & 58.46 & 71.29 & 66.69 \\
\hline D-5 & L5P3C3 & 68.58 & 98.57 & 89.82 \\
\hline D-6 & L5P3C6 & 66.48 & 93.07 & 80.22 \\
\hline D-7 & L5P2C0 & 61.93 & 80.39 & 71.76 \\
\hline D-8 & L5P1B9 & 58.48 & 70.11 & 65.99 \\
\hline
\end{tabular}

Table 44: As-run minimum, maximum and average plate surface temperatures over fuel zone on the north side of the plate for plates irradiated in Cycle 151B, MOC2 (39.0 EFPD)

\begin{tabular}{|c|c|c|c|c|}
\hline $\begin{array}{c}\text { Plate } \\
\text { Location }\end{array}$ & Plate ID & $\begin{array}{c}\text { Minimum } \\
\text { Temperature } \\
\text { (C) }\end{array}$ & $\begin{array}{c}\text { Maximum } \\
\text { Temperature } \\
\text { (C) }\end{array}$ & $\begin{array}{c}\text { Average } \\
\text { Temperature } \\
\text { (C) }\end{array}$ \\
\hline \hline D-1 & L5P3C2 & 70.00 & 109.71 & 96.92 \\
\hline D-2 & L5P3B4 & 67.92 & 101.88 & 85.46 \\
\hline D-3 & L5P2A3 & 63.42 & 87.81 & 75.98 \\
\hline D-4 & L5P1B7 & 59.03 & 72.67 & 67.74 \\
\hline D-5 & L5P3C3 & 70.41 & 105.05 & 95.09 \\
\hline D-6 & L5P3C6 & 68.27 & 98.15 & 83.99 \\
\hline D-7 & L5P2C0 & 63.32 & 84.72 & 74.77 \\
\hline D-8 & L5P1B9 & 59.10 & 71.84 & 67.15 \\
\hline
\end{tabular}

Table 45: As-run minimum, maximum and average plate surface temperatures over fuel zone on the south side of the plate for plates irradiated in Cycle 151B, MOC2 (39.0 EFPD)

\begin{tabular}{|c|c|c|c|c|}
\hline $\begin{array}{c}\text { Plate } \\
\text { Location }\end{array}$ & Plate ID & $\begin{array}{c}\text { Minimum } \\
\text { Temperature } \\
\text { (C) }\end{array}$ & $\begin{array}{c}\text { Maximum } \\
\text { Temperature } \\
\text { (C) }\end{array}$ & $\begin{array}{c}\text { Average } \\
\text { Temperature } \\
\text { (C) }\end{array}$ \\
\hline \hline D-1 & L5P3C2 & 69.62 & 107.13 & 94.83 \\
\hline D-2 & L5P3B4 & 67.78 & 101.80 & 85.22 \\
\hline D-3 & L5P2A3 & 63.34 & 87.86 & 75.89 \\
\hline D-4 & L5P1B7 & 59.21 & 73.74 & 68.54 \\
\hline D-5 & L5P3C3 & 69.99 & 102.57 & 93.00 \\
\hline D-6 & L5P3C6 & 68.12 & 97.73 & 83.41 \\
\hline D-7 & L5P2C0 & 63.23 & 84.21 & 74.38 \\
\hline D-8 & L5P1B9 & 59.25 & 72.50 & 67.80 \\
\hline
\end{tabular}


Table 46: As-run minimum, maximum and average plate surface temperatures over fuel zone on the north side of the plate for plates irradiated in Cycle 151B, EOC (51.3 EFPD)

\begin{tabular}{|c|c|c|c|c|}
\hline $\begin{array}{c}\text { Plate } \\
\text { Location }\end{array}$ & Plate ID & $\begin{array}{c}\text { Minimum } \\
\text { Temperature } \\
(\mathrm{C})\end{array}$ & $\begin{array}{c}\text { Maximum } \\
\text { Temperature } \\
(\mathrm{C})\end{array}$ & $\begin{array}{c}\text { Average } \\
\text { Temperature } \\
(\mathrm{C})\end{array}$ \\
\hline \hline D-1 & L5P3C2 & 69.28 & 107.35 & 95.15 \\
\hline D-2 & L5P3B4 & 67.38 & 100.23 & 84.36 \\
\hline D-3 & L5P2A3 & 63.03 & 86.58 & 75.16 \\
\hline D-4 & L5P1B7 & 58.81 & 71.96 & 67.21 \\
\hline D-5 & L5P3C3 & 69.77 & 103.15 & 93.59 \\
\hline D-6 & L5P3C6 & 67.78 & 96.78 & 83.04 \\
\hline D-7 & L5P2C0 & 62.97 & 83.69 & 74.06 \\
\hline D-8 & L5P1B9 & 58.90 & 71.22 & 66.68 \\
\hline
\end{tabular}

Table 47: As-run minimum, maximum and average plate surface temperatures over fuel zone on the south side of the plate for plates irradiated in Cycle 151B, EOC (51.3 EFPD)

\begin{tabular}{|c|c|c|c|c|}
\hline $\begin{array}{c}\text { Plate } \\
\text { Location }\end{array}$ & Plate ID & $\begin{array}{c}\text { Minimum } \\
\text { Temperature } \\
(\mathrm{C})\end{array}$ & $\begin{array}{c}\text { Maximum } \\
\text { Temperature } \\
(\mathrm{C})\end{array}$ & $\begin{array}{c}\text { Average } \\
\text { Temperature } \\
(\mathrm{C})\end{array}$ \\
\hline \hline D-1 & L5P3C2 & 68.91 & 104.88 & 93.15 \\
\hline D-2 & L5P3B4 & 67.25 & 100.15 & 84.13 \\
\hline D-3 & L5P2A3 & 62.96 & 86.63 & 75.08 \\
\hline D-4 & L5P1B7 & 58.99 & 73.01 & 67.99 \\
\hline D-5 & L5P3C3 & 69.36 & 100.77 & 91.58 \\
\hline D-6 & L5P3C6 & 67.63 & 96.39 & 82.48 \\
\hline D-7 & L5P2C0 & 62.88 & 83.20 & 73.69 \\
\hline D-8 & L5P1B9 & 59.04 & 71.87 & 67.33 \\
\hline
\end{tabular}




\section{REFERENCES}

1. Wachs, D. M, "RERTR Fuel Development and Qualification Plan," INL/EXT-05-0101, Revision 4, August 2009.

2. RERTR Project Personnel, "RERTR-12 As-Built Data Package (Plates)” RERTR-12, January 2010.

3. RERTR Project Personnel, "RERTR-12 Y-Capsules As-Built Data Package,"RERTR-12, Job ID 3331415, September 2010.

4. D. M. Perez, G. S. Chang, "RERTR-12-3 Cycle 150B As-Run Physics Analysis Results and RERTR12-4 Cycle 151A Projected Physics Analysis Results for Use in Thermal and Oxide Growth Evaluations," ECAR-1754, December 2011.

5. D. M. Perez, G. S. Chang, "RERTR-12-4 Cycle 151A As-Run Physics Analysis Results and RERTR12-5 Cycle 151B Projected Physics Analysis Results for Use in Thermal and Oxide Growth Evaluations," ECAR-1811, February 2012.

6. D. M. Perez, G. S. Chang, "RERTR-12-5 Cycle 151B As-Run Physics Analysis for Use in Thermal and Oxide Growth Evaluations," ECAR-2016, August 2012.

7. D.M. Wachs, "RERTR-Large-B Position Irradiation Vehicle Flow Test" EDF-8292, July 2007.

8. D. M. Wachs, "Thermal Analysis of the RERTR-9B Irradiation Test,” EDF-8083, July 2007.

9. P. E. Murray, "Validation of ABAQUS Standard 6.7-3 Heat Transfer", ECAR-131, January 2008.

10. R. H. Perry, D. W. Green, "Perry's Chemical Engineer's Handbook," $7^{\text {th }}$ Edition, McGraw-Hill, 1997.

11. G. A. Roth, "As-Run Thermal Analysis for the RERTR-12 Experiment Irradiated in the ATR" ECAR-2019, August 2012. 
Appendix A

\section{Individual Plate Power and Fission Density Plots}




\section{Appendix A \\ Individual Plate Power and Fission Density Plots}

A-1. Capsule Z

\section{Z-1 (L1P787)}

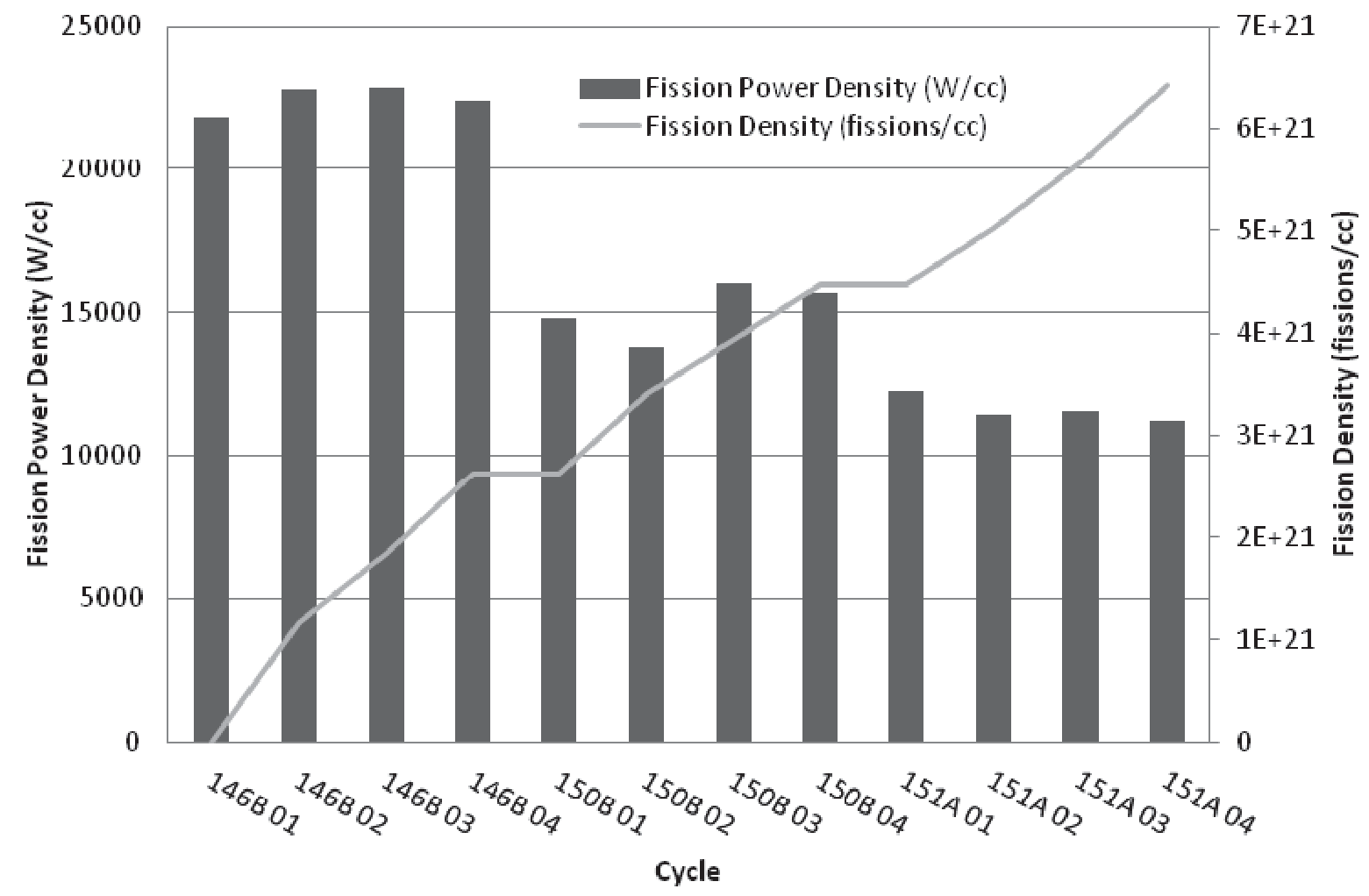



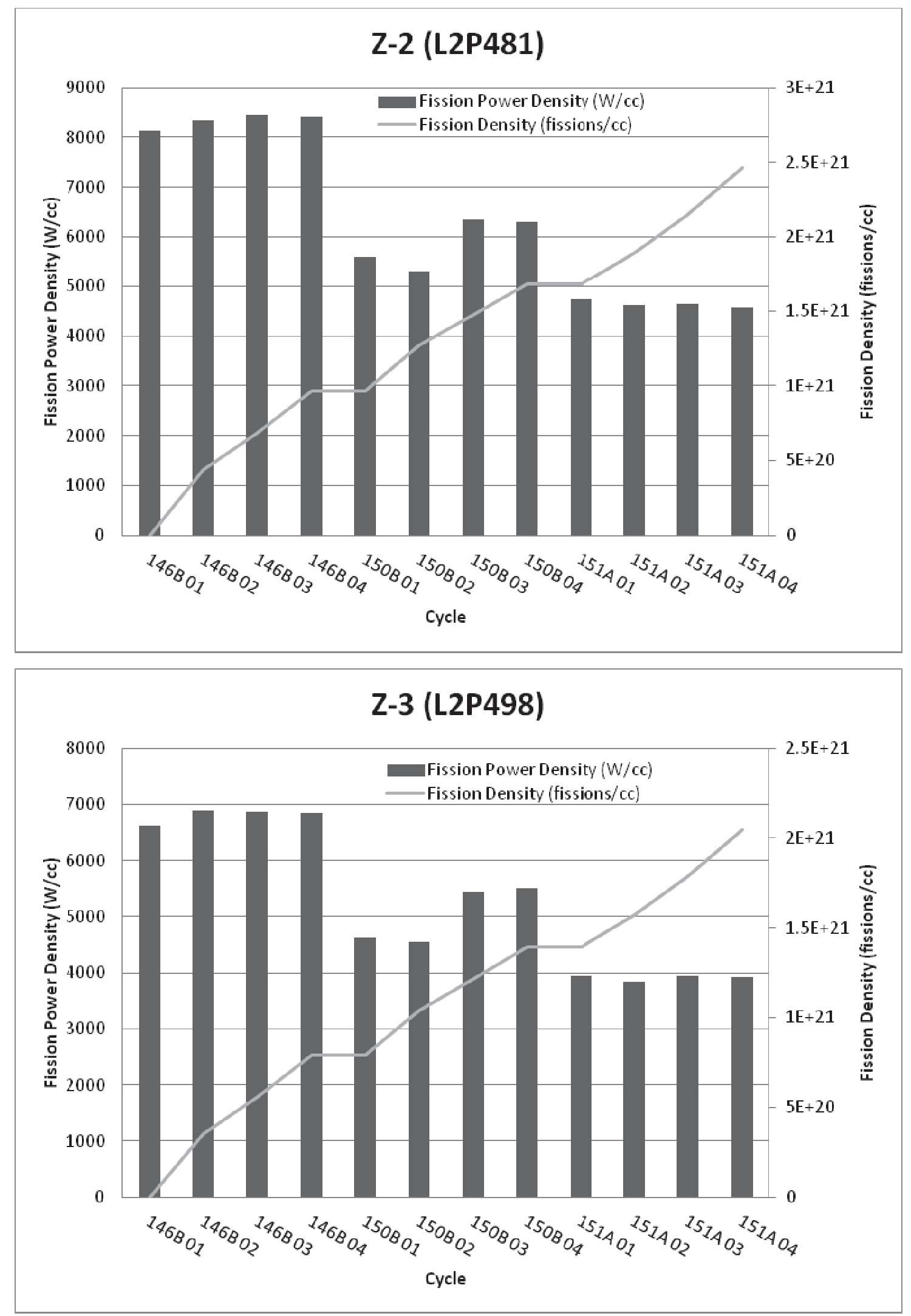

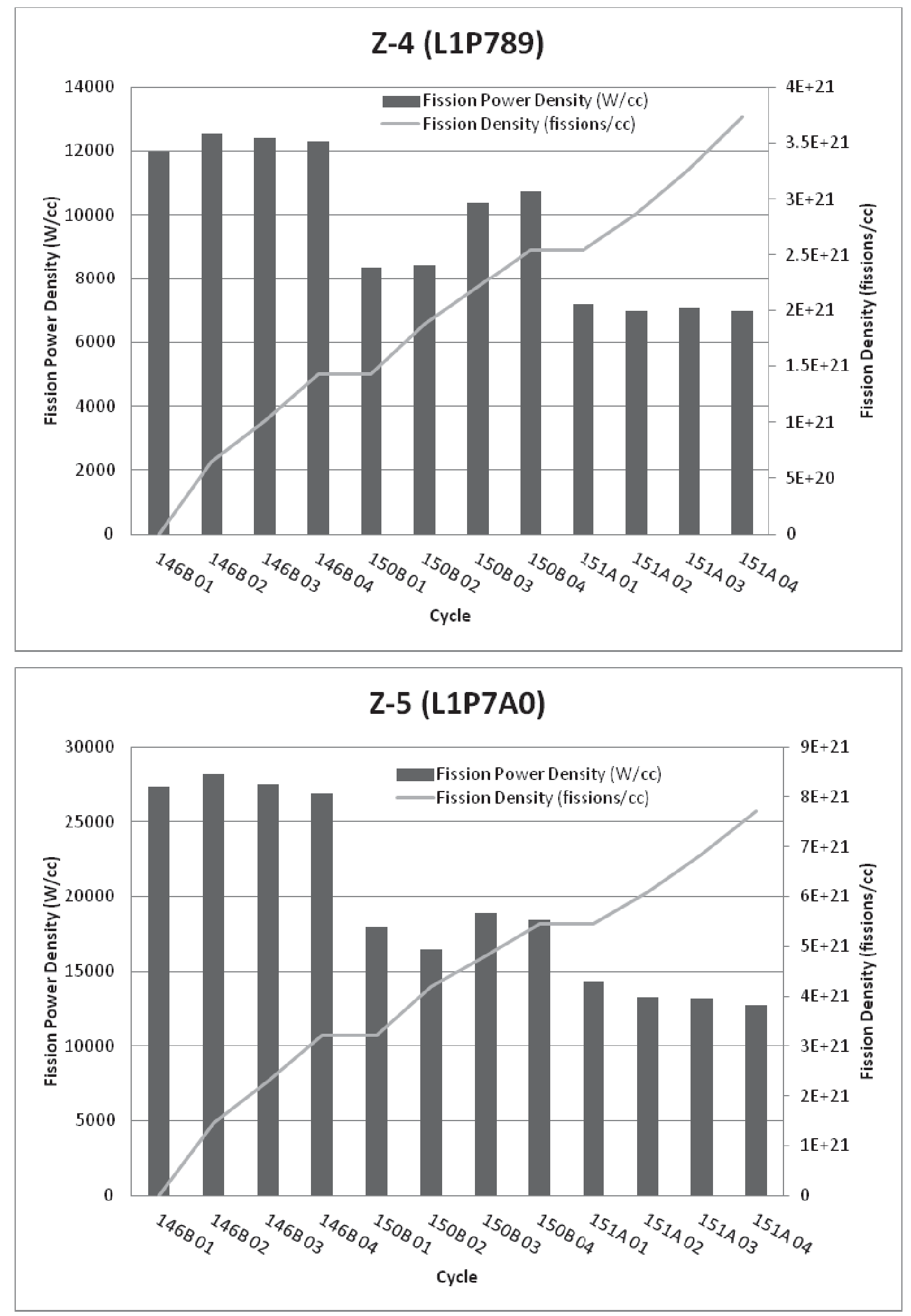

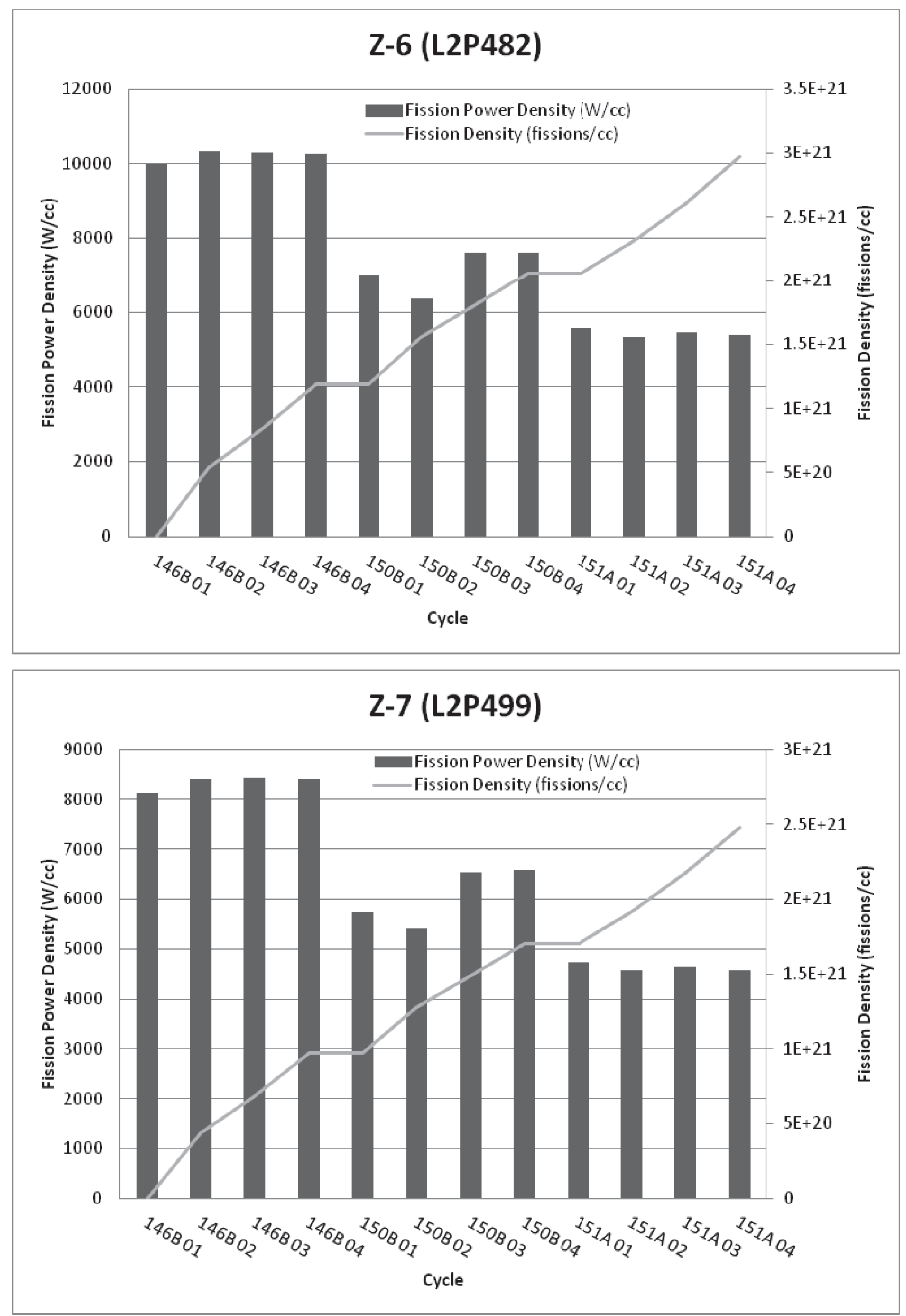


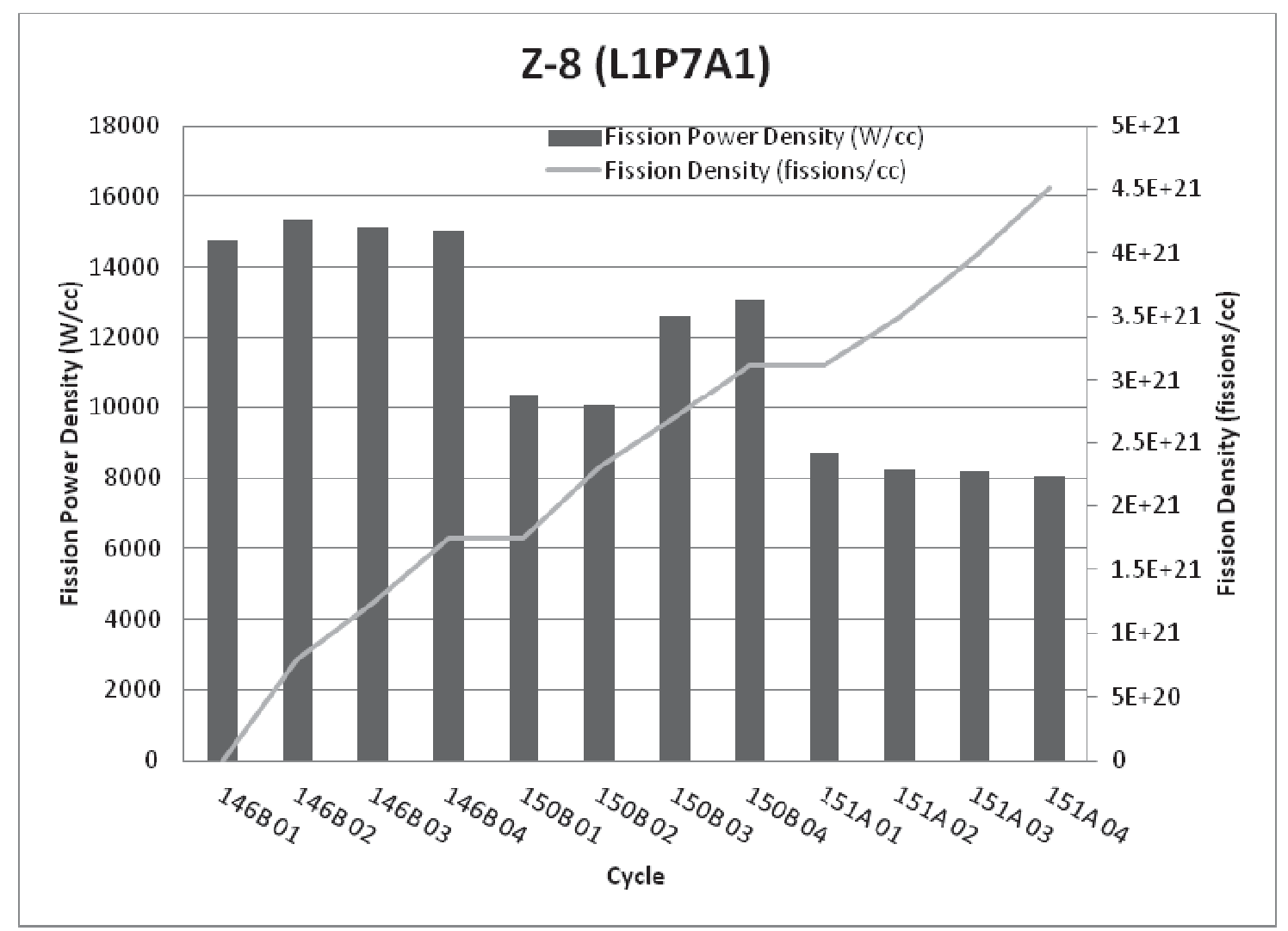




\section{A-2. Capsule Y-1}

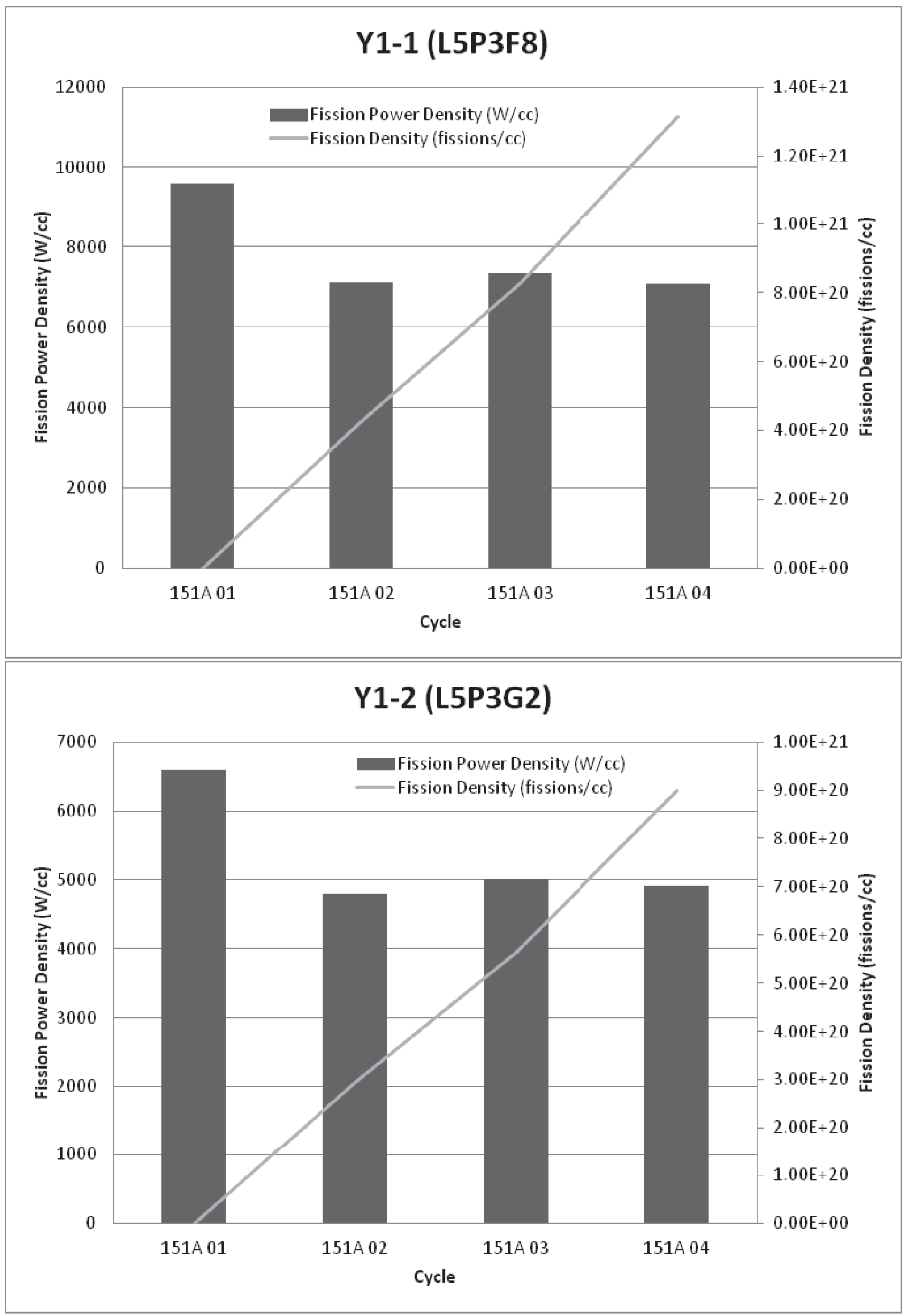



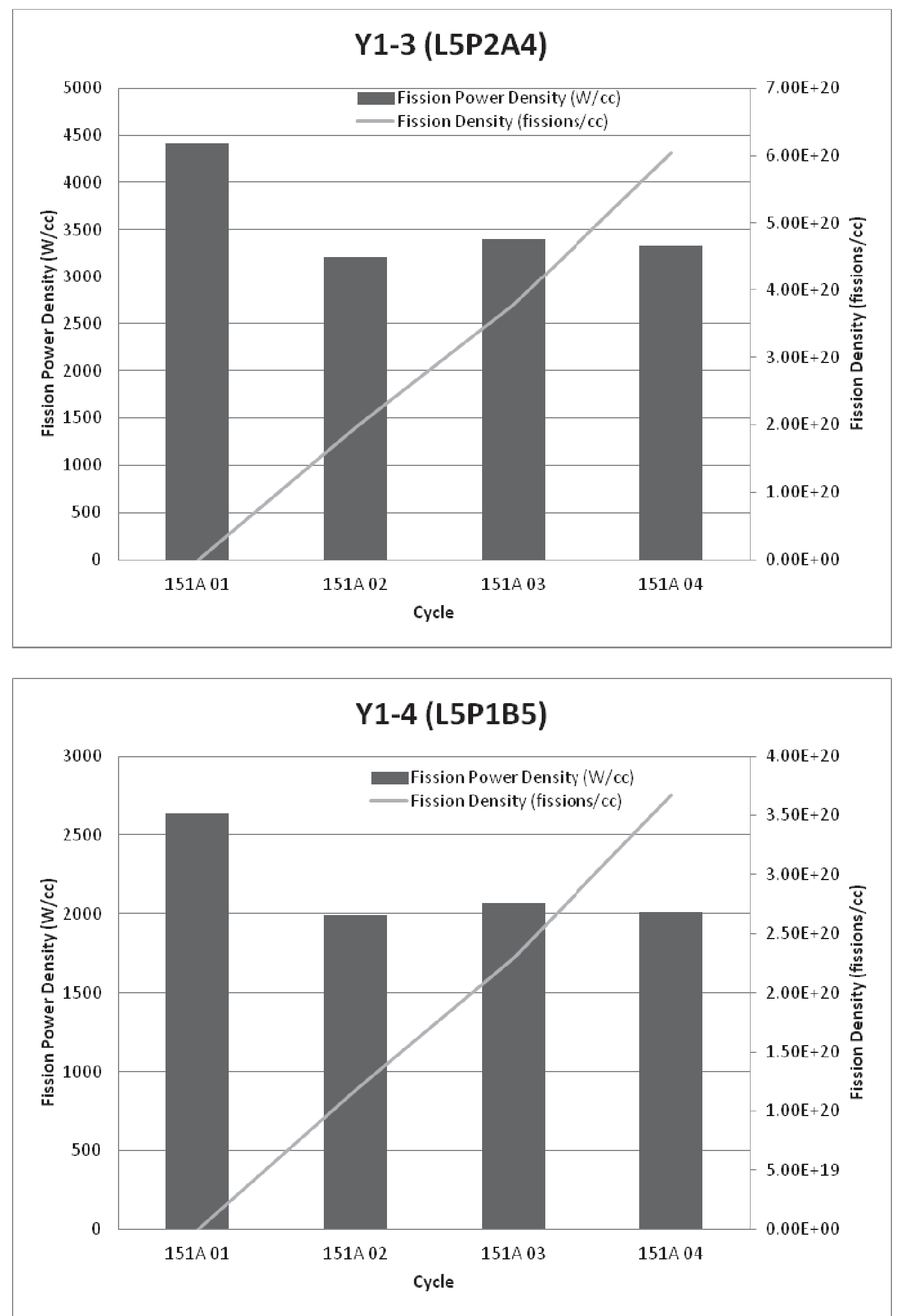

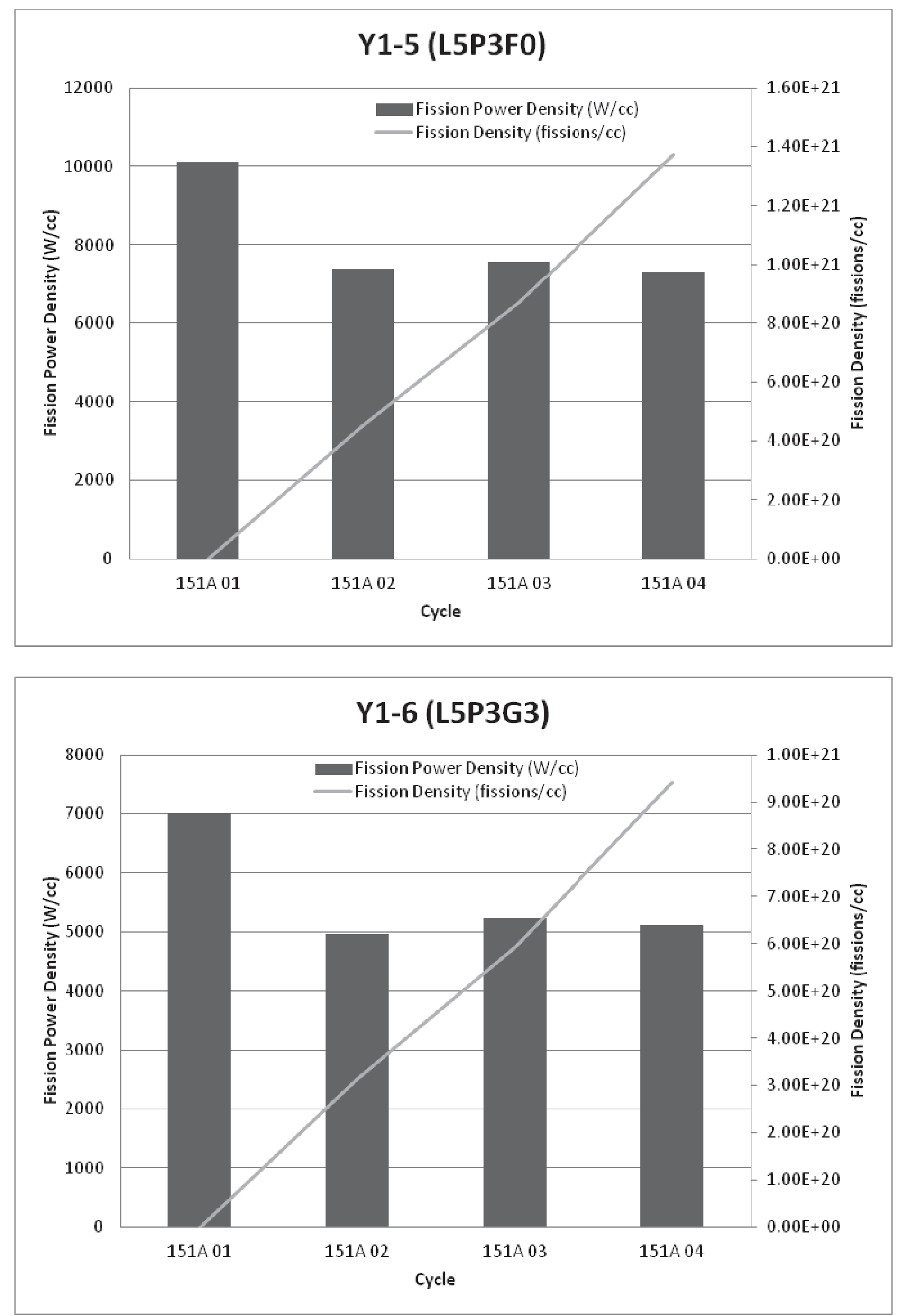

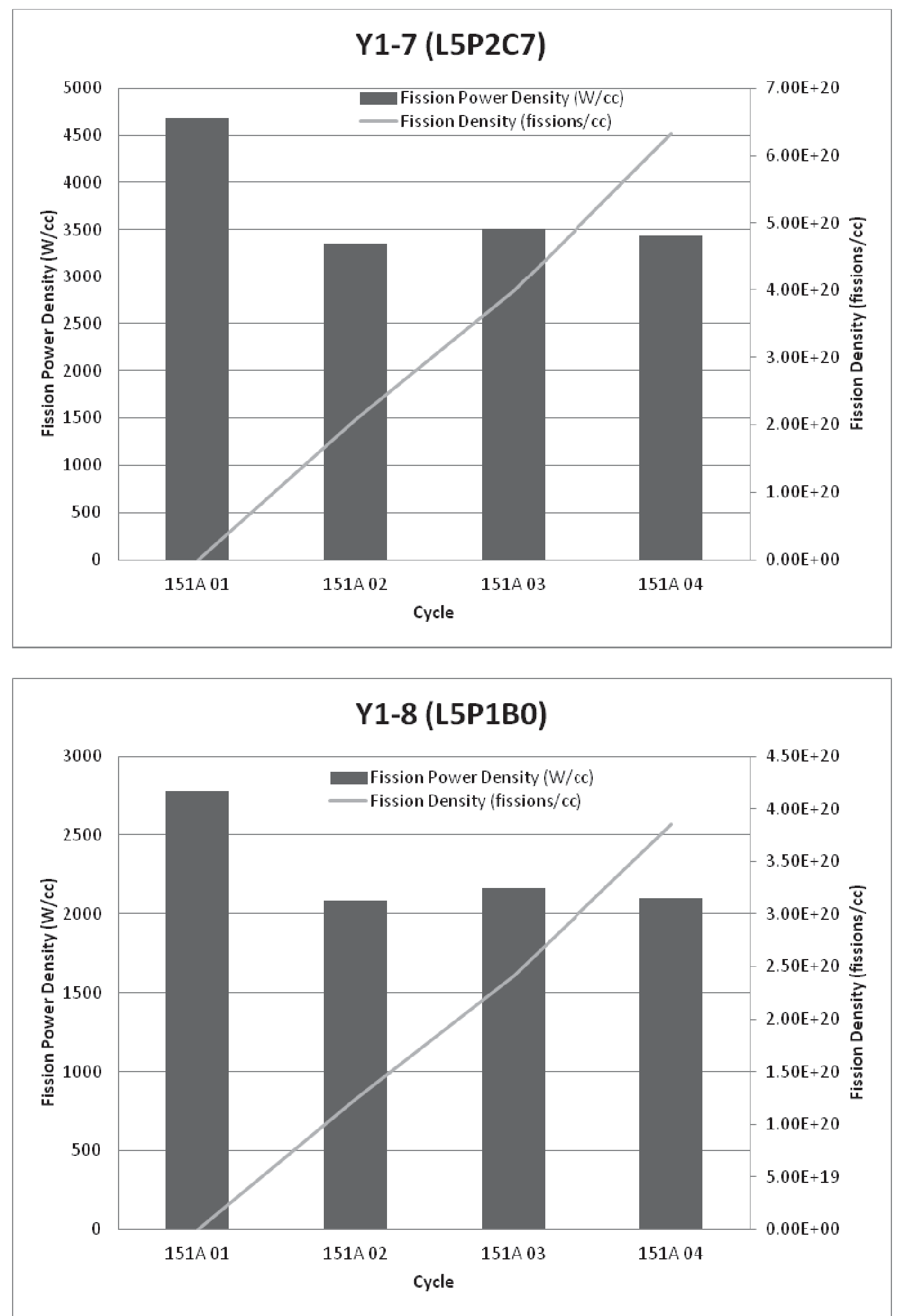


\section{A-3. Capsule Y-2}

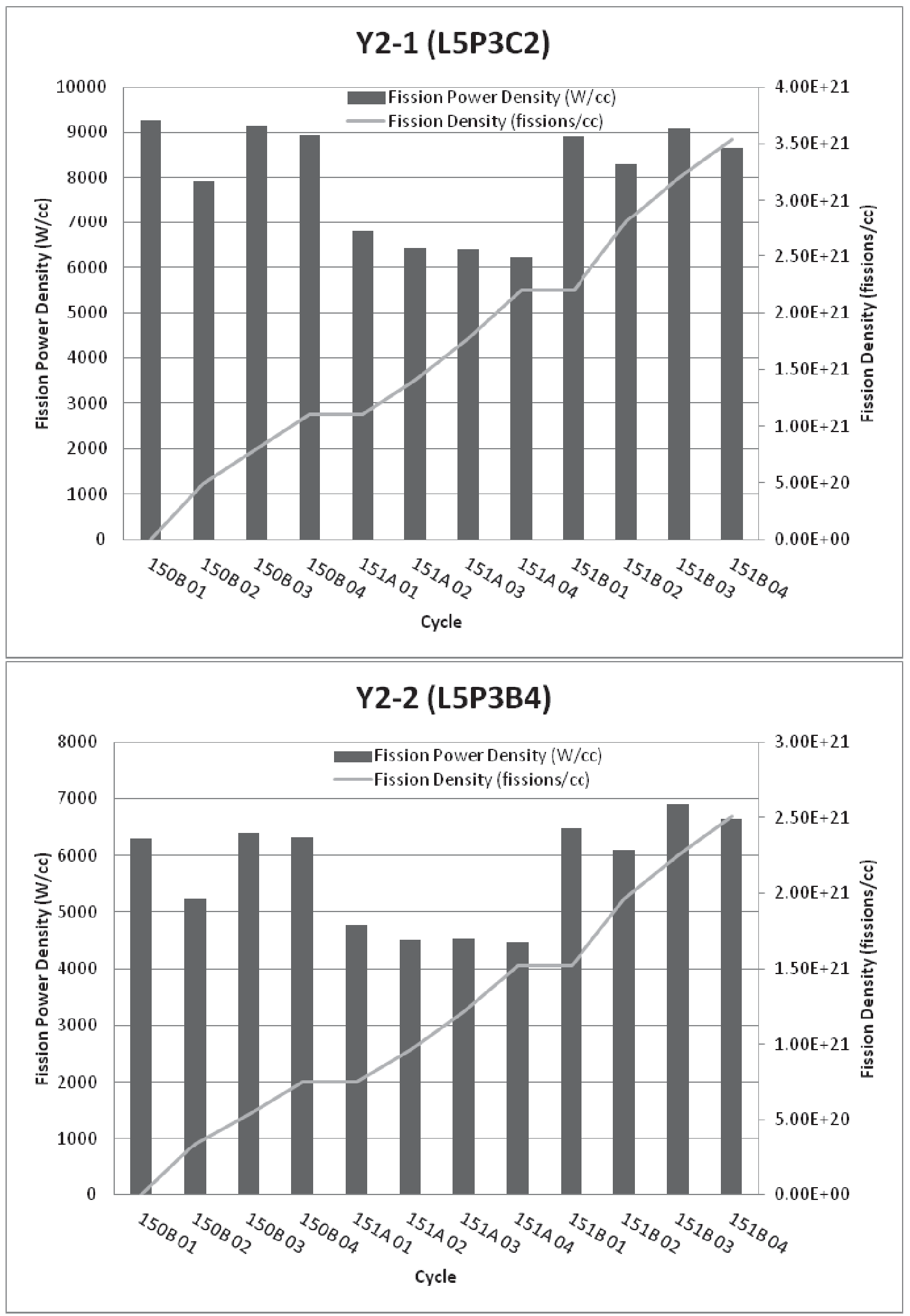



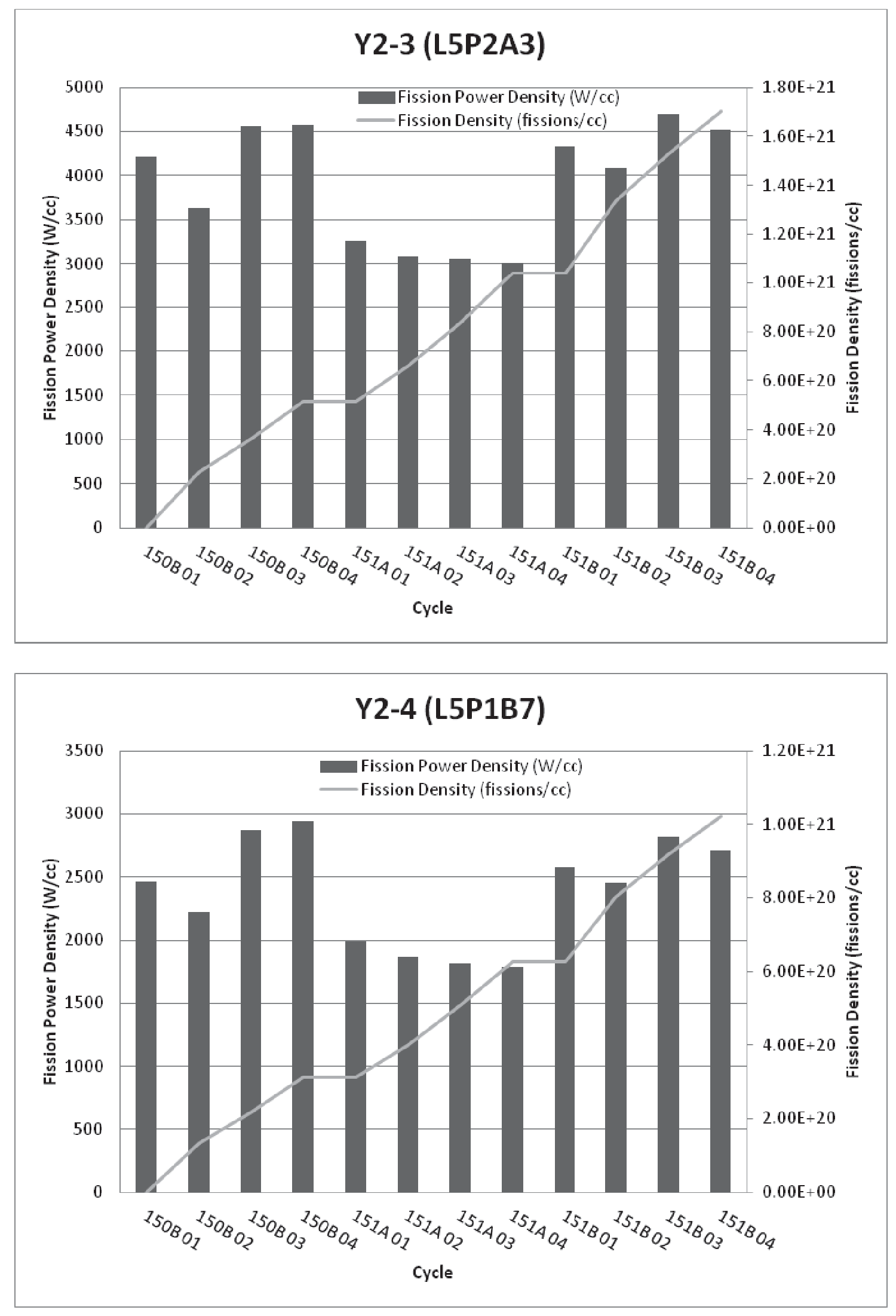

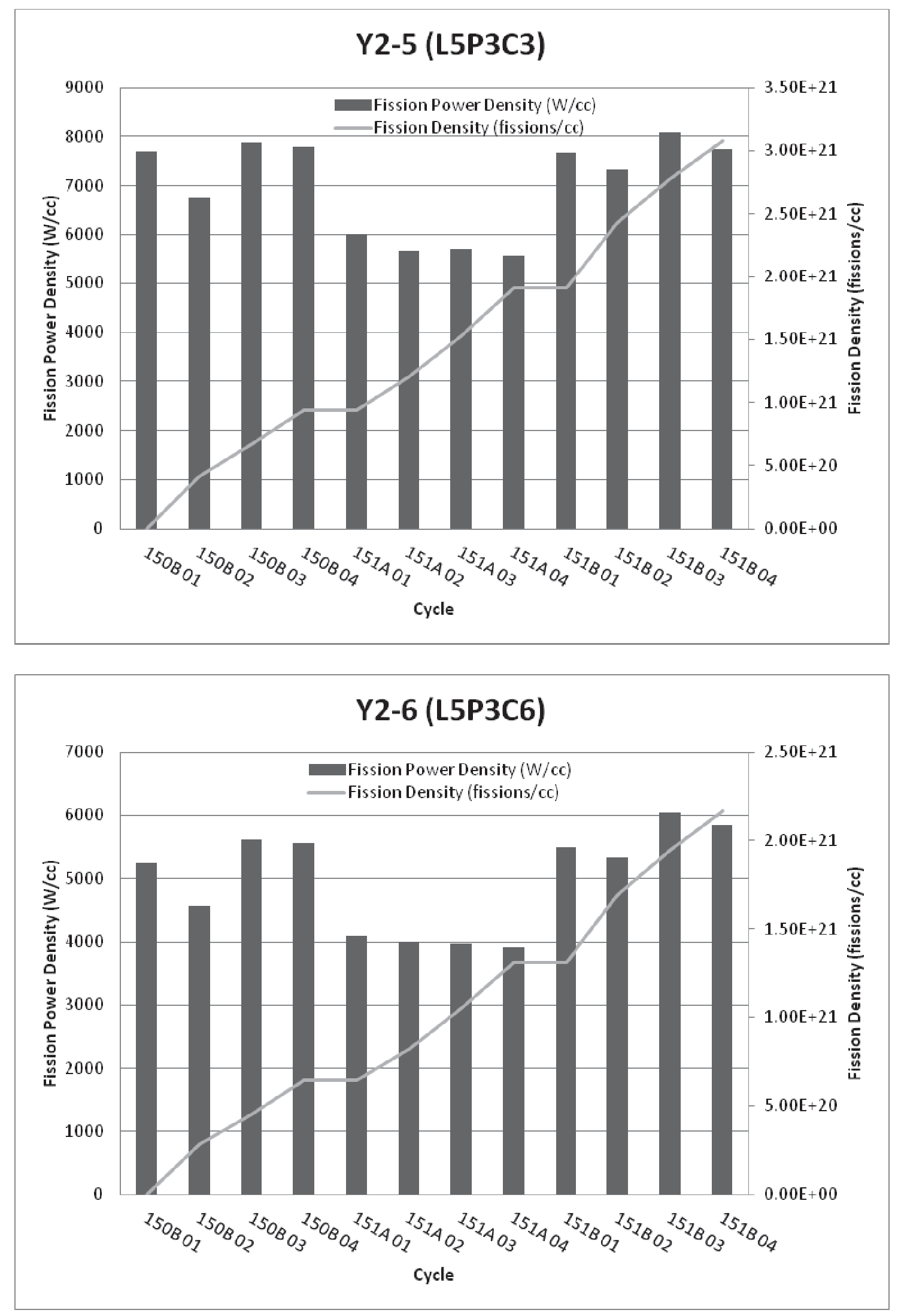

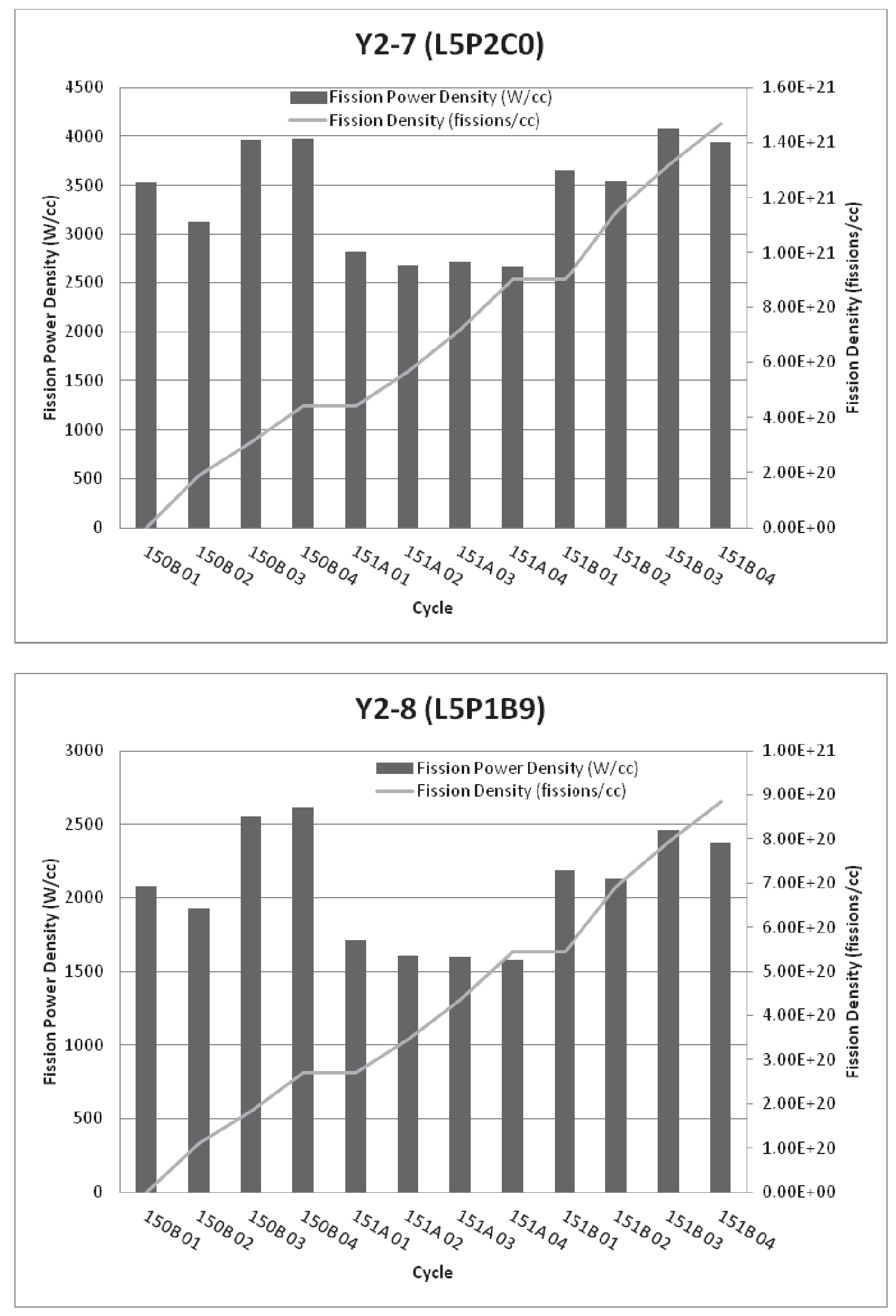


\section{A-4. Capsule Y-3}

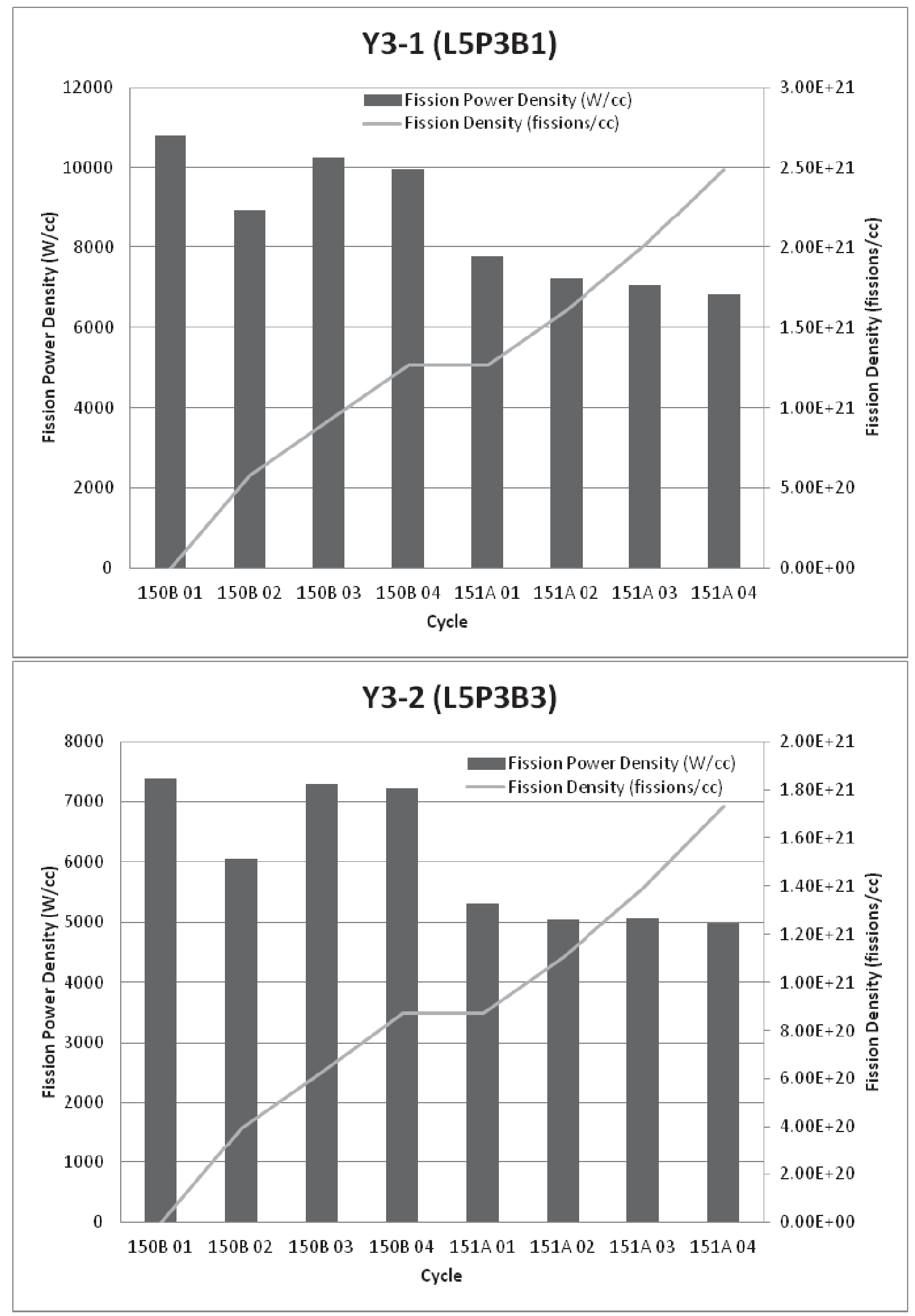



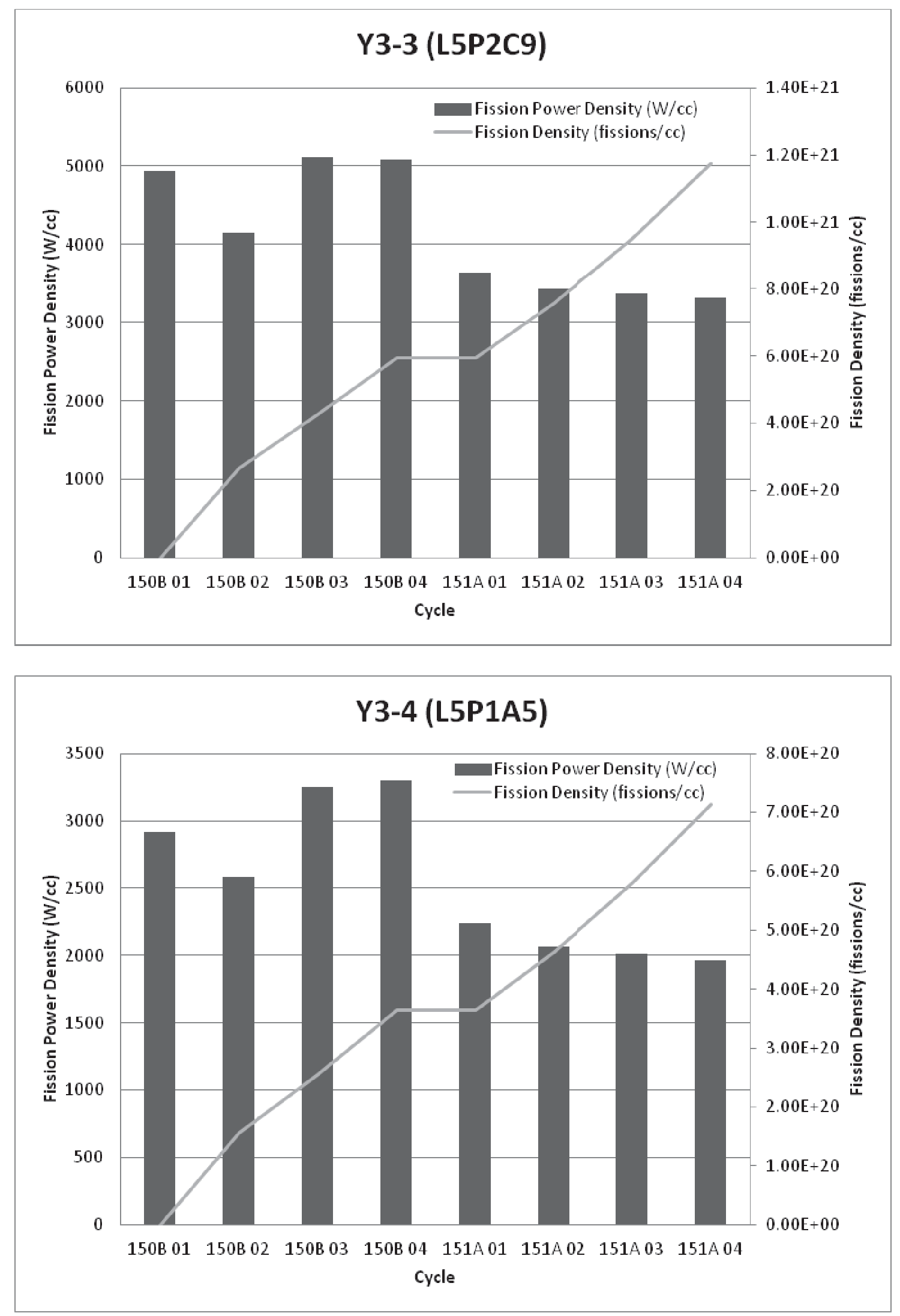

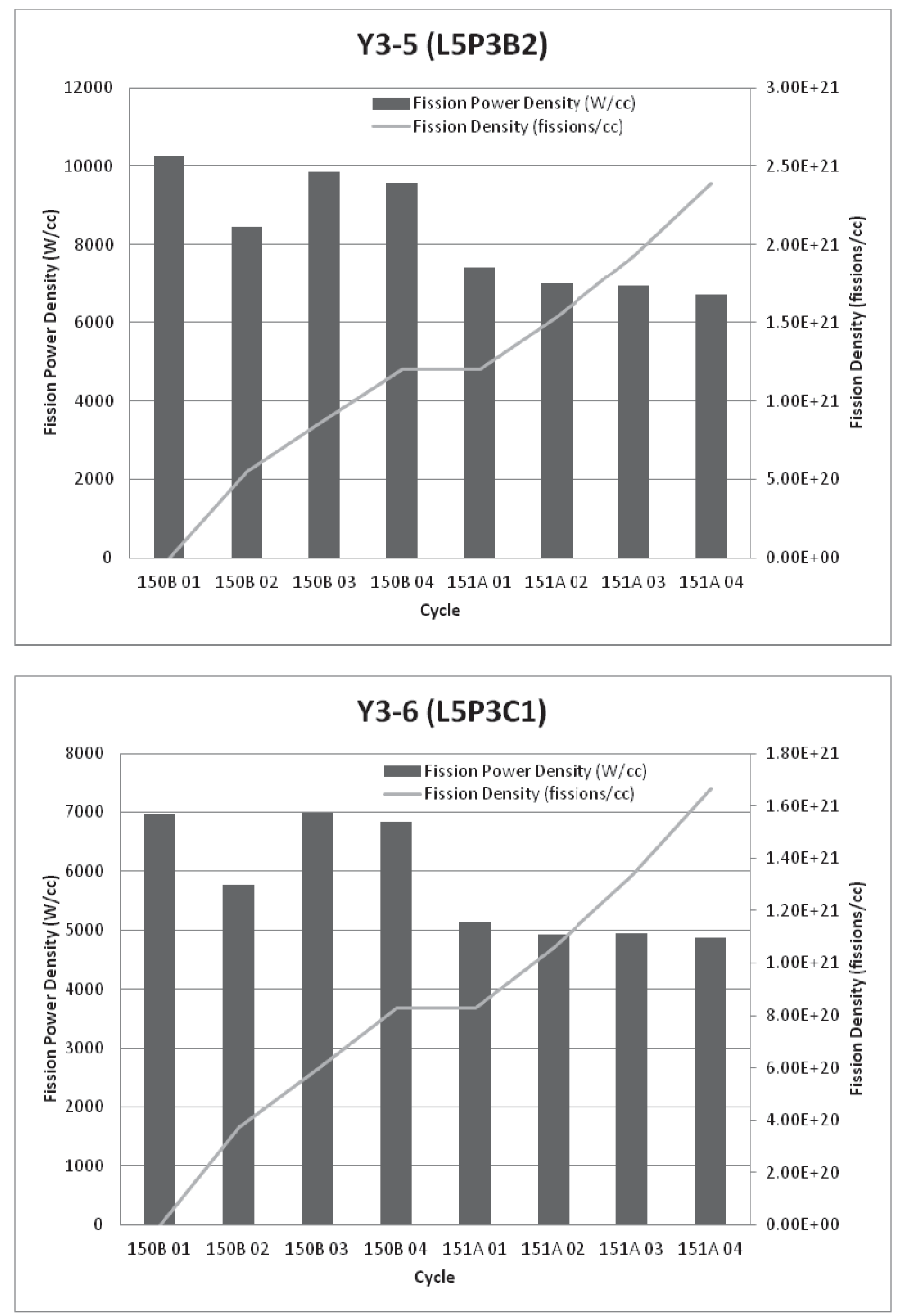

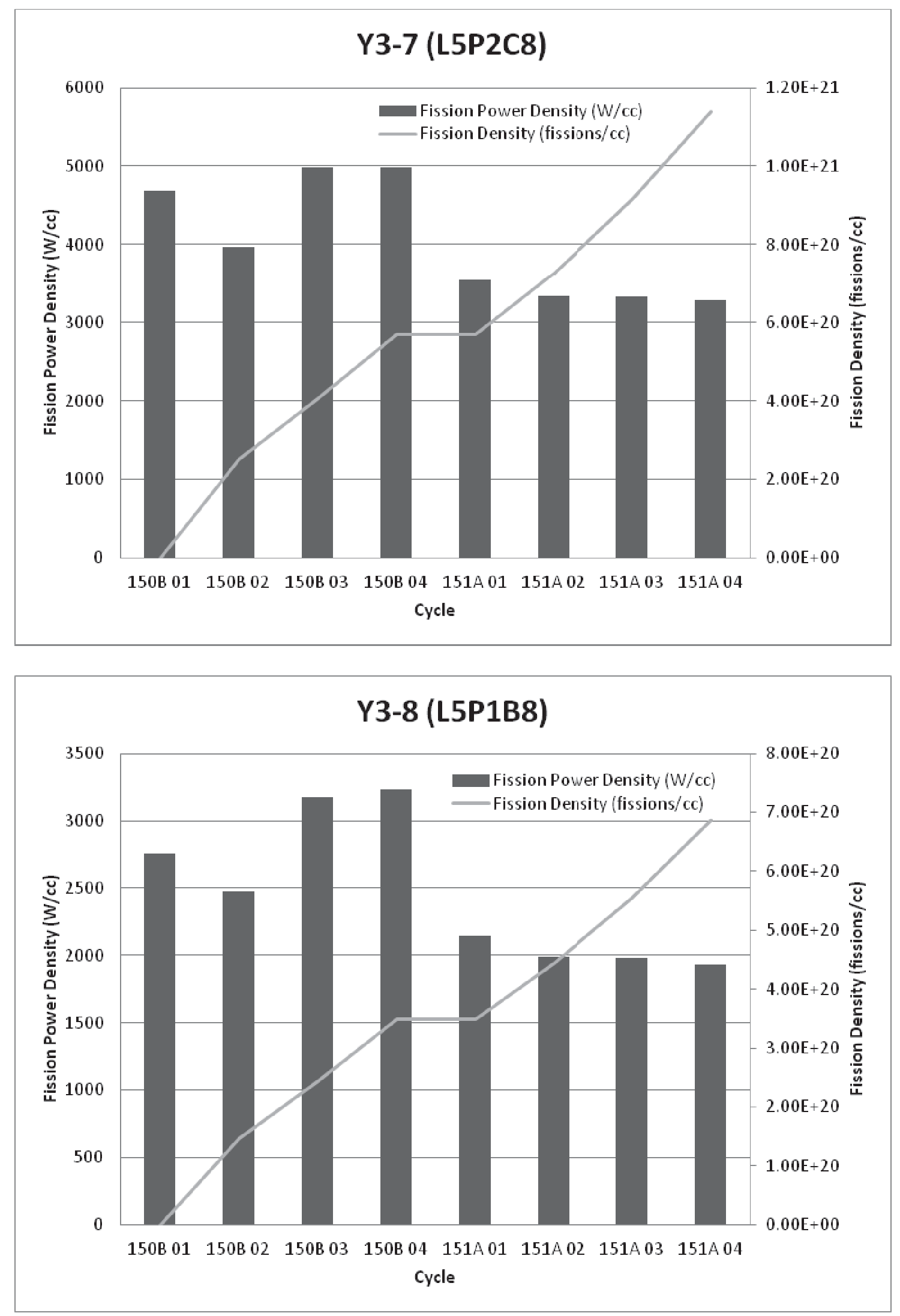
Appendix B

Fission Rate Local to Average Ratio 2D Gradient Maps 


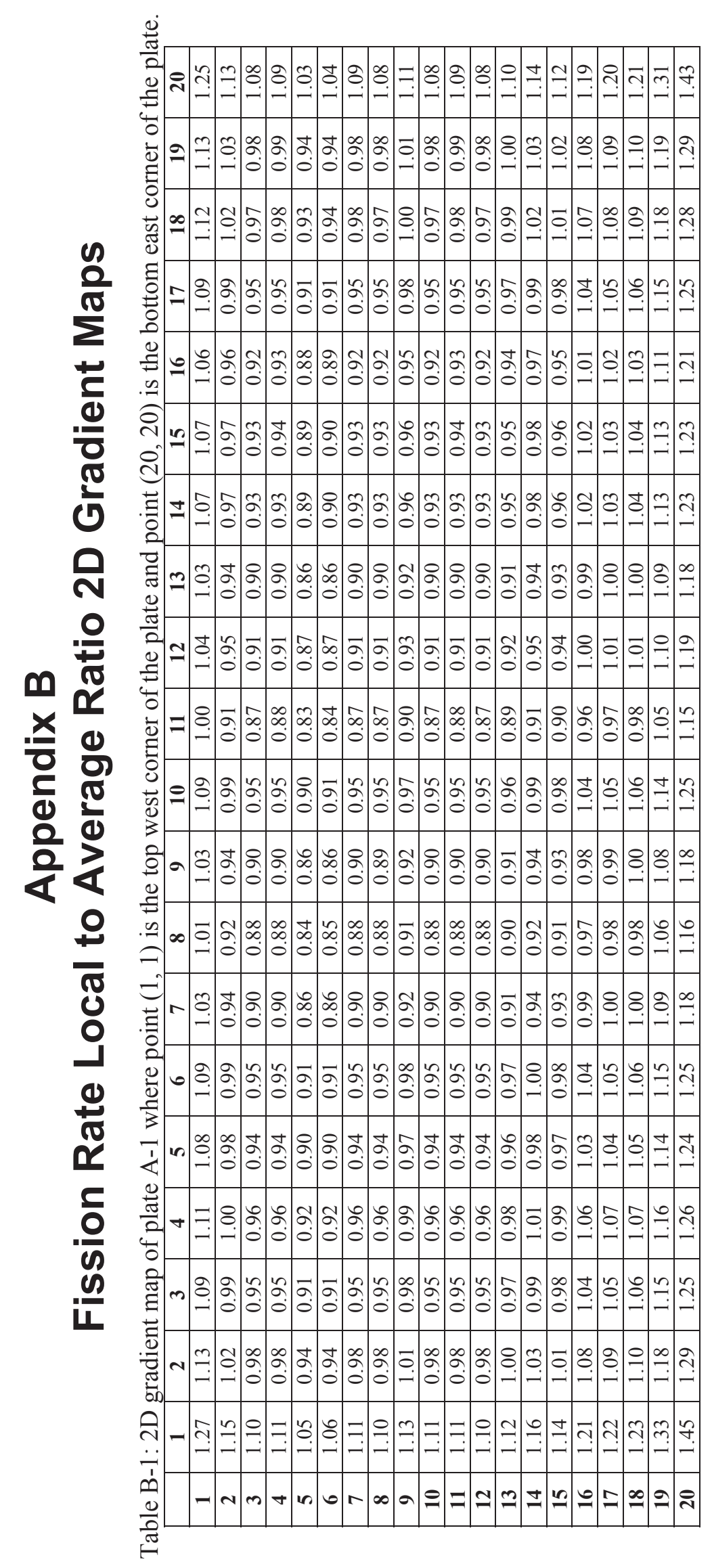




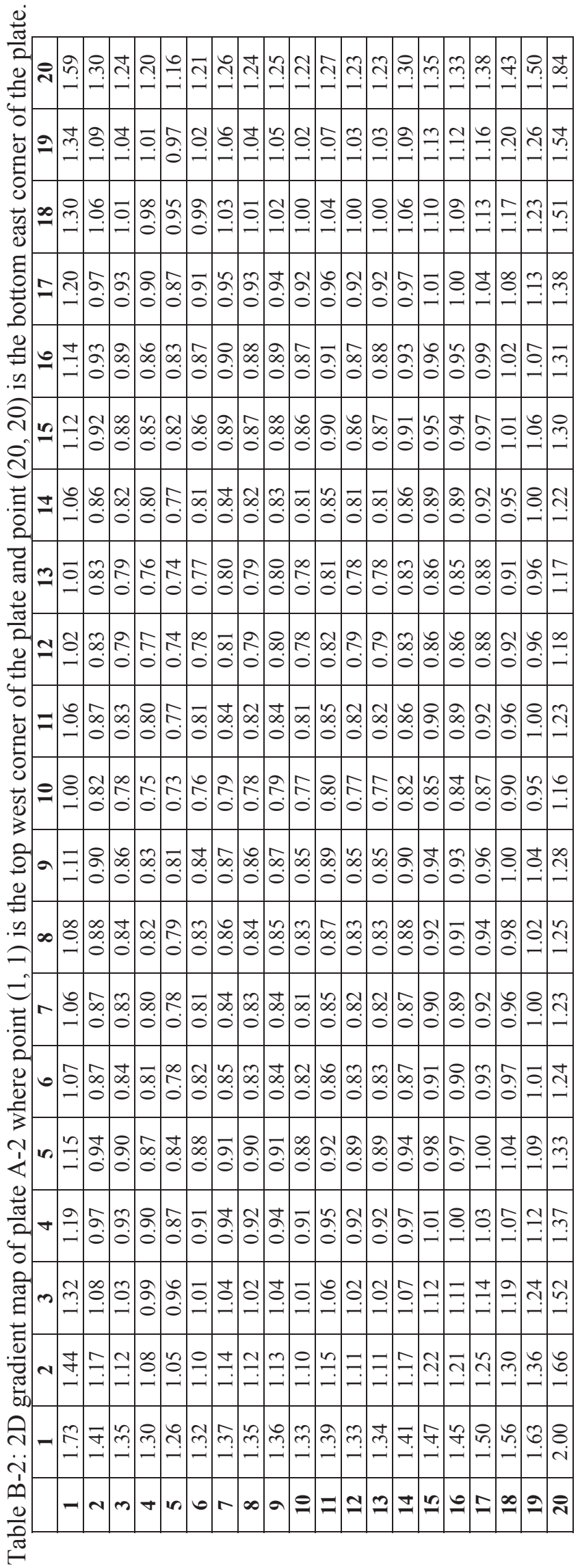

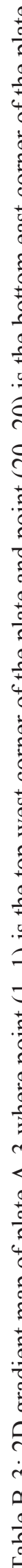

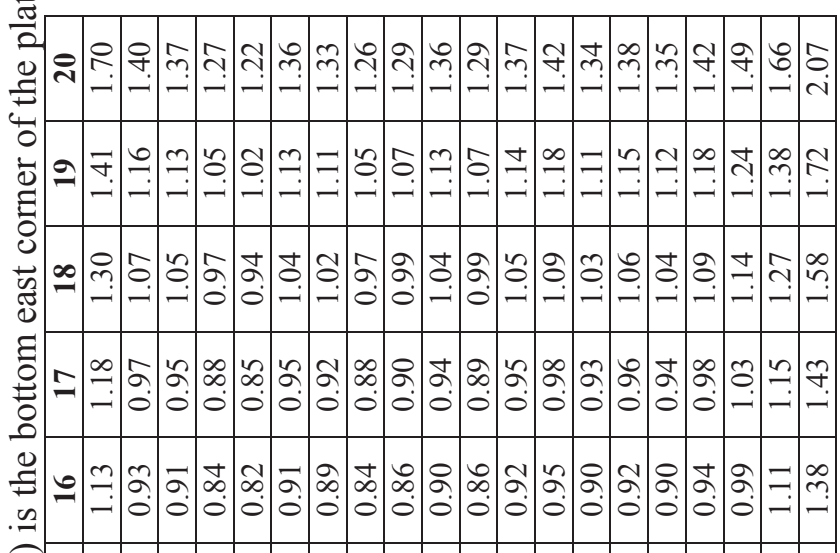
Әิ ঠ)

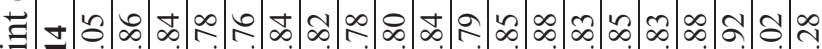

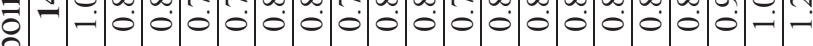

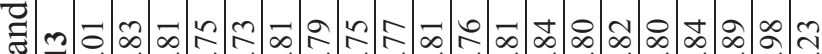

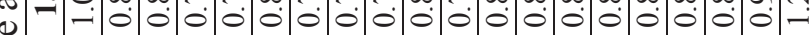
ㄴ.

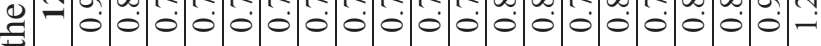

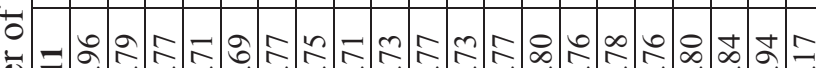

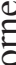

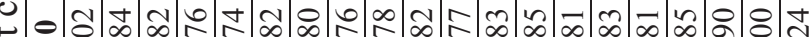

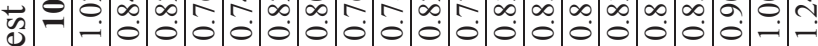

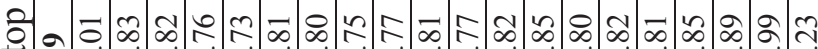

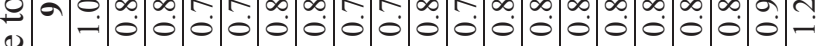

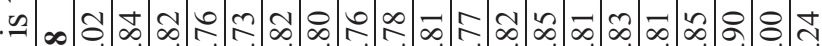

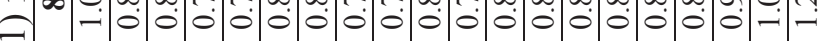
こ

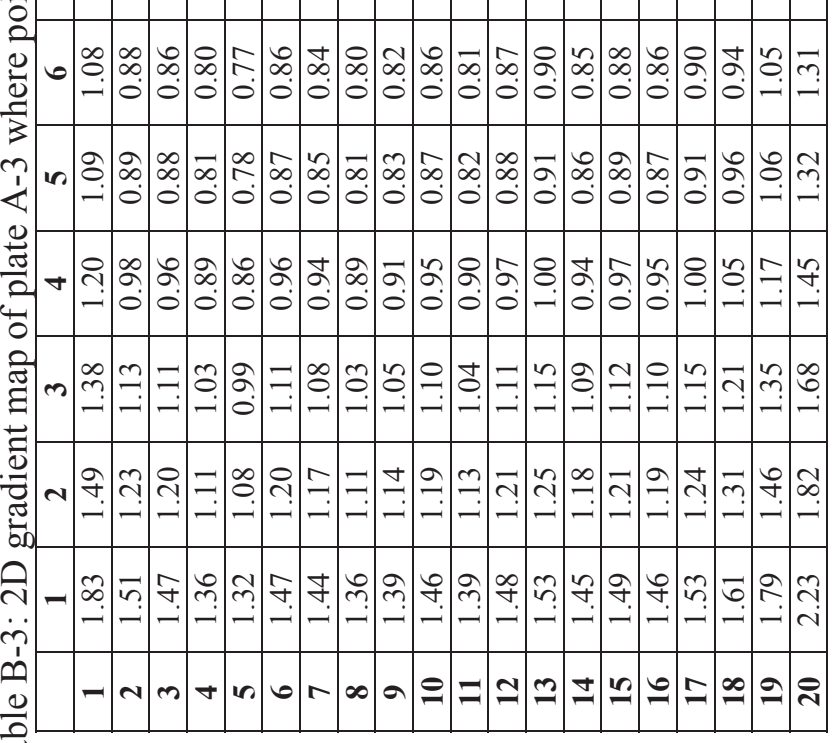



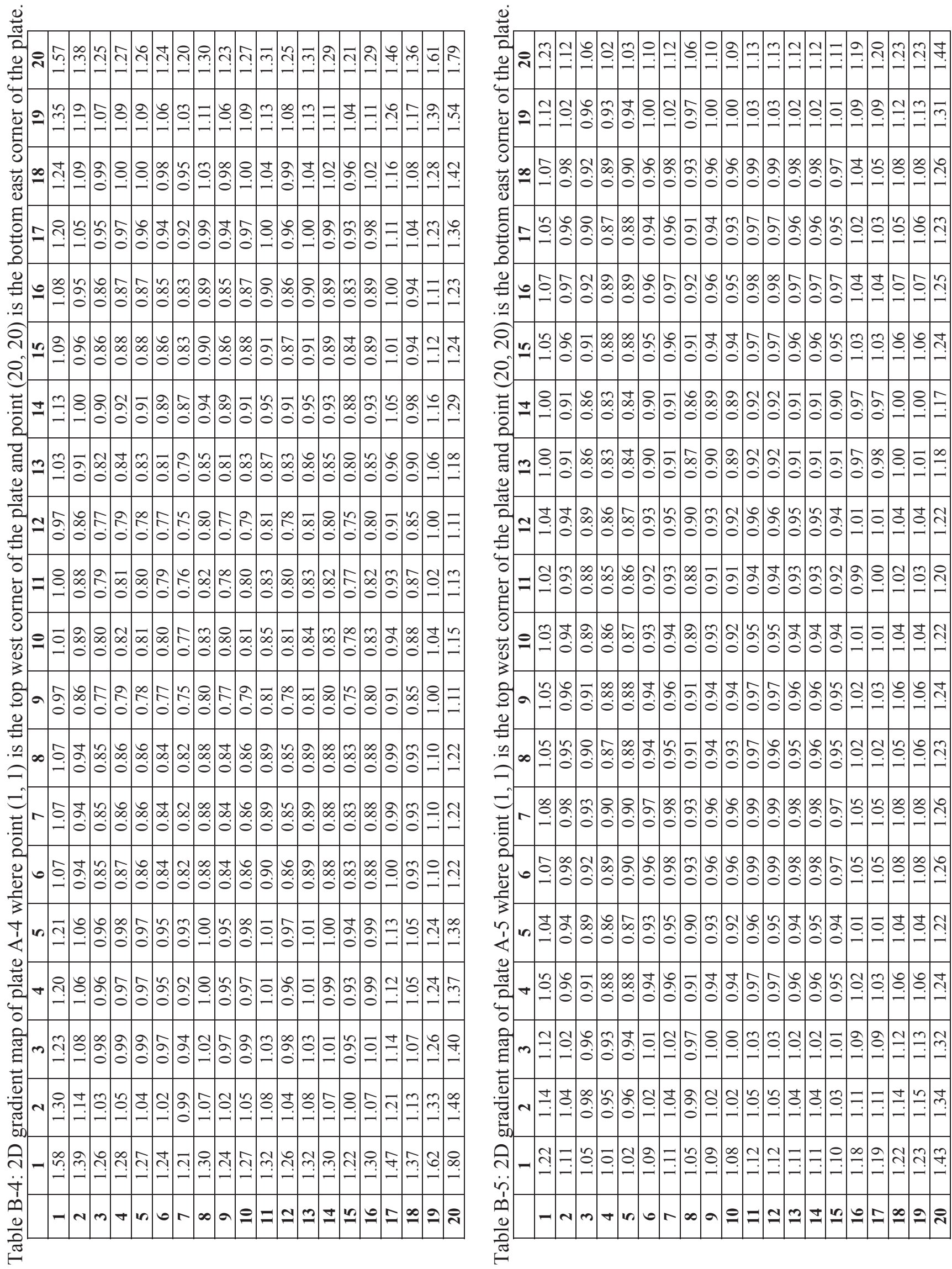

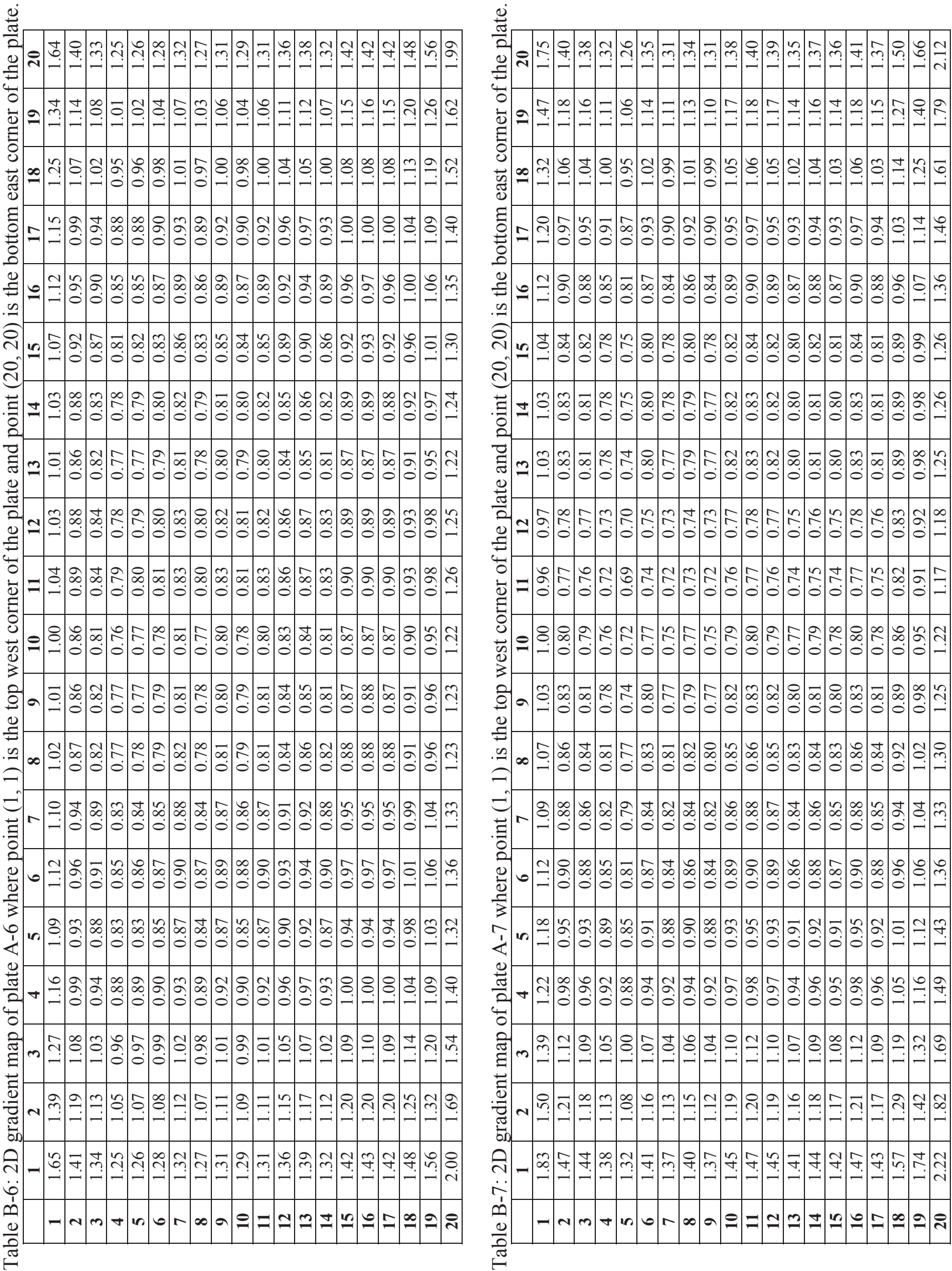

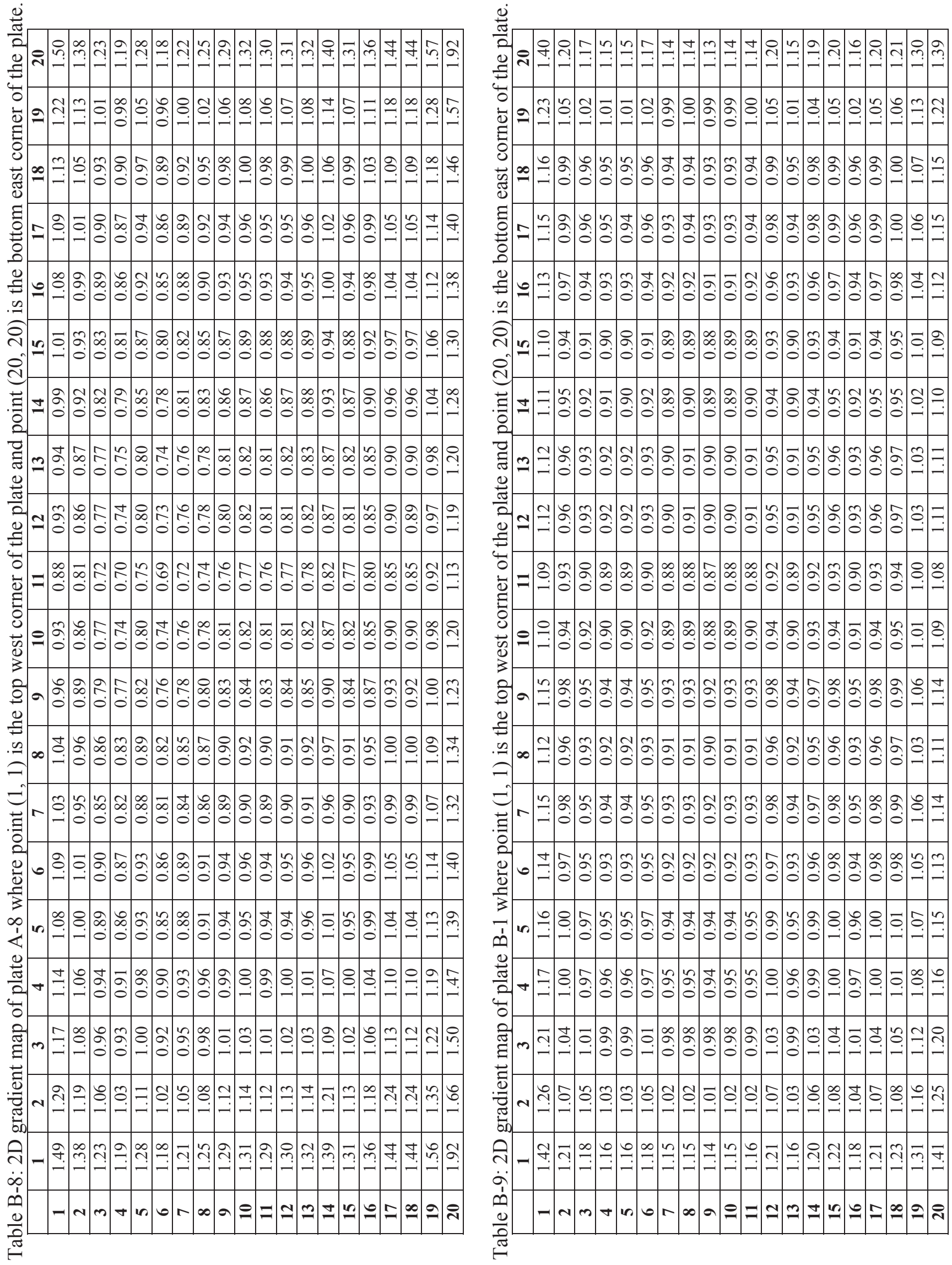


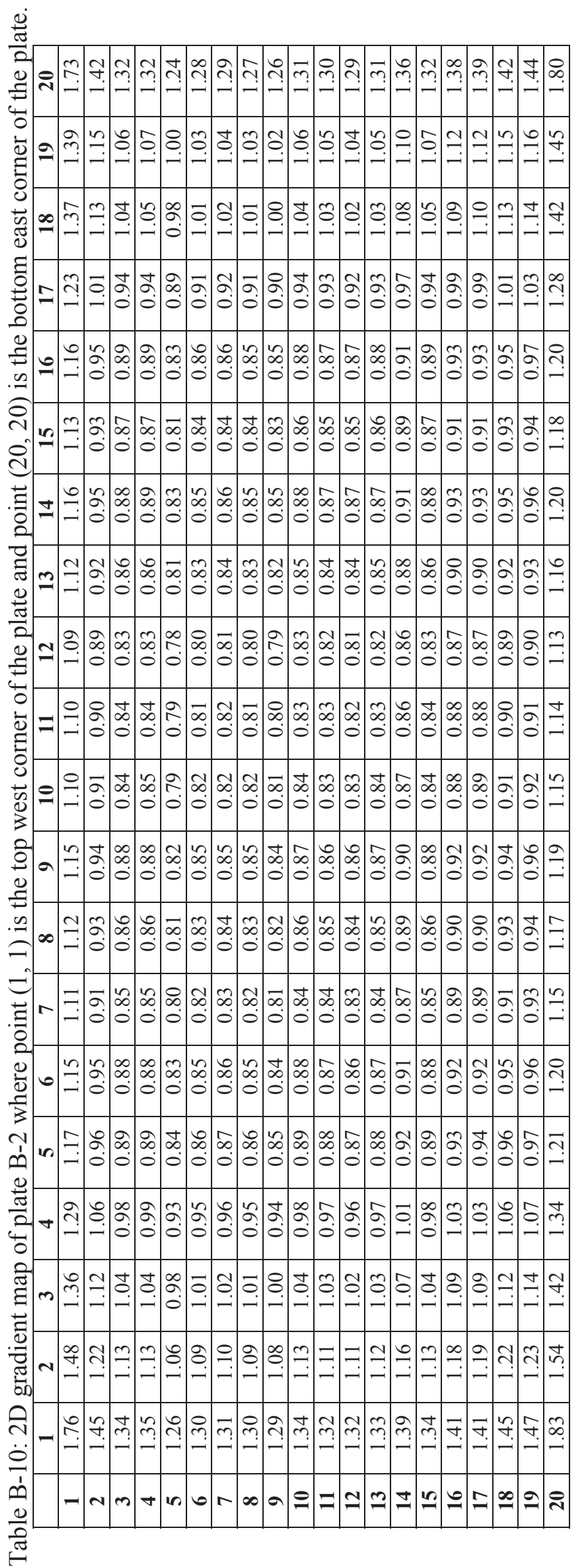

.

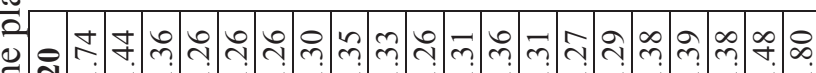
$\Xi$ $\dot{0}$

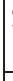

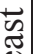
ชิ

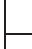

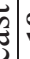

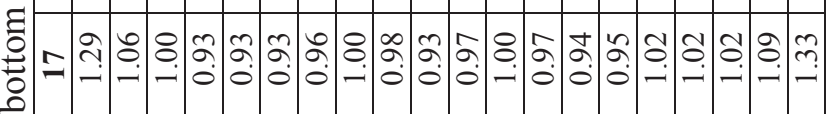
‡

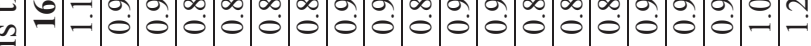

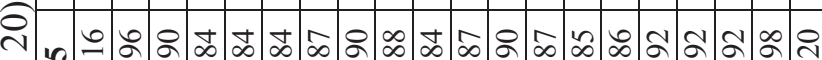

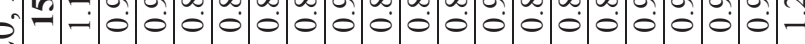

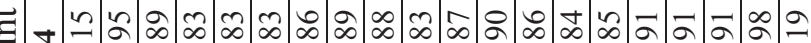

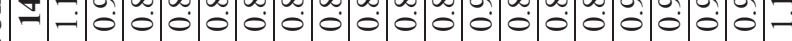

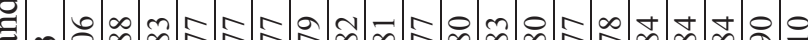

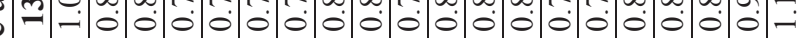
$\frac{\pi}{2}$

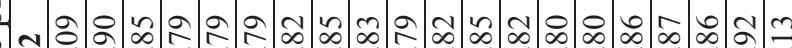

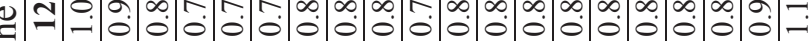

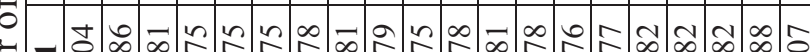

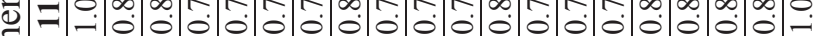

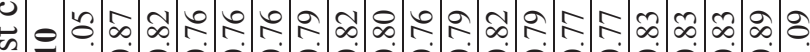

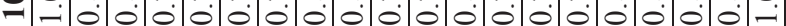

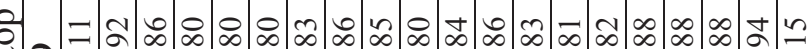

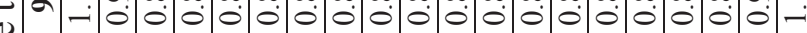

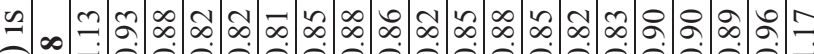

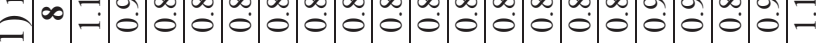
تص

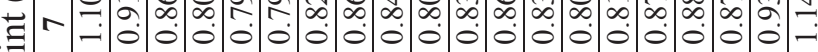

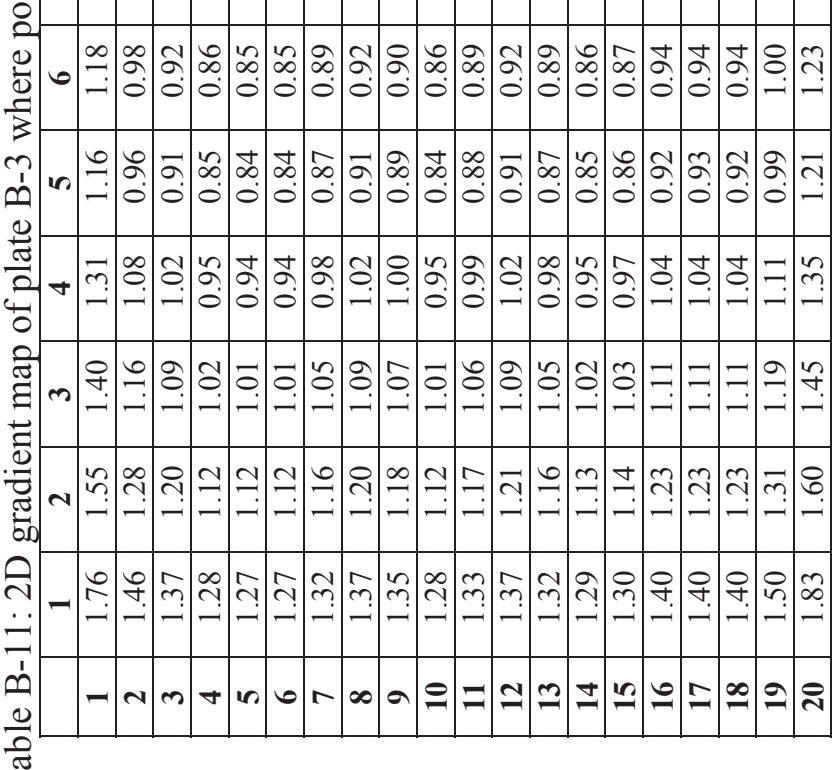




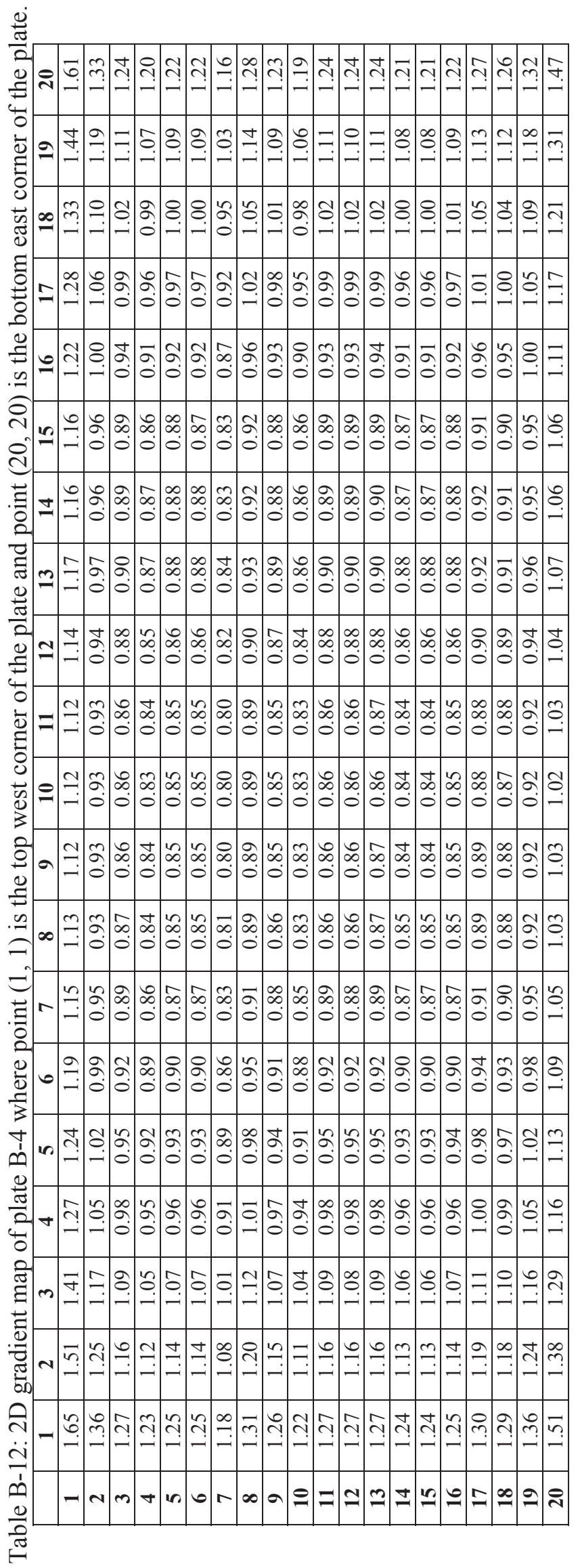
告

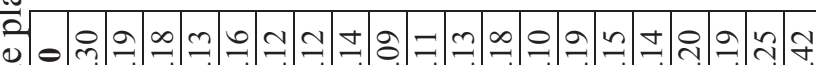

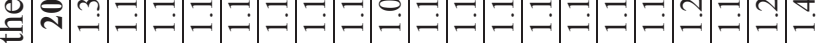

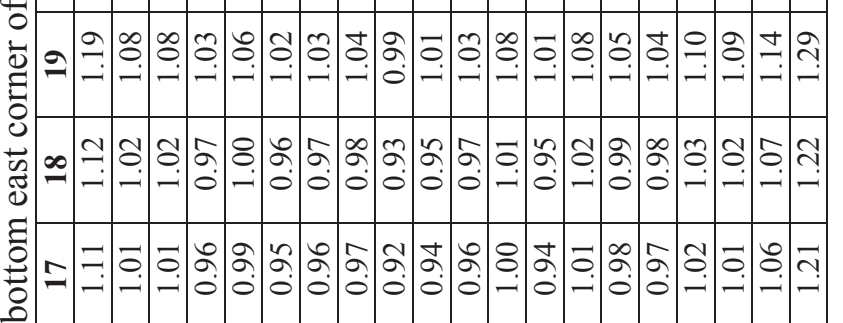

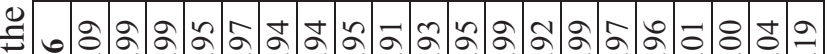
-

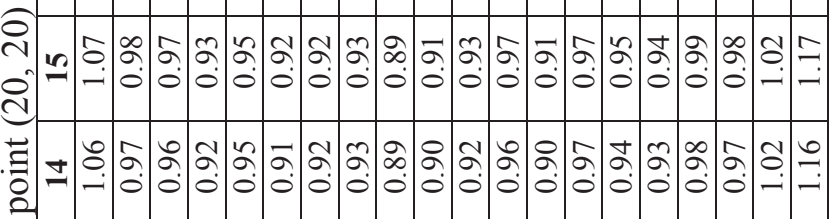

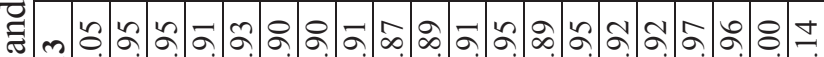

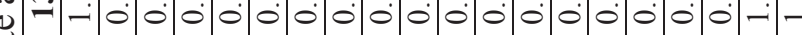

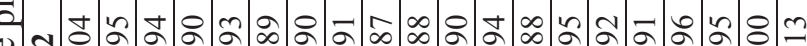

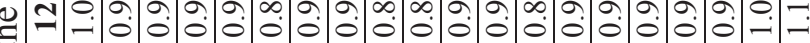

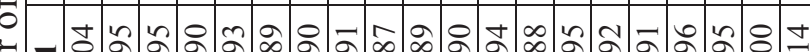

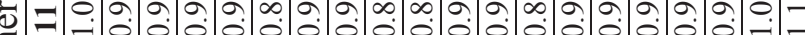

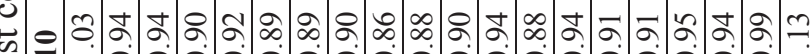

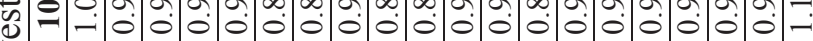

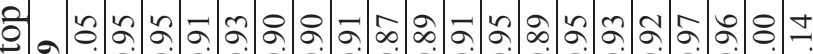

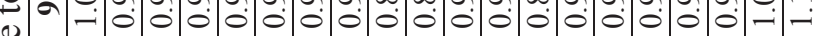

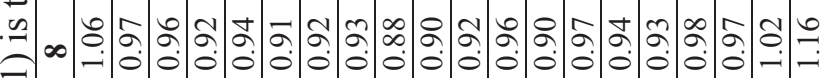

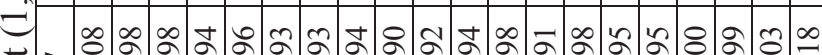
节

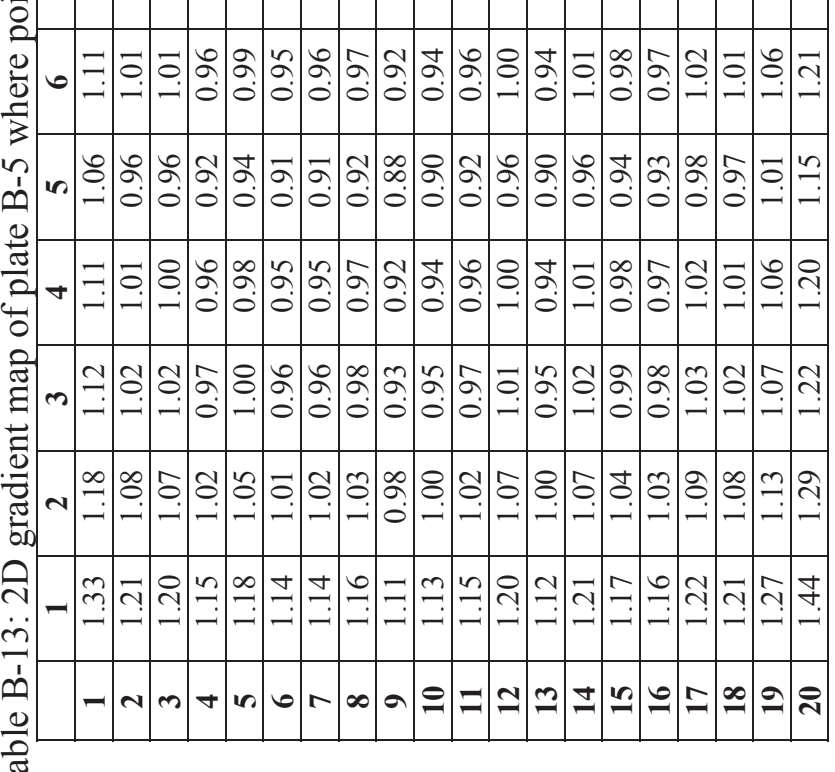




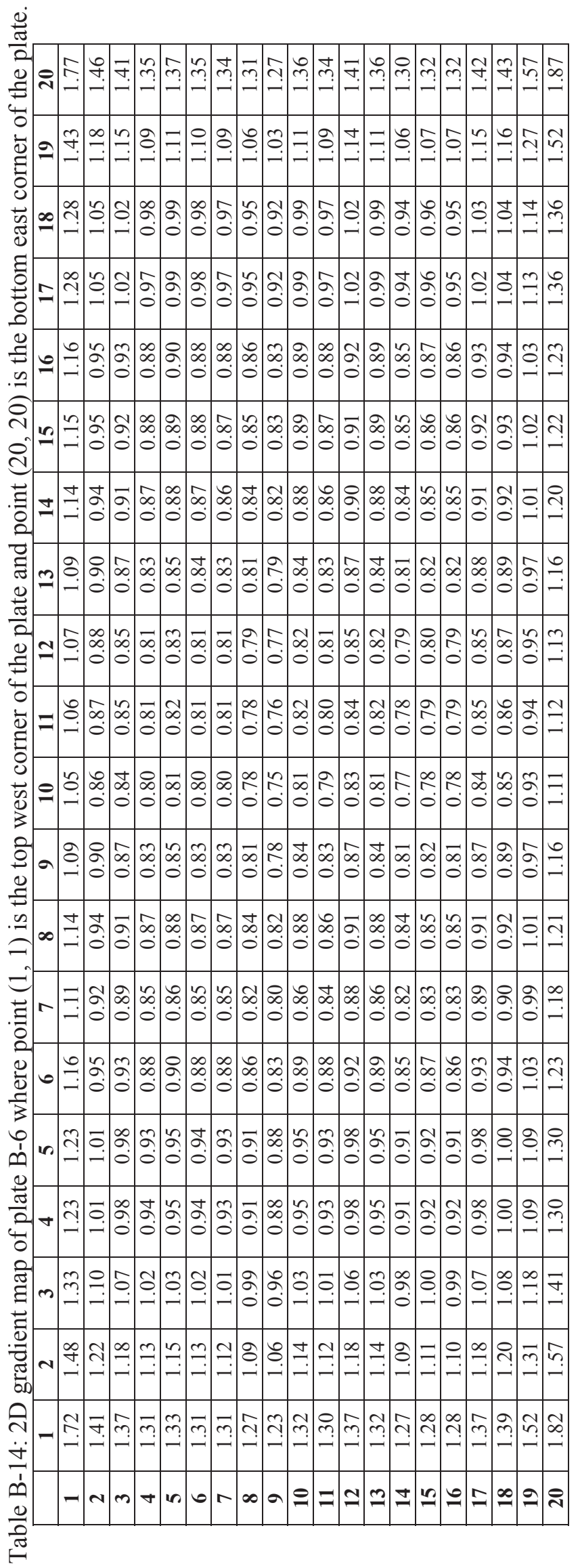

.

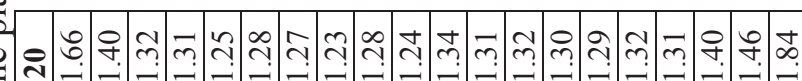

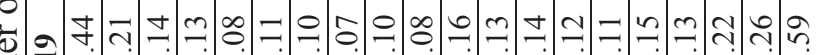
ठํำ

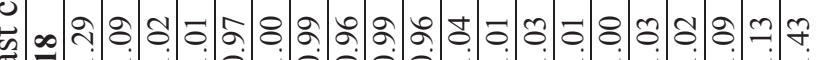

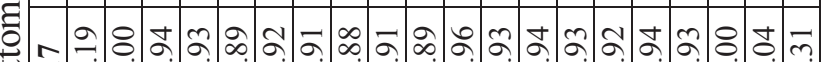

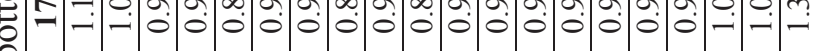

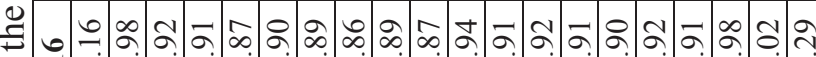

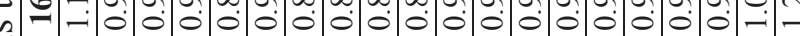
ิิm

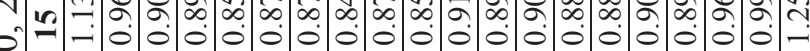

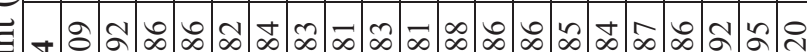

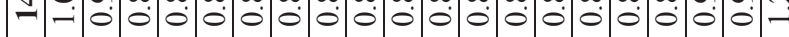

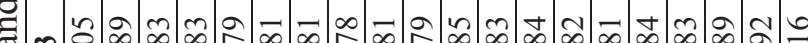

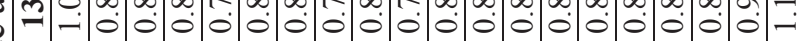

$\frac{\pi}{2}$ ง

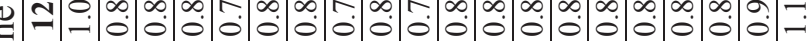

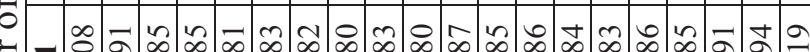

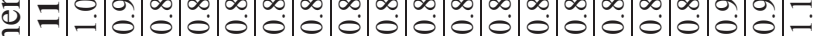

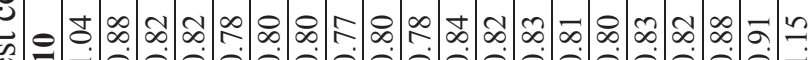

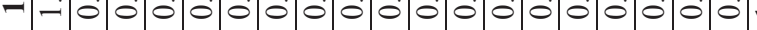
ว ๖ ఫ a ح $\infty$ ప.

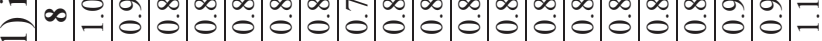

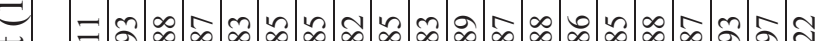

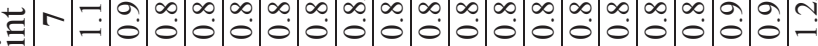
2

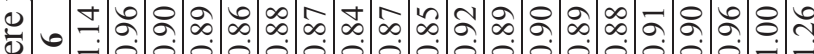
๑

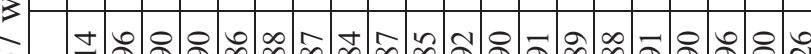
向 nn =:

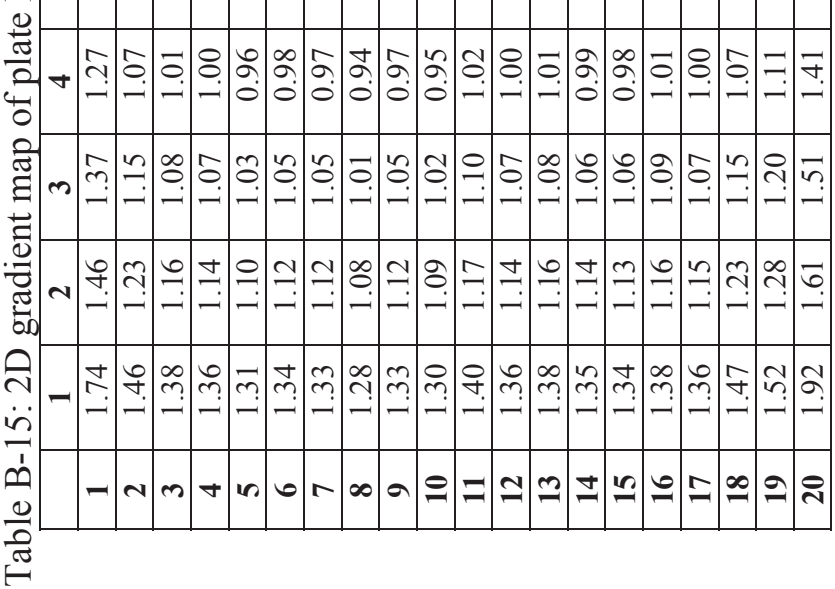




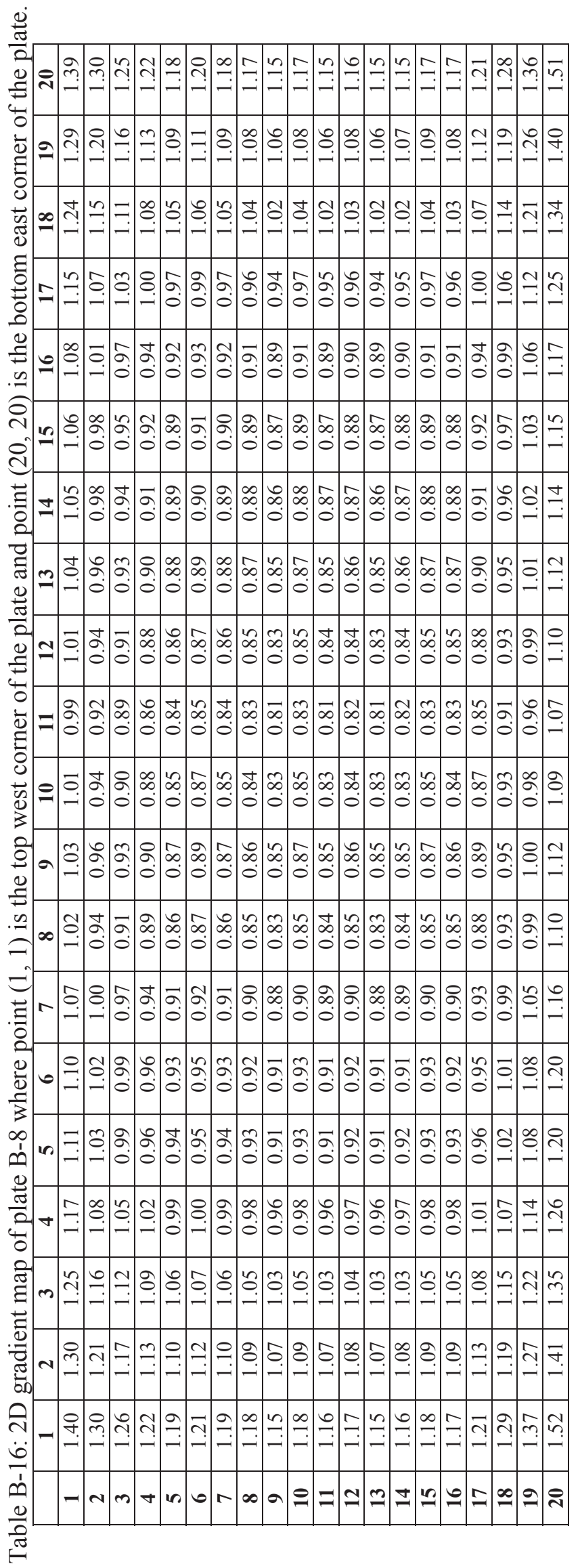

.

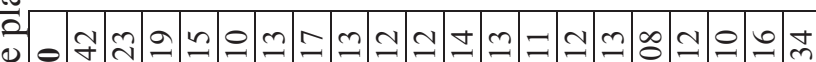
그 r. ๑ี $=$ ำ ¿

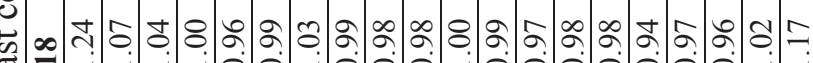

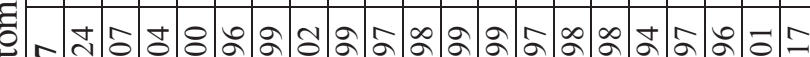

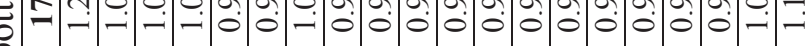

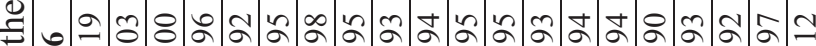

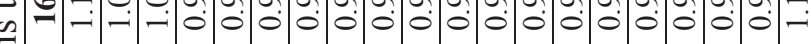

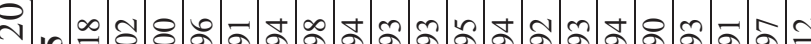
ஊ ت̇ オ

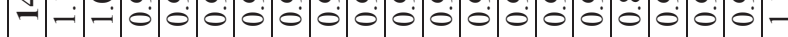

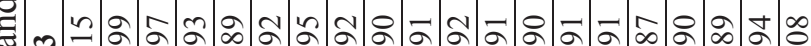

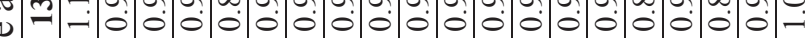
$\frac{\pi}{2}$

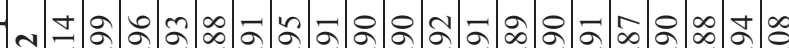

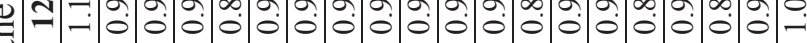

-

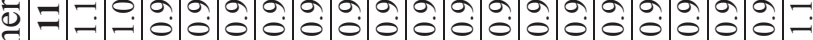

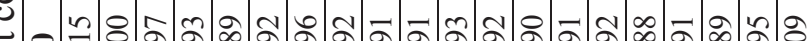

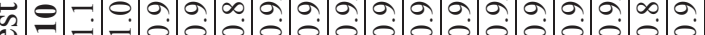
3

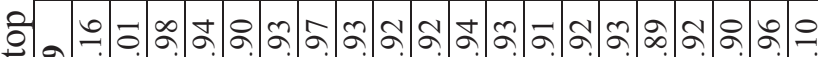
๘

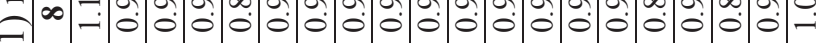
Inc.m.

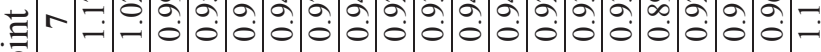

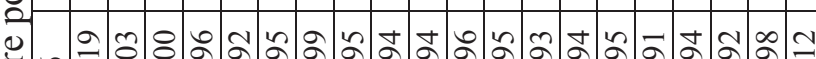
๑) ส Ú n

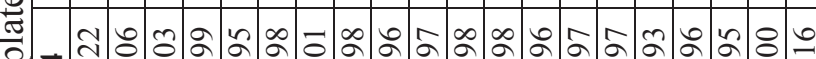

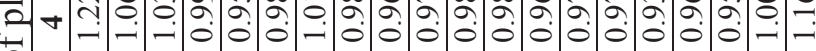

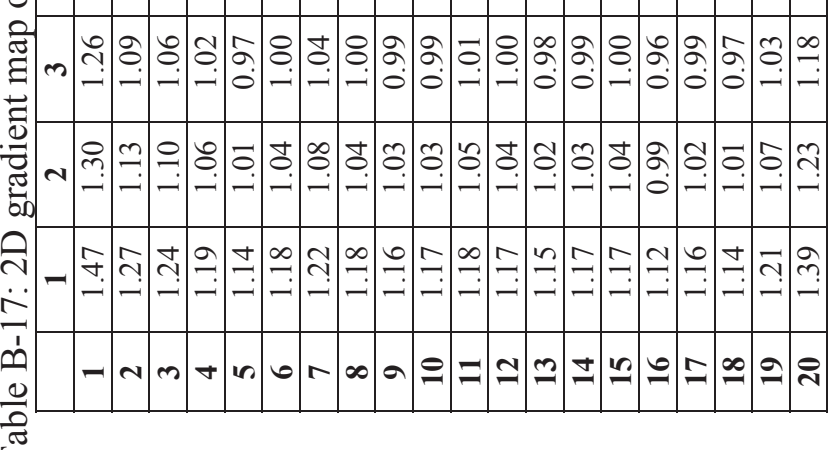




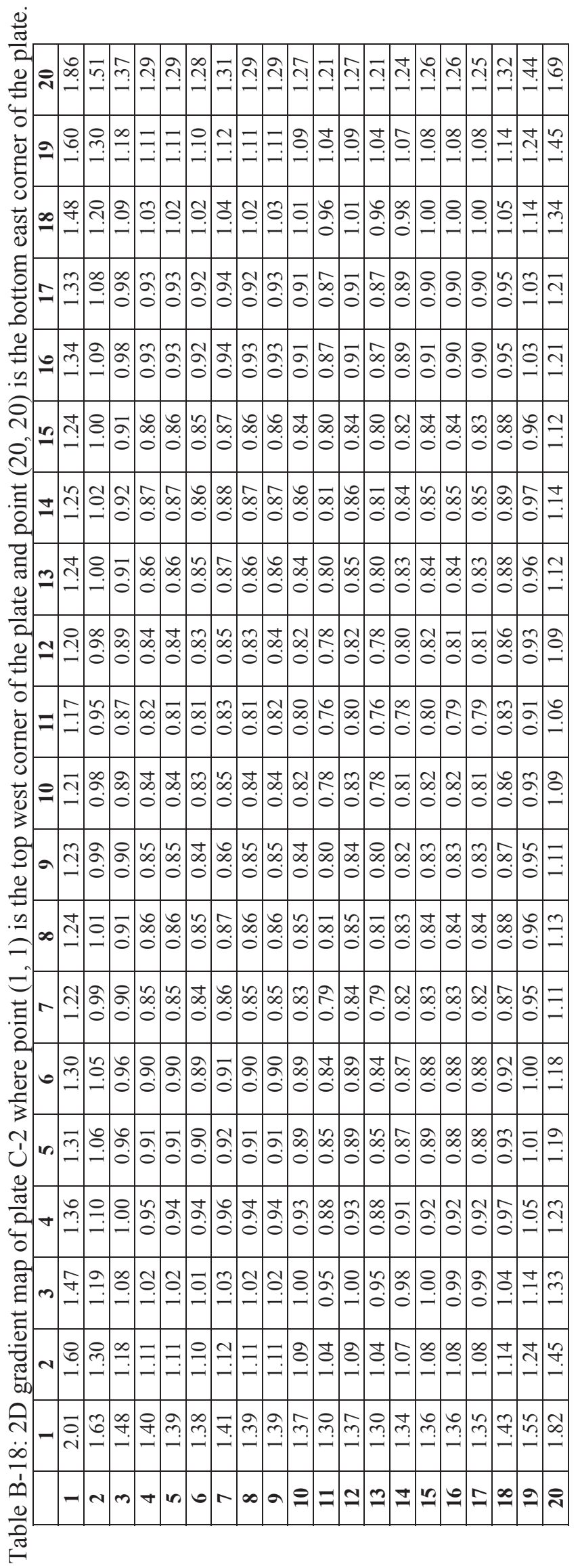

. 幽

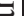

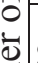
西 $\overrightarrow{\widetilde{E}}$ $\infty$ กิๆ

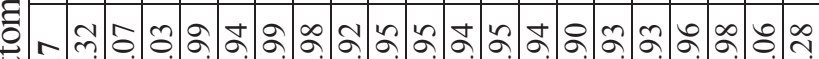
$=-\dot{0} 0.000 .00$

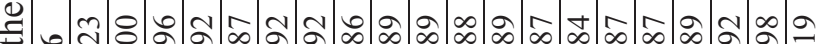

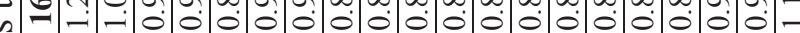
ง

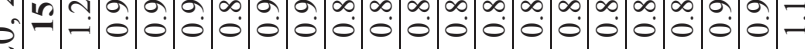

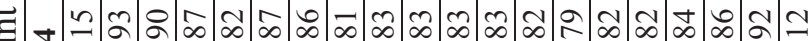

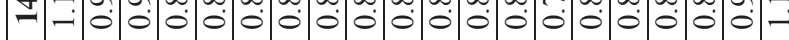

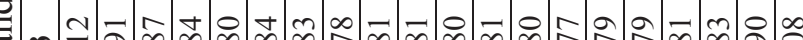

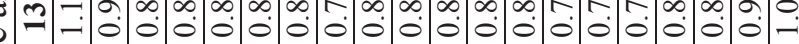

(1)

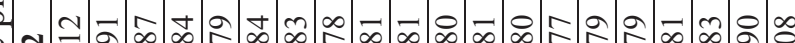

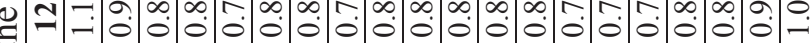

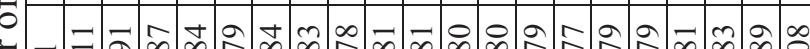

$=\mp \cdot \infty . \infty$

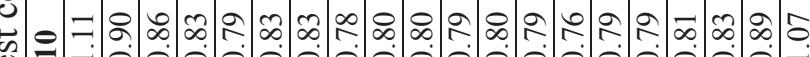

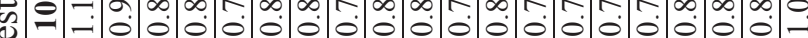
3

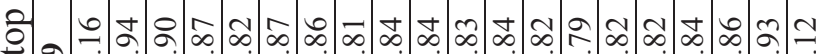

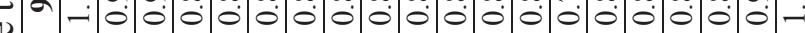

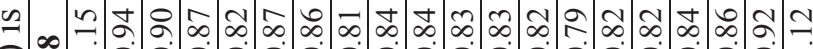

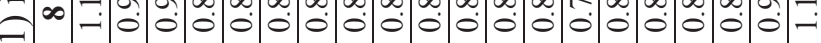

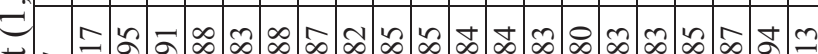

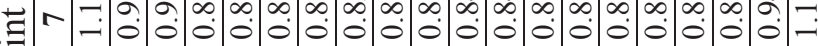

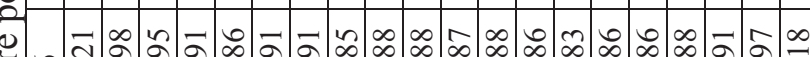
可 $m$ t

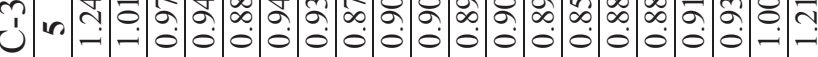

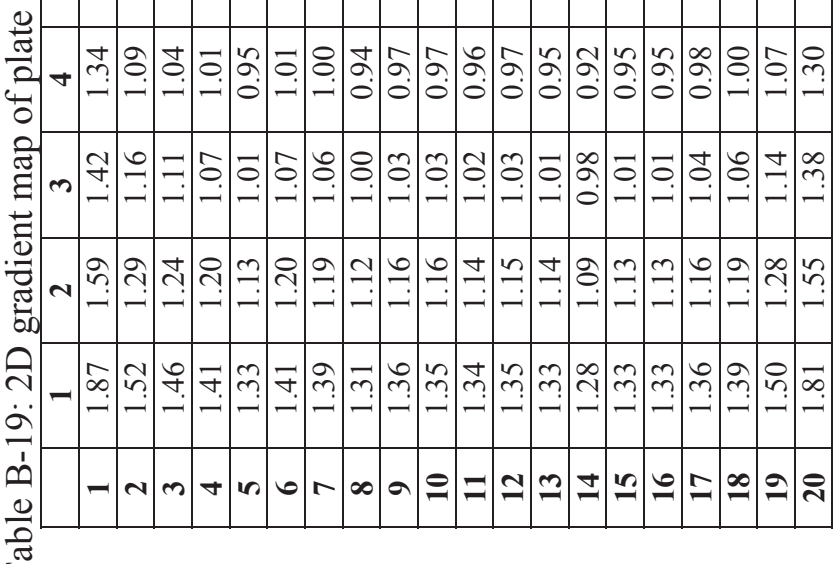



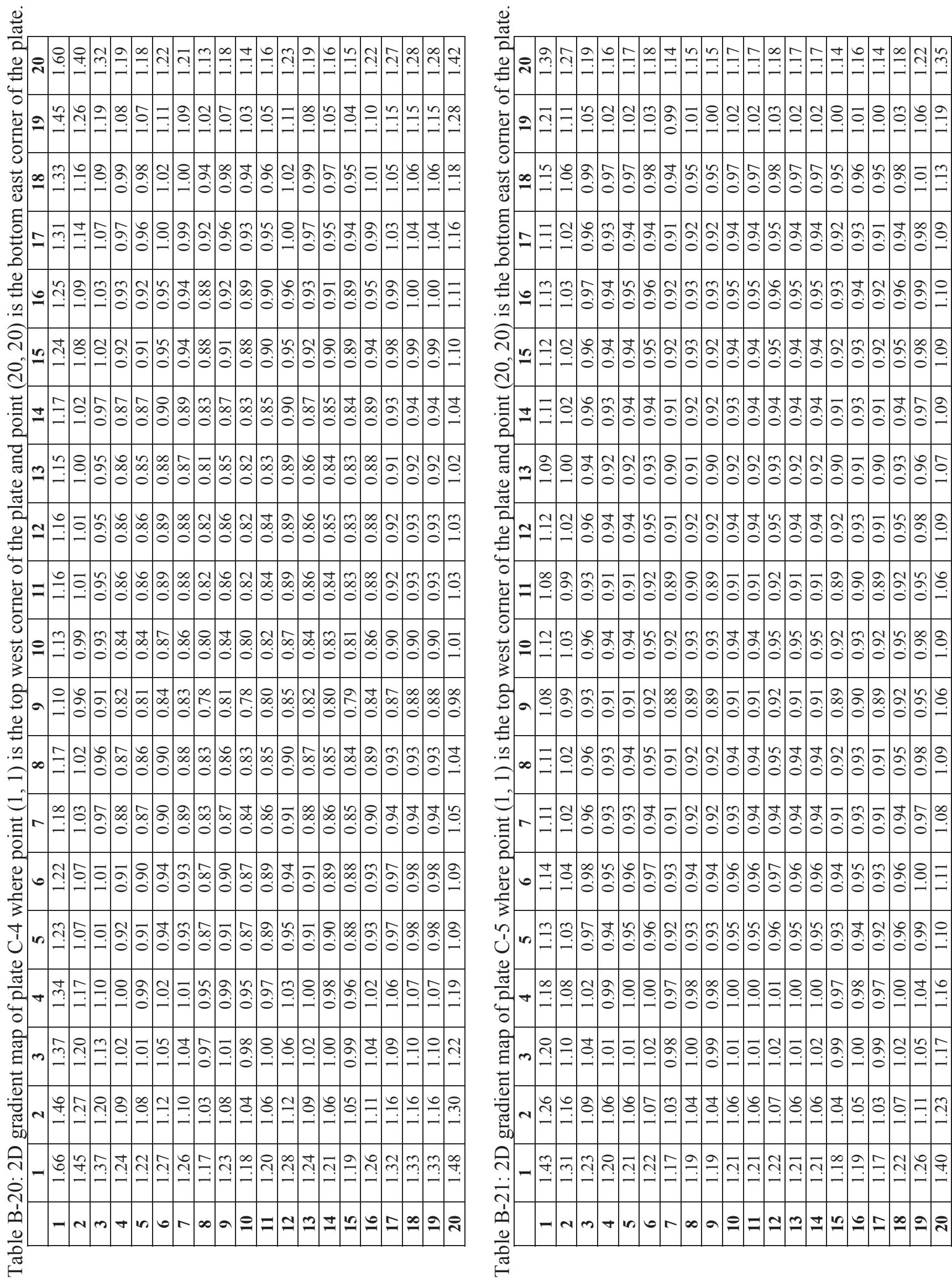


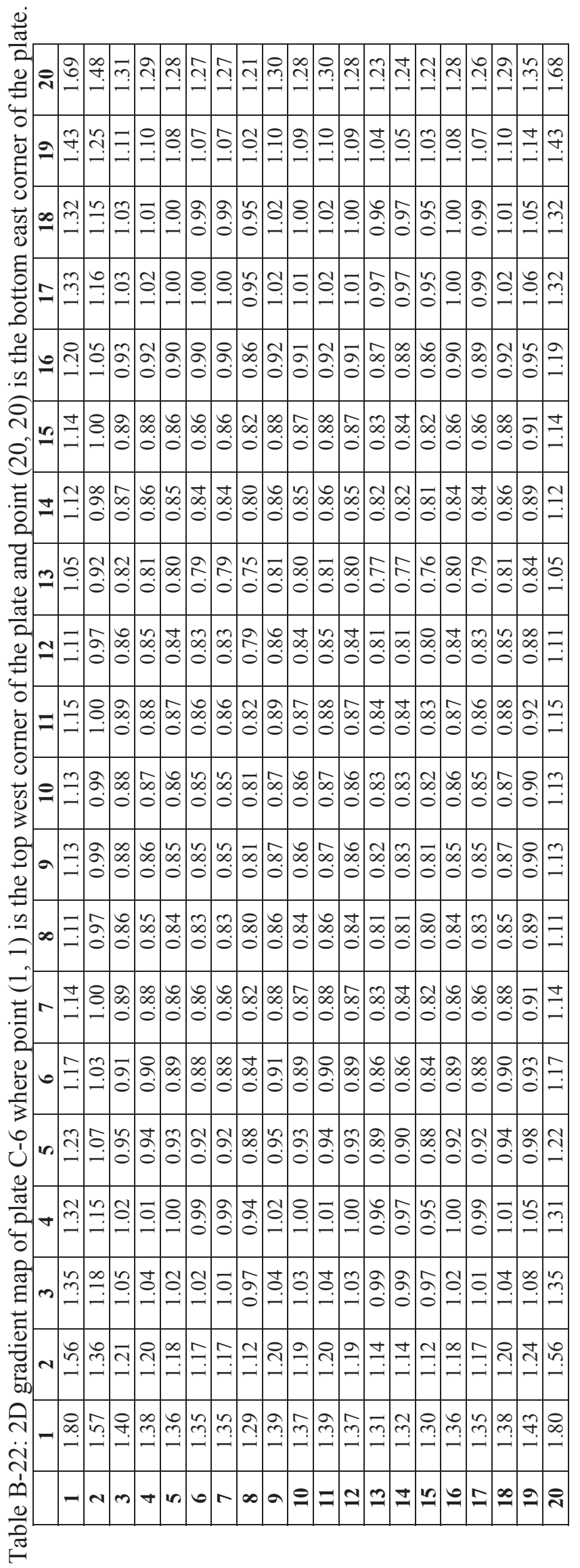

$\frac{0}{\pi}$

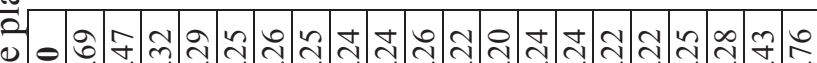

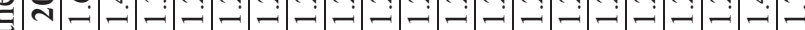

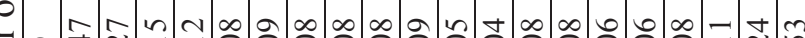

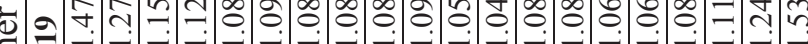
8

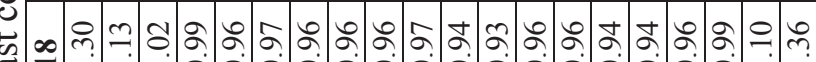

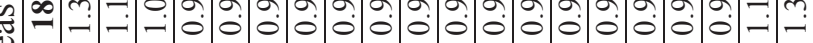

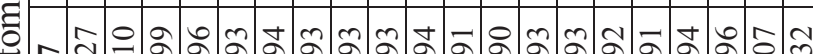
$=1.000 .00$

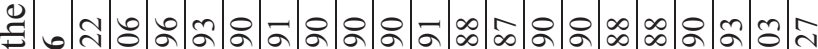
: ป

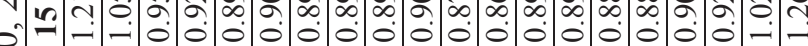

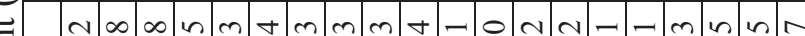

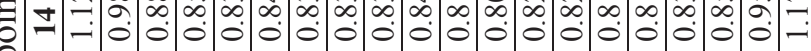

J-s.

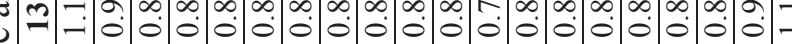

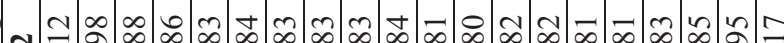

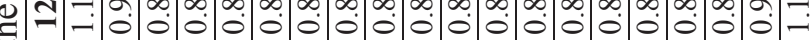

0 r

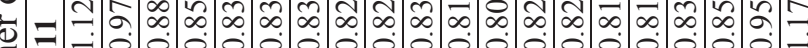

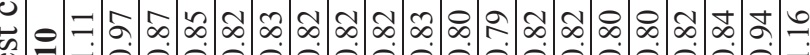

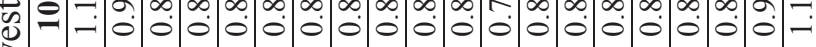

ㅇ

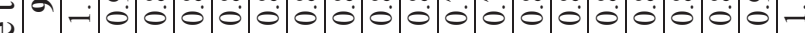

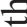

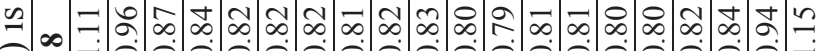

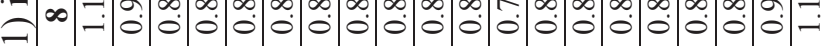

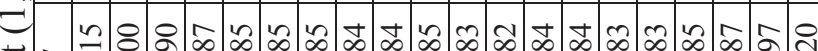

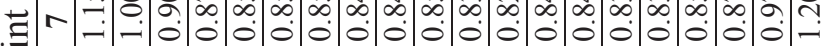

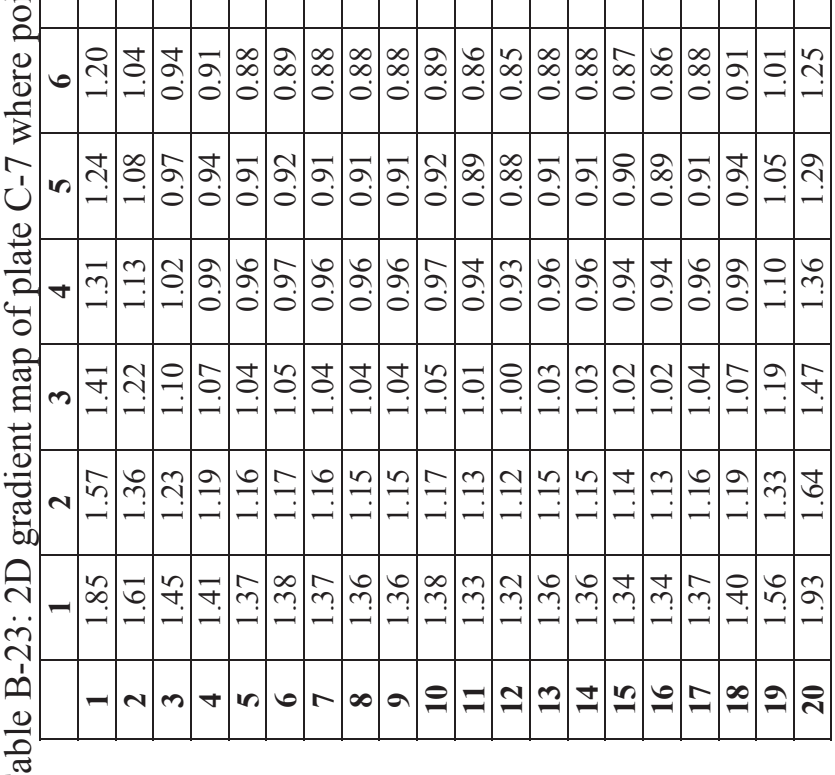
है 


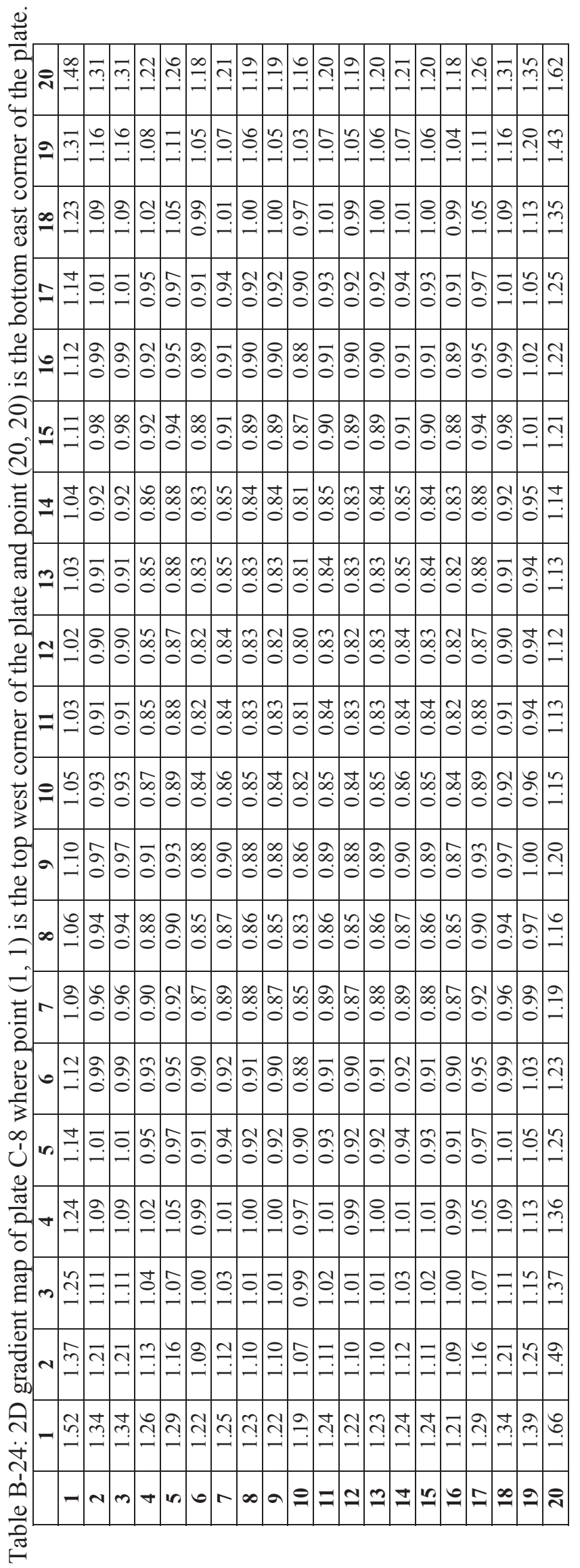

.

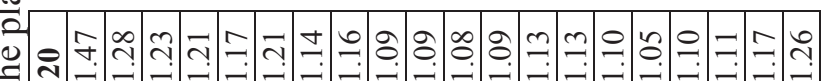

-

ப்

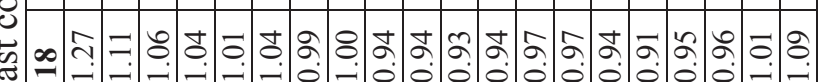
月. = $=0$.

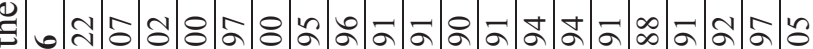

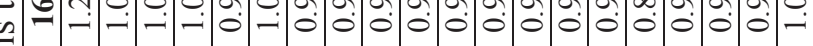

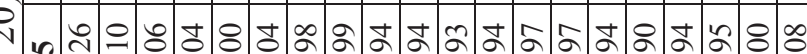

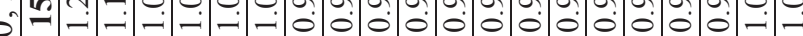
ঊ ন

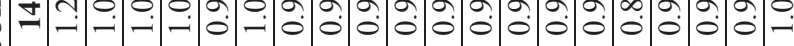

m $=$ t $\infty$ t $\infty$ m

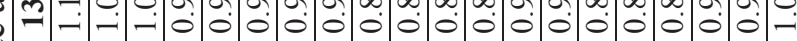

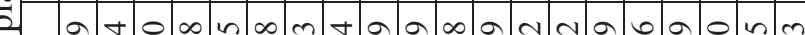
ง

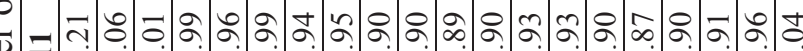

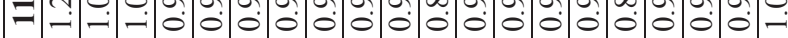

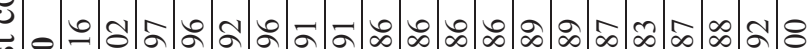

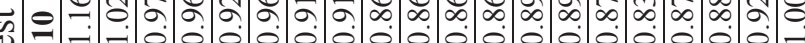
3

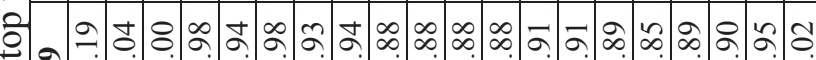

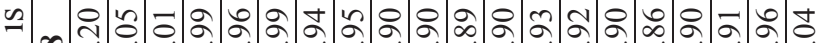

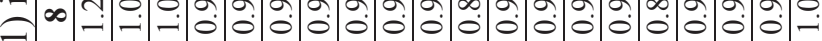

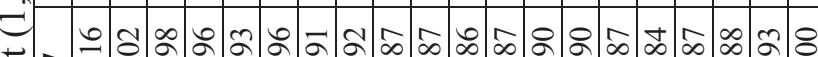

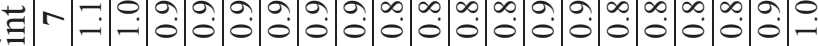

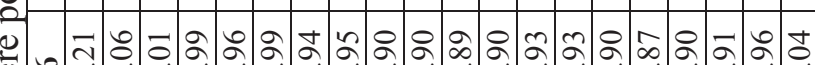
D

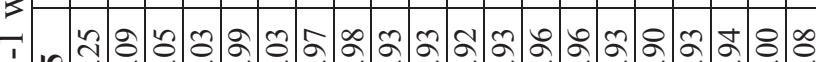
'́ $n$ İ

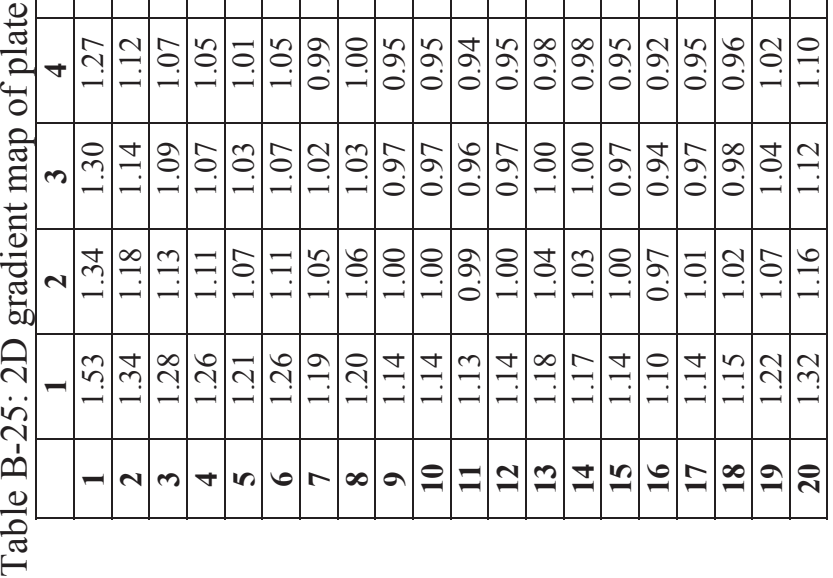




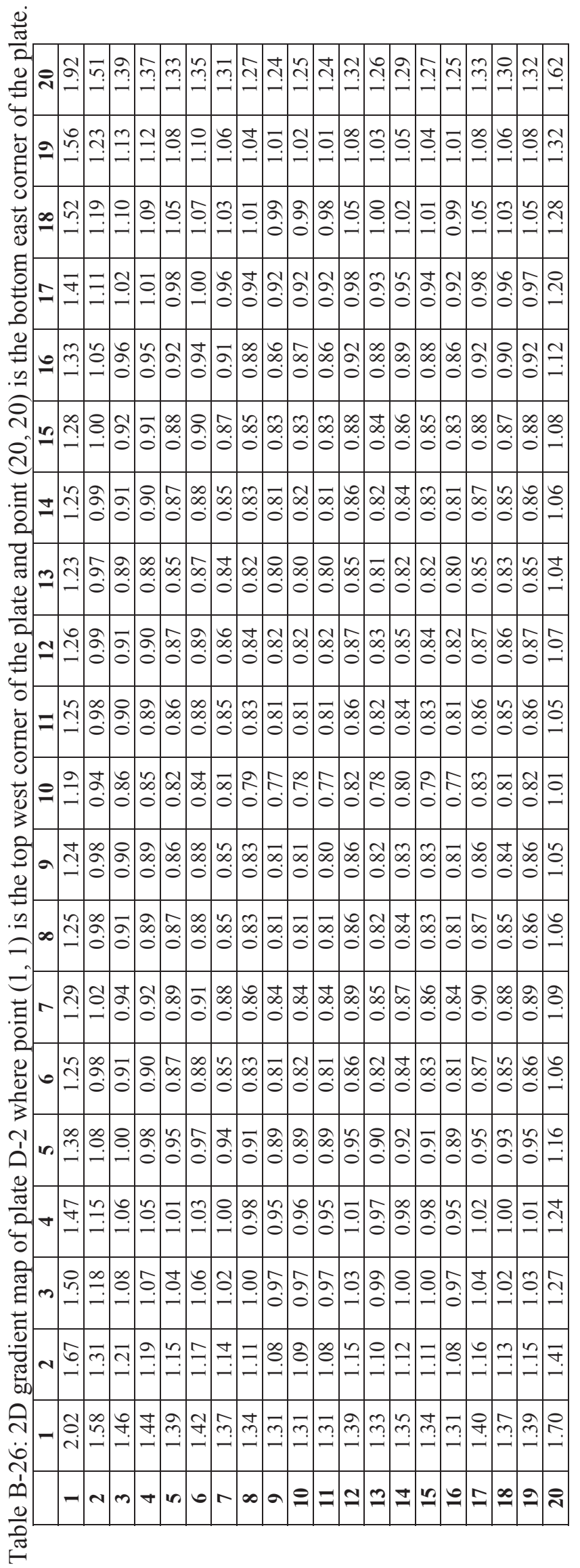

.

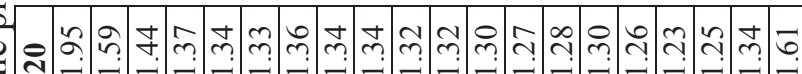

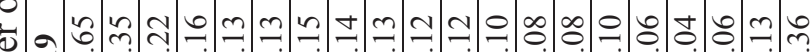
要

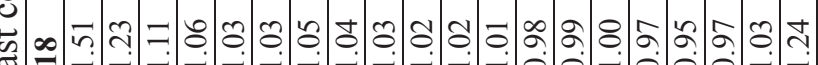
- - - - - -

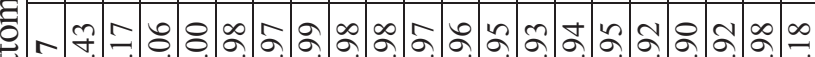

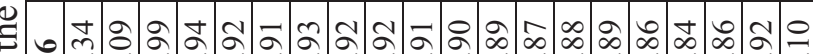

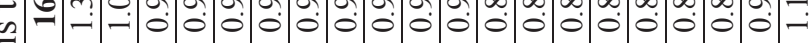
సิ ஊ

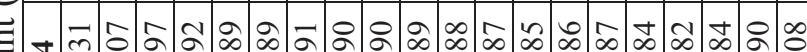

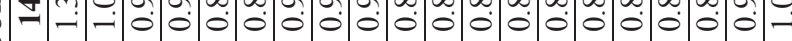

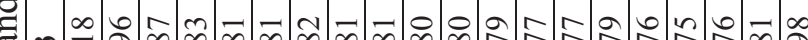

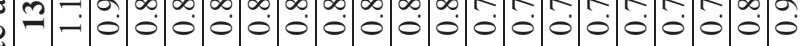
ง

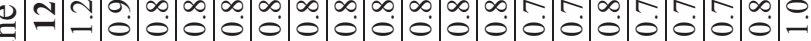

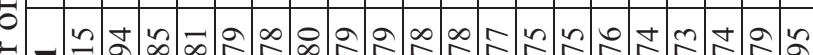

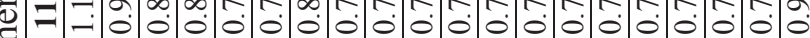

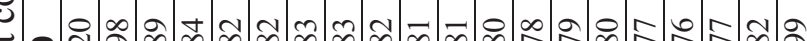
กำ

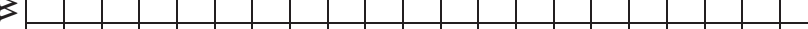
อิ กิ

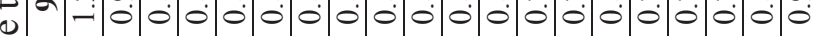

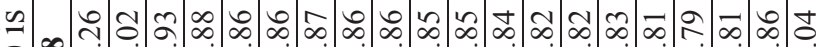

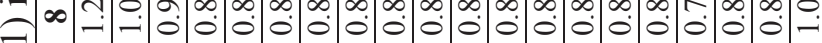

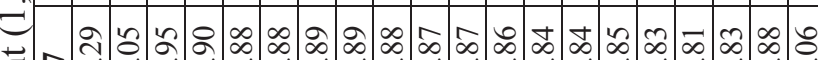

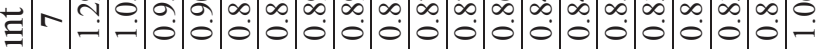

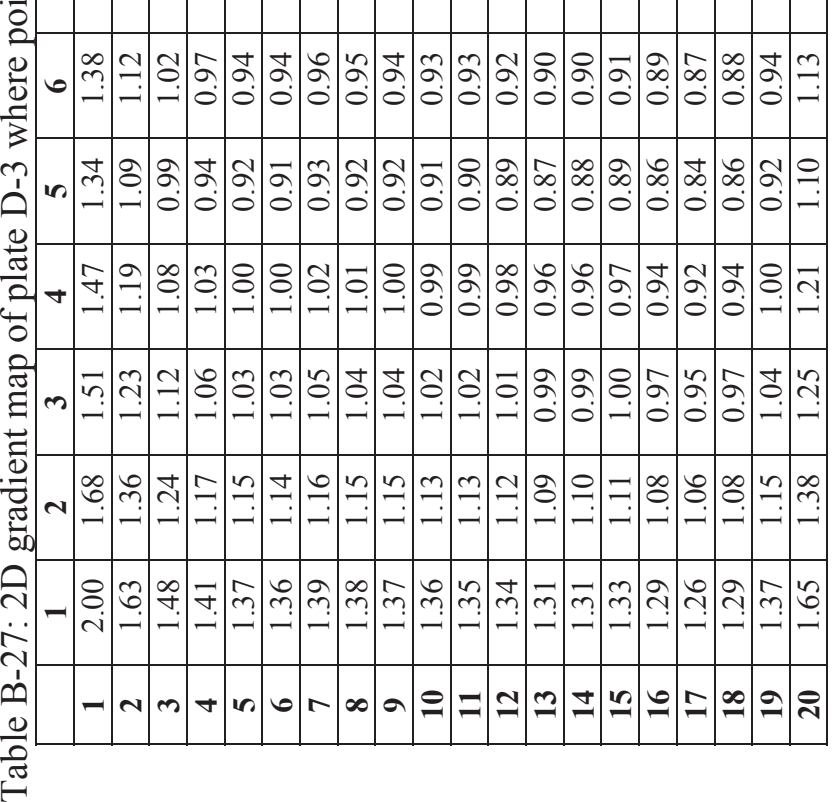




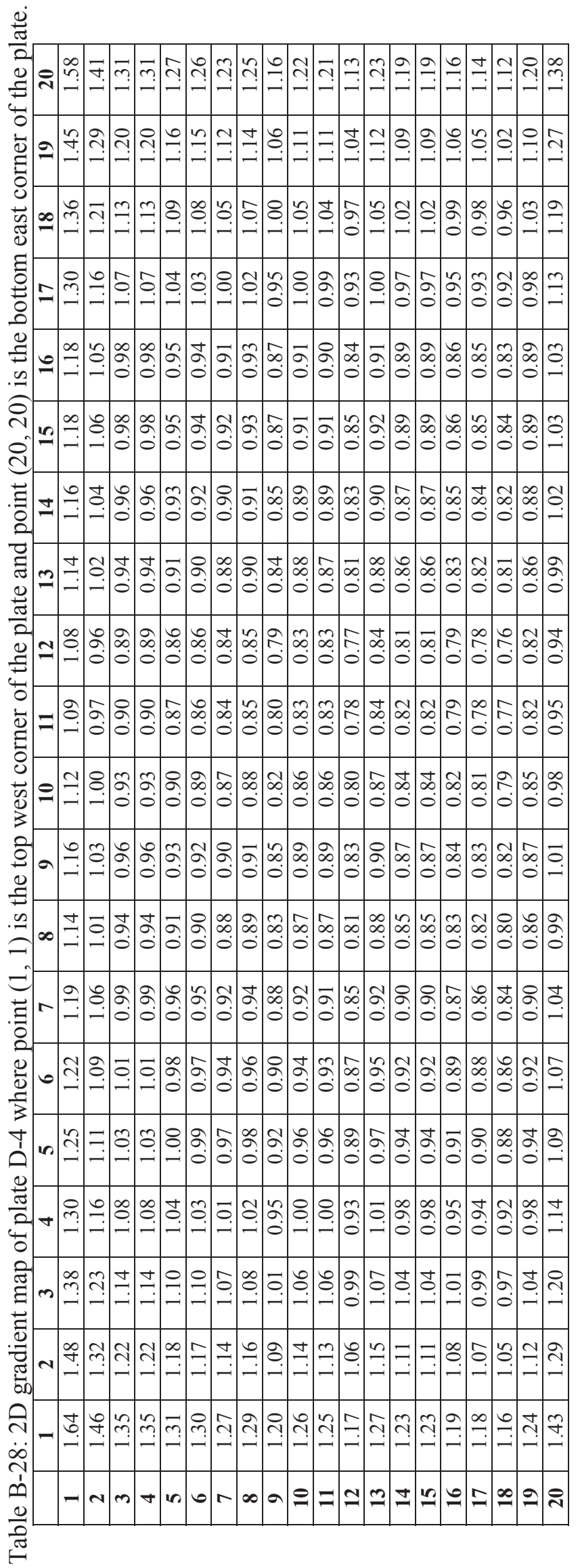

.

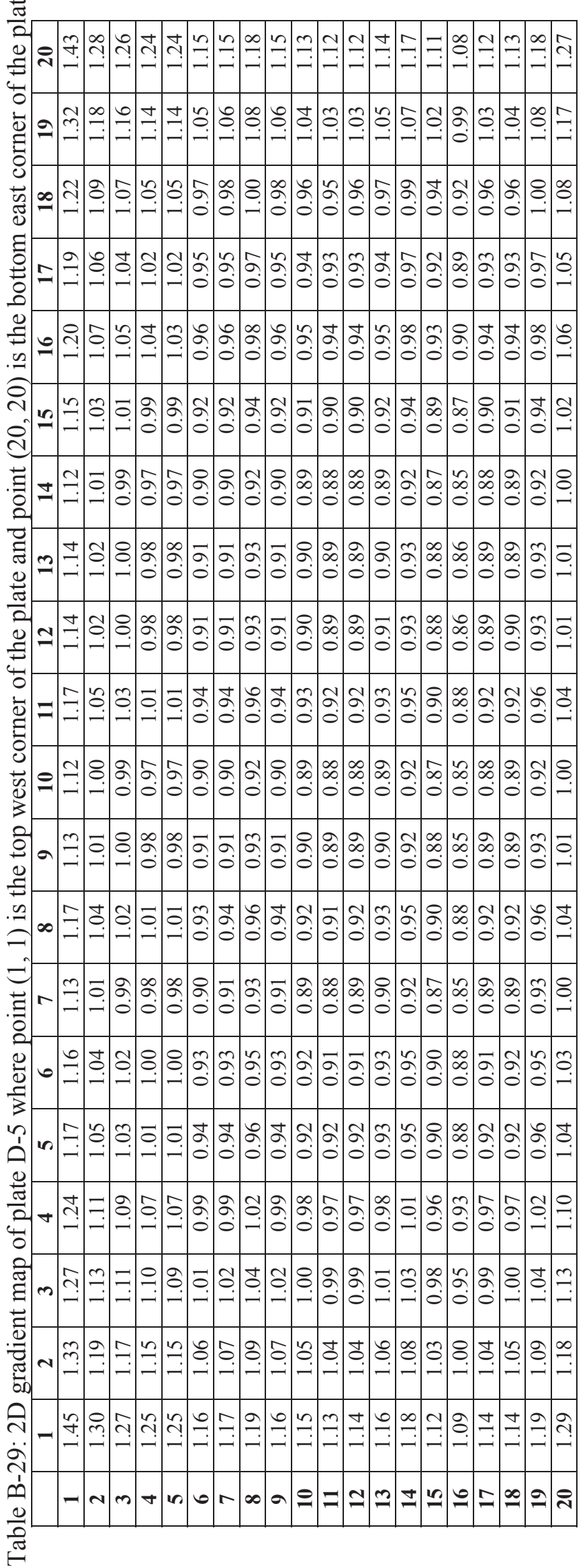




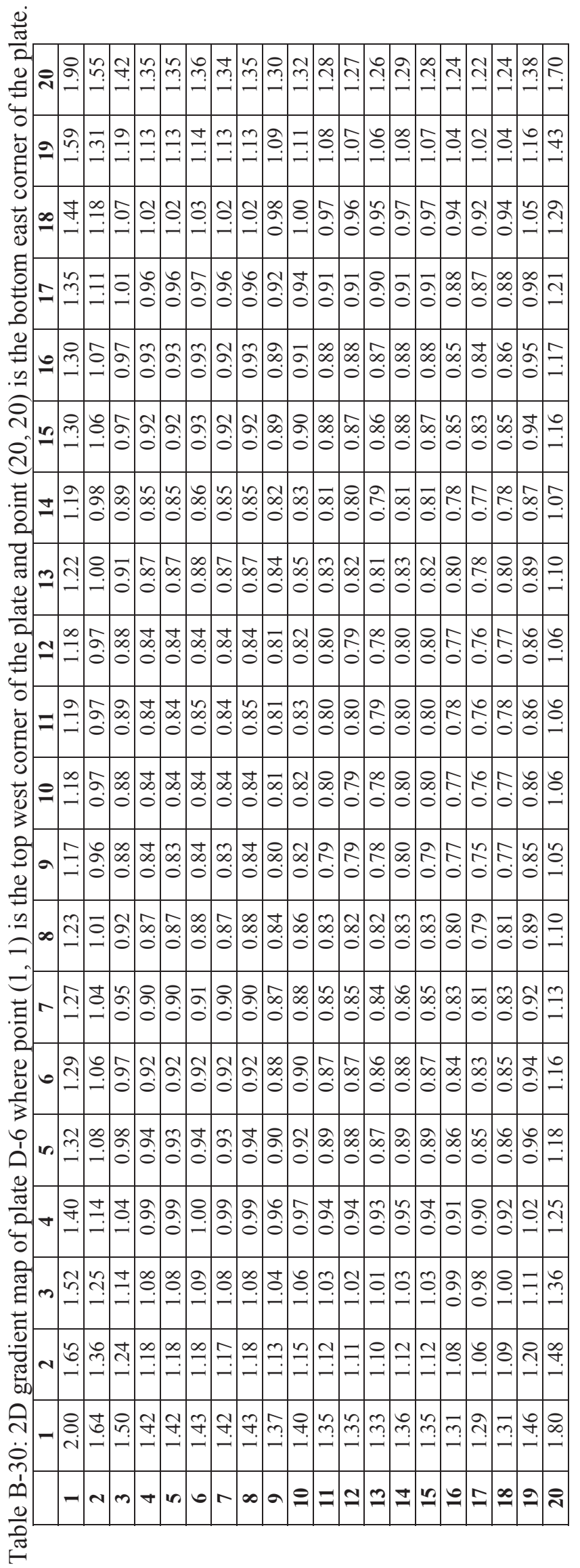

. Dे 

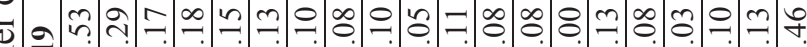
西 要

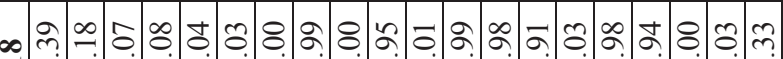
In

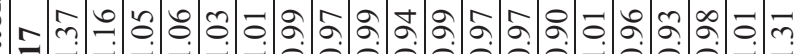

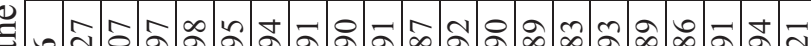

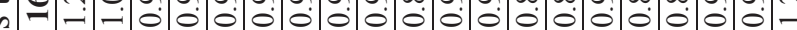
(2)

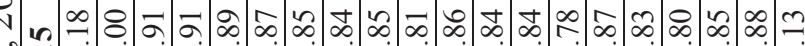

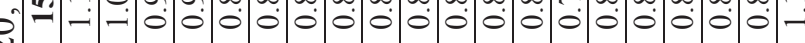

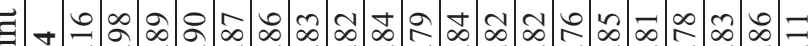

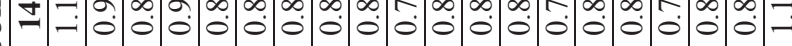

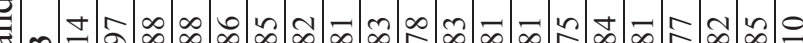

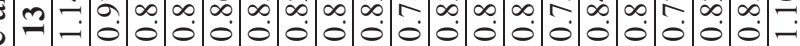

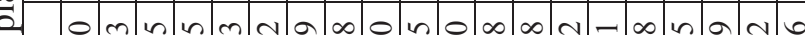

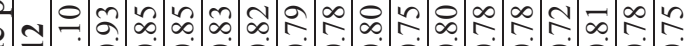

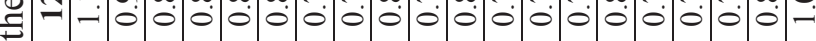

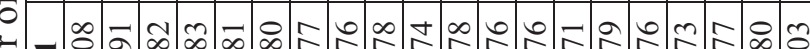
$=\infty \infty \infty \infty \infty$

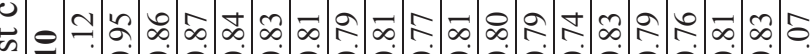

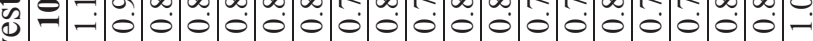

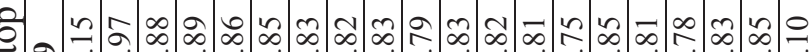
a

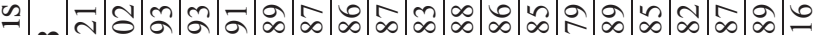

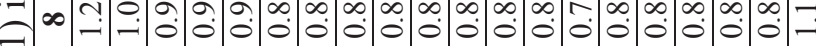
En 0 - 0 n n n

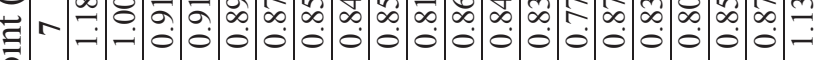

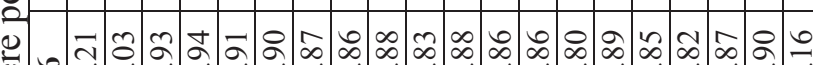

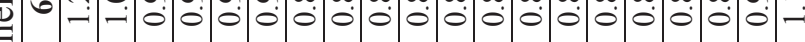
3 च च-

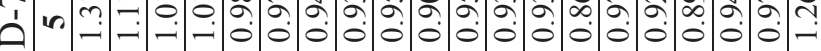
告 n n r m

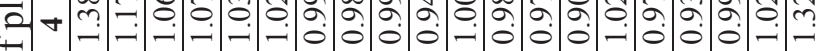
4

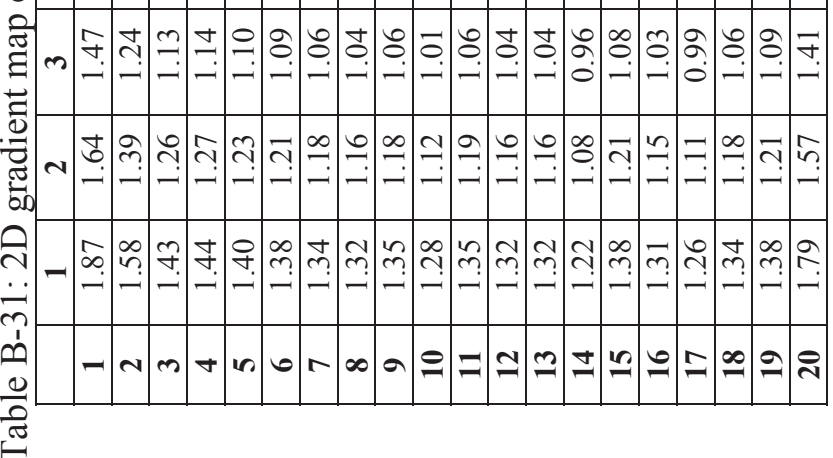




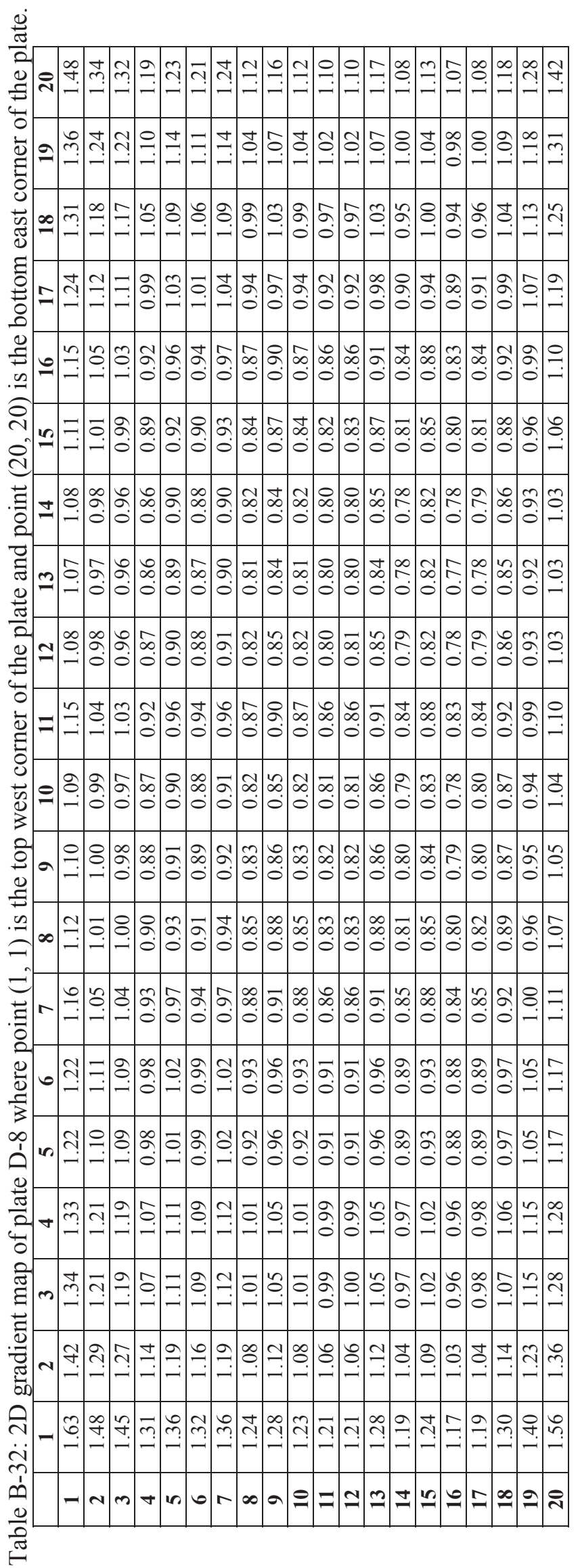

